University of Louisville

ThinkIR: The University of Louisville's Institutional Repository

Electronic Theses and Dissertations

8-2017

\title{
Water-soluble Pd and Pd-alloy nanoparticles as catalysts in biphasic solvent.
}

Shekhar Bhama

University of Louisville

Follow this and additional works at: https://ir.library.louisville.edu/etd

Part of the Materials Chemistry Commons

\section{Recommended Citation}

Bhama, Shekhar, "Water-soluble Pd and Pd-alloy nanoparticles as catalysts in biphasic solvent." (2017). Electronic Theses and Dissertations. Paper 2750.

https://doi.org/10.18297/etd/2750

This Doctoral Dissertation is brought to you for free and open access by ThinkIR: The University of Louisville's Institutional Repository. It has been accepted for inclusion in Electronic Theses and Dissertations by an authorized administrator of ThinkIR: The University of Louisville's Institutional Repository. This title appears here courtesy of the author, who has retained all other copyrights. For more information, please contact thinkir@louisville.edu. 


\title{
WATER-SOLUBLE Pd AND Pd-ALLOY NANOPARTICLES AS CATALYSTS IN BIPHASIC SOLVENT
}

\author{
By
}

Shekhar Bhama

\author{
A Dissertation \\ Submitted to the Faculty of the \\ College of Arts and Sciences of the University of Louisville \\ In Partial Fulfillment of the Requirements \\ For the Degree of
}

Doctor of Philosophy in Chemistry

Department of Chemistry

University of Louisville

Louisville, Kentucky

August 2017 
Copyright 2017 by Shekhar Bhama

All rights reserved 



\section{WATER-SOLUBLE Pd AND Pd-ALLOY NANOPARTICLES AS CATALYSTS IN BIPHASIC SOLVENT}

By

Shekhar Bhama

A Dissertation Approved on

August 4th 2017

By the following Dissertation Committee:

Dissertation Director: Dr. Francis P. Zamborini

Dr. Martin O’Toole

Dr. M. Cecilia Yappert

Dr. Ying Li 


\section{DEDICATION}

This dissertation is dedicated to my wonderful parents

Santosh Kumar Verma and Shashi Verma;

And my loving wife Dr. Preeti Wadhwa 


\section{ACKNOWLEDGEMENTS}

It is a dream come true for me to complete my Ph.D. at the University of Louisville and this would not have been possible without the help and support of countless people over the past five years. First of all, I would like to express my gratitude towards my advisor, Dr. Francis P. Zamborini. His excellent mentoring, leadership quality, attention to minute details, discipline in lab, hard work, intelligence, hold and understanding on the topics have set an example among everybody which I wish to match someday. I gratefully appreciate his encouragement during the time when I was feeling home sick. And with his motivation and believing in me, today, I am here finishing my PhD in chemistry. He has always shown us the right path and aimed us to conquer our dreams.

I want to express my deep appreciation for the faculty members who closely monitored my growth, followed my career and served on my advisory committee: Dr. M. Cecilia Yappert, Dr. Ying Li and Dr. Martin O’Toole. I am also grateful to Dr. William Richmond and Benjamin L. Hutcherson for their help with the gas chromatography experiments; Rodica McCoy, Dr. Tereza Paronyan and Dr. Jacek B. Jasinski from the Conn Center for Renewable Energy Research at the University of Louisville for assistance with TGA, SEM and EDAX analysis. A deep gratitude has 
been given to all the faculty and staff in the Department of Chemistry of the University of Louisville for their help and kindness.

I deeply want to acknowledge all my fellow group members, both past and present: Dr. Francisco Ibañez, Dr. Monica Moreno, Dr. Aiqin Fang, Dr. Nidhi Shah, Dr. Rafael Masitas, Dr. Tulashi Luitel, M.Sc. Irina Kachian, M.Sc. Benjamin L. Hutcherson, Amareshwari Konutham, Badri Mainali, Pom Kharel, Jay Sharma, Stacy Allen, Dhruba Saha and Tirtha Sibakoti who worked with me on the catalysis project. Dr. Monica Moreno mentored and trained me when I first joined the group, showed me how to synthesize and characterize the metal nanoparticles, and since then we have had a wonderful friendship. It has been an honor for me to meet all these nice and talented people who became my family in Louisville. I have learned a lot from them and they have helped make my time in the Ph.D. program more fun and interesting every day.

I also met plenty of people who kept me sane and happy during my graduate studies in the United States. They have always been there for me and I am sure I can always count on them. I especially thank Dr. Rajat Chauhan for giving me all the support through every step of the way and to be my best friend. I am extremely grateful to my loving wife Dr. Preeti Wadhwa who encouraged, understood and loved me at every moment. None of this could have happened without Preeti. This dissertation is dedicated to her.

Despite the geographical distance with my parents, they have always been nearby. It would not have been possible to finish this journey without their unconditional love, motivation and patience. My parents have been my inspiration 
to keep moving forward in my life, and I promise to take care of them for the rest of our future together. I also want to mention my sister Deepti Verma and my nephew Siddharth Rana who make me laugh and forget the stress from school. Finally, I want to thank my grandmother Kamala Wati for her spiritual encouragement, making me aligned to the path of goodness and morality. 
ABSTRACT

\title{
WATER-SOLUBLE Pd AND Pd-ALLOY NANOPARTICLES AS CATALYSTS IN BIPHASIC SOLVENT
}

\author{
Shekhar Bhama
}

August $4^{\text {th }}, 2017$

This dissertation shows the chemical synthesis of $\mathrm{Pd}$ and Pd-alloy nanoparticles (NPs) and their hydrogen and thermal stabilities for applications in the homogeneous and pseudo-homogeneous catalysis of organic reactions. Mainly, this dissertation describes 1) the chemical synthesis of Pd, PdPt, PdAu and Cu-Pd core-shell NPs coated with various organic ligands (thiols, amines and citrate), with different metal compositions in different synthetic environment, 2) the stability of solutions containing various NPs in the presence of hydrogen or varying temperatures, 3) the evaluation of the catalytic activity of Pd, PdPt, PdAu and CuPd NPs for hydrogenation/isomerization of allylic alcohols, and 4) the reactivity of Pd NPs towards the Suzuki-Miyaura reaction. We chemically synthesized water-soluble Pd and Pd-alloy (PdPt and PdAu) NPs stabilized with reduced-L-glutathione (Glu) ligands under both inert and aerobic atmosphere and measured their catalytic 
activity and selectivity towards the hydrogenation/isomerization of allyl alcohol in a biphasic organic solvent/water mixture. We observed that the oxygen synthesized NPs are catalytically more active than the inert ones; while those synthesized under nitrogen have higher recyclability. Interestingly, reactions were $80-90 \%$ selective to the isomer product for all NPs studied. The catalytic activity of various glutathionecoated NPs increased with increasing Au or Pt in the PdAu or PdPt alloy NPs, respectively. Glutathione-coated Pd:Au (50:50) alloy NPs showed best catalyst, exhibiting turnover frequencies of greater than 700 and 100\% conversion for 9 cycles. We synthesized Pd NPs coated with weakly adsorbed citrate ligands under inert nitrogen atmosphere and showed their unique stability in an organic-aqueous biphasic solvent system in the presence of hydrogen gas, high catalytic activity with remarkably high turnover frequencies in the range of 4000 to 8000 , and recyclability with $100 \%$ product conversion towards the hydrogenation/ isomerization of various chain-lengths of $\alpha, \beta$-unsaturated alcohols for up to 5 cycles. We learned that substrate structure plays an important role in the catalytic activity of the NPs, resulting in 1-penten-3-ol being catalytically more active than allyl alcohol and 1-hepten-3-ol. The selectivity showed a similar ratio of 3:2 hydrogenation:isomerization product for all substrates. Interestingly, the synthesized Cu-Pd core-shell NPs showed a selectivity of 95:05 towards the hydrogenated:isomerized product, showing that selectivity can be controlled through metal composition. Finally, we also synthesized $\mathrm{C}_{16 \mathrm{NH}_{2}}$ stabilized Pd NPs and showed their high thermal stability and catalytic activity towards SuzukiMiyaura reaction. Overall, our research has lead to new fundamental insights about 
various topics including the different compositions of metals with Pd, different ligand passivation, importance of synthetic environment (inert or oxygen) and substrate structure, organic solvent used in biphasic system, hydrogen flow rates and their utilization in designing better catalysts with high stability, catalytic activity and recyclability. 
TABLE OF CONTENTS

PAGE

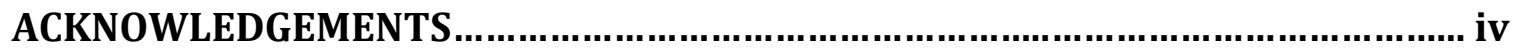

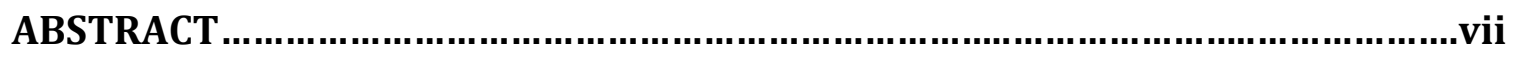

LIST OF TABLES

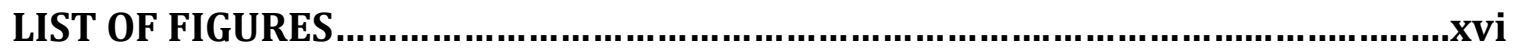

\section{CHAPTER}

I. INTRODUCTION

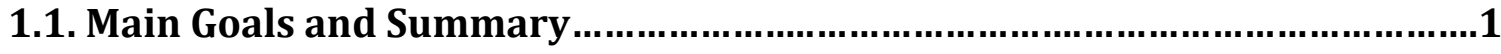

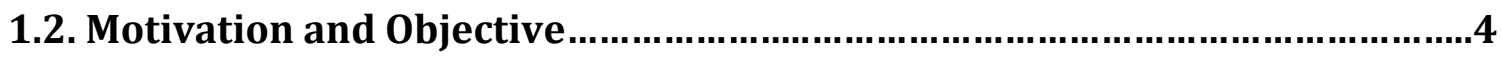

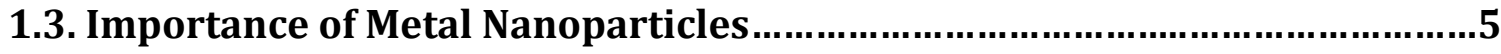

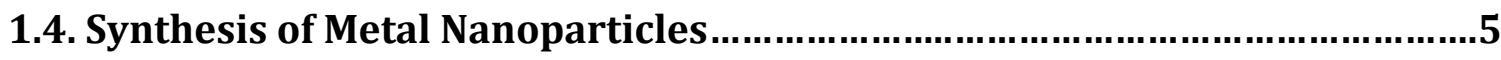

1.4.1. Metal Nanoparticles Coated with Organic Ligands........................................9

1.4.2. Metal Nanoparticles Stabilized Electrostatically ……….............................12

1.4.3. Metal Nanoparticles as Homogenous Catalysts..........................................13

1.5. Catalysis with Chemically Synthesized Metal Nanoparticles.......................16

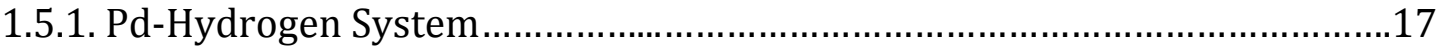


1.6. Catalysis and Metal Nanoparticles.........................................................18

1.6.1. Importance and Reactivity of Allyl Alcohol with Hydrogen..........................23

1.6.2. Mechanism of hydrogenation and isomerization of Allyl Alcohol................26

1.6.3. Importance of Suzuki-Miyaura reaction and its mechanism.......................27

II. EXPERIMENTAL

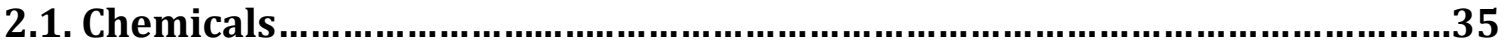

2.2. Synthesis of Pd, PdPt, PdAu and Cu@Pd Nanoparticles (NPs).....................36

2.2.1. Synthesis of Glutathione-Coated Pd, PdPt and PdAu (Inert) NPs..................36

2.2.2. Synthesis of Glutathione-Coated Pd, PdPt and PdAu (Oxy) NPs....................39

2.2.3. Synthesis of Citrate-Coated Pd and CuPd core-shell NPs.............................42

2.2.4. Synthesis of Alkylamine-coated Pd NPs [octylamine $\left(\mathrm{C} \mathrm{NH}_{2}\right)$, dodecylamine $\left(\mathrm{C}_{12} \mathrm{NH}_{2}\right)$, hexadecylamine $\left(\mathrm{C}_{\left.16 \mathrm{NH}_{2}\right)} \mathrm{Pd} \mathrm{NPs}\right] \ldots \ldots \ldots \ldots \ldots \ldots \ldots \ldots \ldots \ldots . . .45$

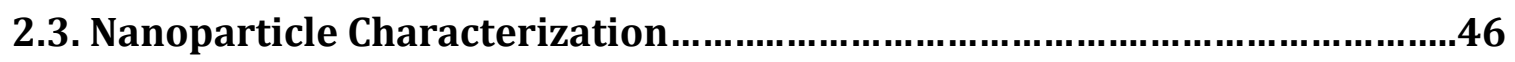

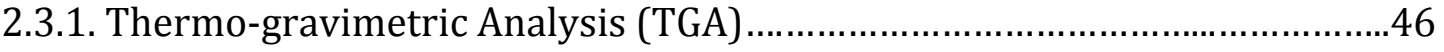

2.3.2. Nuclear Magnetic Resonance Spectroscopy ( ${ }^{1} \mathrm{H}$ NMR) ................................4

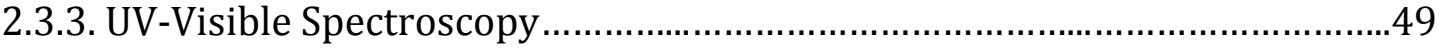

2.3.4. Transmission Electron Microscopy (TEM) ………………………...........50

2.4. Hydrogen Stability of Solution-phase glutathione and citrate-coated Pd,

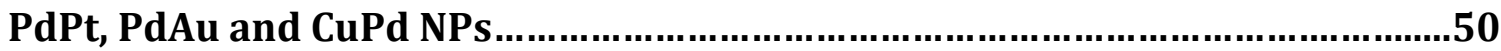

2.5. Catalysis with glutathione-coated and citrate-coated Pd and Pd-alloy

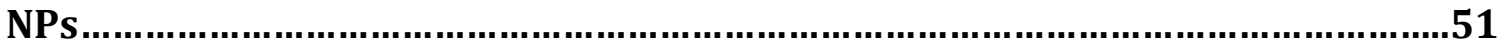

2.5.1. Gas Chromatography-Flame Ionization Detector (GC-FID) .........................53 
2.6. Catalysis studies for the $\mathrm{C}$-C cross coupling Suzuki-Miyaura reaction.......55

III. HYDROGEN AND THERMAL STABILITY OF SOLUTION-PHASE Pd, PdPt AND

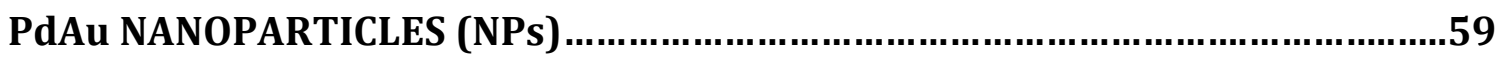

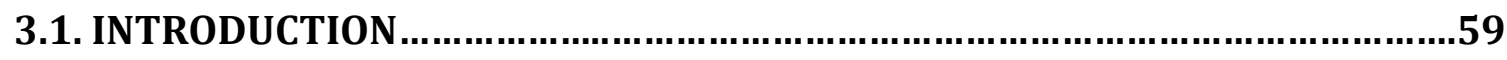

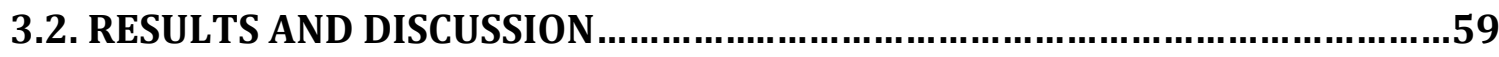

3.2.1. Hydrogen Stability of glutathione-coated Pd NPs....................................60

3.2.2. Hydrogen Stability of citrate-coated Pd NPs.........................................68

3.2.2.1. Stability of Pd-citrate NPs without substrate....................................68

3.2.2.2. Stability of Pd-citrate NPs in presence of substrates........................70

3.2.3. Thermal Stability of Solution-phase alkylamine-coated Pd NPs $\left(\mathrm{C}^{2} \mathrm{NH}_{2}\right.$,

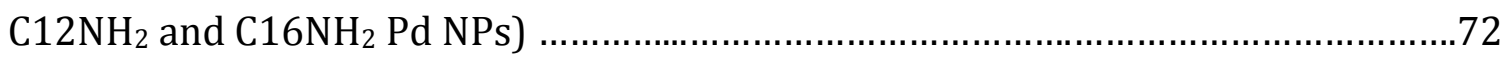

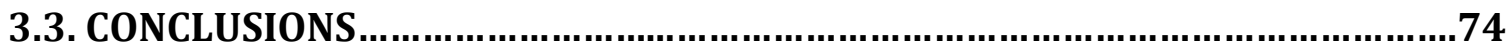

IV. CONTROLLED ACTIVITY AND SELECTIVITY OF GLUTATHIONE-STABILIZED

Pd, PdPt and PdAu NANOPARTICLES (NPs) FOR ISOMERIZATION OF ALLYL

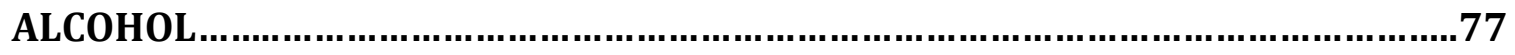

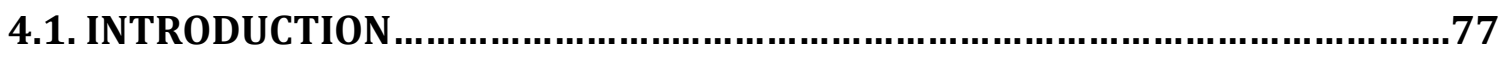

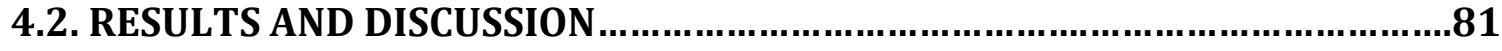

4.2.1. Biphasic catalysis with glutathione-coated NPs ..................................83

4.2.2. Selectivity of glutathione-coated Pd and Pd-alloy NPs..............................89

4.2.3. Role of different flow rates of hydrogen on turnover frequency (TOF) and selectivity of the catalytic reaction. .92 
4.2.4. Role of different organic solvents in the biphasic mixture on turnover frequency (TOF) and selectivity of the catalytic reaction....................................95

4.2.5. Stability comparison of Pd-Glu (Inert) NPs with previously synthesized C6S-Pd NPs

4.2.6. Recyclability of glutathione-coated Pd and Pd-alloy NPs. 97

4.2.7. Recycling stability of glutathione-coated PdAu NPs in various organic solvents 103

4.2.8. Recycling stability comparison between Pd-Glu (Inert) and Pd-Glu (Oxy) NP. 103

4.2.9. Comparison between Inert and Oxygen Pd:Au-Glu NPs 105

4.2.10. Biphasic catalysis for larger chain allylic alcohol substrates 105

4.2.11. Comparison with other Nanoparticles Catalysts

4.3. CONCLUSIONS. 113

V. CATALYSIS STUDIES OF CITRATE-STABILIZED Pd, CuPd CORE-SHELL NANOPARTICLES FOR HYDROGENATION/ISOMERIZATION OF VARIOUS ALLYLIC ALCOHOL 115

5.1. INTRODUCTION 115

5.2. RESULTS AND DISCUSSION. 117

5.2.1. Biphasic hydrogenation/isomerization catalysis with citrate-coated NPs. 117

5.2.2. Turnover Frequencies (TOFs) of Pd catalysts 122

5.2.3. Catalytic activity and selectivity of Pd-citrate NP catalysts 124 
5.2.4. Procedure for recyclability of Pd-citrate NPs

5.2.5. Catalytic comparison between straight chain and branched substrates.. 130

5.2.6. Catalytic comparison between mono-phasic and biphasic solvent

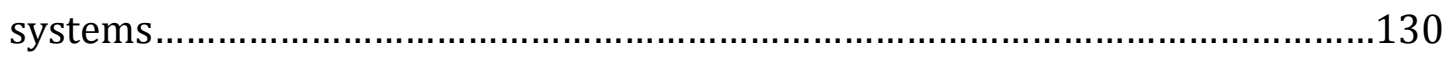

5.2.7. Kinetic studies of catalysis using different allylic alcohols........................132

5.2.8. Electronic effects of CuPd core-shell citrate-stabilized NPs for Improving

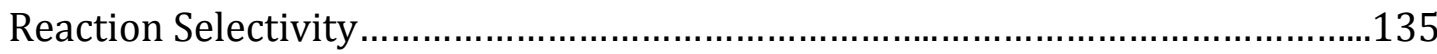

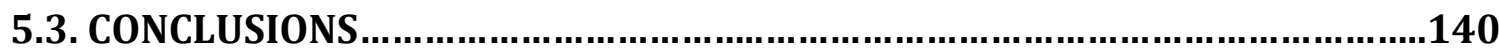

VI. CATALYSIS STUDIES OF ALKYLAMINE-STABILIZED Pd NANOPARTICLES (NPS) FOR SUZUKI-MIYAURA REACTION.....................................................142

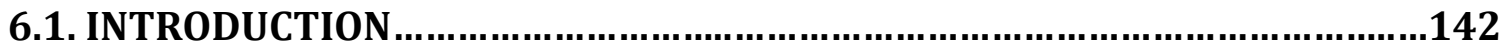

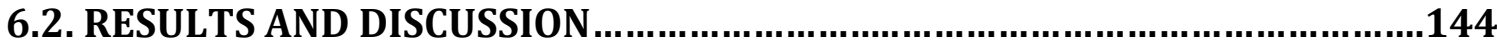

6.2.1. Catalytic Activity of alkylamine-capped Pd NPs.......................................145

6.2.2. Comparison with Other Nanoparticle Catalysts.......................................152

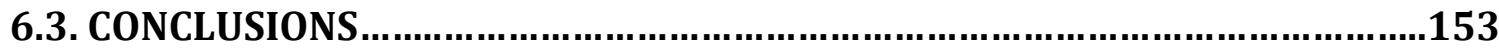

VII. SUMMARY, CONCLUSIONS, AND FUTURE DIRECTIONS............................155

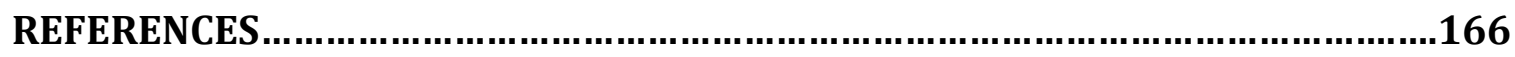

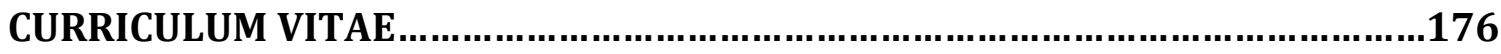




\section{LIST OF TABLES}

\section{TABLE}

2.1. Summary of the synthesis of the metal nanoparticles performed in this dissertation.

4.1. Table showing turnover frequencies (TOF) and recyclability correlation for (75:25) Pd:Au-Glu (Inert/Oxygen) NPs in ethyl acetate, chlorobenzene and dichloromethane organic solvents in biphasic solvent mixture.

5.1. Catalytic comparison (activity, selectivity and recyclability) between different structurally related $\alpha, \beta$-unsaturated alcohols including allyl alcohol, 1-penten-3-ol, 1-hepten-3-ol and 2-methyl-3-buten-2-ol using biphasic solvent system of $2 \mathrm{~mL}$ of as-synthesized aqueous Pd-citrate NPs solution (Pd NPs $=0.15 \mathrm{mg}$ ) and $2 \mathrm{~mL}$ dichloromethane organic solvent at $19.9 \pm 0.7 \mathrm{~mL} / \mathrm{min}$ hydrogen flow rate.

5.2. Catalytic comparison (activity, selectivity and recyclability) between 1penten-3-ol and 2-methyl-3-buten-2-ol having same number of carbon atoms for hydrogenation/isomerization catalysis using Pd-citrate NPs at 19.9 \pm 0.7 $\mathrm{mL} / \mathrm{min}$ hydrogen flow rate using biphasic solvent system of dichloromethane and aqueous phase.

6.1. Table of the different reaction conditions involving the mixing of 4iodoanisole, phenylboronic acid and Pd NP catalyst in organic:aqueous biphasic mixture, where base is dissolved in water and the reaction is either heated or stirred at room temperature for different amounts of time. The reaction condition 9 showed the best results. 


\section{LIST OF FIGURES}

\section{FIGURE}

\section{PAGE}

1.1. Schematic representation of synthesizing metal nanoparticles using physical or chemical methods.

1.2. Schematic representation showing the nucleation, growth and passivation of metal to form stabilized metal NPs and its use in catalysis.

1.3. Schematic representation of a metal nanoparticle surrounded by various hydrophilic organic capping ligands.

1.4. Schematic representation of a reaction involving the synthesis of citratecapped Pd nanoparticles.

1.5. Illustration of $\mathrm{H}_{2}$ adsorption into the $\mathrm{Pd}$ lattice and diffusion causing phase transition from $\alpha$-phase $\mathrm{PdH}_{\mathrm{x}}$ lattice to $\beta$-phase $\mathrm{PdH}_{\mathrm{x}}$.

1.6. Dissociation of hydrogen by breaking $1 \mathrm{~s}-1 \mathrm{~s}$ bonds into the more active atomic hydrogen on the $\mathrm{Pd}$ surface leading to the desirable catalytic properties.

1.7. Schematic illustration of the catalytic redox isomerization reaction using allyl alcohol.

1.8. Proposed mechanisms for the hydrogenation and isomerization as (A) Pd-alkyl mechanism and (B) $\pi$-allyl hydride mechanism for the isomerization reaction of allyl alcohol.

1.9. Mechanism for the Pd catalyzed Suzuki cross-coupling involving three main steps.

1.10. Proposed mechanisms for the Pd NP-catalyzed Suzuki cross-coupling involving both homogeneous and heterogeneous pathways.

2.1. Digital pictures of steps in solution phase synthesis of Pd-Glu (Inert) NPs (A) and Pd-Glu (Oxy) NPs (B). 
2.2. Schematic representation of glutathione-coated Pd, PdPt or PdAu NPs obtained as a product of synthesis.

2.3. Thermogravimetric (TGA) measurements of (A) Pd-Glu (Inert) NPs and (B) Pd-Glu (Oxy) NPs.

2.4. Digital pictures of Pd-Glu (Oxygen) NPs in solid state (A) and dissolved in nanopure water (B).

2.5. Thermogravimetric (TGA) measurement of $\mathrm{C}_{16} \mathrm{NH}_{2} \mathrm{Pd}$ NPs.

2.6. Schematic illustration of hydrogen stability set-up of NPs in solution.

2.7. Biphasic reaction mixture involving aqueous and organic phases (A). And complete set-up of the catalytic reaction involving passing hydrogen gas through the glass pipette into the reaction mixture (B).

2.8. Gas chromatogram of a standard solution containing $10 \mathrm{mM}$ of 2 propen-1-ol (reactant), propanal and 2-propen-1-ol as products and their retention times for both organic phase (Dichloromethane) (A) and aqueous phase (B). Integration was performed using instrument software. Injection volume $=1 \mu \mathrm{L}$, initial temperature $=80^{\circ} \mathrm{C}$, final temperature $=135^{\circ} \mathrm{C}$, ramp $=$ $25^{\circ} \mathrm{C}$, pressure $=14 \mathrm{PSI}$, Detector $=$ FID.

2.9. Gas chromatogram of a model Suzuki reaction containing 4-iodoanisole ( 0.500 gms, 1.0 mole equi.), phenylboronic acid ( 0.338 gms, 1.3 mole equi.), $12 \times \mathrm{x}_{12} \mathrm{NH}_{2} \mathrm{Pd}$ NPs (0.015 gms, 1.5 mole \%), Potassium carbonate (1.181 gms, 4.0 mole equi.), Toluene ( $10 \mathrm{~mL})$, Water ( $1 \mathrm{~mL}, 10 \%$ aqueous solution). Integration was performed using instrument software. Injection volume $=1$ $\mu \mathrm{L}$, initial temperature $=130^{\circ} \mathrm{C}$, final temperature $=200^{\circ} \mathrm{C}$, ramp $=25^{\circ} \mathrm{C}$, pressure $=20 \mathrm{PSI}$, Detector $=$ MS.

3.1. UV-Vis spectra of (A) Pd-Glu (Oxy) nd (B) Pd-Glu (Inert) NPs for exposure time $0-120 \mathrm{~min}$ to $100 \%$ hydrogen bubbling through the aqueous solutions at $19.9 \pm 0.7 \mathrm{~mL} / \mathrm{min}$. The insets show pictures of solutions before exposure to hydrogen at 0 minute and after exposure to hydrogen at 120 minutes.

3.2. UV-Vis spectra of aqueous Pd-Glu (Inert) NP solution against hydrogeninduced aggregation under monophasic conditions in the presence of allyl 
alcohol at $19.9 \pm 0.7 \mathrm{~mL} / \mathrm{min}$ hydrogen flow rate for $0,30,60,90,120$ and 150 minutes.

3.3. UV-Vis spectra of Pd-Glu (Inert) NPs (A) and Pd-Glu (Oxygen) NPs (B) against hydrogen-induced aggregation under biphasic conditions of chlorobenzene and nanopure water in the presence of allyl alcohol at $19.9 \pm 0.7 \mathrm{~mL} / \mathrm{min}$ hydrogen flow rate for $0,5,15,30,60,120,240$ minutes.

3.4. UV-Vis spectra of (75:25) Pd:Au-Glu (inert) NPs under biphasic conditions of chlorobenzene and nanopure water (A) in the absence and (B) in the presence of allyl alcohol at $19.9 \pm 0.7 \mathrm{~mL} / \mathrm{min}$ hydrogen flow rate for 0 , $5,15,30,60,120$ and 240 minutes.

3.5. UV-Vis spectra of (A) freshly prepared Pd-Glu (Inert) NPs and (B) Pd-Glu (Inert) NPs in aqueous solution for 60 days in the presence of hydrogen flow at $19.9 \mathrm{~mL} \pm 0.7 \mathrm{~mL} / \mathrm{min}$ for $0,5,15,30,60$ and 120 minutes.

3.6. UV-Vis spectra of Pd-citrate NPs against hydrogen-induced aggregation in nanopure water in the absence of substrate at $19.9 \pm 0.7 \mathrm{~mL} / \mathrm{min}$ hydrogen flow rate for $0,5,15,30,60$ minutes.

3.7. UV-Vis spectra of Pd-citrate NPs against hydrogen-induced aggregation under biphasic conditions of $2 \mathrm{~mL}$ dichloromethane and $2 \mathrm{~mL}$ nanopure water at $19.9 \pm 0.7 \mathrm{~mL} / \mathrm{min}$ hydrogen flow rate for $0,5,15,30,60$ minutes in the presence of $0.74 \times 10^{-3}$ moles of (A) allyl alcohol, (B) 1-penten-3-ol, (C) 1hepten-3-ol, and (D) 2-methyl-3-buten-2-ol.

3.8. UV-Vis plots for different temperatures (A) ranging from 25 to $95^{\circ} \mathrm{C}$ for $\mathrm{C}_{16} \mathrm{NH}_{2} \mathrm{Pd}, \mathrm{C}_{12} \mathrm{NH}_{2} \mathrm{Pd} \& \mathrm{C}_{2} \mathrm{NH}_{2} \mathrm{Pd} \mathrm{NPs}$ (B) ranging from 30 to $110^{\circ} \mathrm{C}$ with $\mathrm{C}_{16 \mathrm{NH}_{2}} \mathrm{Pd} \mathrm{NPs}$ aqueous solution and (C) ranging from $40^{\circ} \mathrm{C}-70^{\circ} \mathrm{C}$ with $\mathrm{Pd}$ NPs with all reactants heated for 30 minutes at each temperature.

4.1. The conversion of allyl alcohol to saturated alcohol and aldehyde in the presence of hydrogen and Pd or Pd alloy NP catalyst.

4.2. Schematic illustration of a biphasic catalytic reaction using $2 \mathrm{~mL}$ of organic solvent (ethyl acetate, dichloromethane or chlorobenzene) and $2 \mathrm{~mL}$ of nanopure water and glutathione-coated Pd NPs as a catalyst for the conversion of allyl alcohol as a substrate. 
4.3. Gas chromatograms of organic layer (A) and aqueous layer (B) of a biphasic solvent mixture showing the progress of the catalytic reaction of allyl alcohol before and after exposure to $100 \%$ hydrogen $(19.9 \pm 0.7 \mathrm{~mL} / \mathrm{min})$ at different reaction times of $0,5,10,15,20$ and 25 minutes. The reaction was catalyzed by $\sim 6.0 \mathrm{mg}$ of Pd-glu (Oxy) NPs in biphasic solvent mixture of $2 \mathrm{~mL}$ water and $2 \mathrm{~mL}$ chlorobenzene under atmospheric pressure and at room temperature. Integration of peaks for analysis was performed using instrument software. Injection volume $=1 \mu \mathrm{L}$, initial temperature $=80^{\circ} \mathrm{C}$, final temperature $=135^{\circ} \mathrm{C}, \mathrm{ramp}=25^{\circ} \mathrm{C} / \mathrm{min}$, pressure $=12-14 \mathrm{PSI}$, Detector $=$ FID. 85

4.4. Plot of the \%hydrogenation, \%isomerization and \%total conversion of allyl alcohol versus reaction time using Pd-Glu (Oxy) NP catalysts in biphasic solvent mixture of chlorobenzene and nanopure water.

4.5. Plot of turnover frequencies (TOF, in moles product/moles $\mathrm{Pd} /$ hour) towards hydrogenated and isomerized products for all synthesized Pd and Pd-alloy NPs under inert/oxygen conditions at $19.9 \pm 0.7 \mathrm{~mL} / \mathrm{min}$ hydrogen flow rate using chlorobenzene organic solvent with aqueous phase.

4.6. Box plot showing the reactivity towards the hydrogenated and isomerized products after one complete cycle of reaction between allyl alcohol and $100 \%$ hydrogen at $19.9 \pm 0.7 \mathrm{~mL} / \mathrm{min}$ flow-rate using chlorobenzene and water biphasic solvent system for all synthesized glutathione-coated NPs [Pd-, Pd/Pt- and Pd/Au-glu (Inert/Oxygen) NPs]. All NPs are highly selective towards the isomer product.

4.7. Effects of three different flow rates of $100 \%$ hydrogen $(8.0 \pm 0.2,19.9 \pm 0.7$ and $39.9 \pm 0.3 \mathrm{~mL} / \mathrm{min}$ ) on (A) turnover frequencies (TOF) and (B) selectivity of the overall catalytic reaction using Pd-Glu (Inert/Oxygen) NPs.

4.8. Plot showing the total percent completion of the catalytic reaction vs. time (minutes) for Pd-Glu (Inert) NPs at $8.0 \pm 0.3,19.9 \pm 0.7$ and $39.9 \pm 0.7$ $\mathrm{mL} / \mathrm{min}$ hydrogen flow rate using chlorobenzene and aqueous phase. 
4.9. Effects of three different organic solvents (DCM, EtOAc, Clben) on (A) turnover frequencies (TOF) and (B) selectivity of the overall catalytic reaction using (75:25) Pd:Au-Glu (Inert/Oxygen) NPs.

4.10. Plots showing comparison of hydrogenated-, isomerized- and overallTOFs for (A) Pd-Glu (Inert) NPs and (B) C6S-Pd NPs ${ }^{75}$ at 3 different flow rates of $100 \%$ hydrogen $(8.0 \pm 0.3,19.9 \pm 0.7$ and $39.9 \pm 0.3 \mathrm{~mL} / \mathrm{min})$.

4.11. GC-FID chromatograms of aqueous and organic phases for the recyclability of (75:25) Pd:Au-Glu (Inert) NPs showing 100\% completion of the catalytic reaction for at least 9 times (checked 12 times) with dichloromethane at $8.0 \pm 0.2 \mathrm{~mL} / \mathrm{min}$ hydrogen flow rate.

4.12. Plot showing the recyclability of various $\mathrm{Pd} \& \mathrm{Pd}$-alloy nanoparticles using ethyl acetate (EtOAc), chlorobenzene (Clben) and dichloromethane (DCM) at different hydrogen flow rates with $100 \%$ completion of the reaction.

4.14. Recycling Stability of Pd-Glu (Inert) (above) and Pd-Glu (Oxyen) NPs (below) for 6 cycles of hydrogenation/isomerization of allyl alcohol in the presence of chlorobenzene/nanopure water biphasic solvent system by UVVis spectroscopy.

4.15. Gas chromatograms of organic layer (A) of a biphasic solvent mixture of $2 \mathrm{~mL}$ nanopure water and $2 \mathrm{~mL}$ ethyl acetate showing progress of the catalytic reaction of 1-penten-3-ol before and after exposure to $100 \%$ hydrogen $(19.9 \pm 0.7 \mathrm{~mL} / \mathrm{min})$ at different reaction times from $0,5,10$ and 15 minutes using $~ 6.0 \mathrm{mg}$ of (75:25) Pd:Au-Glu (Oxy) NPs. Frame (B) shows the recyclability of (75:25) Pd:Au-Glu (Oxy) NPs for 1-penten-3-ol up to 5 cycles showing $\%$ hydrogenation, $\%$ isomerization and $\%$ total yield.

4.16. Gas chromatograms of organic layer (A) of a biphasic solvent mixture of $2 \mathrm{~mL}$ nanopure water and $2 \mathrm{~mL}$ chlorobenzene showing the progress of the catalytic reaction of 3-buten-2-ol before and after exposure to $100 \%$ hydrogen $(19.9 \pm 0.7 \mathrm{~mL} / \mathrm{min})$ at different reaction times from $0,5,10$ and 15 minutes using $\sim 6.0 \mathrm{mg}$ of (75:25) Pd:Au-Glu (Oxy) NPs. Frame (B) shows the 
recyclability of (75:25) Pd:Au-Glu (Oxy) NPs for 3-buten-2-ol up to 5 cycles showing $\%$ hydrogenation, $\%$ isomerization and $\%$ total yield.

4.17. Plot showing catalytic activity comparison of 2 years old synthesized (75:25) Pd:Au-Glu (Inert) NPs versus newly synthesized (75:25) Pd:Au-Glu (Inert) NPs for hydrogenation/isomerization of allyl alcohol in terms of hydrogenation \%, isomerization $\%$ and overall conversion $\%$ at $19.9 \pm 0.7$ $\mathrm{mL} / \mathrm{min}$ hydrogen flow-rate using ethyl acetate-aqueous biphasic solvent mixture.

5.1. Biphasic catalytic reactions showing the conversion of allyl alcohol, 1penten-3-ol, 1-hepten-3-ol and 2-methyl-3-buten-2-ol to saturated alcohol (hydrogenated product) and/or aldehyde (isomerized product) in the presence of hydrogen and catalyst.

5.2. Schematic illustration of a biphasic catalytic reaction using $2 \mathrm{~mL}$ of dichloromethane (DCM) and $2 \mathrm{~mL}$ of nanopure water as solvent citratecoated Pd NPs as the catalyst and various chain-lengths of allyl alcohols (2propen-1-ol, 1-penten-3-ol, 1-hepten-3-ol or 2-methyl-3-buten-2-ol) as substrate.

5.3. Stability of Pd-citrate NPs in the presence of $0.74 \times 10^{-3}$ moles of allyl alcohol at $19.9 \pm 0.7 \mathrm{~mL} / \mathrm{min}$ hydrogen flow rate in the biphasic solvent mixture of nanopure water and (A) ethyl acetate or chlorobenzene (not shown) after 5 minutes and (B) dichloromethane after 40 minutes.

5.4. Example of biphasic catalysis for $0.74 \times 10^{-3}$ moles of allyl alcohol using 2 $\mathrm{mL}$ of aqueous Pd-citrate NPs solution and $2 \mathrm{~mL}$ dichloromethane solvent using $19.9 \pm 0.7 \mathrm{~mL} / \mathrm{min}_{2}$ flow rate for 40 minutes. GC-FID data for organic layer (A) and aqueous layer (B) a 0, 5, 10, 15, 20, 30 and 40 minutes reaction times with retention times for both reactant and products shown.

5.5. Example of calculating TOF using plot of \% hydrogenation and $\%$ isomerization of $0.74 \times 10^{-3}$ moles of 1 -hepten-3-ol versus reaction time over $2 \mathrm{~mL}$ aqueous Pd-citrate NPs solution and $2 \mathrm{~mL}$ dichloromethane at $19.9 \pm 0.7$ $\mathrm{mL} / \mathrm{min}$ flow rate of hydrogen and used for determining slope to calculate TOF when $>60 \%$ of 1 -hepten-3-ol was reacted. 
5.6. UV-Vis spectra showing lower stability of Pd-citrate NPs by migrating to the organic phase during the reaction with $0.74 \times 10^{-3}$ moles of 1 -hepten-3-ol at $19.9 \pm 0.7 \mathrm{~mL} / \mathrm{min}$ hydrogen flow rate under biphasic solvent system of 2 $\mathrm{mL}$ of as-synthesized aqueous Pd-citrate NPs solution (Pd NPs $=0.15 \mathrm{mg}$ ) and $2 \mathrm{~mL}$ dichloromethane organic solvent.

126

5.7. GC-FID spectra of aqueous and organic phases for the recyclability of Pdcitrate NPs showing $100 \%$ completion of the catalytic reactions with $0.74 \mathrm{x}$ $10^{-3}$ moles of substrate for at least (A) 3 times for allyl alcohol, (B) 5 times for 1-penten-3-ol and (C) 2 times for 1-hepten-3-ol with dichloromethane at $19.9 \pm 0.7 \mathrm{~mL} / \mathrm{min}$ hydrogen flow rate.

5.8. GC-FID spectra of mono-phasic hydrogenation/isomerization reaction using $0.74 \times 10^{-3}$ moles of 1 -penten-3-ol at $19.9 \pm 0.7 \mathrm{~mL} / \mathrm{min}$ hydrogen flow rate in the presence of $2 \mathrm{~mL}$ nanopure water without using dichloromethane organic solvent.

5.9. Plot showing the total percentage completion of the catalytic reaction vs. time (in minutes) for biphasic solvent mixture containing $2 \mathrm{~mL}$ of assynthesized aqueous Pd-citrate NPs solution and $2 \mathrm{~mL}$ dichloromethane and $19.9 \pm 0.7 \mathrm{~mL} / \mathrm{min}$ hydrogen flow rate in the presence of $0.74 \times 10^{-3}$ moles of (a) allyl alcohol, (b) 1-penten-3-ol and (c) 1-hepten-3-ol.

5.10. GC-FID spectra of $(A)$ aqueous and (B) organic phases for the catalytic activity of $0.15 \mathrm{mg}$ of $\mathrm{PdCl}_{4}^{2-}$ salt in aqueous solution for $0.74 \times 10^{-3}$ moles of allyl alcohol at $19.9 \pm 0.7 \mathrm{~mL} / \mathrm{min}$ of hydrogen flow rate in biphasic $2 \mathrm{~mL}$ dichloromethane and $2 \mathrm{~mL}$ aqueous solvent mixture.

5.11. GC-FID spectra of (A) organic and (B) aqueous phases for the catalytic activity in biphasic solvent mixture containing $2 \mathrm{~mL}$ of as-synthesized aqueous citrate-capped CuPd NPs solution and $2 \mathrm{~mL}$ dichloromethane with $0.74 \times 10^{-3}$ moles of 1 -penten-3-ol at $19.9 \pm 0.7 \mathrm{~mL} / \mathrm{min}$ of hydrogen flow rate for 60 minutes.

5.12. Plot showing the selectivity of the citrate-capped CuPd NPs for the catalytic reaction of $0.74 \times 10^{-3}$ moles of 1 -penten-3-ol at $19.9 \pm 0.7 \mathrm{~mL} / \mathrm{min}$ of hydrogen flow rate for 60 minutes. 
6.1. Reaction scheme showing the utilization of aryl halide and phenylboronic acid in the presence of a catalyst, base and solvent under heating to form unsymmetrical biaryl product for C-C cross coupling SuzukiMiyaura reaction.

6.2. Reaction scheme showing the best possible reaction conditions involving the mixing of 4-iodoanisole (1.0 mole equivalent), phenylboronic acid (1.3 mole equivalent) and Pd NPs catalyst (1.5 mole \%) in toluene:water (12 mL:1 $\mathrm{mL}$ ) biphasic mixture, where potassium carbonate (4.0 mole equivalent) base was dissolved in water, and the reaction mixture heated at $70^{\circ} \mathrm{C}$.

6.3. Product confirmation for the model Suzuki-Miyaura reaction involving 4-iodoanisole ( $0.500 \mathrm{gms}, 1.0$ mole equi.), phenylboronic acid ( $0.338 \mathrm{gms}, 1.3$ mole equi.), $\mathrm{C}_{16 \mathrm{NH}_{2}} \mathrm{Pd}$ NPs (0.015 gms, 1.5 mole \%), $\mathrm{K}_{2} \mathrm{CO}_{3}$ as base (1.181 gms, 4.0 mole equi.) and toluene:water (12 mL:1 mL) biphasic solvent mixture at $70^{\circ} \mathrm{C}$ showing (A) TLC and (B) Aggregation of $\mathrm{C}^{16 \mathrm{NH}_{2}} \mathrm{Pd} \mathrm{NPs}$ after completion of the reaction and (C) Gas chromatography of the final reaction mixture. Products were confirmed using mass spectroscopy.

6.4. UV-vis spectra of the catalyst dissolved in the reaction mixture containing various ratios of catalyst: reactant mixture ranging from $1 / 10,000,1 / 1000,1 / 100$ and $1 / 10$ for different amounts of time ranging from $0,15,30,45$ and 60 minutes. 


\section{CHAPTER I}

\section{INTRODUCTION}

\subsection{Main Goals and Summary}

The main goal of this research was to synthesize highly stable and reactive ligand-stabilized Pd and Pd-containing alloy (PdPt, $\mathrm{PdAu}, \mathrm{CuPd}$ ) nanoparticles (NPs) for potential use in catalysis applications. This dissertation is divided into seven main parts. Chapter I contain background information about all the topics related to this research, including the importance, synthesis and main applications of metal NPs. Chapter II includes the chemicals, instrumentations that were used and the experimental procedures in this research. It describes the chemical synthesis of $\mathrm{Pd}$, PdPt, PdAu and CuPd NPs stabilized with various organic ligands. The composition of all of the NPs is also determined by using various materials characterization techniques. In Chapter III, we report on the study of the stability of solutions containing metal NPs in the presence of hydrogen. We demonstrated that the metal and organic composition of the NPs play a fundamental role in the stability against $\mathrm{H}_{2}$-induced aggregation in solution. Chapter III also describes studies on the thermal stabilities of alkylamine-stabilized Pd NPs for use in Suzuki-Miyaura reactions. Chapter IV and V include an evaluation of the catalytic properties of Pd, PdPt and 
PdAu and $\mathrm{CuPd}$ in the pseudo-homogenous catalysis of organic reactions under biphasic solvent system in the presence of hydrogen. The results in Chapter IV show that all of the Pd, PdPt and PdAu NPs coated with glutathione ligands are highly catalytically active and can be used to catalyze the hydrogenation and isomerization of allyl alcohol with hydrogen to selectively produce the isomer product propanal. We show that the catalytic activity of the NPs can be controlled by its synthesis under inert or oxygen condition and by changing various alloy compositions. Also, the flow rate of hydrogen and use of different types of organic solvents can alter the overall turnover frequencies (TOFs). These NPs can be reused for at least 9 cycles, giving $100 \%$ product yields with high selectivity for the isomerized product. They can be used for up to 12 cycles with a slight decrease in catalytic activity.

Following this work, we continued with the use of Pd:Au-Glu NPs (Glu=Glutathione) as a pseudo-homogeneous catalyst for the hydrogenation/ isomerization of branched allyl alcohols that differ only slightly in their chemical structure in a biphasic solvent mixture. In general, we observed that the larger substrates with longer hydrocarbon chains have lower reaction rates, likely due to restricted diffusion and access to the $\mathrm{Pd}$ through the glutathione ligands surrounding the Pd core. This indicates that the activity and selectivity of the NPs are strongly correlated to the interactions between the organic stabilizers and substrates. The recyclability of the catalysts for more branched substrates was poor, showing lower than $100 \%$ yield after the first cycle and then it decreased to nearly $75 \%$ after 5 cycles. The Pd NPs underwent some aggregation after the first cycle, but the selectivity of the catalyst basically remained unchanged, being more favorable 
for the isomerized product over all cycles tested. The catalytic activity of 2 years old synthesized PdAu NPs when compared with newly synthesized PdAu NPs for hydrogenation/isomerization of allyl alcohol showed the same reactivity with high stability, but the selectivity changed somewhat, which could be due to the loss of some ligands from the Pd surface with time.

In Chapter V, we synthesized naked Pd and CuPd nanoparticles stabilized by the citrate ligands and used those as pseudo-homogeneous catalysts for structurally similar allylic alcohols to determine their catalytic performances under biphasic conditions with dichloromethane and nanopure water solvents. In general, we observed that the catalytic activities of the NPs are strongly correlated with the structure of the substrate used whereas selectivity depends upon the kind of metal used. The CuPd NPs showed very high selectivity for the hydrogenated product but low TOF although the stabilizer was still citrate. This indicates that metal could impart product-based selectivity in the hydrogenation and isomerization of structurally related allylic alcohols.

In Chapter VI, we synthesized alkylamine-coated Pd NPs and checked their thermal stability for the Suzuki-Miyaura reaction. We observed that the longer C16 $\mathrm{NH}_{2}$ chain stabilized NPs were more stable at higher temperatures than $\mathrm{C} \mathrm{NH}_{2}$ and $\mathrm{C}_{12} \mathrm{NH}_{2}$ stabilized Pd NPs. $\mathrm{C}_{16 \mathrm{NH}_{2}}$ Pd NPs were used for the model Suzuki reaction of 4-iodoanisole and phenylboronic acid to form 4-methoxyphenyl benzene. Catalysis showed to be efficient to give $100 \%$ product within 4 hours.

Finally, Chapter VII summarizes the results of this research and suggests the future directions for it. 


\subsection{Motivation and Objective}

Performing efficient chemical transformations by designing novel catalytic systems, such as those involving metal nanoparticles (NPs), has been a significant recent goal in the modern science. Transition metal NPs have been widely used using solid supports for catalyzing a variety of organic reactions, ${ }^{1,2}$ but these heterogeneous systems typically suffer from the limitation of NP confinement, causing a negative impact on the turnover rates, limited lifetime and poor selectivity. ${ }^{3}$ This has directed studies on homogeneous systems, which potentially display higher activity and more controlled selectivity. ${ }^{4}$ These small homogeneous metal NPs are used to catalyze a variety of organic reactions. The key issue to get maximum efficiency in homogeneous catalysis with NPs is to control their size, shape, composition, stability, leaching of delicate core metals, imperfect recycling, and recovery of the metal NPs. These problems can be addressed by functionalizing them with organic stabilizers, such as polymers, ${ }^{5}$ surfactants, $^{6}$ ligands $^{7}$ or dendrimers, ${ }^{8}$ which passivate the surface and stabilize the NPs against aggregations. Although the functionalization stabilizes the NPs, it also greatly reduces the catalytic activity of the NPs by binding to the metal surface and poisoning the reactive sites. Also, since the metal NPs typically aren't stable in solvent-free form, it's very difficult to separate, recover and reuse these catalysts. ${ }^{9}$ Thus, our work was motivated by the need to find the stabilizers that allow high catalysis to number of organic catalytic conversions and highly stablilty against hydrogen or thermalinduced aggregation or morphological changes in solution. Also, those NPs should be easily recoverable and re-usable for cost-effectiveness. 


\subsection{Importance of Metal Nanoparticles}

Recent developments in nanotechnology have led to the synthesis of advanced materials using metal NPs with desirable reactivity as well as optical and electronic properties that have use in many applications, such as industrial, ${ }^{10}$ biomedical,11, 12 sensing, ${ }^{13,14}$ electronic applications, ${ }^{15}$ optical, ${ }^{16}$ electrochemical, ${ }^{17,} 18$ thermal, ${ }^{19}$ magnetic $^{20}$ and catalysis. ${ }^{21-25}$ Metal NPs are metal materials with at least one dimension in the 1-100 $\mathrm{nm}$ range. The unique electronic structure and properties of nanoparticles (NPs) are largely due to the high surface-to-volume ratio along with low coordination number of the surface atoms of NPs. This leads to sizedependent properties very different from the bulk materials. ${ }^{26,27}$ These properties brand NPs as a suitable candidate for the catalysis of a variety of organic reactions.

\subsection{Synthesis of Metal Nanoparticles}

Metal nanoparticles can be prepared by both physical and chemical methods. Figure 1.1. shows a schematic illustration of preparative methods of metal NPs. The physical typically involves vapor deposition methods, milling, mechanical grinding that depend on the mechanical sub-division of bulk precursors into nanoparticles. These methods generally yield larger NPs (>10 nm) with broad size distribution. ${ }^{28}$ The chemical approaches involve nucleation and growth of metallic atoms by reduction of metal ion precursors in the presence of some type of stabilizer, as shown in Figure 1.2.5, 26, 27 These methods are the most common ways to control the size of the NPs. Therefore, controlling the nucleation and growth of metal NPs are the most important challenges in controlling their size and uniformity. 
A variety of techniques have been introduced to prepare metal NPs, including ligand reduction and displacement from organometallics, ${ }^{5}$ sono-chemical reduction, ${ }^{29}$ chemical reduction of transition metals, ${ }^{5}$ decomposition of organometallic precursors ${ }^{30}$ and electrochemical deposition. ${ }^{31}$ The reduction of transition metal salts in solution by borohydride $\left(\mathrm{NaBH}_{4}\right.$ or $\left.\mathrm{KBH}_{4}\right)$ is the most widely used method of generating metal NP suspensions either in aqueous or organic phase. In general, the particles are formed by the reduction of metal ions in the presence of some stabilizers.

Typical coatings for stabilizing metal NPs include ligands, ${ }^{32-38}$ surfactants, ${ }^{39-}$ 41 polymers, ${ }^{42-45}$ ions, ${ }^{46-48}$ and dendrimers. ${ }^{22,}$ 49-52 Mayer and co-workers have studied extensively the stabilizing polymer on the reduction of an aqueous suspension of $\mathrm{Au}, \mathrm{Ag}$, $\mathrm{Pd}$ or Pt ions by $\mathrm{KBH}_{4}{ }^{53-56}$ They investigated polyacids, block copolymers, non-ionic polymers and cationic polyelectrolytes. $\mathrm{NaBH}_{4}$ has been used to obtain $\mathrm{Ag}, \mathrm{Au}, \mathrm{Pd}, \mathrm{Pt}$ or $\mathrm{Cu}$ NPs stabilized by dendrimers (polyamidoamine or PAMAM). ${ }^{22,49,57,58}$ These macromolecules lead to nearly mono-disperse particles. Surfactants are generally used as stabilizers of aqueous NP suspensions of transition metals reduced by $\mathrm{NaBH}_{4}$ or $\mathrm{KBH}_{4}$.

Ibañez and Zamborini synthesized tetraoctylammonium bromide (TOABr)coated $\mathrm{Pd}, \mathrm{Au}, \mathrm{AuAg}$ and $\mathrm{PdAg} \mathrm{NPs}$ by the reduction of $\mathrm{AuCl}_{4}, \mathrm{PdCl}_{4}^{2-}, \mathrm{Ag}^{+}$or some combination, with $\mathrm{NaBH}_{4}$ in the presence of the surfactant in toluene solutions ${ }^{59}$ which were used for studying gas ${ }^{60}$ and vapor ${ }^{60,61}$ sensing applications. Nakao and co-workers described the preparation of Rh, Ru, Pd, Pt, Au or Ag NPs stabilized by quaternary ammonium sulfates or poly (ethyleneglycol). ${ }^{62}$ Although, all of these 


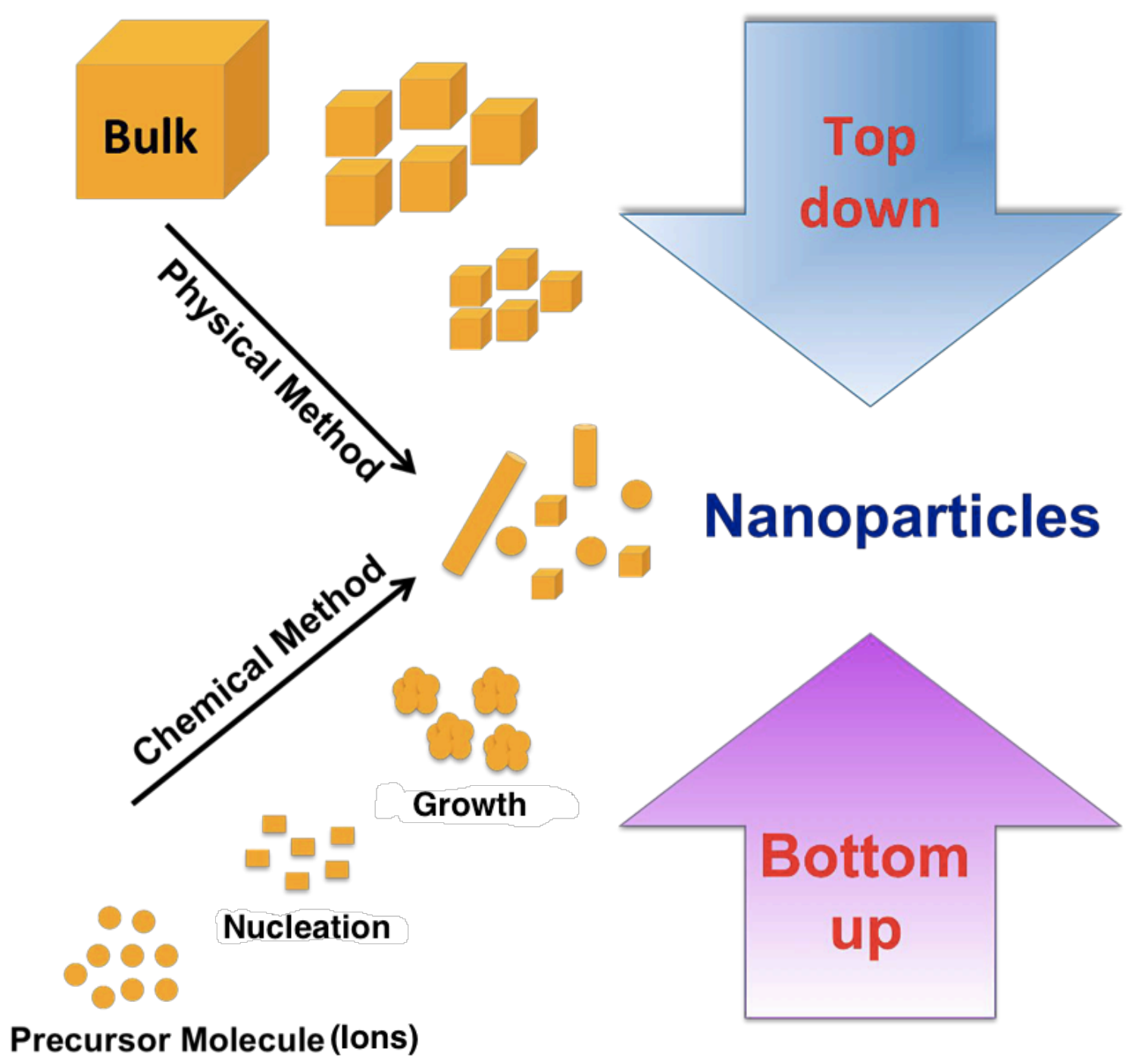

Figure 1.1. Schematic representation of synthesizing metal nanoparticles using physical or chemical methods. 


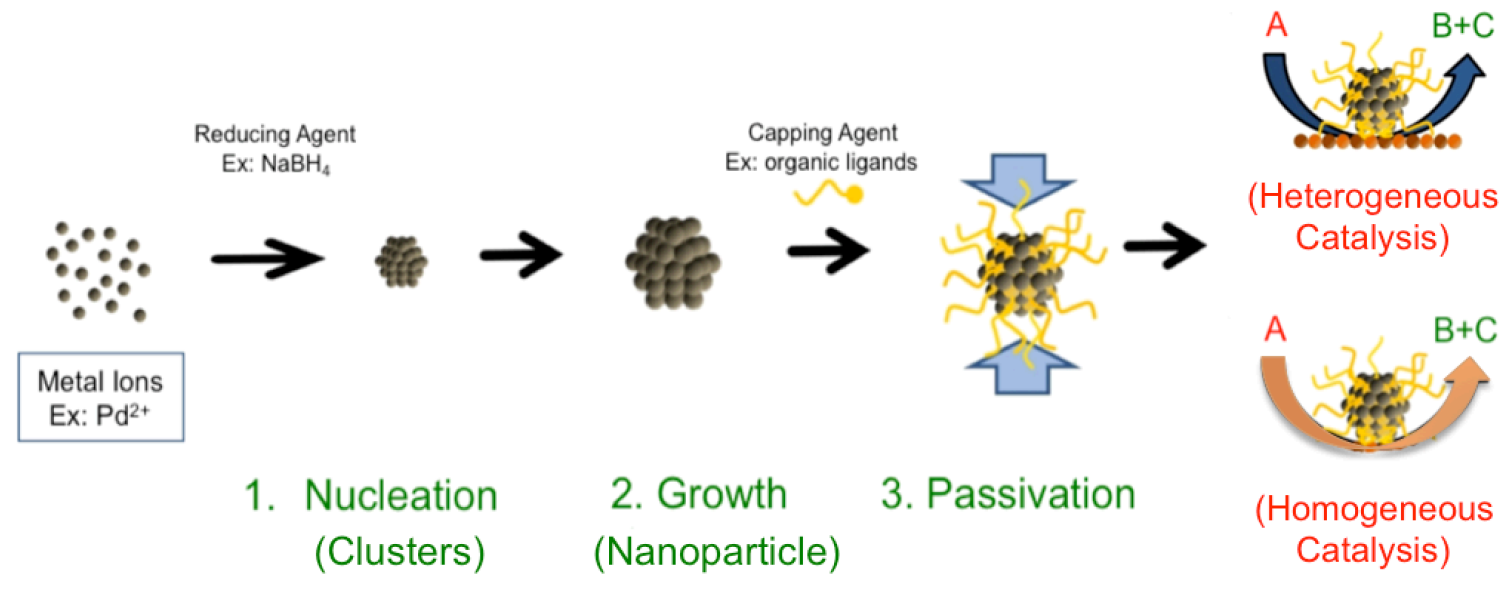

Figure 1.2. Schematic representation showing the nucleation, growth and passivation of metal to form stabilized metal NPs and its use in catalysis. 
capping stabilizers are efficient for the preparation of small and uniform NPs, the metal NPs are often stable only as suspensions and tend to irreversibly aggregate over time or when removed from solvent, which limits their manipulations, characterization, solubility, and potential uses. In Chapter II, we describe the synthesis of Pd, PdPt, PdAu and Cu@Pd NPs coated with various organic ligands, specifically, glutathione, trisodium citrate, and alkylamines, which can be repeatedly isolated from the solution mixture due to their use in a biphasic reaction. They can be reused for a number of reaction cycles without irreversible aggregation or decomposition.

\subsubsection{Metal Nanoparticles Coated with Organic Ligands}

In 1994, Brust and co-workers reported, for the first time, an easy and reproducible synthesis for the preparation of $\mathrm{Au}$ NPs stabilized with dodecanethiolate ligands. ${ }^{7}$ This procedure involves the transfer of tetrachloroaurate anions from an aqueous phase to an organic phase (toluene) by using a phasetransferring agent (tetraoctylammonium bromide). The addition of a solution of alkanethiol in toluene in the organic solution causes the reduction of $\mathrm{Au}^{\mathrm{III}}$ to $\mathrm{Au}^{\mathrm{I}}$ and formation of the $\mathrm{Au}^{\mathrm{I}}$-thiolate polymer. ${ }^{63}$ Subsequent reductive decomposition of the complex by $\mathrm{NaBH}_{4}$ leads to the NP formation by nucleation and growth while the thiolate ligands adsorb onto the surface of the NP to form a single self assembled monolayer (SAM) coating. ${ }^{14}$ Figure 1.3 shows a metal NP surrounded by various hydrophilic organic monolayers showing the possibility to tailor the metal composition through various stabilizers using the chemical synthesis. These NPs 
consist of roughly spherical metal cores surrounded by an organic monolayer.

Murray and co-workers have extensively studied the synthesis, reactivity and electrochemistry of Au NPs. ${ }^{23,64,65}$ They have shown that the thiol/Au mole ratios, temperature and rate of $\mathrm{NaBH}_{4}$ addition are critical parameters that are responsible for the core dimension and dispersity. In general, the NP size decreases with an increase in the thiol/Au ratio and generally have the range of 2 to $5 \mathrm{~nm}$ in diameter for alkanethiolate-protected Au NPs. ${ }^{66}$ Other reports in the literature have shown a wide variety of alkanethiolate chain-lengths (C3-C24), ${ }^{66} \omega$-functionalized alkanethiolates, ${ }^{67}$ dialkyl disulfides ${ }^{64}$ and aromatic thiols ${ }^{67}$ that can be utilized by employing the Brust-Schiffrin approach. It is also known that fast addition of reducing agent and using cooled solutions results in more monodisperse NPs. A few examples of alkanethiolate ligands used in nanoparticle synthesis include the hexanethiolate-coated (C6S) $\mathrm{Au}^{7} \mathrm{Pd}^{61}$ and Pt NPs, ${ }^{34}$ octanethiolate-coated (C8S) Pt NPs, ${ }^{68}$ nonanethiolate-coated (C9S) Ag NPs, ${ }^{69}$ and a mixture of alkanethiol and $\omega$ carboxylate alkanethiol (11-mercaptoundecanoic acid, MUA) NPs. ${ }^{70}$ The BrustSchiffrin procedure has also been extended to other sulfur-containing organic compounds such as thiolated $\beta$-cyclodextrins, ${ }^{32}$ sulfides, thioethers, ${ }^{71}$ disulfides $^{64}$ and Bunte salts $\left(\mathrm{RSSO}_{3} \mathrm{Na}^{+}\right) \cdot{ }^{72} \mathrm{~A}$ few other examples of organoamine ligands include octylamine-coated $\left(\mathrm{C} \mathrm{NH}_{2}\right)$, dodecylamine-coated $\left(\mathrm{C} 12 \mathrm{NH}_{2}\right)$, and hexadecylaminecoated $\left(\mathrm{C}^{16 \mathrm{NH}_{2}}\right) \mathrm{Au}^{73} \mathrm{Pd}$ and PdAg alloy $\mathrm{NPs}^{60,} 74-77$ and dodecylamine-coated

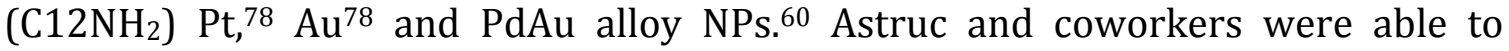
synthesize dodecanthiolate-stabilized PdNPs capable of catalyzing Suzuki-Miyaura coupling reaction ${ }^{36}$. From this work, they were able to successfully demonstrate for 


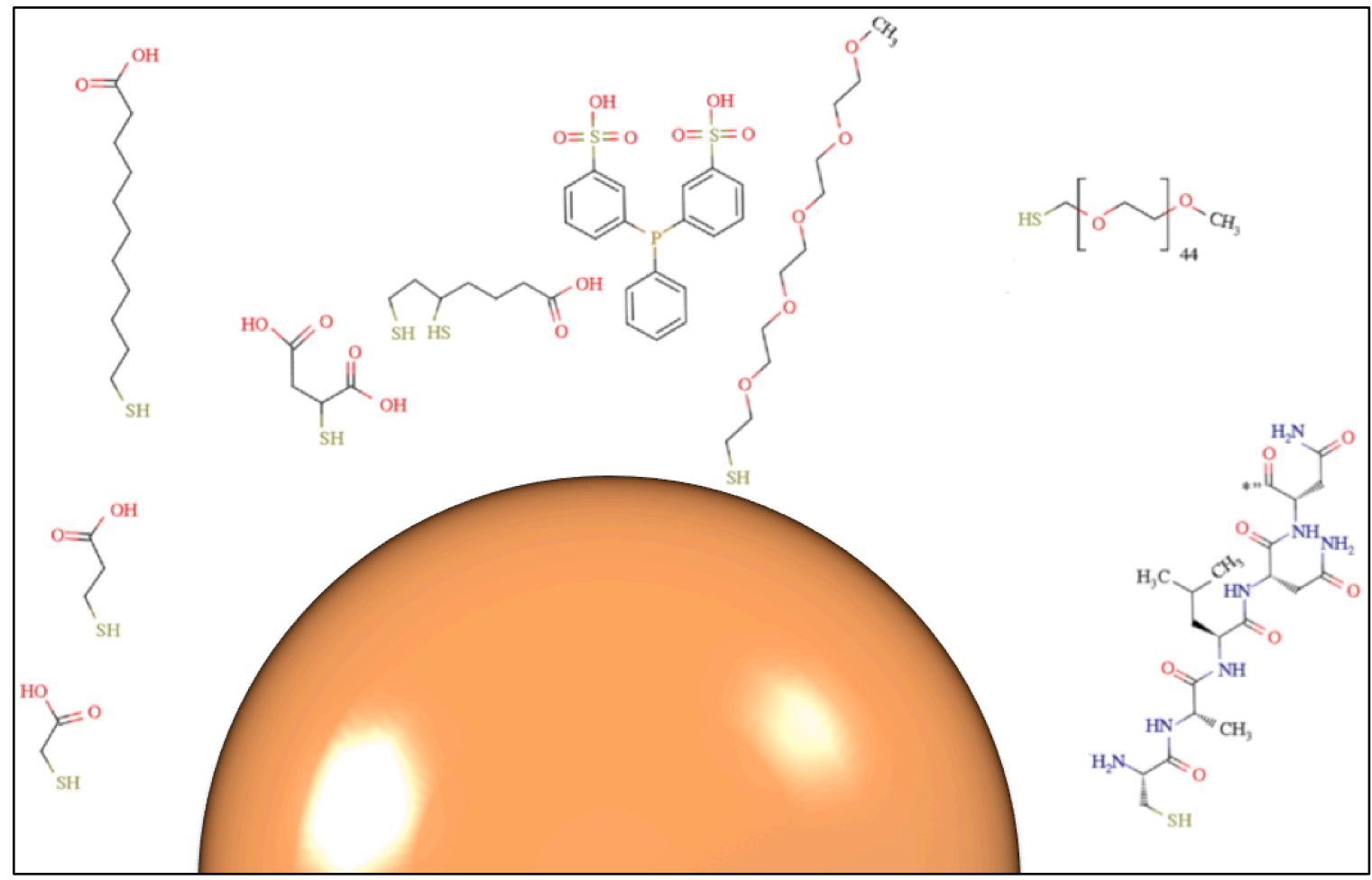

Figure 1.3. Schematic representation of a metal nanoparticle surrounded by various hydrophilic organic capping ligands. 
the first time that straight-chain alkanethiolate capped PdNPs could be used to catalyze a simple reaction under mild reaction conditions. These catalysts showed modest yields, but they were phosphine-free and recyclable. Later, Fornasiero and coworkers reported an easy approach for the preparation of functionalized Pd NPs by directly employing 11-mercaptoundecanoic acid (MUA) and mixed ligand monolayers of 9-mercapto-1-nonaol and 1-dodecanethiol as stabilizers and their easy recycling and reusability in Suzuki cross coupling reaction ${ }^{79}$. Recently, Shon and coworkers showed that alkanethiolate-coated Pd and PdAu NPs selectively catalyze the isomerization of allyl alcohol over the hydrogenation ${ }^{80,81}$.

\subsubsection{Metal Nanoparticles Stabilized Electrostatically}

In 1951, Turkevich pioneered the synthesis of Au NPs stabilized by citrate anions yielding mono-disperse, water-soluble NPs. ${ }^{46}$ These citrate-coated NPs are fairly naked where the stability of the nanoparticle solution is attributed to the collective effects of Van der Waals interactions, electrostatics and steric effects. ${ }^{82}$ Citrate has also proven to be a useful reagent in the synthesis of Pd nanomaterials ${ }^{47}$, 48,83 as shown in Figure 1.4, in addition to $\mathrm{Au}$ as seen in the literature. ${ }^{46}$ Han et al. reported a one-pot and seedless synthesis of core-shell Au-Pd bimetallic NPs via citrate co-reduction of $\mathrm{Au}$ and $\mathrm{Pd}$ ions, which catalyzed formic acid oxidation and Suzuki coupling reactions with better TOF in comparison to the $\mathrm{Au}-\mathrm{Pd}$ bimetallic NPs with other morphologies. ${ }^{84}$ Henglein and co-workers prepared Pd colloids with a narrow particle size distribution by the reduction of $\mathrm{PdCl}_{4}{ }^{2-}$ using hydrogen in aqueous solution with sodium citrate as the stabilizer. ${ }^{47}$ Huang et al. synthesized 
heterogeneous reduced graphene oxide (rGO) supported palladium nanoparticles (Pd NPs) with a size of $\sim 3 \mathrm{~nm}$ using one-pot photo assisted citrate reduction which exhibited 5.2 times higher mass activity for ethanol oxidation reaction than the commercial platinum/carbon (Pt/C) and comparable activity for oxygen reduction reaction. ${ }^{48}$ Shin and co-workers prepared heterogeneous $\mathrm{Ag} / \mathrm{Pd}$ alloy NPs by coreduction of Ag and Pd ions using citrate and attaching them on ITO and then examined their SERS activity and catalytic activity for the reduction of 4-nitrophenol to 4 -aminophenol by $\mathrm{NaBH}_{4}$ as a function of their metal composition. The SERS activity of $\mathrm{Ag} / \mathrm{Pd}(5 \%)$ particles was found to be an order of magnitude higher than that of pure Pd particles. Whereas, the catalytic activity of $\mathrm{Ag} / \mathrm{Pd}(5 \%) \mathrm{NPs}$ was the highest among $\mathrm{Ag}, \mathrm{Ag} / \mathrm{Pd}(2 \%), \mathrm{Ag} / \mathrm{Pd}(5 \%)$, and Pd NPs, suggesting a synergistic interaction between the Ag and Pd atoms ${ }^{83}$. Miroslav et al. synthesized stable Pd NPs with a tunable size $(3-15 \mathrm{~nm})$ by controlled chemical reduction of $\mathrm{PdCl}_{2}$ with sodium citrate in water. They showed that intensive stirring of the reaction mixture, controlled changes of $\mathrm{pH}$ and initial concentrations played a vital role in achieving reproducible particle sizes. ${ }^{85}$ These Pd NPs were conjugated with anti-mouse IgG antibody and used for ultrastructural immunolabeling, which confirmed Pd NPs suitability for multiple immunolabeling in biomedicine.

\subsubsection{Metal Nanoparticles as Homogeneous Catalysts}

In the past, various groups have studied the activity and selectivity for hydrogenation and isomerization of allyl alcohols using heterogeneous Pd catalysts, such as $\mathrm{Pd} / \mathrm{TiO}_{2},{ }^{86,87}$ and Pd NPs immobilized on composites or embedded in poly- 


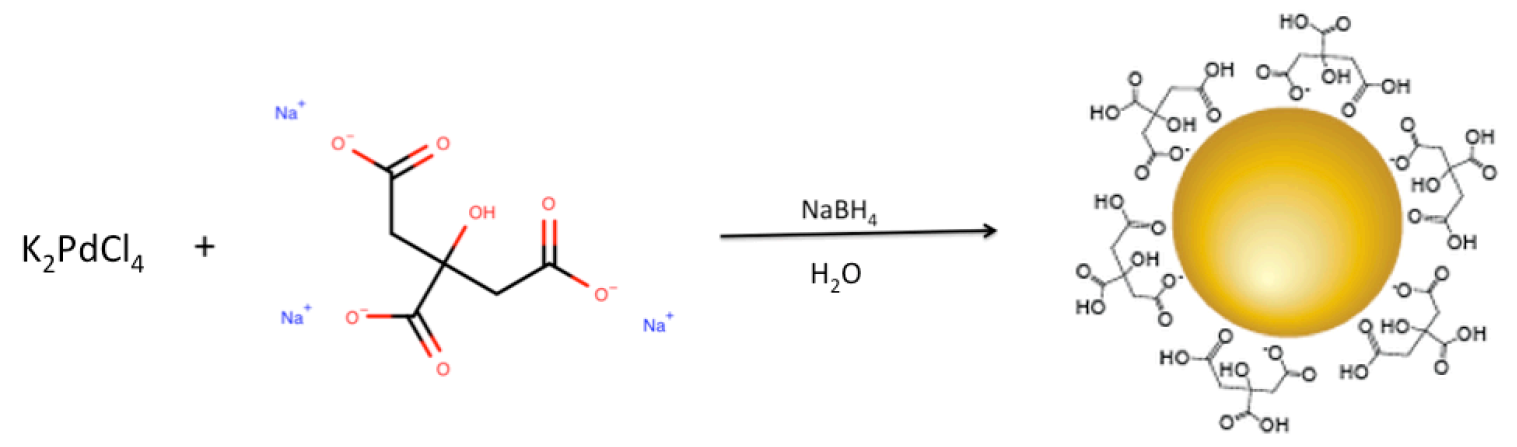

Figure 1.4. Schematic representation of a reaction involving the synthesis of citrate-capped Pd nanoparticles. 
electrolyte films (TOF=1600 \pm 190$) .88-91$ Here, we have focused our work on homogeneous catalysts. Shon and co-workers have reported the selectivity for isomer product in the hydrogenation/isomerization of allyl alcohol using dodecanethiolate-capped Pd NPs generated from thiosulfate where the reaction was $80 \%$ complete after one hour..$^{80,81}$

Zharmagambetova and co-workers reported the selectivity of $74 \%$ for isomer product by using Pd-polymer complexes containing iminodithiol as catalysts. ${ }^{92}$ Crooks and co-workers synthesized various Pd NPs encapsulated within poly(amidoamine) dendrimers functionalized with different-sized end groups and showed their size-selective catalytic activity for hydrogenation of allyl alcohol93,94 and C-C coupling reactions such as Heck ${ }^{95}$ and Stille ${ }^{57}$ reactions. They concluded that more steric crowding on the dendrimer surface led to lower TOF. ${ }^{94}$ Benefits of dendrimers include stability of NPs and imparting some selectivity by allowing molecules to access the NP surface. They concluded that more steric crowding on the dendrimer surface led to lower TOF.94 They also showed that Pd-Pt dendrimerencapsulated NPs have higher catalytic activity (TOF=193) than dendrimerencapsulated Pd or Pt NPs (TOF=50) for hydrogenation of allyl alcohol. ${ }^{96}$. Zamborini and co-workers synthesized Pd NPs stabilized with hexanethiol (TOF=47) and alkylamines of various chain lengths (TOF=719 for hexadecylamine-coated Pd NPs) and investigated their catalytic activity and selectivity for the hydrogenation/ isomerization reaction of allyl alcohol. ${ }^{75}$ Recently, Shon and co-workers used a biphasic catalyst of water-soluble alkanethiolate-capped Pd NPs with hydrophobic active sites in the aqueous phase. Both the $\mathrm{pH}$ of the NPs solution and size of the 
substrates were important factors in determining the activity and selectivity of Pd NPs. ${ }^{97}$ Using a biphasic solvent system in catalytic reactions is advantageous as the products can be easily removed from the organic phase and the catalyst dissolved in the aqueous phase can be reused several times without precipitating and redissolving.

\subsection{Catalysis with Chemically Synthesized Metal Nanoparticles}

Metallic Pd materials with nanoscale dimensions have been widely studied as a potential catalyst for a variety of organic reactions including olefin hydrogenation and C-C bond synthesis. The presence of defect sites on the surface of NPs causes inhomogeneous coverage of the ligands. ${ }^{98}$ These sites include vertices, edges and grain boundaries on the NP surface. In these areas, the ligands are less crowded and less organized and thus, has higher surface energy making them more reactive. This gives the substrates more opportunity to interact with the NPs and results in the formation of product as compared to reactions on the terrace and smooth regions of the NP, where the ligands are well packed, organized and crowded.65, 98 Research on metal NPs has attracted a great deal of attention in recent years, specially in the fabrication of $\mathrm{Pd}$ nanostructures including nanowires, ${ }^{99}, 100$ nanotubes, ${ }^{101}$ nanochains ${ }^{102}$ and nanoparticles ${ }^{13,103}$ due to their use in the applications that involve interactions with hydrogen. The initial study on palladium-hydrogen $(\mathrm{Pd}-\mathrm{H})$ system was intensively done by Lewis in $1974 .{ }^{104}$ In Chapters II, IV and V, we study the interactions of hydrogen with Pd and Pd-alloy NPs coated with various organic and electrostatic ligands in solutions and explore their applications in the pseudo- 
homogeneous catalysis of olefins. Metallic Pd materials with nanoscale dimensions have also been widely studied as a potential catalyst for a variety of other organic reactions, including C-C bond synthesis, which we will discuss in Chapter VI.

\subsubsection{Palladium-Hydrogen system}

Since hydrogen is going to be one of the reactants in the hydrogenation/ isomerization reaction of allyl alcohol, it is very important for the metal nanoparticles to be stable under a hydrogen atmosphere. Figure 1.5 shows the adsorption and dissociation of $\mathrm{H}_{2}$ onto the Pd surface. When Pd is exposed to hydrogen gas, Pd catalyzes the reaction where molecular hydrogen gets reversibly adsorbed to the surface of Pd. It then rapidly dissociates and diffuses into the metal lattice as atomic $\mathrm{H}$ to form $\mathrm{PdH}_{\mathrm{x}}$ and occupies octahedral interstitial sites. ${ }^{105}$ This increases the Pd lattice constant. The reactions are given below.

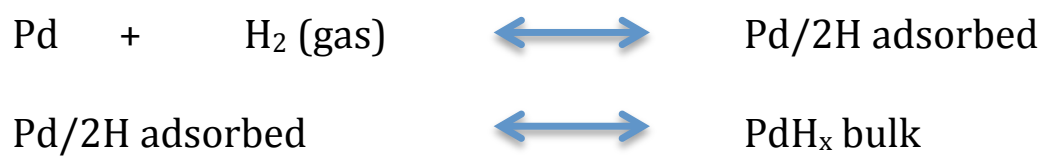

Diffusion of atomic hydrogen at low $\mathrm{H}_{2}$ partial pressure into the Pd lattice causes Pd to undergo a phase transition to form $\alpha$-phase $\mathrm{PdH}_{\mathrm{x}}$, which expands at high $\mathrm{H}_{2}$ partial pressure to form $\beta$-phase $\mathrm{PdH}_{\mathrm{x} .}{ }^{106}$ (Figure 1.5.) This phase transition and resulted increase in the lattice constant alters the properties of the Pd and its alloy NPs thus forming the basis of catalysis. ${ }^{106,107}$ The resulting Pd-Pd atomic spacing on the material also alters the volume, ${ }^{108}$ the resistance ${ }^{109-111}$ and the 
electrical ${ }^{112-118}$ and the optical ${ }^{119-121}$ properties of the metal. The diffusion of hydrogen into the Pd lattice provides large hydrogen storage capacity, and the dissociation of hydrogen by breaking hydrogen $1 \mathrm{~s}-1 \mathrm{~s}$ bonds into the more active atomic hydrogen leads to the desirable catalytic properties as shown in Figure 1.6.

\subsection{Catalysis and Metal Nanoparticles}

In all catalytic reactions, metal NPs are generally either used as heterogeneous catalysts where they are attached to a solid support or homogenous catalysts where they are soluble in solution. Although the use of transition metal nanoparticles in catalysis has been frequently performed over superficial solid support for a variety of organic and inorganic reactions, ${ }^{1,2,122}$ since they can be easily recovered from the reaction and reused several times, these heterogeneous systems suffer from the limitation that nanoparticles are jammed to a solid support causing negative impact on the turnover rates, limited lifetime and poor selectivity primarily due to the principle of diffusion and the involvement of two different phases (solid-liquid) in the catalytic system. ${ }^{123}$ Also, their very complex method of preparation and poor particle morphology control ${ }^{21,124}$ has redirected studies on the homogeneous systems. These homogeneous systems have recently drawn great interest as a potential catalytic system with potentially higher activity and better selectivity. ${ }^{4,125}$ Although most of the studies for homogeneous catalysis have been conducted using NPs coated with polymers, dendrimers or surfactants, 5,6 these metal NPs are unstable in solution and undergo changes in their morphology in the reaction conditions. 


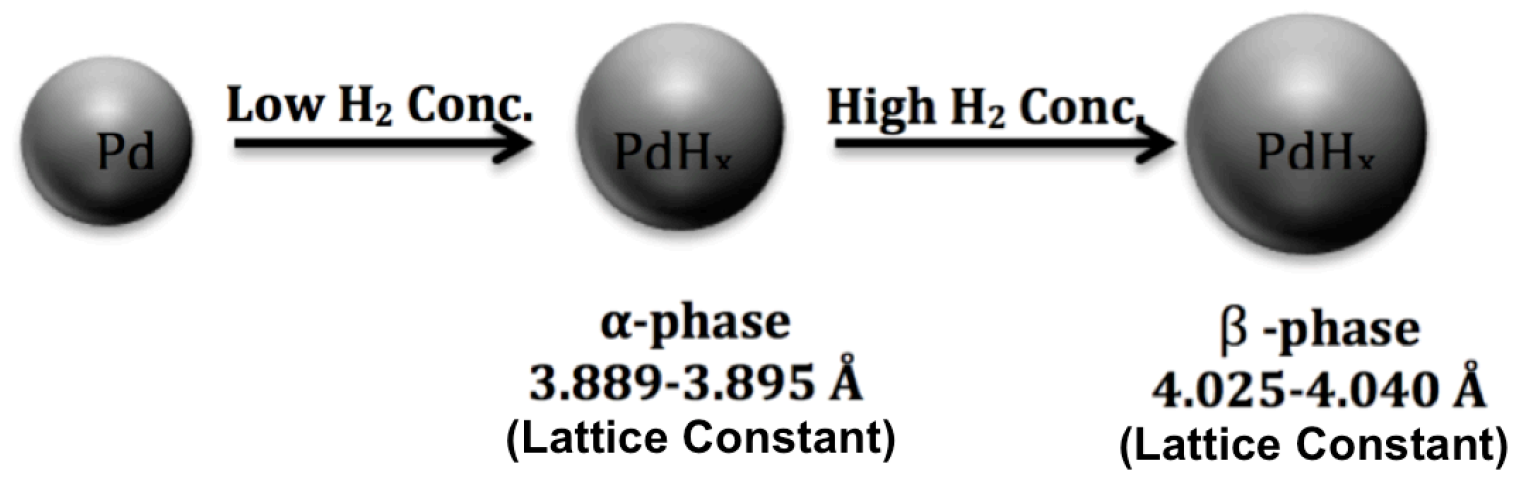

Figure 1.5. Illustration of $\mathrm{H}_{2}$ adsorption into the $\mathrm{Pd}$ lattice and diffusion causing phase transition from $\alpha$-phase $\mathrm{PdH}_{\mathrm{x}}$ lattice to $\beta$-phase $\mathrm{PdH}_{\mathrm{x}}$. 


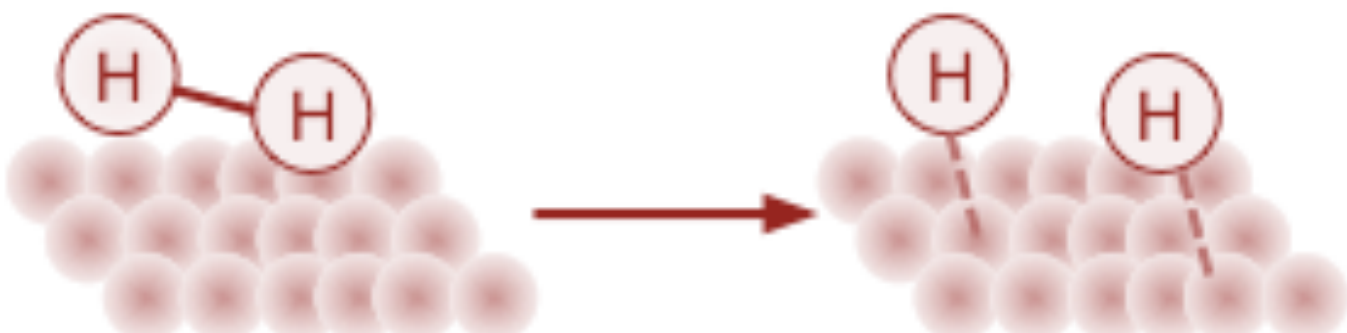

Figure 1.6. Dissociation of hydrogen by breaking $1 \mathrm{~s}-1 \mathrm{~s}$ bonds into the more active atomic hydrogen on the Pd surface leading to the desirable catalytic properties. 
Also, since some of these colloidal NPs aren't stable in solvent-free form, it's very difficult to separate, recover and reuse these catalysts. ${ }^{9}$ Some of the more current issues involving homogeneous catalysis include pollution impact due to the use of toxic organic solvents, leaching of delicate core metals, and imperfect recycling and recovery of metal NPs. ${ }^{126}$

There is a need to investigate and develop highly stable and reactive homogeneous catalysts that shows higher recyclability potential. A popular remedy for this problem is the introduction of organic-ligand stabilizers, such as thiols, ${ }^{34}$ phosphines, ${ }^{127}$ amines, ${ }^{75}$ ammonium salts, ${ }^{128}$ selenolates, ${ }^{129}$ and isocyanides ${ }^{130}$ prior to or during the nanoparticle nucleation-growth stage. These organic-ligand stabilizers make a monolayer coated on the core consisting of metallic atoms and provide control over the core size of the nanoparticles, depending on their ratio during synthesis. These ligands also function as gates/filters that selectively allow reagents to reach the active surface of the nanoparticles in a particular orientation. ${ }^{8}$ This accounts for the observed selectivity and turnover frequency of the nanoparticles. These NPs are usually highly stable and can be stored without solvents. They are easy to characterize and can be easily separated from the products by precipitation. However, these types of NPs haven't been studied much since these surface passivants are usually strongly bound to the metal surface and poison the reactive sites, thus, lowering the catalytic activity significantly. ${ }^{131}$

Metal NPs has been used for determining their catalytic activity using hydrogenation/isomerization of allyl alcohols as a model reaction. This reaction is simple and helps to determine the selectivity of the NPs by producing one of the two 
products selectively. Various strategies have been reported in the literature to use metallic NPs for catalyzing this organic reaction. For example, Sadeghmoghaddam et al. focused on understanding the mechanism and regioselectivity of Pd NPs in different environments resulting in both hydrogen and type of solvent being essential for the catalytic isomerization reaction. ${ }^{132}$ Eklund et al. synthesized several platinum NPs coated with different thiol functionalized organic-soluble alkyl chains and water-soluble ligands and used them for hydrogenating allyl alcohols to propanol and maleic acid to succinic acid. The differences in hydrogenation rates were attributed to variations in ligand chain-length, branching, charged functional groups, packing density and core-size. ${ }^{34}$ Shon and co-workers reported the synthesis of dodecanethiolate capped Pd NPs, by employing a Bunte Salt precursor, for the hydrogenation of allyl alcohol.133 They also provided examples that alkanethiolate-coated Pd and PdAu NPs selectively catalyze the isomerization of allyl alcohol over hydrogenation. ${ }^{80,81}$ The same group recently investigated the biphasic catalysis of water-soluble alkanethiolate-capped Pd NPs as structurally stable micelle catalysts in aqueous phase for the hydrogenation and isomerization of allyl alcohols. Both the $\mathrm{pH}$ of the nanoparticle solution and the size of the substrates were shown to be important factors in determining the activity and selectivity of the

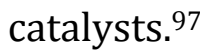

Marshall et al. described the synthesis of highly selective Pd catalysts involving the deposition of $\mathrm{n}$-alkanethiol self assembled monolayer coatings that improves the selectivity of 1-epoxybutane formation from 1-epoxy-3-butene from 11 to $94 \%$ at equivalent reaction conditions using traditional platinum group 
catalysts. ${ }^{134}$ Zaera et al. reported the significant improvement in both activity and selectivity during the hydrogenation of $\alpha$-keto esters with cinchonidine as a chiral modifier by the addition of alkyl thiol self-assembled monolayers to the colloidal platinum NPs which were explained by a kinetic effect in which the cinchonidine residence time on the surface was increased by the thiol self-assembled layer. ${ }^{135}$

Other reports relevant to our work here include the use of glutathione ligands for preparing NPs anchored on metal oxides such as $\mathrm{ZnO}$ and $\mathrm{TiO}_{2}$ to prepare a composite catalyst ${ }^{136}$ and clinical drugs for cancer therapy. ${ }^{137}$ There has also been significant progress in the synthesis and application of monodispersedglutathione capped $\mathrm{Au}^{138-140}$ and $\mathrm{Ag}^{137,141,142}$ NPs with definite core size, but much less progress on the synthesis and applications of glutathione-capped Pd NPs. ${ }^{143}$

In this dissertation, we focus on the use of organic ligand-stabilized Pd NPs as catalysts as they are highly stable, can be easily characterized and stored as solids and electrostatically stabilized Pd NPs which are naked and thus, highly reactive. Also, all these NPs have the potential to get easily separated from the reaction products by either precipitation or by using a biphasic solvent mixture and thus, help in getting better recyclability.

\subsubsection{Importance and Reactivity of Allyl Alcohol with Hydrogen}

The reaction of allyl alcohol with hydrogen in the presence of metal NPs as catalysts results in either or both 1-propanol and propanal. The hydrogenated product (1-propanol) is a precursor in the organic synthesis of various natural products and in the pharmaceutical industry and has been studied as a function of 
shape and size of Pd NPs coated with polymer, 88,144 biopolymers (collagen fiber),145 dendrimers $50-52,93,94,146,147$ either in solution or immobilized on solid support such as alumina, ${ }^{41}, 88$ composites, $^{52}$ silica ${ }^{148}$ and magnetic NP cores $^{149-151}$ and surfactants. ${ }^{41}$ However, the isomerization of allyl alcohol to carbonyl products has not been studied well using Pd NPs. ${ }^{152}$ Since, the isomerization reaction is an important one-pot catalytic transformation to carbonyl compounds that proceeds with high efficiency, selectivity and atom economy, avoiding the two-step sequential oxidation and reduction reactions, formation of higher concentration of isomerized product is one of the main advantages. The isomerization reaction has been carried out under harsh conditions using expensive metals in high quantities till now. 153 Sensitive substrates do not survive the conditions involving the use of toxic and/or expensive oxidizing and reducing agents and therefore, catalytic redox isomerization is especially useful in their synthesis under mild reaction conditions as shown in Figure 1.7. Here, there are two mechanisms shown through which the isomerization product can form using allyl alcohol. In mechanism A, the alcohol group on the substrate allyl alcohol can be oxidized to form a carbonyl and then reduction of the double bond occurs resulting in the isomerized product. In mechanism B, the double bond of the allyl alcohol is reduced first and then the alcohol group can oxidize to the carbonyl, leading to the formation of 1-propanal. Both mechanisms lead to the same isomer end product.

Also, in terms of atom economy, redox isomerization is an attractive strategy as no byproducts are formed, only saturated aldehyde or ketones are formed, which are valuable intermediates for various pharmaceuticals, agrochemicals and fine 


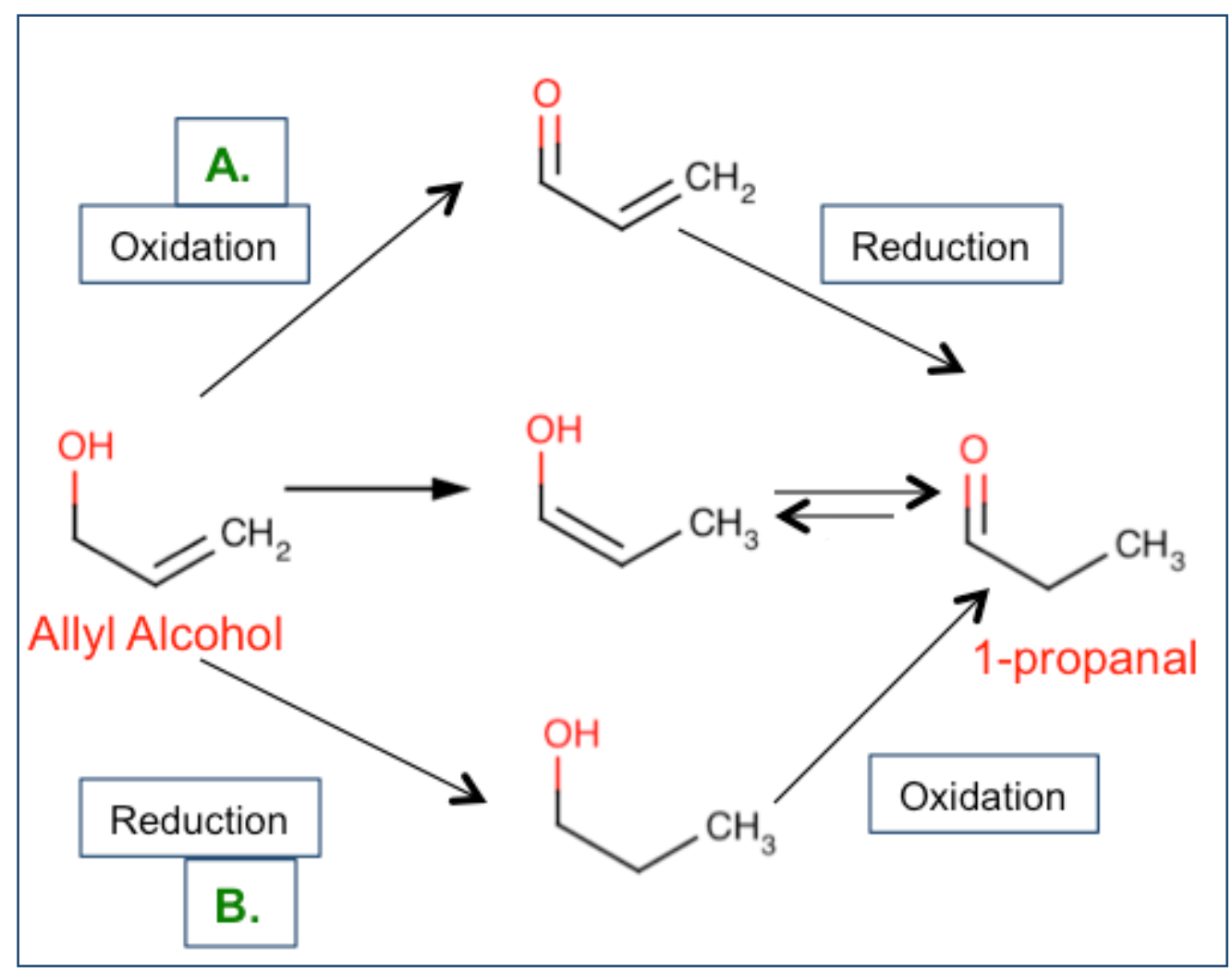

Figure 1.7. Schematic illustration of the catalytic redox isomerization reaction using allyl alcohol. 
chemicals. $^{154}$ These isomerization reactions can be used in a multi-step synthesis of high value added compounds in organic chemistry like naturally occurring pheromones like muscone,155 (+)-iso-exo-brevicomin, ${ }^{156}$ the marine alkaloid (-)brevisamide, ${ }^{153}$ the fragrance Florhydral, ${ }^{157}$ and the antitumor agent (-)FR182877,158 all of which includes a redox isomerization step. Although several transition metals have been used to perform this reaction, ruthenium, rhodium and iron have shown the best results in activity and selectivity. In this dissertation, we have shown that Pd NPs stabilized with glutathione ligands and Cu@Pd-citrate NPs have great importance for this reaction due to their high selectivity towards isomerization, excellent stability, high surface-to-volume ratios, and highly active surfaces.

\subsubsection{Mechanism of hydrogenation and isomerization of Allyl Alcohol}

The mechanism for the hydrogenation and isomerization of allyl alcohol has proposed during the course of time, ${ }^{154,159-161}$ which mainly includes the Pd-alkyl intermediate formation. It is an intermolecular mechanism that requires the presence of hydrogen to form $\mathrm{Pd}-\mathrm{H}$ species, either isolated or prepared in situ before the adsorption of allyl alcohol and initiation of the catalytic reaction. The $\pi$ coordination of the allyl alcohol substrate to the $\mathrm{Pd}-\mathrm{H}$ rich surface is followed by the formation of a Pd-alkyl intermediate. Ultimately, the selectivity of the reaction for either isomerization or hydrogenation products depended on the regioselective insertion of the $\mathrm{Pd}-\mathrm{H}$ to the $\mathrm{C}=\mathrm{C}$ bond. These reactions are reversible and thus, the abstraction of the hydrogen $\alpha$ to the $\mathrm{OH}$ group leads to the formation of an enol and 
complexed to the metal hydride. A final decomplexation gives back the catalyst and enol, which then tautomerizes to the carbonyl derivative. ${ }^{154}$ Shon and co-workers showed that the higher steric interference to the Pd core causes the preferable formation of a linear Pd-alkyl (anti-Markornikov addition) intermediate, promoting the hydrogenation of allyl alcohol to 1-propanol. The least amount of steric hindrance during the insertion step allows the formation of a branched Pd-alkyl (Markornikov addition) intermediate and promotes the isomerization of allyl alcohol to propanal ( $\beta$-elimination) as shown in Figure 1.8. (A).161

Another known mechanism for the isomerization of allyl alcohol is known as $\pi$-allyl hydride mechanism, which is an intramolecular mechanism that doesn't require the presence of hydrogen since the $\mathrm{Pd}-\mathrm{H}$ adduct is generated from the oxidative addition of $\mathrm{Pd}$ to the $\mathrm{C}-\mathrm{H}$ bond $\alpha$ to the $\mathrm{OH}$ group as shown in Figure 1.8. (B). ${ }^{159}$ In Chapters IV and V, we evaluate the catalytic properties of various allyl alcohol using Pd and Pd-alloy NPs for the hydrogenation and isomerization reaction of allyl alcohol in the presence of hydrogen and thus, they follows the Pd-alkyl mechanism. In our research, we are the first to show the use of glutathione-coated and citrate-coated Pd and Pd-alloy NPs for the hydrogenation and isomerization of allyl alcohol under a biphasic solvent system.

\subsubsection{Importance of Suzuki-Miyaura reaction and its mechanism}

The Suzuki cross-coupling reaction of arylboronic acids and aryl halides provides an effective synthetic route to form biaryls. ${ }^{162-164}$ The coupling reaction of 

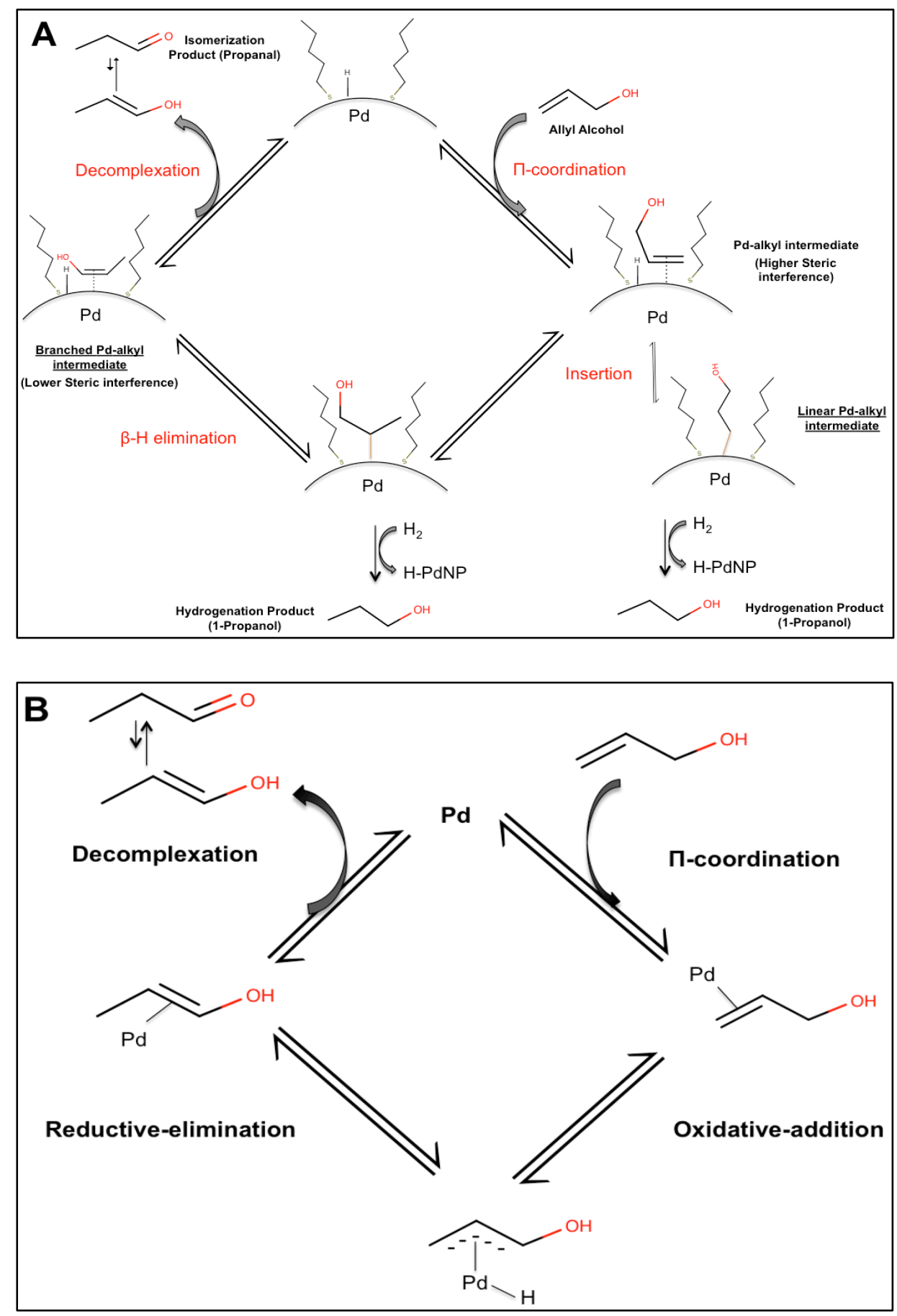

Figure 1.8. Proposed mechanisms for the hydrogenation and isomerization as (A) Pd-alkyl mechanism and (B) $\pi$-allyl hydride mechanism for the isomerization reaction of allyl alcohol. 
arylboron derivatives with aryl halides in the presence of $\mathrm{Pd}\left(\mathrm{PPh}_{3}\right)_{4}$ and base to afford biaryls was first reported ${ }^{165}$ in 1979 . Since then, a number of modifications have appeared. Suzuki coupling reactions present wide applications in the production of polymers, poly-olefins, styrene, substituted biphenyls, agrochemicals, pharmaceutical intermediates, and high-tech materials. ${ }^{166,167}$ It is a key coupling reaction in the synthesis of a variety of marketed products including the blood pressure medications valsartan and losartan, the antifungal agrochemical boscalid, and even the OLEDs (Organic Light-Emitting Diodes) used in TVs and monitors. ${ }^{166}$ The widespread use of the Suzuki cross-coupling reactions is mainly due to the mild conditions associated with these reactions, their tolerance to a wide variety of functional groups, availability of diverse boronic acids, and the easy handling and removal of boron-containing byproducts when compared to other organometallic reagents. These reactions are carried out in organic solvents and catalyzed by various Pd NPs and Pd(II) complexes. ${ }^{168}$ Colloidal metal particles are effective catalysts for the chemical transformations due to their large surface area. ${ }^{169}$ It has been shown that Pd colloids on the nanometer length scale are effective catalysts for the Suzuki reactions ${ }^{170}$ in organic solvents due to their milder reaction conditions as well as more environmentally friendly methods. Nevertheless, one serious problem in homogeneous metal catalysis is separation of the reaction products from the catalysts.

Reetz and co-workers were the first to report the use of Pd and Pd/Ni NPs for the Suzuki coupling of aryl bromides and chlorides with phenylboronic acid using 2 mol\% of these catalysts. ${ }^{170}$ El-Sayed and co-workers initially reported the use of Pd 
NPs stabilized by PVP as catalysts in the coupling of aryl iodides with phenylboronic acid in aqueous media. ${ }^{171}$ The rate of the reaction was shown to depend on the catalyst concentration, indicating that the Suzuki reaction occurred at the metallic surface. El-Sayed and co-workers also reported the use of Pd NPs stabilized by poly(amido-amine) dendrimers of different generations (D2-D4) bearing hydroxyl groups and compared the stability of the resulting NPs by measuring the amount of Pd black formed during the reaction. 3, 43, 172,173 The nanoparticles stabilized by a mixture of polymer D3 and PVP were the most efficient catalysts. Astruc and coworkers synthesized dodecanthiolate-stabilized PdNPs capable of catalyzing SuzukiMiyaura coupling reaction of alkyl bromide and iodide under ambient conditions. ${ }^{36}$ From this work, they were able to successfully demonstrate for the first time that straight-chain alkanethiolate capped PdNPs could be used to catalyze a simple reaction under mild reaction conditions. These catalysts showed modest yields, but they were phosphine-free and recyclable. Later, Fornasiero and coworkers reported an easy approach for the preparation of functionalized Pd NPs by directly employing 11-mercaptoundecanoic acid (MUA) and mixed ligand monolayers of 9-mercapto-1nonanol and 1-dodecanethiol as stabilizers and their easy recycling and reusability in Suzuki cross coupling reaction..$^{79}$ Fox and co-workers reported the catalytic behavior, including for the Suzuki-Miyaura reaction, of Pd NP-cored dendrimers. ${ }^{174}$ The Kaifer group ${ }^{175}$ and Newmann group ${ }^{176}$ reported the catalytic activity in the Suzuki-Miyaura reactions of thiolate-Pd NPs modified, respectively, with $\beta$ cyclodextrin and polyoxometallates.

The mechanism of the Suzuki-Miyaura reaction is best viewed by looking at 
the Pd catalyst as shown in Figure 1.9.162 The first step involves the Oxidative Addition of Pd to the halide 2 to form the organopalladium species 3. In most cases, the oxidative addition is the rate-determining step of the catalytic cycle. After that, reaction with base gives the intermediate 4 , which via transmetallation with the boronate complex 6 forms the organopalladium species 8. Transmetallation is an organometallic reaction where ligands are transferred from one species to another. Boroante complex 6 is formed by the reaction of boronic acid 5 with base. Reductive elimination of the desired product 9 restores the original palladium catalyst 1 which completes the catalytic cycle. Also, understanding the nature of the active sites that drive the chemical transformations has been an important but often elusive goal in catalytic studies. In recent years, two different mechanisms for catalyzing Suzuki reactions using metal nanoparticles have emerged, depending upon the homogeneous or heterogeneous nature of the Pd NPs ${ }^{177}$ as shown in Figure 1.10. Pd atoms and ions leach from Pd clusters under Suzuki coupling conditions and the leaching mechanism would depend on the reaction conditions. In the absence of any oxidizing agent, $\mathrm{Pd}(0)$ atoms would leach out from the surface into the solution entering into the cross-coupling cycle. Accordingly, a homogeneous mechanism for the Pd-catalyzed C-C bond forming reaction is proposed. However, in the presence of iodobenzene, Pd(II) complexes would be formed by the oxidative addition of the aryl halide to Pd atoms, either on the cluster surface (heterogeneous mechanism) or previously leached into the solution (homogeneous mechanism). Thus, the formed Pd(II)-complex would enter into the catalytic cycle either directly or after being leached out into the solution. According to these works, no catalytic activity would 
be found on the Pd cluster surface. Even though only re-deposition is displayed in Figure 1.8, re-clustering to form new smaller particles and/or formation of palladium black have been reported, depending on the reaction conditions. ${ }^{177}$

The conclusions in the literature differ as to whether the Pd NP catalysis of the Suzuki reaction arises from leached metals or nanoparticles themselves. Also, it is possible that the Suzuki cross-coupling reaction may operate under homogeneous or heterogeneous catalytic conditions, depending on a variety of factors such as the nature of the Pd NPs and their stabilizers as well as the properties of the reactants involved and the working conditions. In this case, rather than mutually exclusive, both mechanisms are considered as complementary and dependent on the immobilization degree of Pd NPs where the Pd atoms mobilize out of the core or used as such in catalysis. 


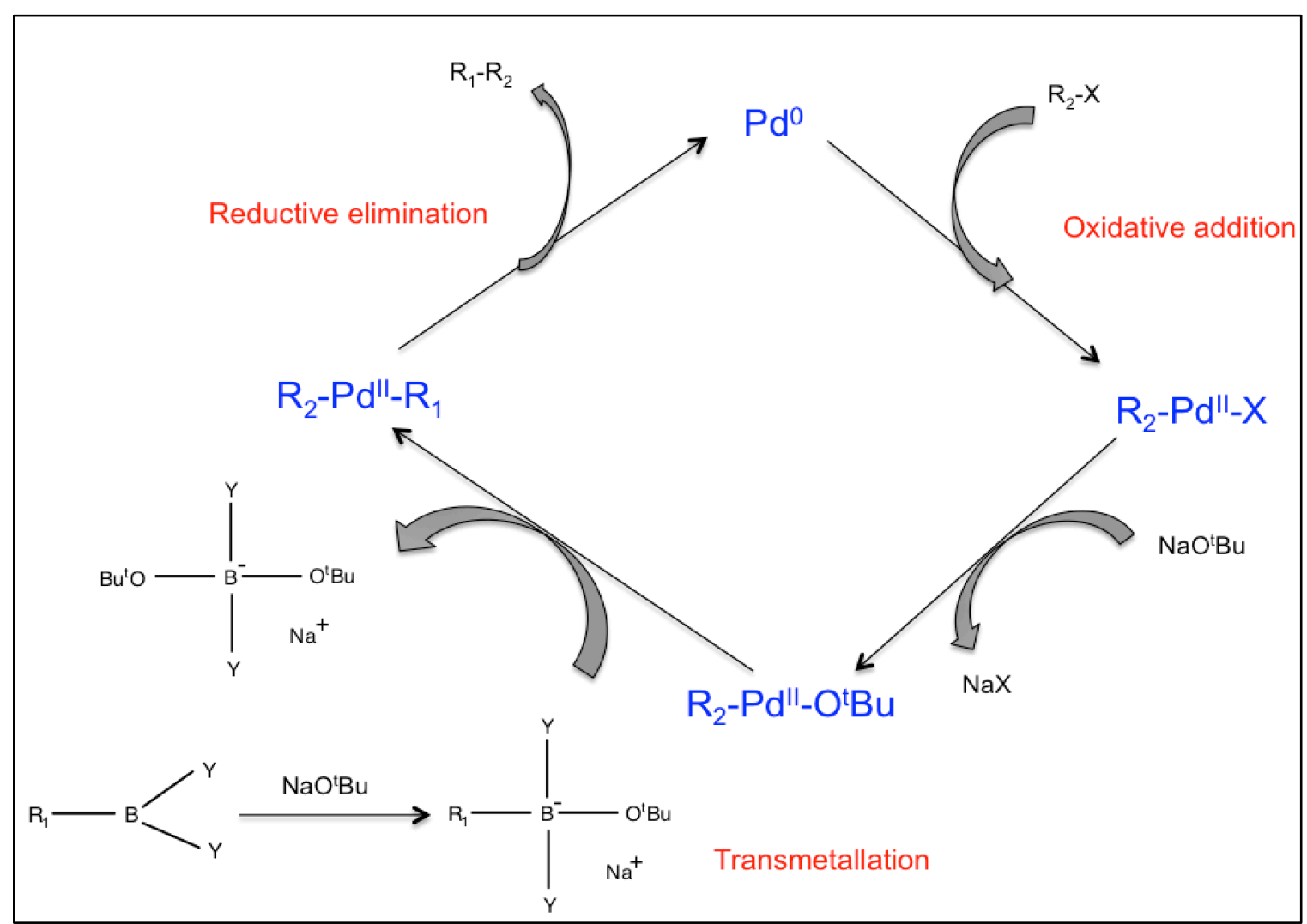

Figure 1.9. Mechanism for the Pd catalyzed Suzuki cross-coupling involving three main steps. 


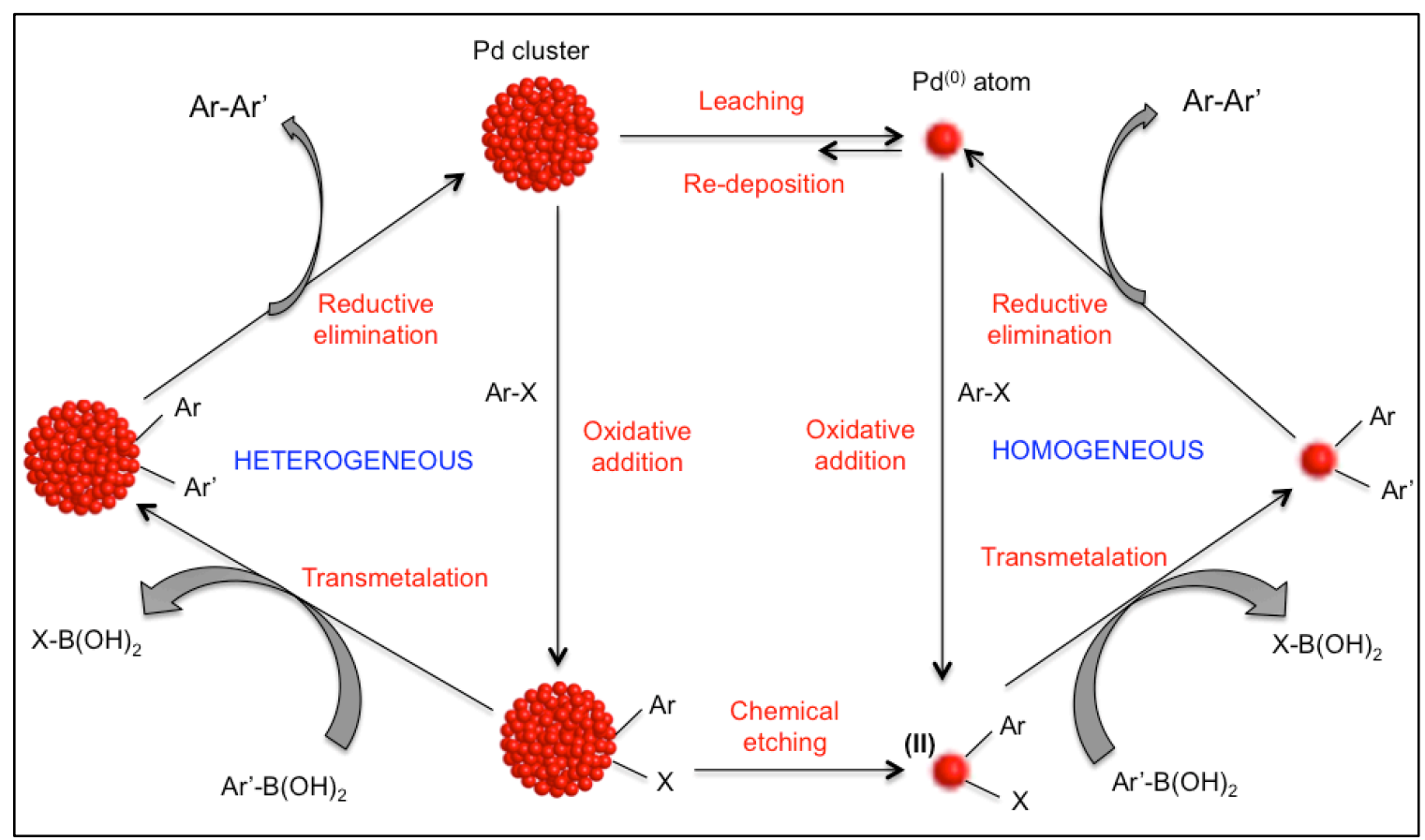

Figure 1.10. Proposed mechanisms for the Pd NP-catalyzed Suzuki crosscoupling involving both homogeneous and heterogeneous pathways. 


\section{CHAPTER II}

\section{EXPERIMENTAL}

This chapter includes six main experimental parts.

1) Chemicals used

2) Chemical synthesis of various Pd, PdPt, PdAu and CuPd Nanoparticles (NPs)

3) Nanoparticle characterization

4) Hydrogen stability studies of solutions of NPs

5) Catalysis studies for the hydrogenation/isomerization of various olefins under biphasic solvent system

6) Catalysis studies for the Suzuki-Miyaura reactions

\subsection{Chemicals}

Dichloromethane (99.5\%), toluene (99.9\%), acetonitrile (99\%), ethanol (200 proof), methanol (99\%), acetone (99\%) and 2-propanol (99.9\%) were purchased from VWR Scientific Products and used as received. Sodium borohydride (98\%), potassium tetrachloroplatinate(II), Reduced-L-glutathione, ethyl acetate (99\%), allyl alcohol (99\%), propyl alcohol (99.7\%), dodecylamine (98\%), propionaldehyde (99\%), 1-propen-3-ol (99.5\%), 1-hepten-3-ol (99.5\%), and 2-methyl-3-buten-2-ol (99.9\%) were purchased from Aldrich Chemical Co. and used as received. 
Tetraoctylammonium bromide (98\%), octylamine (99\%), 1-hexadecyl amine (90\%) containing 1-octadecylamine, potassium tetrachloropalladate(II) (99\%), chlorobenzene (98\%), 4-iodoanisole (99.5\%), phenylboronic acid (99.5\%), and potassium carbonate (99\%) were purchased from Alfa Aesar Co. and used as received. Hydrogen tetrachloroaurate(III) was synthesized in our lab from 99.99\% Au. Deuterium oxide and chloroform-d were purchased from Cambridge Isotope Laboratories. Ultra-high purity $\mathrm{H}_{2}$ gas $(99.999 \%)$ was used as received. Water was purified using a Barnstead nanopurification water system $(18.3 \mathrm{M} \Omega \mathrm{cm})$ and used for all aqueous solutions.

\subsection{Synthesis of Pd, PdPt, PdAu and Cu@Pd Nanoparticles (NPs)}

\subsubsection{Synthesis of Glutathione-Coated Pd, PdPt and PdAu (Inert) NPs}

We followed the synthesis reported by Sharma et al143. The synthesis was carried out in inert nitrogen atmosphere in order to avoid the possible oxidation of Pd nanoparticles. In the synthesis of "Inert" glutathione-coated Pd NPs, $0.50 \mathrm{~g}$ (1.531 mmol) of $\mathrm{K}_{2} \mathrm{PdCl}_{4}$ in $30 \mathrm{~mL}$ of nanopure water was dissolved and nitrogen gas was purged for at least 1 hour. Separately, $0.165 \mathrm{~g}(0.536 \mathrm{mmol})$ of reduced-Lglutathione in a molar ratio of 1:3 for Pd:thiol was dissolved in $5 \mathrm{~mL}$ of nanopure water and purged in nitrogen for 1 hour. The two solutions were then combined and stirred under a nitrogen atmosphere until $\mathrm{PdCl}_{4}{ }^{2-}$ formed a complex with glutathione as indicated by the change in color from yellow to wine red color as shown in Figure 2.1. The reaction mixture was further stirred at $360 \mathrm{rpm}$ for 
another 1 hour, which was then cooled down using an ice-bath. Subsequently, a fresh aqueous solution of $\mathrm{NaBH}_{4}$ was prepared by dissolving $0.58 \mathrm{~g}$ (15.31 mmol) of $\mathrm{NaBH}_{4}$ in $5 \mathrm{~mL}$ of nanopure water that was already purged in nitrogen for 1 hour. This solution was immediately added to the reaction mixture under stirring, giving an immediate change in color from wine red to black, indicating the formation of Pd nanoparticles. The solution was further stirred for 4 hours from which Pd NPs were separated by adding an equal volume of methanol $(40 \mathrm{~mL})$, stirring for 15 minutes, and centrifuging at $4000 \mathrm{rpm}$ for 10 minutes to obtain precipitates of the Pd NPs. The mother liquor was removed and the precipitated Pd NPs were dissolved in nanopure water and transferred into a round bottom flask to remove the water using a rotary evaporator. The black NPs were suspended in $100 \mathrm{~mL}$ of methanol and collected by vacuum filtration on a glass-fritted Büchner funnel. The NPs were then washed thoroughly with methanol, ethanol and acetone successively two times before thoroughly drying and collecting. These NPs are referred to as Pd-Glu (Inert) NPs in this dissertation.

The average diameter of Pd-Glu (Inert) NPs was 1.1-2.8 nm. ${ }^{143}$ The thermo gravimetric analysis (TGA) of Pd and Pd-alloy NPs leads to thermal decomposition of organic stabilizers leaving Pd as the residual mass. The TGA for Pd-Glu (Inert) NPs showed $25.6 \%$ organic and 74.4\% Pd metal as shown in Figure 2.3. (A). Frame (A) exhibits the TGA of pure Pd-Glu (inert) NPs with a fairly rapid $8.7 \%$ weight loss occurring between 100 and $200^{\circ} \mathrm{C}$ and additional gradual weight loss up to $800^{\circ} \mathrm{C}$, resulting in a total weight loss of $25.6 \%$. The UV-vis spectra exhibit an absorbance throughout the entire wavelength range that decays exponentially with increasing 

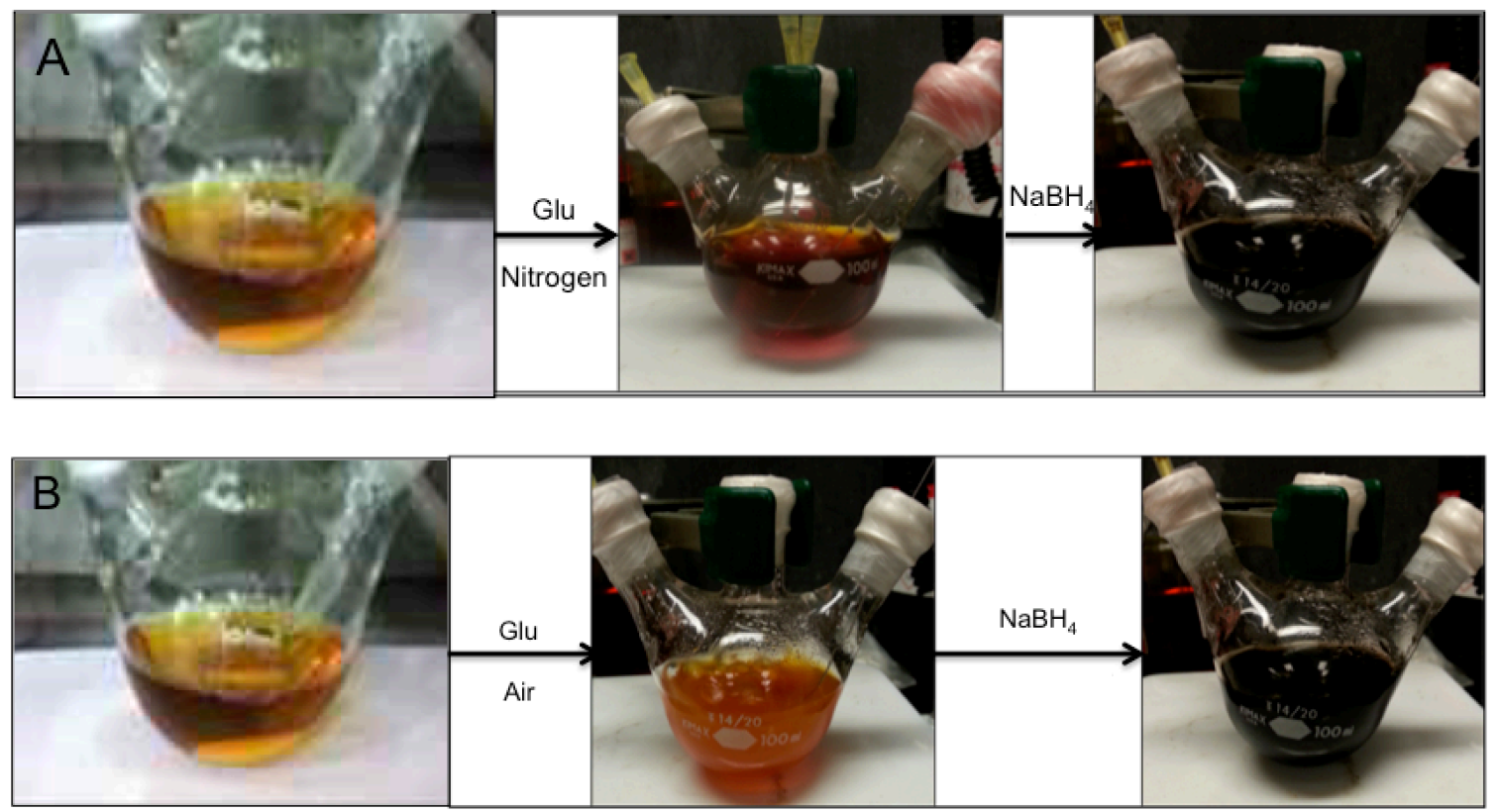

Figure 2.1. Digital pictures of steps in solution phase synthesis of Pd-Glu (Inert) NPs (A) and Pd-Glu (Oxy) NPs (B). 
wavelength, which is expected for metallic $\mathrm{Pd}^{0}$ nanoparticles. Figure 2.2. illustrates a metal NP obtained as a product from the organic synthesis and an example of the chemical properties of Pd-Glu (Inert/Oxy) NPs.

Pd-alloy NPs with different ratios of Pt or Au were synthesized ranging from 90:10, 75:25 and 50:50 under inert conditions by co-reducing them using $\mathrm{NaBH}_{4}$. Here, both $\mathrm{K}_{2} \mathrm{PdCl}_{4}$ and $\mathrm{K}_{2} \mathrm{PtCl}_{4}$ or $\mathrm{HAuCl}_{4} \cdot 3 \mathrm{H}_{2} \mathrm{O}$ metal salts in their respective molar ratios (90:10, 75:25 and 50:50) were dissolved together in nanopure water and used for further synthesis as described above.

\subsubsection{Synthesis of Glutathione-Coated Pd, PdPt and PdAu (Oxy) NPs}

Pd NPs coated with glutathione ligands under "Oxygen" conditions were prepared similarly by performing the above experiment under air atmosphere without purging nitrogen gas and keeping the molar concentration of $\mathrm{K}_{2} \mathrm{PdCl}_{4}$, reduced-L-glutathione and $\mathrm{NaBH}_{4}$ the same. The only visible difference was the formation of an orange colored solution during the addition of glutathione solution to the Pd salt solution as shown in Figure 2.1. These NPs are referred to as Pd-Glu (Oxy) NPs in this dissertation. The average diameter of Pd-Glu (Oxy) NPs was 1.7$3.7 \mathrm{~nm} .{ }^{143}$ The thermogravimetric analysis (TGA) showed $19.0 \%$ organic and $81.0 \%$ Pd metal for Pd-Glu (Oxy) NPs as shown in Figure 2.3. (B). Frame B shows the thermal decomposition of the organic portion of Pd-Glu (Oxy) NPs. The most rapid weight loss occurs between $300-400^{\circ} \mathrm{C}$ with a total weight loss of $19.0 \%$ up to $800^{\circ} \mathrm{C}$. The weight loss between 200 and $800^{\circ} \mathrm{C}$ occurs in three fairly discrete steps with steep regions and plateaus in the TGA plot that is fairly similar compared to the 


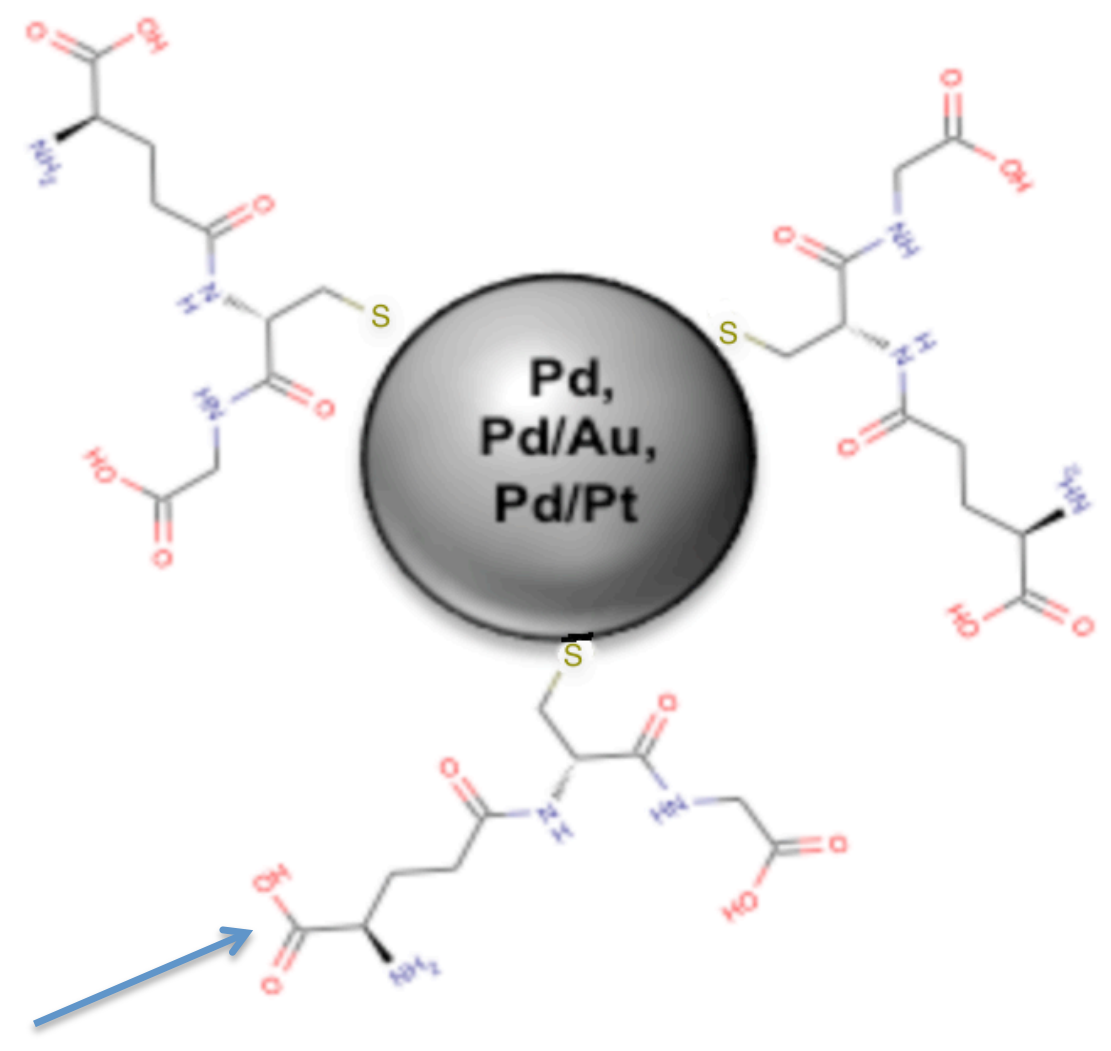

\section{Organic Stabilizer}

\section{Typical Example for Pd-Glu (Inert/0xy) NPs:}

(For 1:3 Pd:Glu mole ratio)

${ }^{143}$ Average Core Diameter: 1.1-2.8 nm for Pd-Glu (Inert) NPs

$$
\text { 1.7-3.7 nm for Pd-Glu (Oxy) NPs }
$$

TGA Analysis: $25.6 \%$ organic - 74.4\% Pd metal for Pd-Glu (Inert) NPs 19.0\% organic - 81.0\% Pd metal for Pd-Glu (Oxy) NPs

Figure 2.2. Schematic representation of glutathione-coated Pd, PdPt or PdAu NPs obtained as a product of synthesis. 

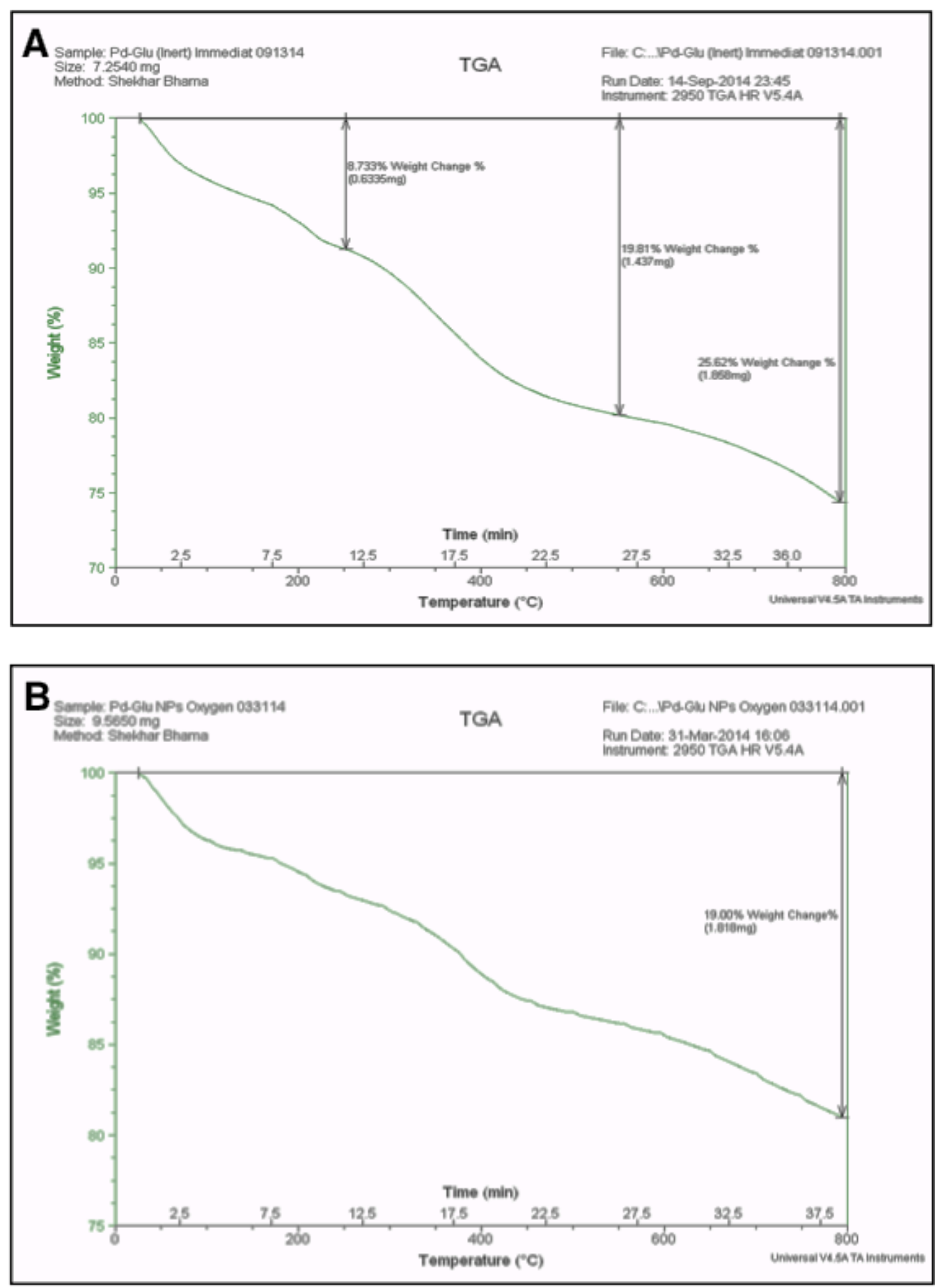

Figure 2.3. Thermogravimetric (TGA) measurements of (A) Pd-Glu (Inert) NPs and (B) Pd-Glu (Oxy) NPs. 
gradual weight loss observed for Pd-Glu (Inert) NPs throughout the entire 200$800^{\circ} \mathrm{C}$ range. The UV-vis spectra exhibit an absorbance throughout the entire wavelength range that decays exponentially with increasing wavelength, which is expected for metallic $\mathrm{Pd}^{0}$ nanoparticles.

Pd-alloy NPs with different ratios of Pt or Au were synthesized ranging from 90:10, 75:25 and 50:50 under oxygen conditions by co-reducing them using $\mathrm{NaBH}_{4}$. Here, both $\mathrm{K}_{2} \mathrm{PdCl}_{4}$ and $\mathrm{K}_{2} \mathrm{PtCl}_{4}$ or $\mathrm{HAuCl}_{4} \cdot 3 \mathrm{H}_{2} \mathrm{O}$ metal salts in their respective molar ratios (90:10, 75:25 and 50:50) were dissolved together in nanopure water and used for further synthesis as described above. Figure 2.4. shows a digital picture of Pd-Glu (Oxy) NPs in solid state and dissolved in water.

\subsubsection{Synthesis of Citrate-Coated Pd and CuPd core-shell NPs}

We modified the synthesis reported by Zhang et al. ${ }^{178}$ and used it for synthesizing citrate-coated Pd NPs. In a typical experiment, $30 \mathrm{~mL}$ of nanopure water was cooled to $0^{\circ} \mathrm{C}$ using an ice bath in a $100 \mathrm{~mL}$ round bottom flask and degassed by purging nitrogen gas for 30 minutes and used further. $20 \mathrm{~mL}$ of the above water was added with aqueous solution of $\mathrm{K}_{2} \mathrm{PdCl}_{4}(50 \mu \mathrm{L}, 0.1 \mathrm{M})$ and trisodium citrate $(50 \mu \mathrm{L}, 0.1 \mathrm{M})$ under constant nitrogen purging and ice-cold condition to another $50 \mathrm{~mL}$ round bottom flask and stirred for 5 minutes. Afterwards, $1 \mathrm{~mL}$ of freshly prepared $\mathrm{NaBH}_{4}$ (3.8 mg in $4 \mathrm{~mL}$ nanopure water) was injected into the reaction mixture rapidly. The color of the reaction mixture turned dark brown indicating the formation of Pd NPs. The reaction mixture was stirred for 30 minutes at ice-cold temperature and then stored in a vial at ambient temperature 


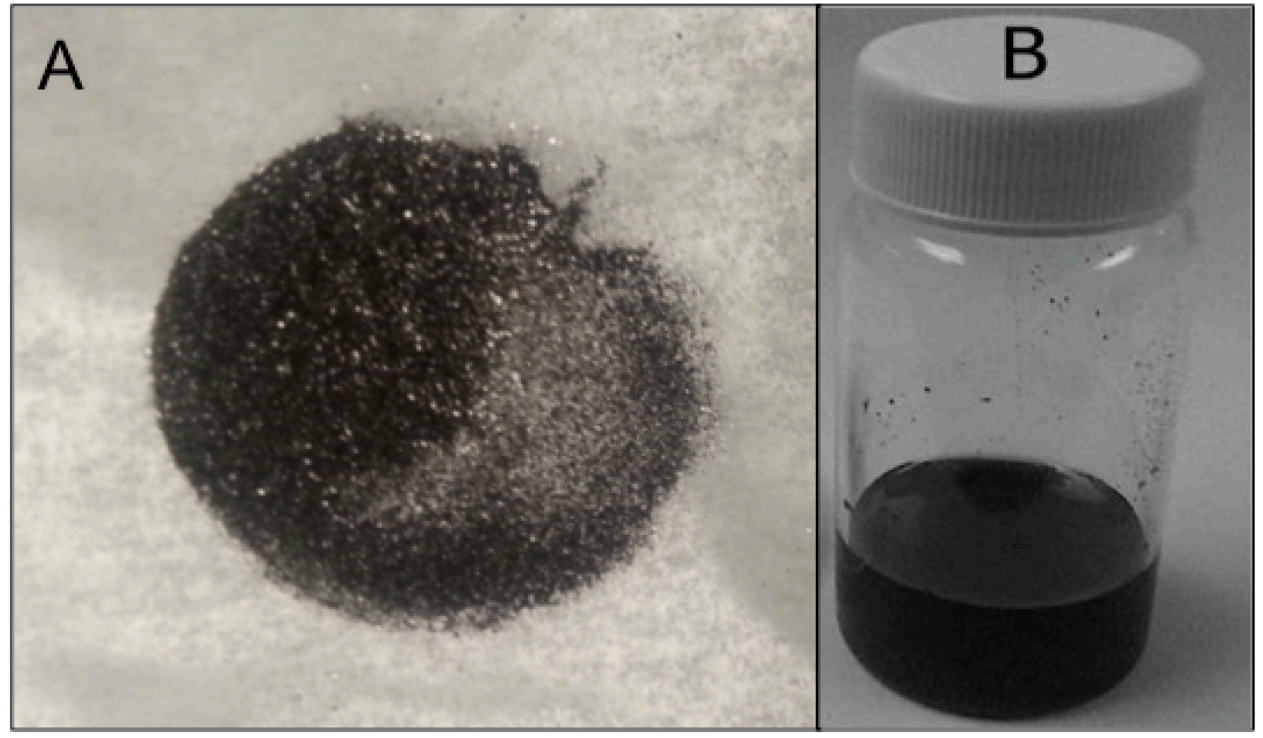

Figure 2.4. Digital pictures of Pd-Glu (Oxygen) NPs in solid state (A) and dissolved in nanopure water (B). 
for 24 hours before its further applications. These NPs are referred to as Pd-citrate NPs. The UV-vis spectra exhibited an absorbance throughout the entire wavelength range that decays exponentially with increasing wavelength, which is expected for metallic $\mathrm{Pd}^{0}$ nanoparticles.

For the synthesis of citrate-coated CuPd core-shell NPs, typically, $30 \mathrm{~mL}$ of nanopure water was cooled to $0^{\circ} \mathrm{C}$ using an ice bath in a $100 \mathrm{~mL}$ round bottom flask and degassed by purging nitrogen gas for 30 minutes and used further. $20 \mathrm{~mL}$ of the above water was added with aqueous solution of $\mathrm{CuSO}_{4} \cdot 5 \mathrm{H}_{2} \mathrm{O}(50 \mu \mathrm{L}, 0.1 \mathrm{M})$ and trisodium citrate $(50 \mu \mathrm{L}, 0.1 \mathrm{M})$ under constant nitrogen purging and ice-cold condition to another $50 \mathrm{~mL}$ single necked round bottom flask and stirred for 5 minutes. Then, $1 \mathrm{~mL}$ of freshly prepared $\mathrm{NaBH}_{4}$ (3.8 $\mathrm{mg}$ in $4 \mathrm{~mL}$ nanopure water) was injected into the reaction mixture rapidly. The color of the reaction mixture turned dark brown. The reaction mixture was stirred for 15 minutes at ice-cold temperature and then, $\mathrm{K}_{2} \mathrm{PdCl}_{4}(100 \mu \mathrm{L}, 0.1 \mathrm{M})$ was added to the solution mixture and stirred for another 20 minutes. The reaction mixture was stored in a vial at ambient temperature for 24 hours before its further applications. These NPs are referred to as CuPd-citrate NPs. The UV-vis spectra didn't show any plasmon for $\mathrm{Cu}$ and exhibited an absorbance throughout the entire wavelength range that decays exponentially with increasing wavelength, which is expected for metallic $\mathrm{Pd}^{0}$ nanoparticles. 


\subsubsection{Synthesis of Alkylamine-Coated Pd NPs [octylamine $\left(\mathrm{C}^{2} \mathrm{NH}_{2}\right)$, dodecylamine $\left(\mathrm{C12NH}_{2}\right)$, hexadecylamine $\left.\left(\mathrm{C16NH}_{2}\right) \mathrm{Pd} \mathrm{NPs}\right]$}

Syntheses of Alkylamine-coated Pd NPs at room temperature were reported previously. ${ }^{60,74,75}$ Briefly, solutions of $0.50 \mathrm{~g}(1.53 \mathrm{mmol})$ of $\mathrm{K}_{2} \mathrm{PdCl}_{4}$ in $10 \mathrm{~mL}$ of nanopure water and $1.67 \mathrm{~g}(3.06 \mathrm{mmol})$ of $\mathrm{TOABr}$ in $70 \mathrm{~mL}$ of toluene were combined and stirred until all $\mathrm{PdCl}_{4}{ }^{2-}$ transferred into the toluene phase. Then, the appropriate ligand was added in a 12:1 alkylamine:Pd mole ratio and rapidly stirred for 45 minutes. This ratio corresponds to $18.4 \mathrm{mmol}$ of alkylamine, which is $2.97 \mathrm{~mL}$

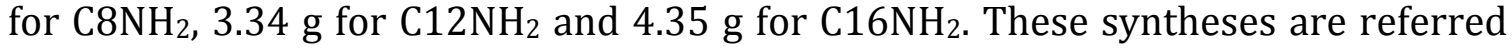
to as $\mathrm{C} \mathrm{NH}_{2} \mathrm{Pd}, \mathrm{C}_{12} \mathrm{NH}_{2} \mathrm{Pd}$ and $\mathrm{C}_{16 \mathrm{NH}_{2}} \mathrm{Pd} \mathrm{NPs}$. The organic layer became light yellow whereas the aqueous layer turned cloudy white upon addition of the alkylamine. This could be due to the complexation between the protonated amine and $\mathrm{PdCl}_{4}{ }^{2-}$, but it is not certain since this precipitate has not been characterized. ${ }^{76}$ Afterwards, $0.87 \mathrm{~g}$ of $\mathrm{NaBH}_{4}(23.0 \mathrm{mmol})$ in $10 \mathrm{~mL}$ of water was added to the twophase solution while stirring. The organic phase quickly turned black and cloudiness in the aqueous phase disappeared after about 2 min. The solution was stirred for 3 hour, the clear and colorless water layer was separated, and the toluene layer was later removed by evaporation. The black Pd NP product was suspended in $100 \mathrm{~mL}$ of acetonitrile by sonication and allowed to settle overnight before collecting by vacuum filtration. The resulting NPs were washed with acetonitrile and ethanol to remove excess ligands and other reaction byproducts before collecting. For $\mathrm{C}_{12} \mathrm{NH}_{2}$ Pd and $\mathrm{C}_{16 \mathrm{NH}_{2}} \mathrm{Pd} \mathrm{NPs}$, the solid was re-dissolved in toluene and the solvent reduced in volume again by rotary evaporation. The black NPs were precipitated in 
acetone overnight and the precipitate collected by vacuum filtration before washing with acetone and ethanol. All three $\mathrm{CnNH}_{2}$ Pd NPs were black powders and soluble in non-polar solvents. The average diameters of $\mathrm{C} \mathrm{NH}_{2} \mathrm{Pd}, \mathrm{C} 12 \mathrm{NH}_{2} \mathrm{Pd}$ and $\mathrm{C} 16 \mathrm{NH}_{2}$ Pd NPs were 3.5, 3.4 and $3.0 \mathrm{~nm}$, respectively. ${ }^{75}$

The thermogravimetric analysis (TGA) for $\mathrm{C} \mathrm{NH}_{2} \mathrm{Pd}, \mathrm{C} 12 \mathrm{NH}_{2} \mathrm{Pd}$ and $\mathrm{C} 16 \mathrm{NH}_{2}$ Pd NPs showed 19.0\% : 81.0\%, 22.0\% : 78.0\% and 77.1\%: 22.9\% organic and Pd metal, respectively. Here, TGA for $\mathrm{C}_{16 \mathrm{NH}_{2}} \mathrm{Pd}$ NPs is shown in Figure 2.5, which shows the thermal decomposition of the organic portion of $\mathrm{C}_{16 \mathrm{NH}_{2}} \mathrm{Pd}$ NPs. The most rapid weight loss occurs between $300-400^{\circ} \mathrm{C}$ with a total weight loss of $22.9 \%$ up to $800^{\circ} \mathrm{C}$. The organic content for $\mathrm{C}^{16 \mathrm{NH}_{2}}$ Pd NPs (77.1\%) is too large for a single monolayer and is attributed to the formation of a full or partial bilayer of ligands on the Pd core as reported previously by Shuang and co-workers for octadecylaminecoated Pd NPs. ${ }^{35}$ Table 2.1. shows all the different types of synthesis performed in this dissertation. The table summarizes various metal salts used, type of stabilizer, metal/stabilizer ratios, reducing agent and type of solvent used for dissolving NPs.

\subsection{Nanoparticle Characterization}

\subsubsection{Thermo-gravimetric Analysis (TGA)}

In TGA, the weight of NPs is measured as a function of temperature. At some temperature where the entire organic portion of the NP is thermally decomposed, the organic percentage was calculated by the mass loss. Along with the NP size, this allows a NP composition to be determined. TGA also provides some insight on the 


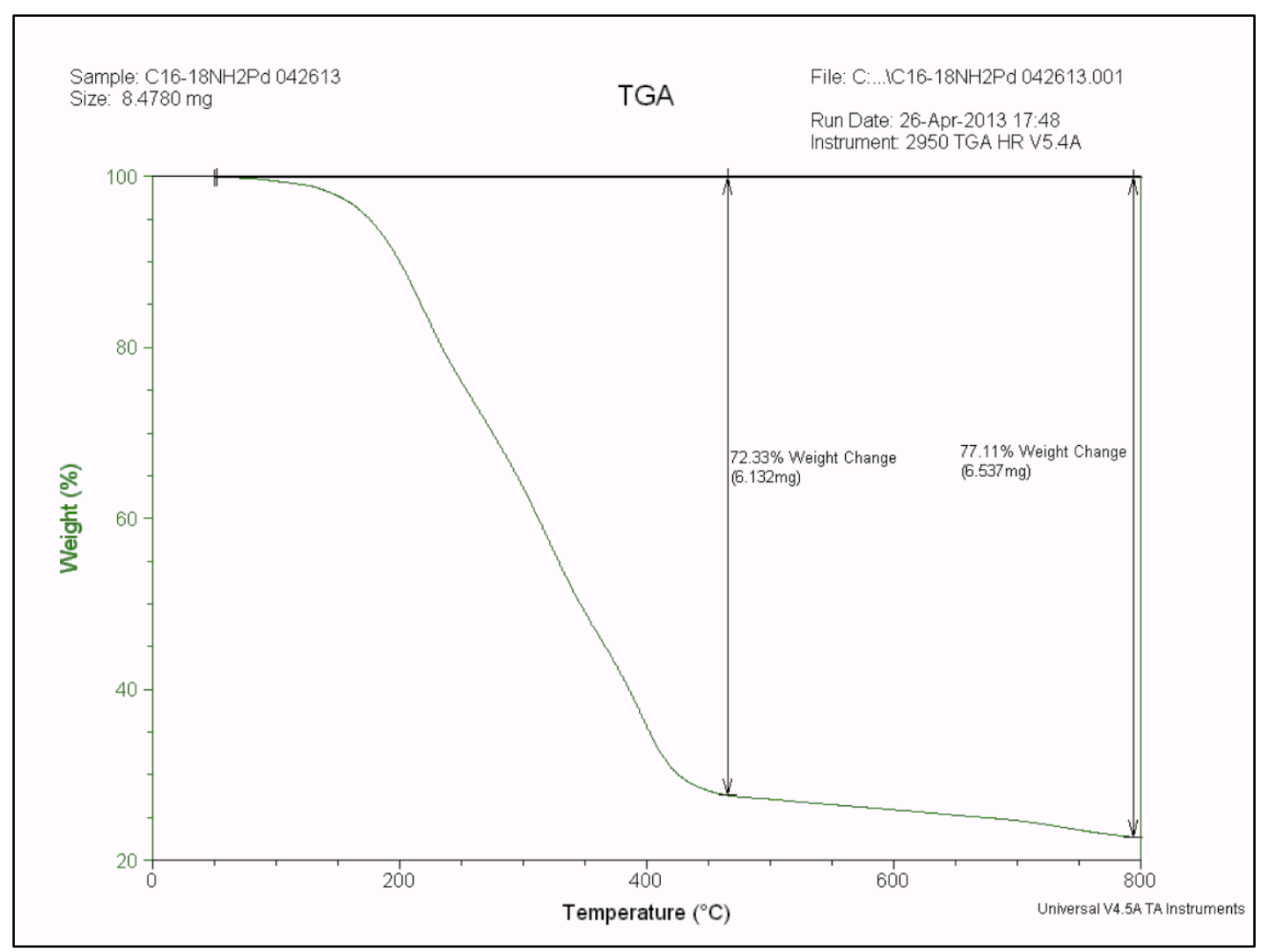

Figure 2.5. Thermogravimetric (TGA) measurement of $\mathrm{C}_{16 \mathrm{NH}_{2}} \mathrm{Pd} \mathrm{NPs}$. 


\begin{tabular}{|c|c|c|c|c|}
\hline Metal Salt & Stabilizer & $\begin{array}{c}\text { Metal: } \\
\text { Stabilizer } \\
\text { Ratio }\end{array}$ & $\begin{array}{c}\text { Reducing } \\
\text { Agent }\end{array}$ & Solvent \\
\hline $\mathrm{K}_{2} \mathrm{PdCl}_{4}$ & $\begin{array}{l}\text { Reduced-L- } \\
\text { glutathione }\end{array}$ & $1: 0.34$ & $\mathrm{NaBH}_{4}$ & Water \\
\hline $\begin{array}{c}\mathrm{K}_{2} \mathrm{PdCl}_{4} / \mathrm{HAuCl}_{4}, \\
\mathrm{~K}_{2} \mathrm{PtCl}_{4}(90: 10 \\
75: 25,50: 50)\end{array}$ & $\begin{array}{l}\text { Reduced-L- } \\
\text { glutathione }\end{array}$ & $1: 0.34$ & $\mathrm{NaBH}_{4}$ & Water \\
\hline $\begin{array}{c}\mathrm{K}_{2} \mathrm{PdCl}_{4}, \mathrm{~K}_{2} \mathrm{PdCl}_{4} / \\
\mathrm{CuSO}_{4}(2: 1) \\
\mathrm{K}_{2} \mathrm{PdCl}_{4} / \mathrm{HAuCl}_{4} \\
(1: 1)\end{array}$ & $\begin{array}{l}\text { Tri-sodium } \\
\text { citrate }\end{array}$ & $1: 1$ & $\mathrm{NaBH}_{4}$ & Water \\
\hline $\mathrm{K}_{2} \mathrm{PdCl}_{4}$ & $\begin{array}{c}\text { Alkylamine } \\
\mathrm{CnNH}_{2} \\
(\mathrm{n}=8,12,16)\end{array}$ & $1: 12$ & $\mathrm{NaBH}_{4}$ & Toluene \\
\hline
\end{tabular}

Table 2.1. Summary of the synthesis of the metal nanoparticles performed in this dissertation. 
stability of the NPs by analyzing the temperature at which the organic material desorbs from the metal, which correlates with the strength of binding between different types of stabilizers and the metal core. TGA analyses were conducted using a 2950 TGA HR V5.4A instrument with a flow rate of $100 \mathrm{~mL} / \mathrm{min}$ of $\mathrm{N}_{2}$ over a temperature range of $25-800^{\circ} \mathrm{C}$ at a heating rate of $20^{\circ} \mathrm{C} / \mathrm{min}$ using sample sizes from 7.1-12.4 mg.

\subsubsection{Nuclear Magnetic Resonance Spectroscopy ( ${ }^{1} \mathrm{H}$ NMR)}

${ }^{1} \mathrm{H}$ NMR spectroscopy gave information about the successful attachment of the ligands onto the metal core of synthesized NPs and confirms the removal of nonbound ligands. Since the peaks for the organic stabilizer gets broadened in NMR due to association with the metal core, purity was determined by the absence of sharp peaks in the spectrum. NMR analysis on all NPs were consistent with the successful synthesis of pure NPs. ${ }^{1}$ HNMR spectra were recorded on a $400 \mathrm{MHz}$ INOVA spectrometer with 64 scans from -2 to $18 \mathrm{ppm}$ at room temperature and pressure. A residual solvent peak at $\delta 4.79 \mathrm{ppm}$ of $\mathrm{D}_{2} \mathrm{O}$ was used as an internal reference for water-soluble NPs and solvent peak at $\delta 7.26 \mathrm{ppm}$ of $\mathrm{CDCl}_{3}$ was used as an internal reference for organic-soluble NPs.

\subsubsection{UV-Visible Spectroscopy}

UV-Visible spectroscopy data were used to determine the metallic composition of various synthesized NPs and their stability in the presence of hydrogen during each reaction cycle for different amounts of time. UV-Visible 
spectra were obtained using a Varian Cary 50 Bio UV-Visible spectrophotometer over the wavelength range of $200-900 \mathrm{~nm}$. All water solutions or toluene solutions containing NPs were placed in a quartz cuvette with an optical path length of $1 \mathrm{~cm}$ and the baselines of each spectrum was corrected using the solvent spectrum.

\subsubsection{Transmission Electron Microscopy (TEM)}

TEM imaging was used to determine the size and fine details of the morphology of synthesized NPs. Images of these NPs were recorded at different magnifications using a FEI Tecani F-20 field-emission microscope equipped with a CCD camera and operating at an accelerating voltage of $200 \mathrm{KeV}$. Samples were prepared by drop casting the diluted solution $(\sim 1 \mathrm{mg} / \mathrm{mL})$ of synthesized NPs in water or Toluene onto a copper grid-supported holey carbon film and allowing the solvent to evaporate at room temperature. Size distribution analysis of NP microscope images were executed with Scion Image Beta Release 2.

\subsection{Hydrogen Stability of Solution-phase glutathione and citrate-coated Pd, PdPt, PdAu and CuPd NPs}

UV-Vis spectroscopy provides information about the stability of Pd and Pdalloy NPs in solution in the presence of hydrogen for different amounts of time. The optical properties of NPs were evaluated over the range of $200-900 \mathrm{~nm}$ using a Varian-Cary 50 Bio UV-visible spectrophotometer. At room temperature, two or three drops of a $10 \mathrm{mg} / \mathrm{mL}$ water or toluene solution of Pd NPs were placed in a 1 $\mathrm{cm}$ path length quartz cuvette and diluted further with same solvent. The baseline of 
each spectrum was corrected using the spectrum of that same solvent. The absorbance spectrum of the solutions was measured following exposure to pure hydrogen bubbling through the solution at a flow rate of $19.9 \pm 0.7 \mathrm{~mL} / \mathrm{min}$ for 0,5 , 15, 30 and 60 min. Figure 2.6. shows the set-up used for $\mathrm{H}_{2}$ stability of solution of $\mathrm{Pd}$ and Pd-alloy NPs.

\subsection{Catalysis with glutathione-coated and citrate-coated Pd and Pd-alloy NPs}

The catalysis experiments of allyl alcohol to either hydrogenated and/or isomerized product with glutathione-coated Pd and Pd-alloy NPs were performed by dissolving $6.0 \mathrm{mg}$ of either Pd or Pd alloy nanoparticles in $2 \mathrm{~mL}$ of nanopure water in a $10 \mathrm{~mL}$ glass vial capped with a septum with an outlet for spent hydrogen gas passed through a glass pipette. Then, $2 \mathrm{~mL}$ of organic solvent [Chlorobenzene (Clben), Dichloromethane (DCM) or Ethylacetate (EtOAc)] and $200 \mu \mathrm{L}$ of allyl alcohol were added, forming a biphasic reaction solution mixture as shown in Figure 2.7. The reaction mixture was then stirred at $500-600 \mathrm{rpm}$ at room temperature and under atmospheric pressure. Hydrogen gas was purged at a given flow rate $(8.0 \pm 0.2,19.9 \pm 0.7$ or $39.9 \pm 0.3 \mathrm{~mL} / \mathrm{min})$ through a glass pipette for the duration of the experiment and the total volume was kept constant by adding organic solvent to the reaction vial when evaporation occurred. $20 \mu \mathrm{L}$ aliquots were removed from both aqueous and organic phases at particular intervals of time. The NPs in the aqueous phase aliquot were precipitated by adding THF solvent in 2-fold excess (40 $\mu \mathrm{L})$ and centrifuged at $4000 \mathrm{rpm}$ for $15 \mathrm{~min}$ in a small eppendrof vial. The progress of the reaction was monitored by GC-FID. GC chromatography data were recorded 


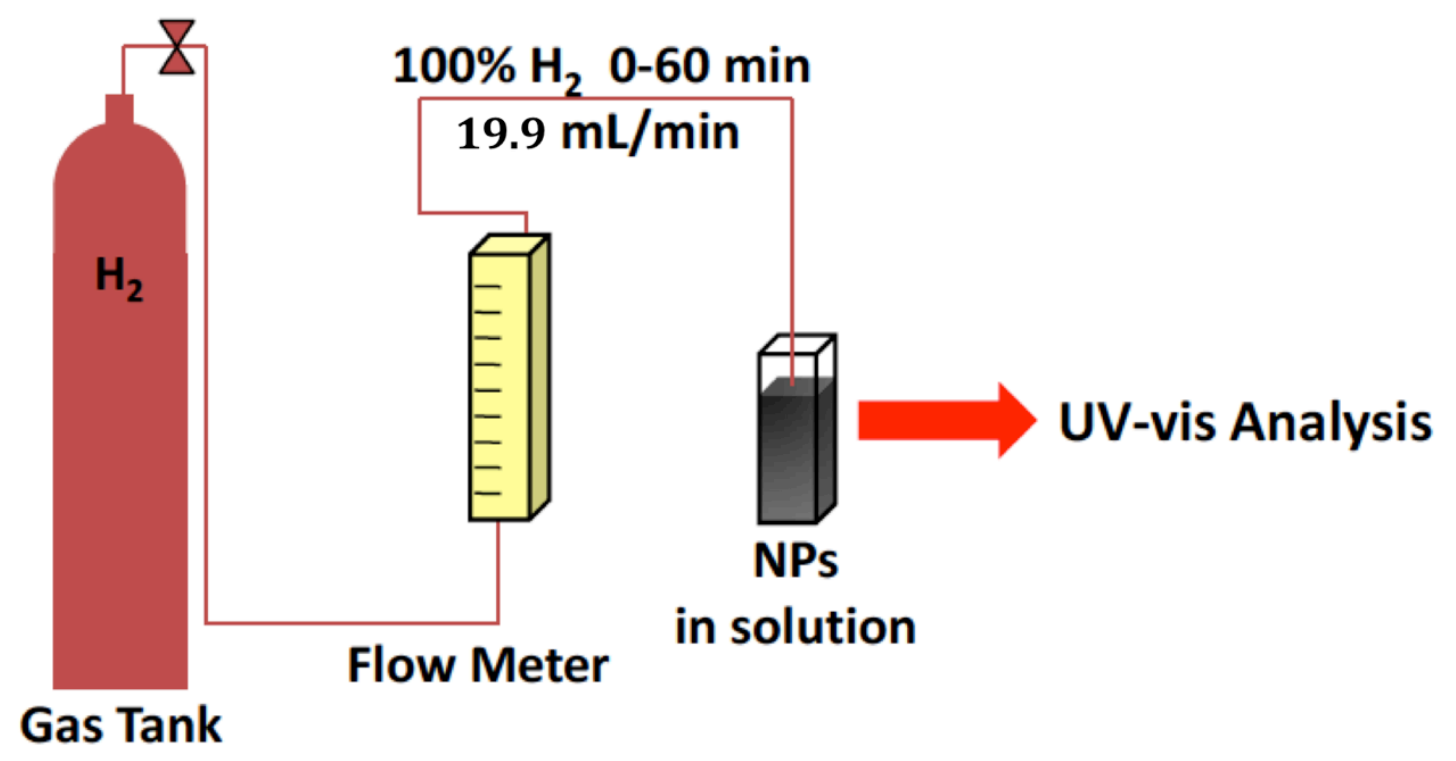

Figure 2.6. Schematic illustration of hydrogen stability set-up of NPs in solution. 
on Buck Scientific model 910 Gas Chromatograph equipped with a 1/8 ${ }^{\text {th }}$ inch packed column (10\% Carbowax 20M on silica 80/100 mesh, 6 foot) using a flame ionization detector (FID) and helium as the carrier gas. The method developed for GC includes temperatures from (a) $80^{\circ} \mathrm{C}$ to $80^{\circ} \mathrm{C}$ by holding it for $2 \mathrm{~min}$ (b) $80^{\circ} \mathrm{C}$ to $135^{\circ} \mathrm{C}$ with a ramp at $25^{\circ} \mathrm{C} / \mathrm{min}$ (c) $135^{\circ} \mathrm{C}$ to $135^{\circ} \mathrm{C}$ by holding it for $1 \mathrm{~min}$. The pressure was varied from (a) 12 psi helium for 3 min (b) 14 psi helium for 2 min.

The catalytic reaction of $\alpha$, $\beta$-unsaturated alcohols (allyl alcohol, 1-penten-3ol, 1-hepten-3-ol or 2-methyl-3-buten-2-ol) to hydrogenated and/or isomerized product with citrate-coated Pd and Pd-alloy NPs were performed in a biphasic solvent mixture by adding $2 \mathrm{~mL}$ of dichloromethane in $2 \mathrm{~mL}$ of already synthesized aqueous Pd NPs solution containing $0.15 \mathrm{mg}$ of calculated citrate-Pd or Pd-alloy NPs under hydrogen gas purging at a flow rate of $19.9 \pm 0.7 \mathrm{~mL} / \mathrm{min} .20 \mu \mathrm{L}$ aliquots were removed from both aqueous and organic phases at particular intervals of time. The reaction mixture was stirred vigorously at $600-700 \mathrm{rpm}$ at room temperature and atmospheric pressure.

\subsubsection{Gas Chromatography-Flame Ionization Detector (GC-FID)}

The progress of the catalytic reaction was followed by Gas Chromatography of samples before exposure to hydrogen and after addition of NP catalyst and exposure to hydrogen for different timings. The catalytic reaction was monitored using response factors that were determined with a standard mixture containing 10 $\mathrm{mM}$ of each reactant and products. Four structurally related allyl alcohols were studied: 2-propen-1-ol, 1-penten-3-ol, 1-hepten-3-ol and 2-methyl-3-buten-2-ol in a 

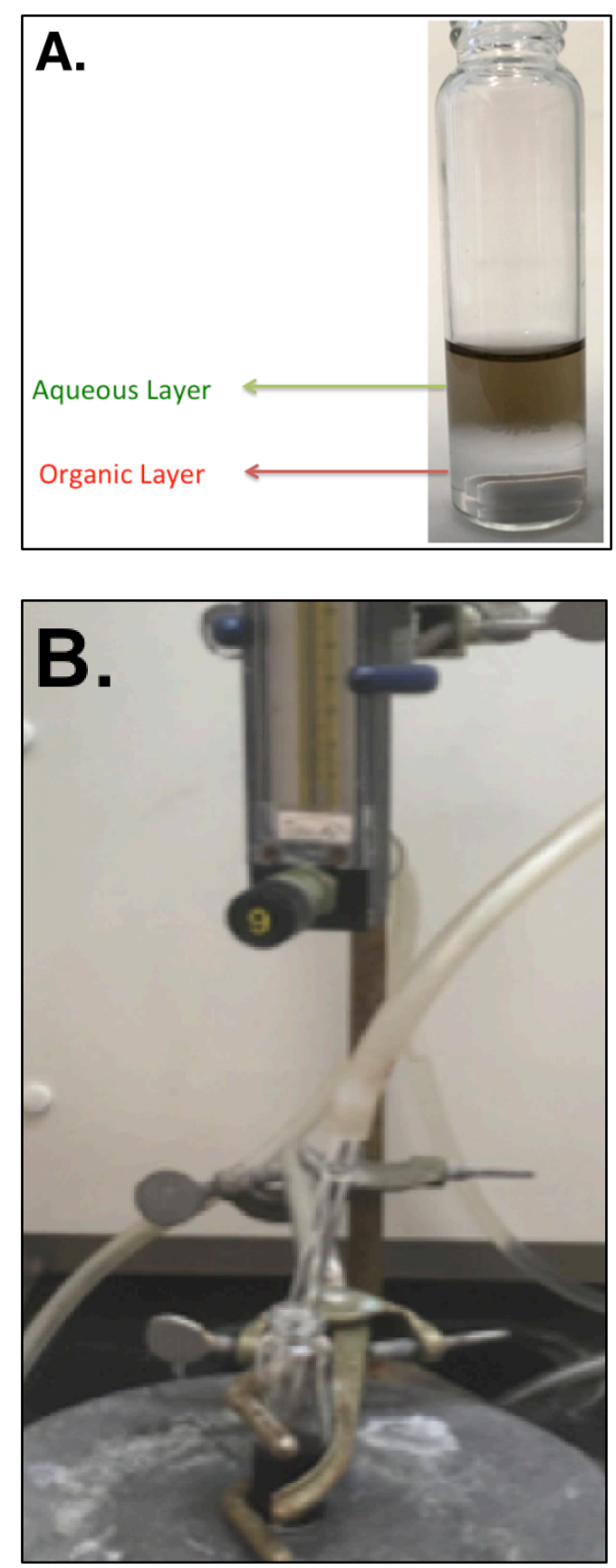

Figure 2.7. Biphasic reaction mixture involving aqueous and organic phases (A). And complete set-up of the catalytic reaction involving passing hydrogen gas through the glass pipette into the reaction mixture (B). 
biphasic solvent mixture which give possibly the following hydrogenation and/or isomerization products in the presence of Pd or Pd-alloy NP catalysts and hydrogen: propanol/propanal, 3-pentanol/3-pentanone, 3-heptanol/3-heptanone and 2methyl-2-butanol, respectively. Figure 2.8. shows an example of gas chromatogram of a standard solution containing 2-propen-1-ol as reactant with its corresponding products and retention times for organic phase and aqueous phase in Frames A and B, respectively.

\subsection{Catalysis studies for the $\mathrm{C}-\mathrm{C}$ cross coupling Suzuki-Miyaura reaction}

Pd catalyzed C-C cross coupling of alkyl halides and aryl boronic acids, known as Suzuki-Miyaura reaction, has been widely used since the last three decades for synthesizing unsymmetrical biaryls. Studying Suzuki-Miyaura reaction using 4-Iodoanisole and Phenylboronic acid is useful as a model reaction since 4iodoanisole is solid and non-volatile, hence can be easily detected on TLC and in GCMS. Also, the $-\mathrm{OCH}_{3}$ group is the activating group in electrophilic-aromatic substitution reactions. Also, we can see any homo-coupled biphenyl product if forming as it will be different from 4-methoxyphenyl benzene product.

The procedure for the Suzuki reaction involves mixing of arylhalide (4Iodoanisole, 0.500 gms, 1.0 mole equi.), arylboronic acid (Phenylboronic acid, 0.338 gms, 1.3 mole equi.) and Pd NPs (0.015 gms, 1.5 mole \%) as the catalyst in a toluene:water (10 mL: $1 \mathrm{~mL})$ biphasic mixture, where the base $\left(\mathrm{K}_{2} \mathrm{CO}_{3}, 1.181 \mathrm{gms}\right.$, 4.0 mole equi.) is dissolved in a minimal amount of water (10\% aqueous solution) and the reaction mixture is heated at a particular temperature. 

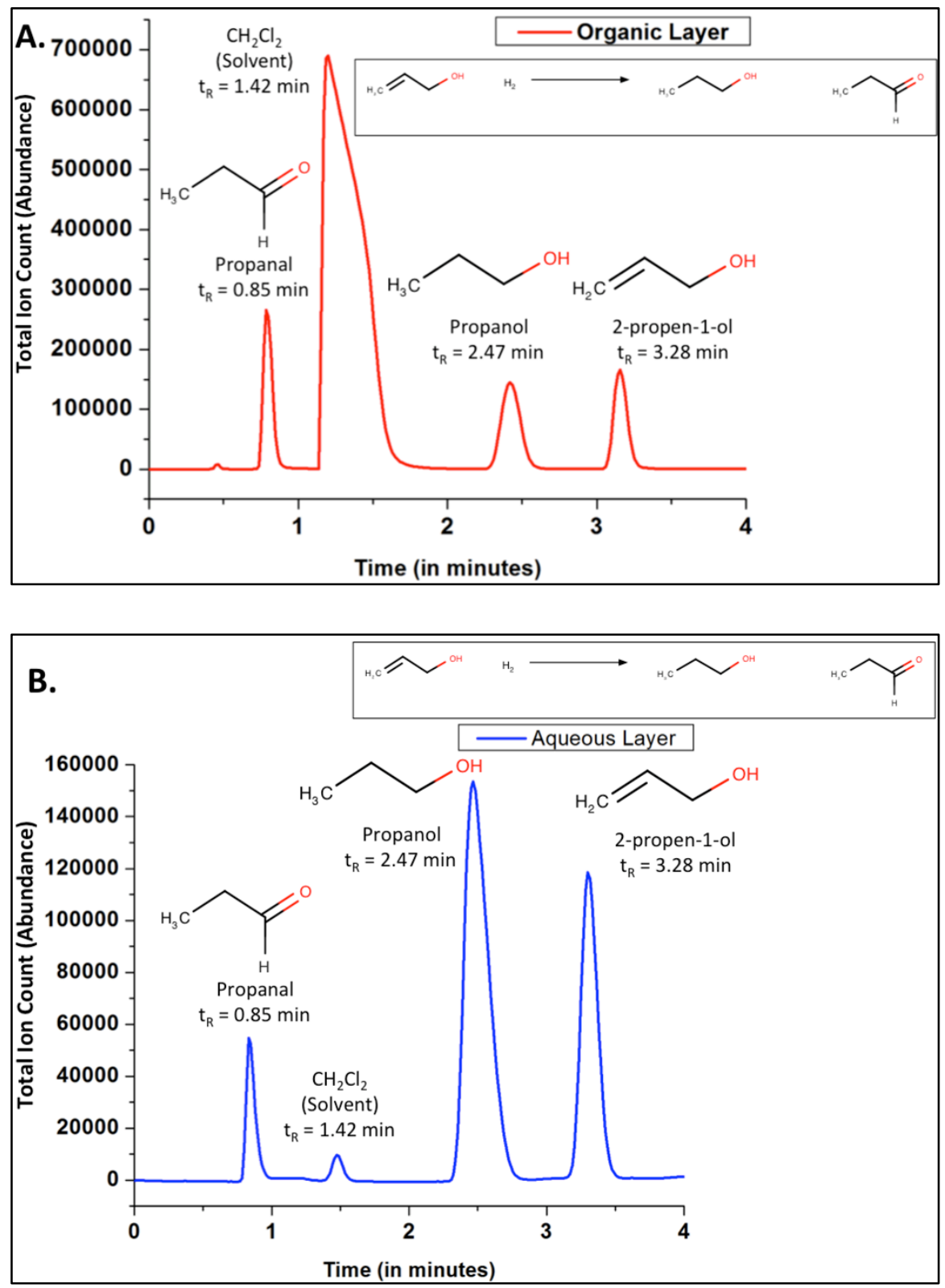

Figure 2.8. Gas chromatogram of a standard solution containing $10 \mathrm{mM}$ of 2 propen-1-ol (reactant), propanal and 2-propen-1-ol as products and their retention times for both organic phase (Dichloromethane) (A) and aqueous phase (B). Integration was performed using instrument software. Injection volume $=1 \mu \mathrm{L}$, initial temperature $=80^{\circ} \mathrm{C}$, final temperature $=135^{\circ} \mathrm{C}, \mathrm{ramp}=$ $25^{\circ} \mathrm{C}$, pressure $=14 \mathrm{PSI}$, Detector $=$ FID . 
The progress of the catalytic reaction was followed by Thin-Layer Chromatography (TLC) and Gas Chromatography-Mass spectrometry for samples before and after addition of NP catalyst for different timings. GC chromatography data were recorded on a GCxGC-TOF MS platform using a LECO Pegasus III time-offlight mass spectrometer equipped with a Gerstel MPS2 auto-sampler. The Pegasus 4D GCxGC-TOF MS instrument has an Agilent 6890 gas chromatograph featuring a LECO two-stage cryogenic modulator and a secondary oven. Data acquisition and processing were performed by Chroma TOF (LECO) software. The method developed for $\mathrm{GC}$ includes temperatures from (a) $130^{\circ} \mathrm{C}$ to $130^{\circ} \mathrm{C}$, holding for $2 \mathrm{~min}$ (b) $130^{\circ} \mathrm{C}$ to $200^{\circ} \mathrm{C}$, ramp at $25^{\circ} \mathrm{C} / \mathrm{min}$, and (c) $200^{\circ} \mathrm{C}$ to $200^{\circ} \mathrm{C}$, holding for $1 \mathrm{~min}$. The pressure remains 20 psi helium for 5 min. As shown in Figure 2.9, the Gas chromatogram of a Suzuki reaction involving 4-iodoanisole and phenylboronic acid fused to form as triphenylboroxane as reactants and 4-methoxyphenyl benzene as product at their respective retention times. 


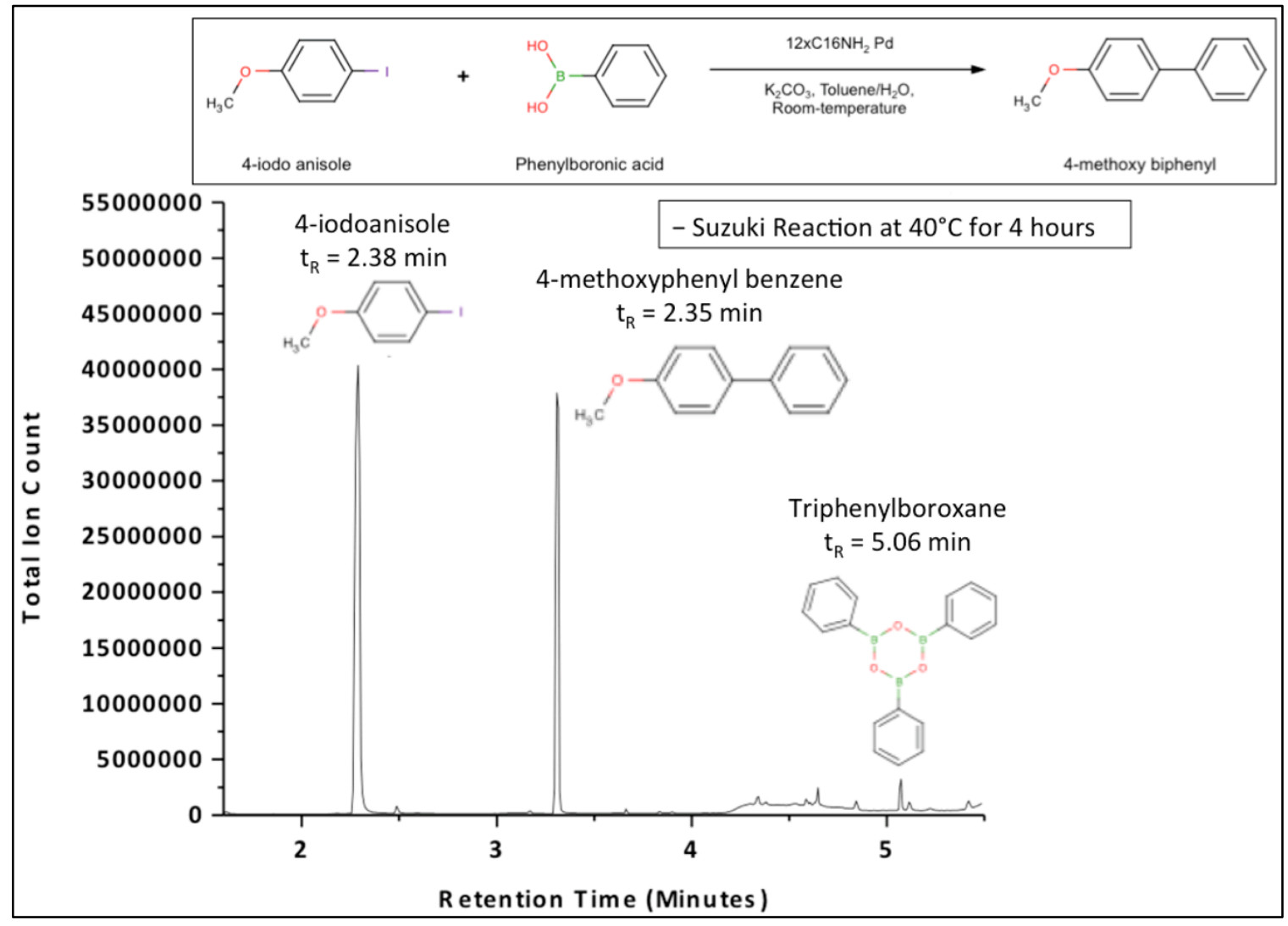

Figure 2.9. Gas chromatogram of a model Suzuki reaction containing 4iodoanisole (0.500 gms, 1.0 mole equi.), phenylboronic acid (0.338 gms, 1.3 mole equi.), $12 \times \mathrm{xC} 12 \mathrm{NH}_{2}$ Pd NPs (0.015 gms, 1.5 mole \%), Potassium carbonate (1.181 gms, 4.0 mole equi.), Toluene (10 mL), Water ( $1 \mathrm{~mL}, 10 \%$ aqueous solution). Integration was performed using instrument software. Injection volume $=1 \mu \mathrm{L}$, initial temperature $=130^{\circ} \mathrm{C}$, final temperature $=$ $200^{\circ} \mathrm{C}$, ramp $=25^{\circ} \mathrm{C}$, pressure $=20 \mathrm{PSI}$, Detector $=\mathrm{MS}$. 


\section{CHAPTER III}

HYDROGEN AND THERMAL STABILITY OF SOLUTION-PHASE Pd, PdPt AND PdAu NANOPARTICLES (NPS)

\subsection{INTRODUCTION}

Since, hydrogen is going to be one of the reactants in the hydrogenation/ isomerization reactions studied and high temperature used in the Suzuki Coupling reactions, it is very important for the metal nanoparticles to be stable under these conditions and reactive. Here, we study the stability of glutathione-coated Pd NPs, citrate-coated Pd and Pd alloy NPs in hydrogen. We compare the NPs synthesized under inert and ambient conditions and study the effect of the substrates by UV-Vis spectroscopy. We also studied the stability of variable chain-length alkylaminecoated Pd and Pd alloy NPs at high temperature without and with base and substrates. These studies provide interesting knowledge about the stability of NPs under various catalytic conditions, which is useful for the catalysis studies described in Chapters IV and V.

\subsection{RESULTS AND DISCUSSION}




\subsubsection{Hydrogen stability of glutathione-coated Pd NPs}

UV-Vis spectroscopy provides information about the stability of Pd NPs in solution in the presence of hydrogen for different amounts of time. Figure 3.1. shows the UV-Vis spectra of aqueous solutions of Pd-Glu (Inert) and Pd-Glu (Oxy) NPs from 200-900 $\mathrm{nm}$ as a function of exposure time to pure hydrogen bubbling at $19.9 \pm 0.7 \mathrm{~mL} / \mathrm{min}$ through these solutions for $0,5,30,60$ and 120 minutes. Due to the strong binding of glutathione ligands with Pd NPs through the thiolate group, the NPs showed a very similar spectrum for at least 2 hours, confirming that they were highly stable against hydrogen-induced aggregation. The UV-Vis spectra of these small glutathione-coated Pd NPs shows an exponential decay in absorbance with an increase in wavelength and lack any surface plasmon resonance bands since Pd has no surface plasmon band in this region. The inset for both Frame (A) and (B) shows the Pd NP solution in water before exposure to hydrogen at 0 minute and after exposure to hydrogen for 120 minutes. The color of the solutions remained visibly the same, also confirming the high stability of the Pd NP solutions against hydrogen-induced aggregation.

The same trend was observed for all Pd-alloy NPs with $\mathrm{Au}$ and Pt with different ratios synthesized under both inert and oxygen atmosphere confirming that glutathione ligands provide high stability in the presence of hydrogen. These NPs were remained highly soluble in water and exhibited an exponential decrease in absorbance during hydrogen exposure for 120 minutes. 

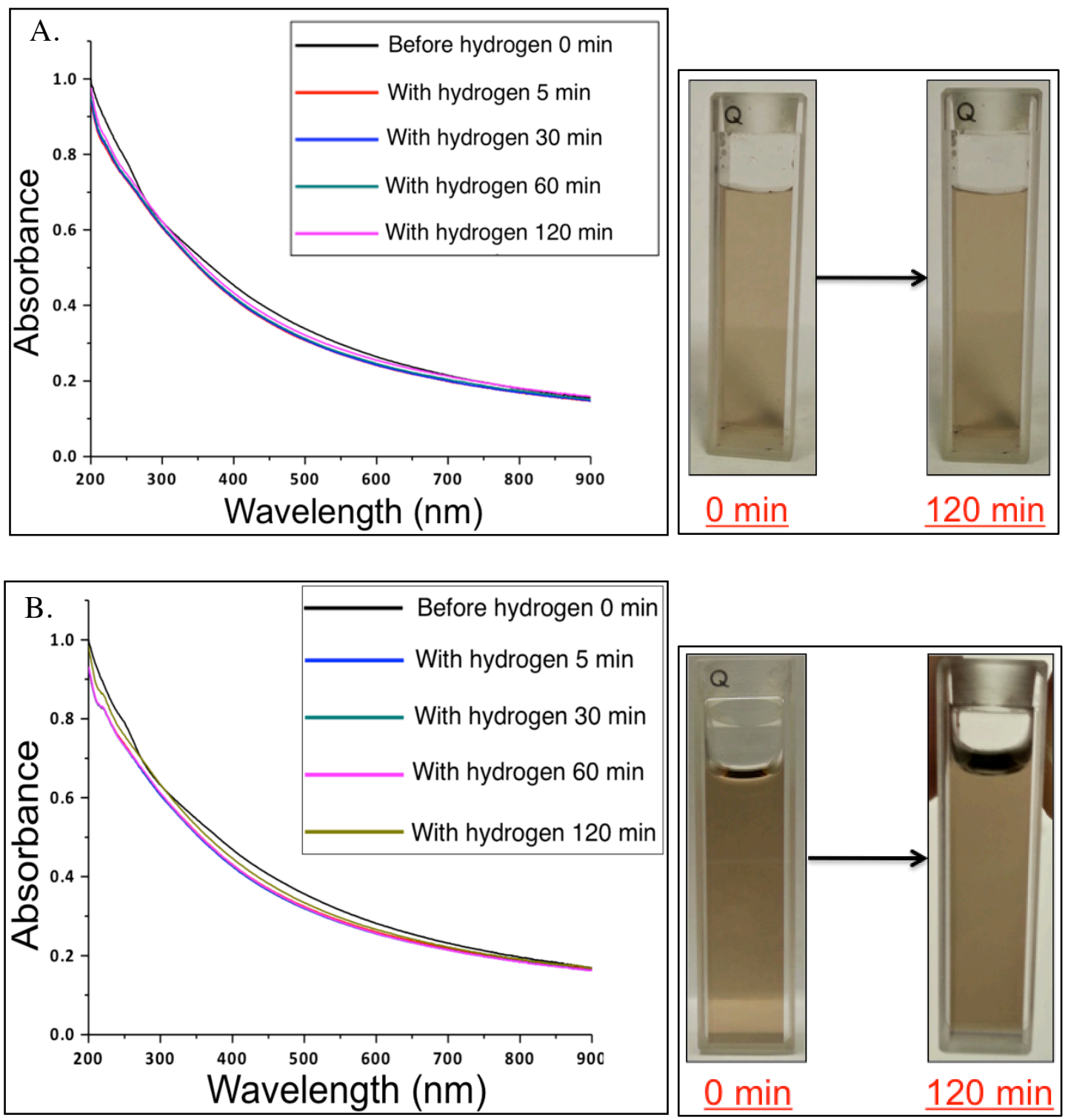

Figure 3.1. UV-Vis spectra of (A) Pd-Glu (Oxy) nd (B) Pd-Glu (Inert) NPs for exposure time $0-120 \mathrm{~min}$ to $100 \%$ hydrogen bubbling through the aqueous solutions at $19.9 \pm 0.7 \mathrm{~mL} / \mathrm{min}$. The insets show pictures of solutions before exposure to hydrogen at 0 minute and after exposure to hydrogen at 120 minutes. 
In order to understand the behavior of Pd NPs in the presence of substrate only without having any effects of the organic solvent generally present in the biphasic solvent system, we checked the hydrogen stability of Pd-Glu (Inert) NPs at different intervals of time under monophasic aqueous solution in the presence of allyl alcohol with $100 \%$ hydrogen at $19.9 \pm 0.7 \mathrm{~mL} / \mathrm{min}$ flow rate for $0,30,60,90$, 120 and 150 minutes using UV-Vis spectroscopy as shown in Figure 3.2. The spectra looked very similar and the Pd-Glu (Inert) NP aqueous solution showed a similar color after 150 minutes as before exposure to hydrogen, showing high stability against hydrogen-induced aggregation.

We also studied the stability of Pd-Glu (Inert) NPs and Pd-Glu (Oxy) NPs against hydrogen-induced aggregation under the biphasic condition of chlorobenzene and nanopure water in the presence of allyl alcohol with $100 \%$ hydrogen at $19.9 \pm 0.7 \mathrm{~mL} / \mathrm{min}$ flow rate for $0,5,15,30,60,120$ and 240 minutes using UV-Vis spectroscopy as shown in Figure 3.3. While the absorbance of Pd-Glu (inert) NPs remained almost the same displaying a stable absorbance as shown in Frame (A), the absorbance of Pd-Glu (Oxy) NPs decreased gradually with time, showing some aggregation and changes to the NPs in the presence of hydrogen as shown in Frame (B). Also, higher concentration of Pd(II) peak appears for Pd-Glu (Oxy) NPs than Pd-Glu (Inert) NPs at $\sim 250 \mathrm{~nm}$ suggesting the oxidation of Pd atoms during the reaction resulting in less stability of the NPs. Thus, UV-Vis spectra showed Pd-Glu (Inert) to be comparatively more stable than Pd-Glu (Oxy) NPs against $\mathrm{H}_{2}$-induced aggregation. 


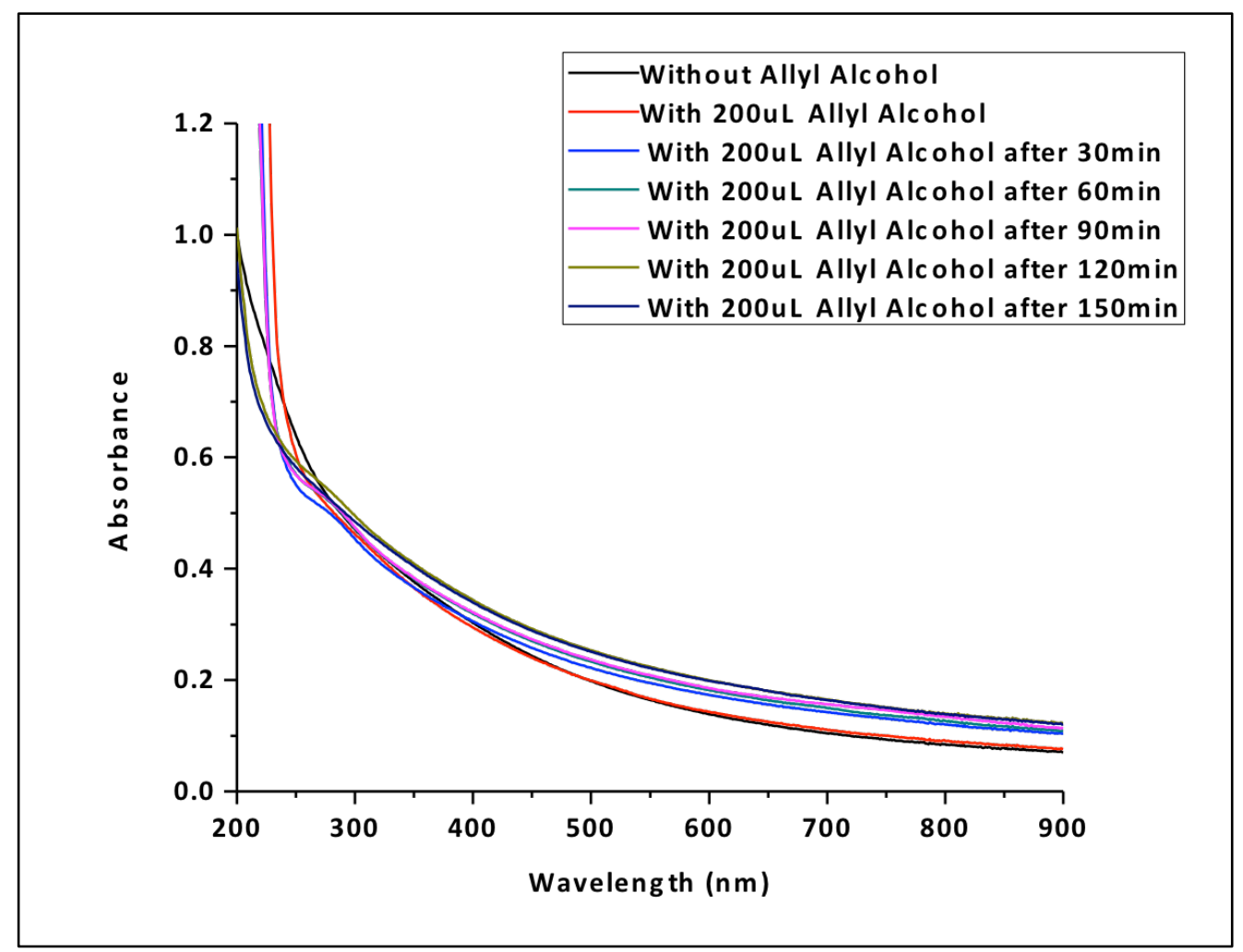

Figure 3.2. UV-Vis spectra of aqueous Pd-Glu (Inert) NP solution against hydrogen-induced aggregation under monophasic conditions in the presence of allyl alcohol at $19.9 \pm 0.7 \mathrm{~mL} / \mathrm{min}$ hydrogen flow rate for $0,30,60,90,120$ and 150 minutes. 

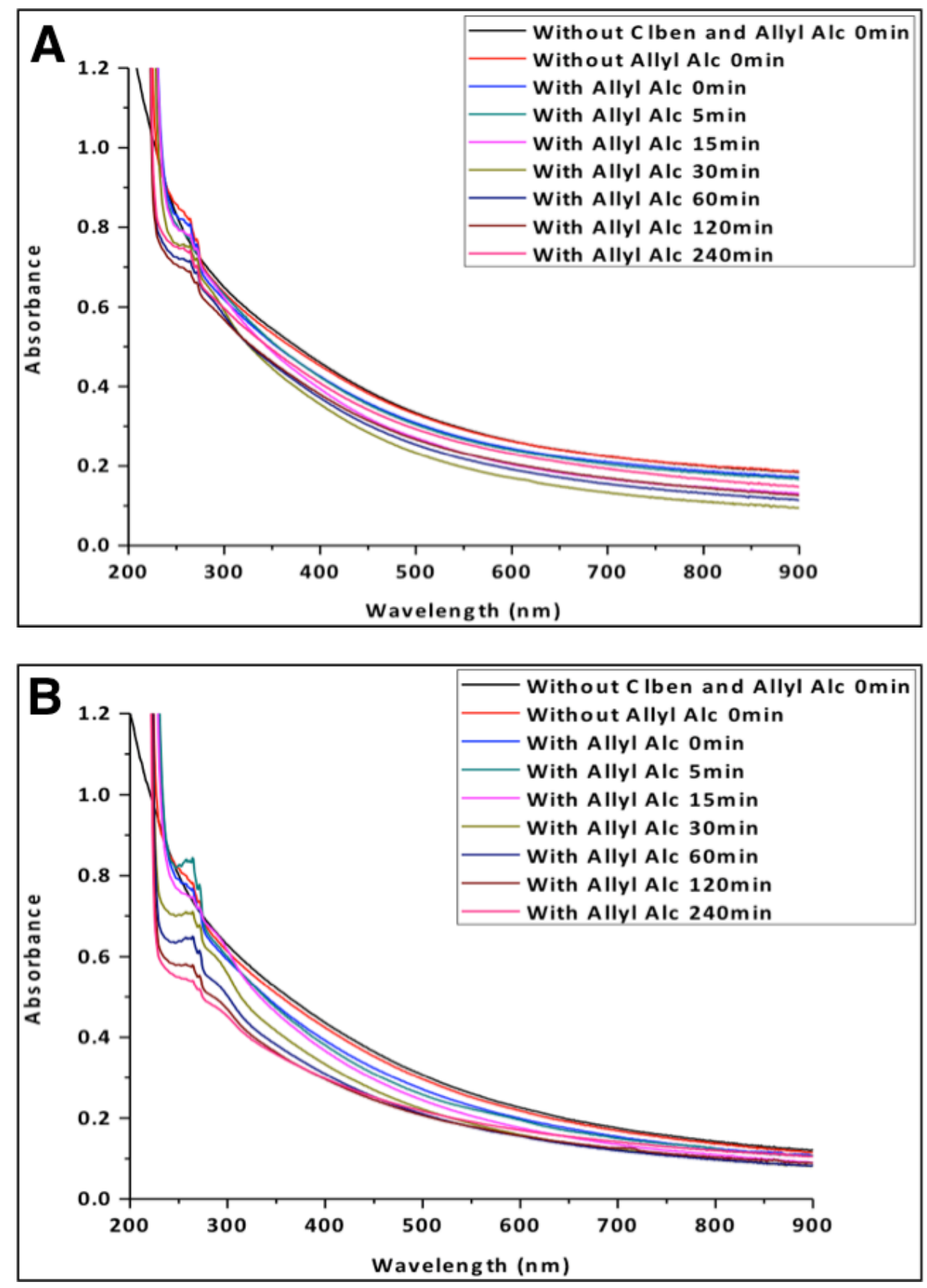

Figure 3.3. UV-Vis spectra of Pd-Glu (Inert) NPs (A) and Pd-Glu (Oxygen) NPs (B) against hydrogen-induced aggregation under biphasic conditions of chlorobenzene and nanopure water in the presence of allyl alcohol at $19.9 \pm 0.7 \mathrm{~mL} / \mathrm{min}$ hydrogen flow rate for $0,5,15,30,60,120,240$ minutes. 
We also studied the stability of (75:25) Pd:Au-Glu (Inert) alloy NPs under the biphasic conditions of nanopure water and chlorobenzene in the absence and presence of allyl alcohol at $19.9 \mathrm{~mL} \pm 0.7 \mathrm{~mL} / \mathrm{min}$ hydrogen flow rate for $0,5,15,30$, 60, 120 and 240 minutes using UV-Vis spectroscopy as shown in Figure 3.4. The (75:25) Pd:Au-Glu (Inert) NPs in the absence of allyl alcohol showed a small absorbance decrease and a peak near $250 \mathrm{~nm}$, showing the formation of some $\mathrm{Pd}(\mathrm{II})$ species, but the NPs showed good stability overall. In the presence of allyl alcohol, the (75:25) Pd:Au-Glu (Inert) NPs showed a relatively higher concentration of Pd(II) species which shows that the substrate has some effect on the Pd surface by interacting with the Pd atoms, causing the formation of $\mathrm{Pd}(\mathrm{II})$ species.

In order to check whether glutathione-coated Pd NPs are stable for a long period of time in aqueous solution, we studied the stability of Pd-Glu (Inert) NPs under aqueous solution at the beginning and after keeping it as an aqueous solution for 60 days, and then monitored its stability to $19.9 \mathrm{~mL} \pm 0.7 \mathrm{~mL} / \mathrm{min}$ of hydrogen flow rate for $0,5,15,30,60$ and 120 minutes using UV-Vis spectroscopy as shown in Figure 3.5. The Pd-Glu (Inert) NPs showed high stability even after keeping it in aqueous solution for 60 days by showing a similar UV-vis spectrum throughout the hydrogen exposure. 

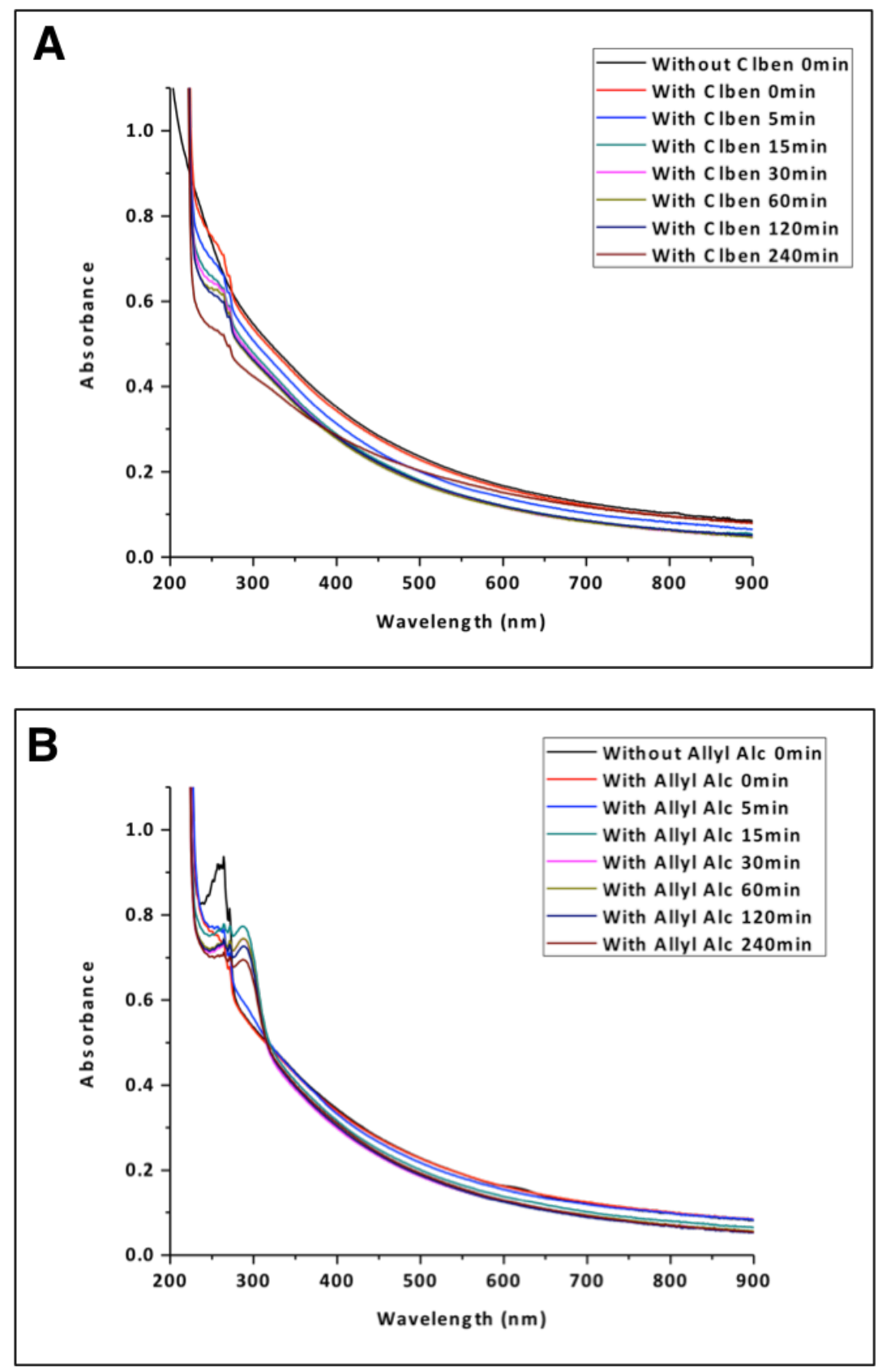

Figure 3.4. UV-Vis spectra of (75:25) Pd:Au-Glu (inert) NPs under biphasic conditions of chlorobenzene and nanopure water (A) in the absence and (B) in the presence of allyl alcohol at $19.9 \pm 0.7 \mathrm{~mL} / \mathrm{min}$ hydrogen flow rate for 0 , 5, 15, 30, 60, 120 and 240 minutes. 

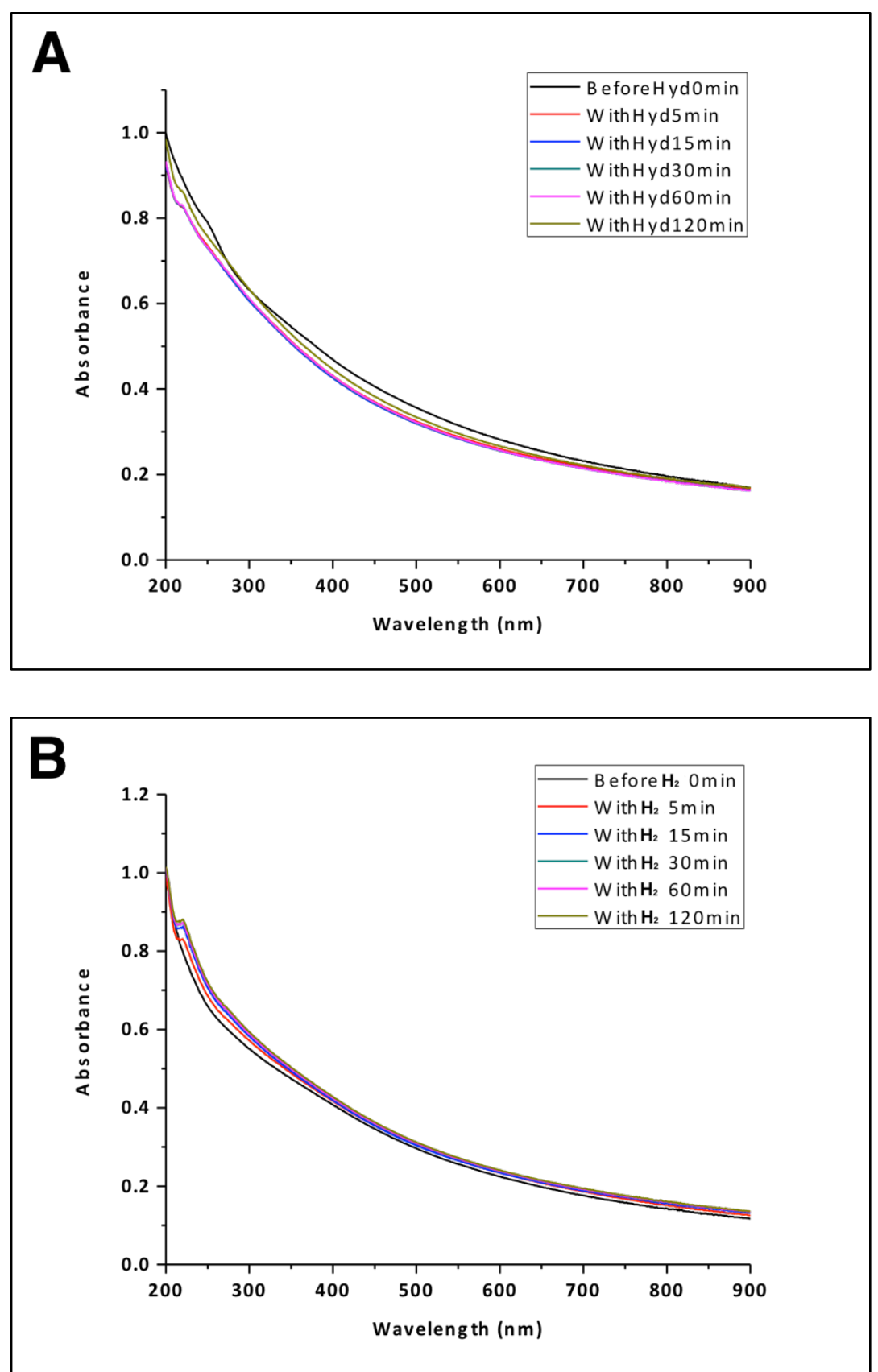

Figure 3.5. UV-Vis spectra of (A) freshly prepared Pd-Glu (Inert) NPs and (B) Pd-Glu (Inert) NPs in aqueous solution for 60 days in the presence of hydrogen flow at $19.9 \mathrm{~mL} \pm 0.7 \mathrm{~mL} / \mathrm{min}$ for $0,5,15,30,60$ and 120 minutes. 


\subsubsection{Hydrogen stability of citrate-coated Pd NPs}

We also determined the stability of the aqueous Pd-citrate NPs solution by monitoring the UV-Vis spectra from $200-900 \mathrm{~nm}$ as a function of exposure time for different amounts of time to pure hydrogen at $19.9 \pm 0.7 \mathrm{~mL} / \mathrm{min}$ flow rate bubbling through the solution. Since the citrate ligands are physisorbed on the Pd core surface, ${ }^{179}$ most of the Pd surface is available for hydrogen to get adsorbed to form palladium hydride species. In general, the presence of hydrogen with citrate-capped Pd NPs has resulted in the formation of Pd (II) species that probably leached out from the palladium core as seen by a peak at $\sim 250 \mathrm{~nm}$ in the UV-vis spectra since citrate ligands are loosely bound to the core surface of the nanoparticles. The NPs showed a very similar spectrum with exponential decay in absorbance with an increase in wavelength for at least 60 minutes, confirming that they were stable against hydrogen-induced aggregation.

\subsubsection{Stability of Pd-citrate NPs without substrate}

We determined the stability of the aqueous Pd-citrate NPs solution without the presence of substrate by monitoring the UV-vis spectra ranging from 200-900 $\mathrm{nm}$ as a function of exposure time for 60 minutes to pure hydrogen at $19.9 \pm 0.7$ $\mathrm{mL} / \mathrm{min}$ flow rate bubbling through the solution as shown in Figure 3.6. The presence of hydrogen resulted in the formation of Pd(II) species as seen by a peak at $\sim 250 \mathrm{~nm}$ in the UV-Vis spectra. The NPs showed a very similar spectrum with exponential decay in absorbance with an increase in wavelength for at least $60 \mathrm{~min}$, 


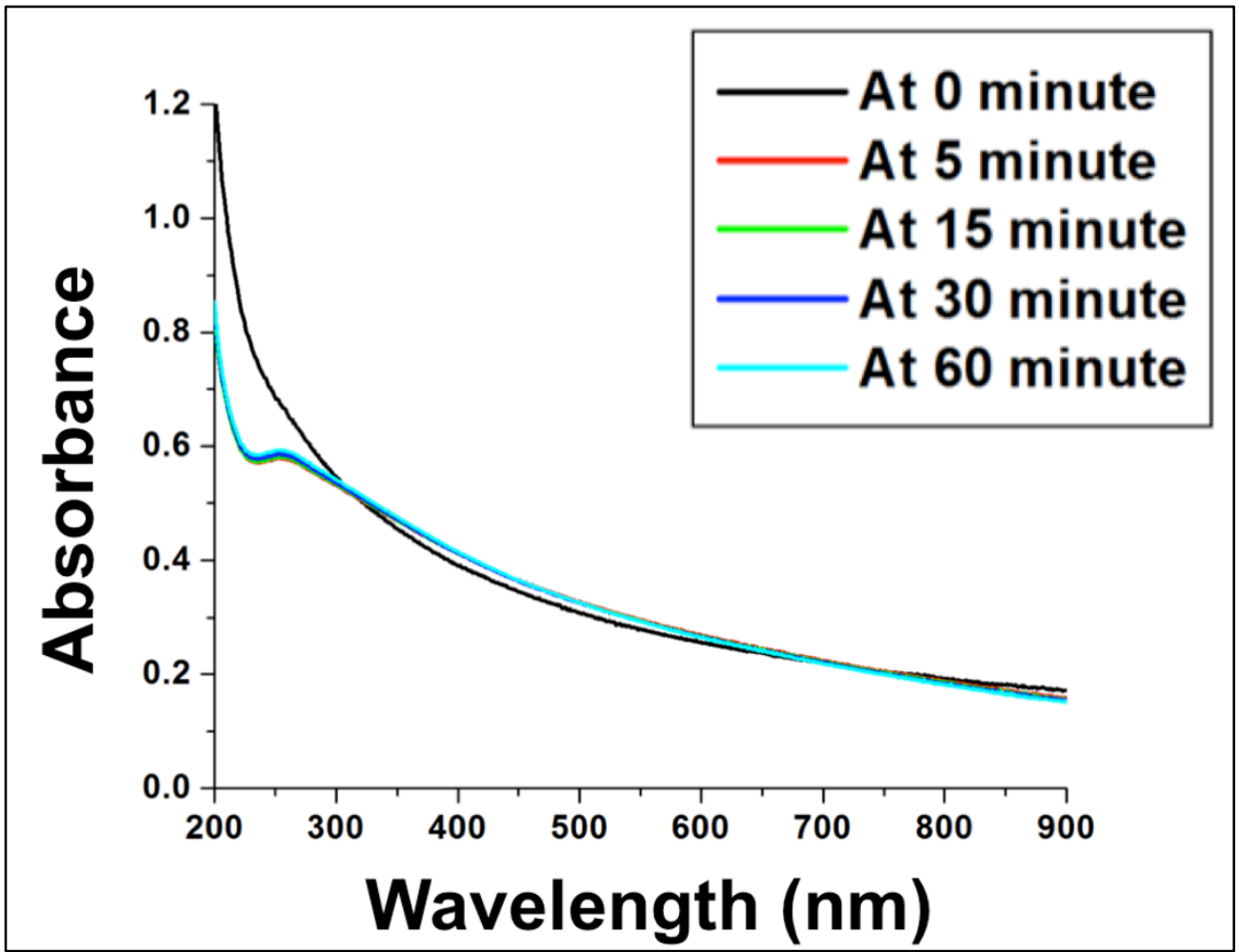

Figure 3.6. UV-Vis spectra of Pd-citrate NPs against hydrogen-induced aggregation in nanopure water in the absence of substrate at $19.9 \pm 0.7$ $\mathrm{mL} / \mathrm{min}$ hydrogen flow rate for $0,5,15,30,60$ minutes. 
confirming that they were stable against hydrogen-induced aggregation, even though Pd(II) formed.

\subsubsection{Stability of Pd-citrate NPs in presence of substrate}

We also studied the stability of Pd-citrate NPs against hydrogen-induced aggregation under the biphasic condition of dichloromethane and nanopure water in the presence of $0.74 \times 10^{-3}$ moles of $\alpha, \beta$-unsaturated alcohols (allyl alcohol, 1penten-3-ol, 1-hepten-3-ol or 2-methyl-3-buten-2-ol) at 19.9 $\pm 0.7 \mathrm{~mL} / \mathrm{min} \mathrm{H}_{2}$ flow rate for 0, 5, 15, 30, 60 minutes as shown in Figures 3.7. The UV-vis spectra for Pdcitrate NPs in the presence of allyl alcohol shows low stability and the presence of high concentration of Pd (II) species formed within the first 5 minutes of the reaction. Pd-citrate NPs in the presence of 1-hepten-3-ol showed almost a similar trend of low stability but with less formation of Pd (II) species as confirmed by a comparatively smaller peak height at $250 \mathrm{~nm}$. Pd-citrate NPs in the presence of 1penten-3-ol showed a comparatively higher stability with a very similar spectrum for the first 15 minutes and less formation of Pd (II) species. Also, the stability of Pdcitrate NPs in the presence of 2-methyl-3-buten-2-ol, having the same number of carbon atoms as 1-penten-3-ol, showed a similar high stability and low Pd (II) species formation. 

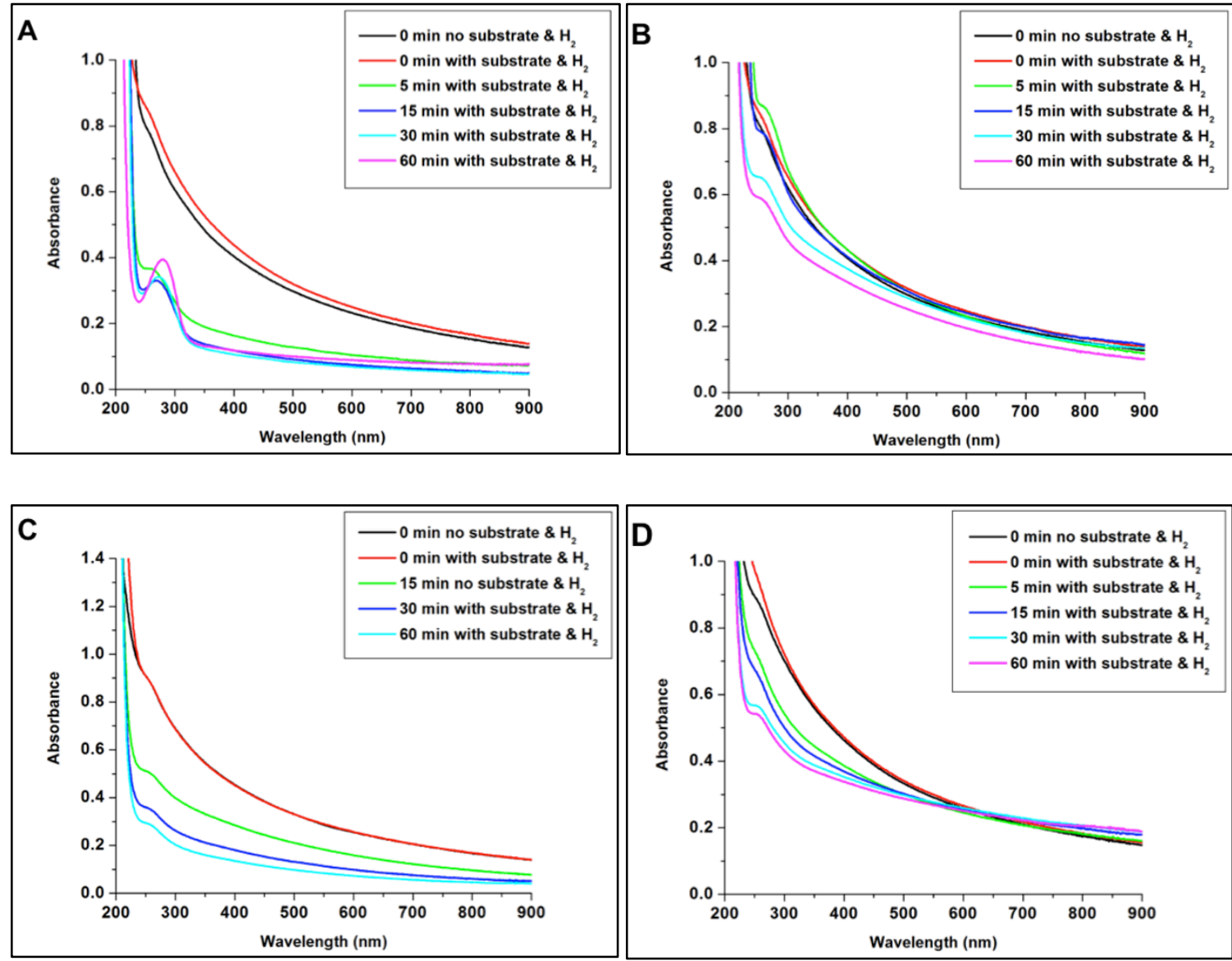

Figure 3.7. UV-Vis spectra of Pd-citrate NPs against hydrogen-induced aggregation under biphasic conditions of $2 \mathrm{~mL}$ dichloromethane and $2 \mathrm{~mL}$ nanopure water at $19.9 \pm 0.7 \mathrm{~mL} / \mathrm{min}$ hydrogen flow rate for $0,5,15,30,60$ minutes in the presence of $0.74 \times 10^{-3}$ moles of (A) allyl alcohol, (B) 1-penten3-ol, (C) 1-hepten-3-ol, and (D) 2-methyl-3-buten-2-ol. 


\subsubsection{Thermal Stability of Solution-phase alkylamine-coated Pd NPs $\left(\mathrm{CBNH}_{2}\right.$, $\mathrm{C12NH}_{2}$ and $\left.\mathrm{C16NH}_{2} \mathrm{Pd} \mathrm{NPs}\right)$}

Since, Suzuki reaction involves heating of reaction mixture, there was need to determine the thermal stability of solutions of various chain lengths of alkylaminestabilized Pd NPs like $\mathrm{C}_{16 \mathrm{NH}_{2}} \mathrm{Pd}, \mathrm{C} 12 \mathrm{NH}_{2} \mathrm{Pd}$ and $\mathrm{C} \mathrm{NH}_{2}$ Pd NPs, which can act as potential catalysts, using UV-Vis absorbance spectrum as a function of temperature ranging for 25 to $95^{\circ} \mathrm{C}$ as shown in Figure 3.8. (A). UV-visible spectroscopy showed that the absorbance for $\mathrm{C}_{16} \mathrm{NH}_{2}$ Pd NPs remains high for all temperatures whereas absorbance for $\mathrm{C}_{12 \mathrm{NH}_{2}} \mathrm{Pd}$ and $12 \mathrm{xC} 8 \mathrm{NH}_{2} \mathrm{Pd}$ NPs decreases rapidly at high temperatures which shows temperature-induced aggregation and thus, lower stability of these NPs due to weak interaction of amines with the Pd core and shorter chain-length.

Thus, we decided to use and study $\mathrm{C}_{16} \mathrm{NH}_{2}$ Pd NPs as catalyst for the Suzuki reaction. Pd-C16 $\mathrm{NH}_{2} \mathrm{NPs}$ were again tested in toluene solution using UV-Vis spectrum as a function of different temperatures ranging for 30 to $110^{\circ} \mathrm{C}$ each heated for at least 30 minutes as shown in Frame (B). The absorbance remains high

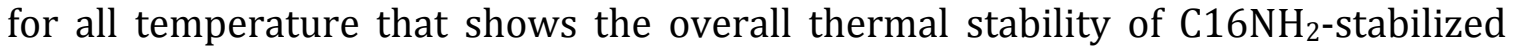
NPs due to bilayer coating of $\mathrm{C}_{16 \mathrm{NH}_{2}}$ ligands on $\mathrm{Pd}$ core ${ }^{75}$ and thus thermally suitable for use as a catalyst in the Suzuki reaction. But, when all the components of the Suzuki reaction (toluene, water, $\mathrm{K}_{2} \mathrm{CO}_{3}$, phenyl-boronic acid, 4-iodoanisole and $\mathrm{C}_{16 \mathrm{NH}_{2}} \mathrm{Pd} \mathrm{NPs}$ ) were stirred at temperatures ranging from $40^{\circ} \mathrm{C}-70^{\circ} \mathrm{C}$ each for 30 minutes, the NPs weren't stable after $60^{\circ} \mathrm{C}$ as shown in Frame (C). Further experiments are going on to add primary reactants (4-iodoanisole and pheylboronic 

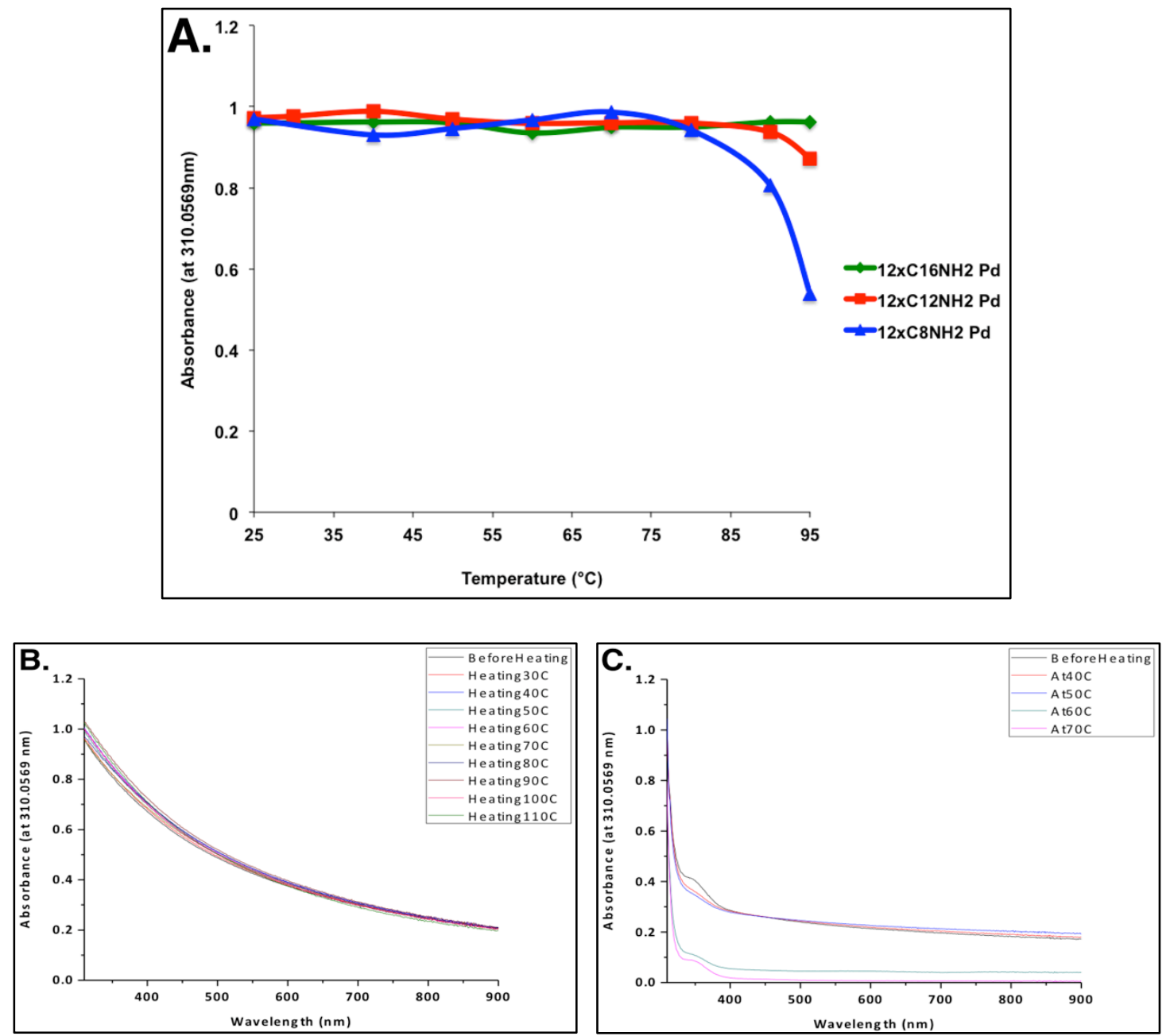

Figure 3.8. UV-Vis plots for different temperatures (A) ranging from 25 to

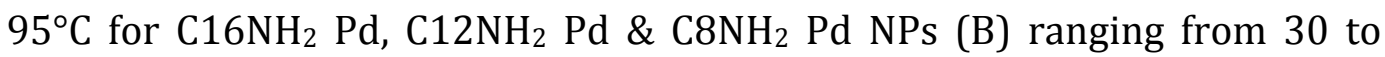
$110^{\circ} \mathrm{C}$ with $\mathrm{C} 16 \mathrm{NH}_{2} \mathrm{Pd} \mathrm{NPs}$ aqueous solution and (C) ranging from $40^{\circ} \mathrm{C}-70^{\circ} \mathrm{C}$ with Pd NPs with all reactants heated for 30 minutes at each temperature. 
acid) as solution drop wise in the reaction mixture to get the products while keeping the catalyst system stabilized.

\subsection{CONCLUSIONS}

We studied the stability of glutathione-coated Pd NPs synthesized under inert and oxygen atmosphere and citrate-coated Pd NPs in solutions in the absence and presence of substrates by bubbling hydrogen gas through the solution and using UV-vis spectroscopy measurements of the NPs as a function of bubbling time. All synthesized glutathione-coated Pd NPs synthesized under inert and oxygen conditions showed high stability under hydrogen atmosphere for several hours. The same trend was observed for all Pd-alloy NPs with Au and Pt with different ratios synthesized under both inert and oxygen atmosphere as expected confirming that the glutathione ligands provide high stability against hydrogen-induced aggregation. The hydrogen stability of Pd-Glu (inert) NPs at different intervals of time under monophasic aqueous solution in the presence of allyl alcohol with $100 \%$ hydrogen also showed high stability. We also studied the stability of Pd-Glu (Inert) NPs and Pd-Glu (Oxy) NPs against hydrogen-induced aggregation under the biphasic condition of chlorobenzene and nanopure water in the presence of allyl alcohol with 100\% hydrogen, which showed higher stability for Pd-Glu (Inert) NPs than Pd-Glu (Oxy) NPs. We also studied the stability of Pd-alloy that is (75:25) Pd:Au-Glu (Inert) NPs under biphasic conditions of nanopure water and chlorobenzene in the absence and presence of allyl alcohol. NPs in the absence of allyl alcohol showed high absorbance with decrease in wavelength showing little formation of $\operatorname{Pd}(\mathrm{II})$ species, 
which increased in the presence of allyl alcohol substrate showing that substrate has some effect on the Pd surface. The Pd-Glu (Inert) NPs showed high stability even after keeping it in aqueous solution for 60 days.

In the case of citrate-coated Pd NPs, we determined the stability of the aqueous Pd-citrate NPs solution without the presence of substrate by monitoring the UV-vis spectra. Citrate-coated Pd NPs showed high stability, although there was some formation of $\mathrm{Pd}(\mathrm{II})$ species that may have leached out from or remained on the palladium core. We studied the stability of Pd-citrate NPs against hydrogen-induced aggregation under the biphasic condition of dichloromethane and nanopure water in the presence of $0.74 \times 10^{-3}$ moles of $\alpha, \beta$-unsaturated alcohols (allyl alcohol, 1penten-3-ol, 1-hepten-3-ol or 2-methyl-3-buten-2-ol). UV-vis spectra for Pd-citrate NPs in the presence of allyl alcohol showed low stability in terms of aggregation and the presence of a high concentration of Pd(II) species formed within the first 5 minutes of the reaction. Pd-citrate NPs in the presence of 1-hepten-3-ol showed a similar trend of low stability against aggregation, but less formation of $\mathrm{Pd}(\mathrm{II})$ species. The presence of 1-penten-3-ol showed comparatively higher stability with a similar spectrum for first 15 minutes and less formation of $\operatorname{Pd}(\mathrm{II})$ species. The presence of 2-methyl-3-buten-2-ol, having the same number of carbon atoms as 1penten-3-ol, also showed high stability and low Pd (II) species formation. Thus, the Pd-citrate NPs were surprisingly stable considering the weakly bounded citrate stabilizer and showed that these NPs are more stable to longer chain allylic alcohols in biphasic solvent mixture. 
In general, Pd NPs stabilized with citrate ligands showed the formation of $\operatorname{Pd}(\mathrm{II})$ species but they were still stable against hydrogen-induced aggregation. These studies provided interesting knowledge of stability of NPs against hydrogen, which is useful for the catalysis studies described in Chapters IV and V.

For determining the thermal stability of various alkylamine-coated Pd NPs for C-C coupling Suzuki-Miyaura reaction, we showed that the absorbance for $\mathrm{C} 16 \mathrm{NH}_{2} \mathrm{Pd} \mathrm{NPs}$ remained high for all temperatures whereas absorbance for $\mathrm{C}_{12} \mathrm{NH}_{2}$ $\mathrm{Pd}$ and $12 \times \mathrm{C} \mathrm{NH}_{2} \quad \mathrm{Pd}$ NPs decreased rapidly at high temperatures showing temperature-induced aggregation and thus, lower stability of these NPs due to weak interaction of amines with the Pd core and shorter chain-length. The absorbance of $\mathrm{C}_{16} \mathrm{NH}_{2}$ Pd NPs remains high for all temperature that shows the overall thermal

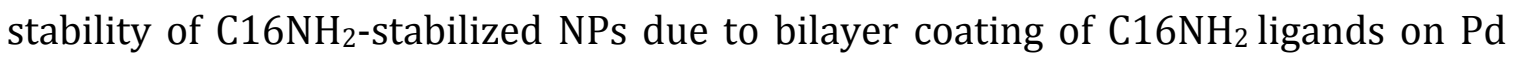
core $^{75}$ and thus thermally suitable for use as a catalyst in the Suzuki reaction. But, when all the components of the Suzuki reaction (toluene, water, $\mathrm{K}_{2} \mathrm{CO}_{3}$, phenylboronic acid, 4-iodoanisole and $\mathrm{C}_{16 \mathrm{NH}_{2}} \mathrm{Pd} \mathrm{NPs}$ ) were stirred at temperatures ranging from $40^{\circ} \mathrm{C}-70^{\circ} \mathrm{C}$ each for 30 minutes, the NPs weren't stable after $60^{\circ} \mathrm{C}$. 


\section{CHAPTER IV}

CONTROLLED ACTIVITY AND SELECTIVITY OF GLUTATHIONE-STABILIZED

Pd, PdPt AND PdAu NANOPARTICLES FOR ISOMERIZATION OF ALLYL ALCOHOL

\subsection{INTRODUCTION}

Here, we studied the reaction of 2-propen-1-ol with hydrogen gas in the presence of reduced-L-glutathione stabilized Pd, PdPt and PdAu NPs to investigate their catalytic properties. Because this allyl alcohol is the simplest $\alpha, \beta$-unsaturated alcohol and has been used as a substrate in previous studies, ${ }^{41,50-52,88,93,94,144-151}$ it was a good starting point for this work. As illustrated in Figure 4.1, the reaction between allyl alcohol and hydrogen gas using Pd catalysts results in hydrogenation of the double bond to give propanol (hydrogenated product) and/or it may lead to migration of double bond in keto-enol tautomerism fashion yielding the corresponding saturated aldehyde. $152-154,159,180$ In the past, various groups have studied the activity and selectivity for hydrogenation and isomerization of allyl alcohols using heterogeneous $\mathrm{Pd}$ catalysts, such as $\mathrm{Pd} / \mathrm{TiO}_{2}, 86,87$ polymer-Pd (0) and Pd (II) complexes, ${ }^{92}$ and Pd NPs stabilized by polymers and immobilized on composites or embedded in polyelectrolyte films. $.^{88}-91$ But all these studies have favored hydrogenation process prohibiting the formation of the isomer. An 


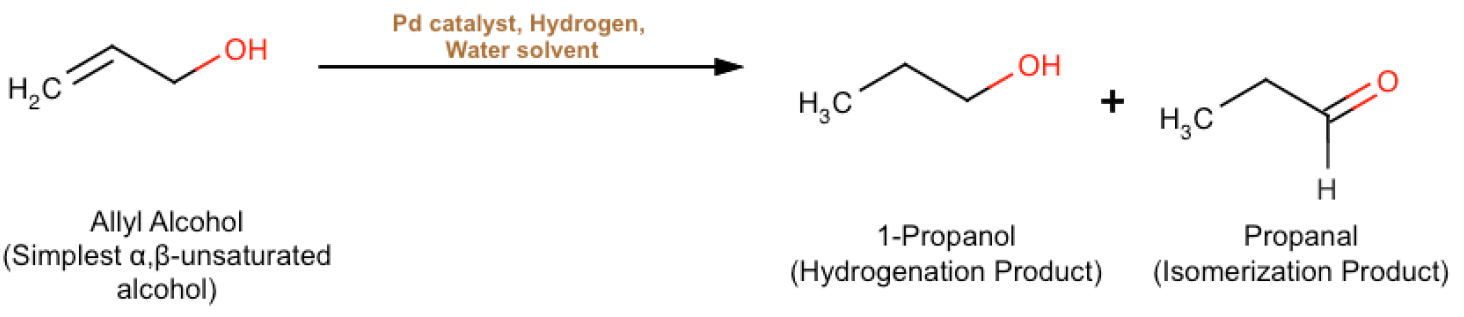

Figure 4.1. The conversion of allyl alcohol to saturated alcohol and aldehyde in the presence of hydrogen and Pd or Pd alloy NP catalyst. 
important challenge is to controllably obtain the saturate isomer or alcohol derivatives, which are essential compounds in organic synthesis. Therefore, our goal was to better understand the catalytic process with different Pd and Pd-alloy NPs to control the selectivity and improve the turnover frequencies and high recyclability by designing stable NPs.

In this study, we describe the catalytic activity of various Pd, PdAu and PdPt NPs synthesized under both aerobic and inert conditions protected with glutathione ligands and their selectivity towards the hydrogenation/isomerization of 2-propen1-ol to form propanol and/or 1-propanal in the presence of hydrogen under biphasic reaction mixture. We hypothesized that the branched, more open structure and water soluble nature of the glutathione ligand would significantly alter the catalytic reactivity and selectivity of the NPs relative to the alkanethiol- and alkylamine-coated Pd NPs synthesized previously in our lab. ${ }^{75}$ Beyond synthetic strategies to optimize the ligand surface of mono-metallic NPs, incorporation of a second metal into the inorganic component and addition of oxygen during synthesis may result in enhancement of the catalytic properties of the system ${ }^{96,181}$ which can be due to geometric arrangement of the metal atoms, altering the interactions with the substrate, or to the electronic character of the overall metallic system based on the different electronegativities of the two metals involved. Here, the more electronegative metal atoms ( $\mathrm{Au}$ and $\mathrm{Pt}$ ) should pull electron density from the second component (Pd), making it more electropositive and more reactive in the catalytic system as this is believed to increase its interactions with the hydrogen and the olefin substrate to enhance the reaction TOF. ${ }^{181}$ Also, Sharma et al. ${ }^{143}$ showed 
that NPs synthesized under oxygen causes expansion in their lattice parameter due to the incorporation of oxygen from $3.88 \AA$ to $4.12 \AA$. We hypothesized that this might result in more open space for hydrogen to adsorb and diffuse into the lattice to form $\mathrm{PdH}_{\mathrm{x}}$, which could result in higher catalytic activity than nanoparticles prepared under inert condition with no lattice parameter expansion. As reported previously, the formation of a surface palladium hydride species $\left(\mathrm{PdH}_{\mathrm{x}}\right)$, either isolated or generated in situ, is a fundamental requirement in the hydrogenation and isomerization of allyl alcohols. $87,152,154,182$ That's why we prepared nanoparticles under both inert and aerobic conditions to compare their catalytic behavior for hydrogenation/isomerization of allyl alcohol. All of the NPs catalyzed the reaction with $100 \%$ yield, but with different reactivity or turnover frequencies (TOFs), depending on the metal composition and the synthetic environment. The reaction showed high selectivity to isomer product (1-propanal) for all nanoparticles studied. NPs synthesized under aerobic conditions showed higher catalytic activity but were less stable than their inert counterparts, which showed lower catalytic activity but higher stability. PdAu alloy NPs showed higher catalytic activity compared to Pd or PdPt NPs due to the higher electronegativity of Au metal, making Pd more electropositive and thus improving $\pi$-coordination of allyl alcohols. Increased flow rates of hydrogen increased the turnover frequency without any measureable aggregation of the nanoparticles. Ethyl acetate as the organic solvent led to higher activity as compared to dichloromethane and chlorobenzene, but lower nanoparticle stability than dichoromethane. Since efficient recovery of the catalyst from the reaction medium after the completion of the reaction is a key 
factor that determines its usage for practical applications, we studied the recyclability of the Pd-glutathione NPs synthesized under inert and air conditions. Inert synthesized NPs showed higher recyclability than air synthesized ones. The glu-protected (75:25) Pd:Au alloy NPs synthesized under inert conditions catalyzed the reaction for nine cycles with a $100 \%$ conversion rate in a dichloromethane/nanopure water biphasic mixture with a turnover frequency (TOF) 400 moles product/moles $\mathrm{Pd} /$ hour. The most reactive catalyst was the glutathione-protected (50:50) Pd:Au alloy NPs synthesized in air with $20 \mathrm{~mL} / \mathrm{min}$ hydrogen flow rate, which displayed a TOF of 772 moles product/moles Pd/hour, but with low recyclability. The reaction products were easily removed from the organic phase and the catalyst could be reused several times with the same aqueous phase without needing to precipitating and re-dissolve the NPs. The catalytic performances of these glutathione Pd and Pd alloy NPs compare favorably to other types of alkanthiol, alkylamine, and other stabilized Pd NPs reported in the literature. The branched, open structure and their use in a biphasic solvent provide high catalytic activity and recyclability, while the thiol group makes it highly stable and selective to the isomer.

\subsection{RESULTS AND DISCUSSION}

In order to design a catalyst with high stability, reactivity and recyclability and to study the role of the presence of oxygen and role of ligands as gates/filters in selectively catalyzing various organic reactions, Pd and Pd alloy NPs co-reduced with Au or Pt in different metal compositions (Pd:Au/Pt in 90:10, 75:25 and 50:50) 
stabilized with glutathione ligands under both inert and oxygen atmosphere were synthesized as described in Chapter II and we compared their catalytic behavior for the hydrogenation/isomerization reaction of allyl alcohol. We used 3 different flow rates of hydrogen $(8.0 \pm 0.2,19.9 \pm 0.7$ and $39.9 \pm 0.3 \mathrm{~mL} / \mathrm{min})$ during the catalytic reactions to observe the change in turnover frequencies (TOF) and selectivity of various synthesized NPs. We also used different organic solvents, including chlorobenzene, dichloromethane and ethyl acetate with nanopure water as biphasic solvent system for easy separation of catalyst and products. These organic solvents were choosen because they don't interfere with the GC of substrates and products used. They also affect the catalytic behavior of the NPs used during the reaction. We observed a little formation of isomerized product $(\sim 6 \%)$ even in the absence of hydrogen, which could be due to the presence of NPs allowing keto-enol tautomerism to some extent. We didn't observe any reaction without catalyst in the presence of hydrogen and all of the substrate was recovered quantitatively. We monitored the homogeneous biphasic liquid-liquid catalysis and selectivity for the hydrogenation/isomerization of allyl alcohol with various glutathione-coated Pdand Pd-alloy NPs and compared them to $\mathrm{C} 6 \mathrm{~S}, \mathrm{C} \mathrm{NH}_{2}, \mathrm{C} 12 \mathrm{NH}_{2}$ and $\mathrm{C} 16 \mathrm{NH}_{2}$-coated Pd NPs synthesized previously in our lab. ${ }^{75}$ The glutathione coated NPs are well soluble in water and thus, could be utilized as a homogeneous catalyst for green chemistry. Also, all these glutathione-coated Pd and Pd-alloy NPs are air and moisture-insensitive and thus, can be kept for a long time (at least 2 years) without any measurable change in their catalytic activity. These NPs can be easily separated from the products under biphasic conditions that we utilize in all catalytic reactions 
and thus, there is no product lost. Products was dissolved in the organic solvent which can be removed by simple extraction whereas the catalyst remains dissolved in the aqueous phase and can be reused several times. Also, adding 2 times volume of THF solvent to the aqueous phase aliquot can easily crash these NPs for convenient further analysis. Since the Pd core of these NPs is attached to glutathione through strong Pd-S interactions, they are very stable even under high flow rates of hydrogen $(39.9 \pm 0.3 \mathrm{~mL} / \mathrm{min})$.

\subsubsection{Biphasic catalysis with glutathione-coated NPs}

For the hydrogenation/isomerization reaction of allyl alcohol to propanol and 1-propanal, we dissolved glutathione-coated Pd and Pd-alloy NPs in nanopure water and added organic solvent (chlorobenzene, dichloromethane or ethyl acetate) as shown in Figure 4.2. Allyl Alcohol was added and hydrogen gas purged at a particular flow rate $(8.0 \pm 0.2,19.9 \pm 0.7$ and $39.9 \pm 0.3 \mathrm{~mL} / \mathrm{min})$. Aliquots of the solution were taken after particular intervals of time and the NPs precipitated for GC analysis by adding THF solvent and centrifuging it at $4000 \mathrm{rpm}$ for about 15 minutes. Figure 4.3. shows several gas chromatograms (GC) of the aqueous and organic phase at different times of $0,5,10,15,20$ and 25 min during the reaction of allyl alcohol with hydrogen $(19.9 \pm 0.7 \mathrm{~mL} / \mathrm{min})$ catalyzed by Pd-Glu (Oxy) NPs in a biphasic solvent mixture of $2 \mathrm{~mL}$ nanopure water and $2 \mathrm{~mL}$ chlorobenzene. In the aqueous phase, the peak corresponding to allyl alcohol at a retention time of 4.4 minutes decreased with time, whereas the peaks corresponding to propanal and 1propanol at 1.5 and 3.6 minutes, respectively, increased with time. In the organic 


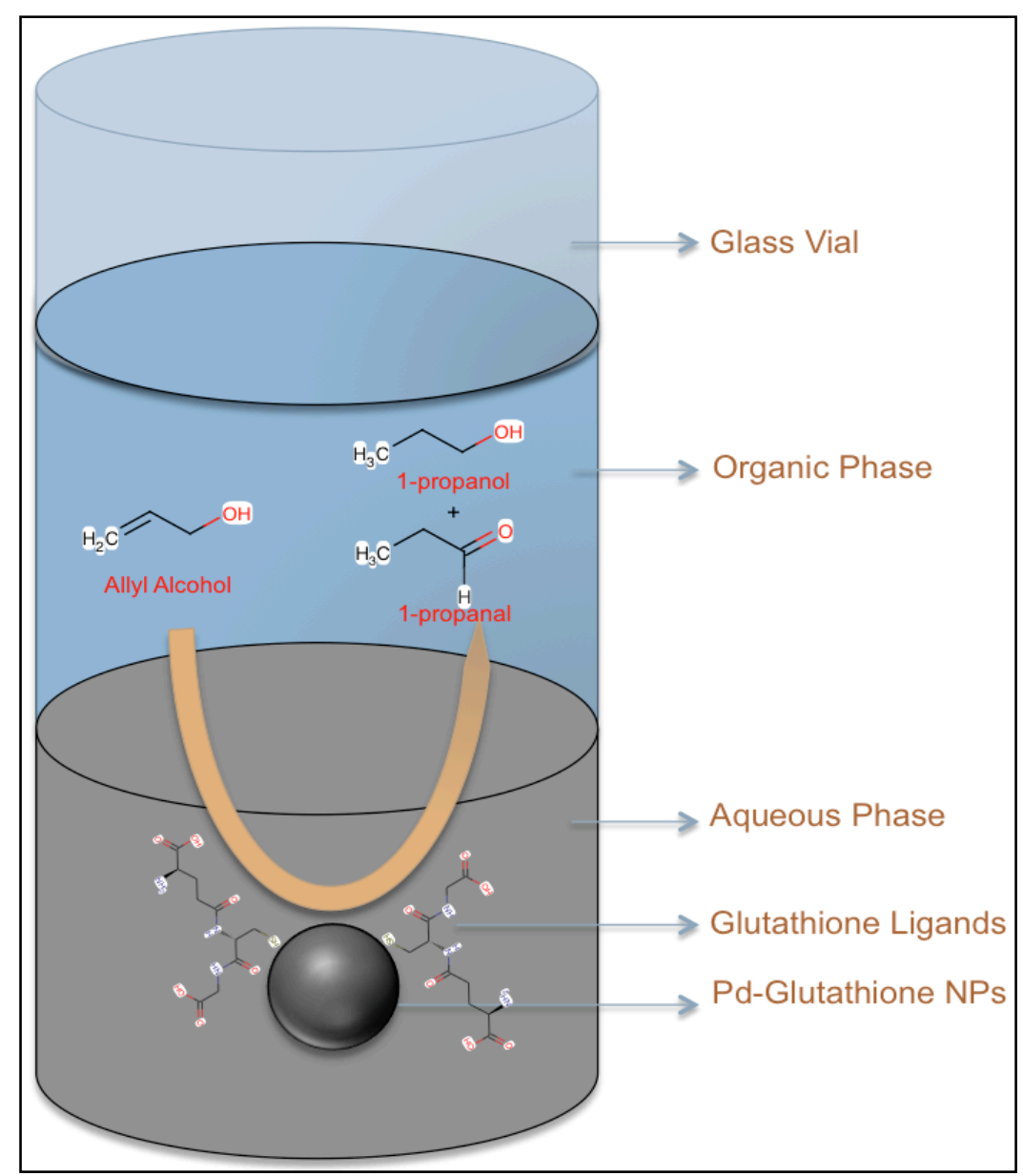

Figure 4.2. Schematic illustration of a biphasic catalytic reaction using $2 \mathrm{~mL}$ of organic solvent (ethyl acetate, dichloromethane or chlorobenzene) and 2 $\mathrm{mL}$ of nanopure water and glutathione-coated Pd NPs as a catalyst for the conversion of allyl alcohol as a substrate. 


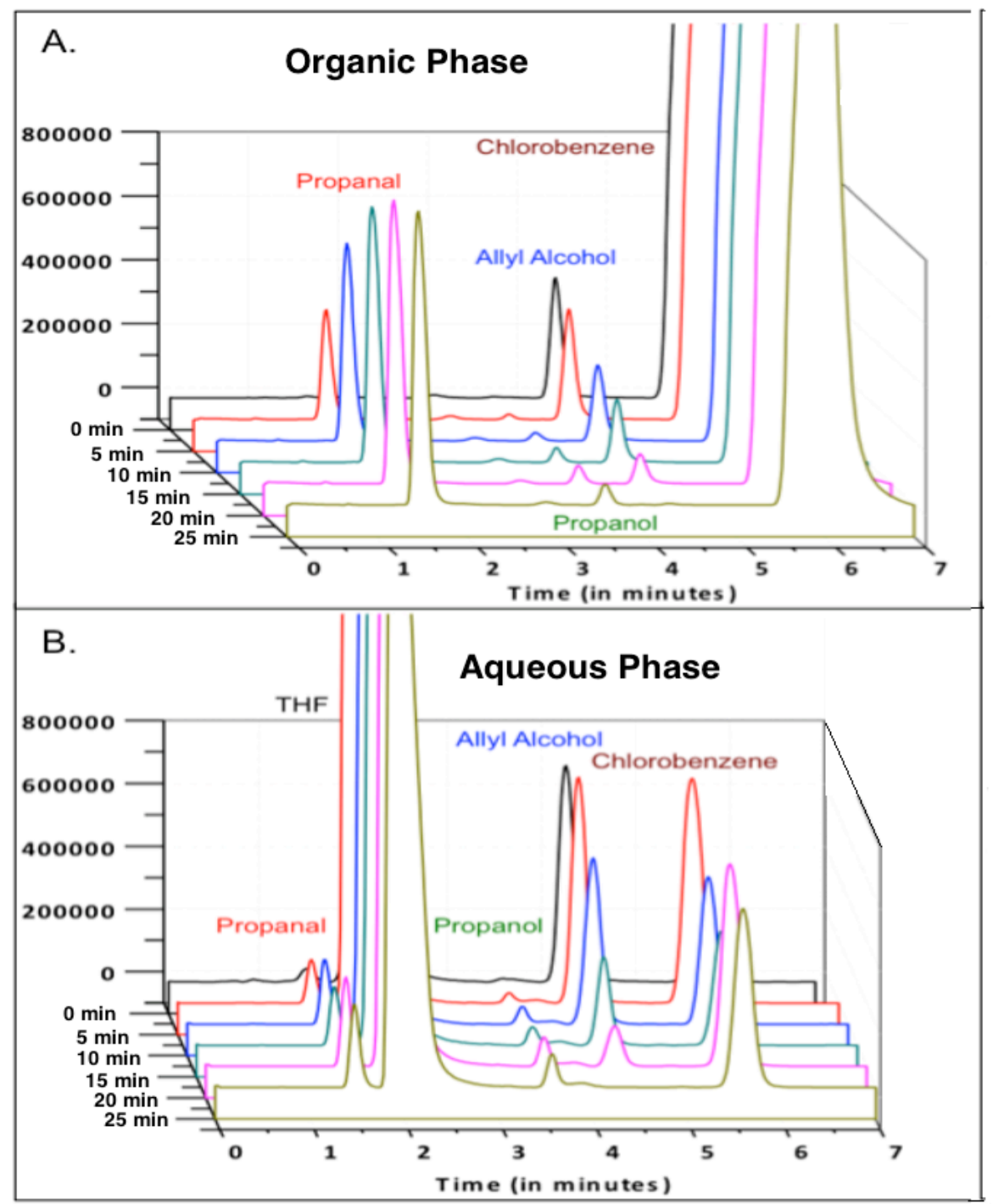

Figure 4.3. Gas chromatograms of organic layer (A) and aqueous layer (B) of a biphasic solvent mixture showing the progress of the catalytic reaction of allyl alcohol before and after exposure to $100 \%$ hydrogen $(19.9 \pm 0.7 \mathrm{~mL} / \mathrm{min})$ at different reaction times of $0,5,10,15,20$ and 25 minutes. The reaction was catalyzed by $\sim 6.0 \mathrm{mg}$ of Pd-glu (Oxy) NPs in biphasic solvent mixture of $2 \mathrm{~mL}$ water and $2 \mathrm{~mL}$ chlorobenzene under atmospheric pressure and at room temperature. Integration of peaks for analysis was performed using instrument software. Injection volume $=1 \mu \mathrm{L}$, initial temperature $=80^{\circ} \mathrm{C}$, final temperature $=135^{\circ} \mathrm{C}, \operatorname{ramp}=25^{\circ} \mathrm{C} / \mathrm{min}$, pressure $=12-14$ PSI, Detector $=$ FID. 
phase, the peak heights for both products increased more significantly with time as the allyl alcohol peak decreased and eventually vanished, showing the presence of most of the product in the organic phase. This makes it easy to separate product from the aqueous phase and the catalyst with no product loss. The catalyst remained dissolved in the aqueous phase, which was recovered and reused several times. Pd-Glu (Oxy) NPs are clearly active catalysts and selective for the isomerization reaction as indicated by complete loss of allyl alcohol and the much bigger peak for the propanal product in the GC. We observed very small changes in the UV-Vis spectrum of the Pd-Glu NPs, suggesting some small irreversible reactivity with hydrogen, but the GC data confirms that these NPs are very active catalysts. Shon and co-workers recently described the same selectivity for the isomer with alkanethiolate-capped Pd NPs prepared from Bunte salts. ${ }^{81}$ 80, 132, 133 Figure 4.4. shows the percent hydrogenation, percent isomerization, and total conversion of allyl alcohol at different times when Pd-glu (Oxy) NPs were used as catalysts.

Our motivation behind synthesizing Pd-containing alloy NPs was based not only on atom economy but also to enhance the selectivity and catalytic activity of the synthesized NPs. Figure 4.5. shows a plot of TOF for all synthesized Pd and Pd-alloy NPs at $19.9 \pm 0.7 \mathrm{~mL} / \mathrm{min}$ hydrogen flow rate using chlorobenzene organic solvent along with the aqueous phase. It clearly shows that the presence of other electronegative metal in the NP increases the overall turnover frequency (TOF) of the reaction, whereas mono-metal nanoparticles show lower catalytic activity. The catalytic activity of the Pd-alloys increased as the ratio of the other metal to Pd increases, with a maximum of 50:50 for Pt:Pd and Au:Pd alloy NPs. The NPs 


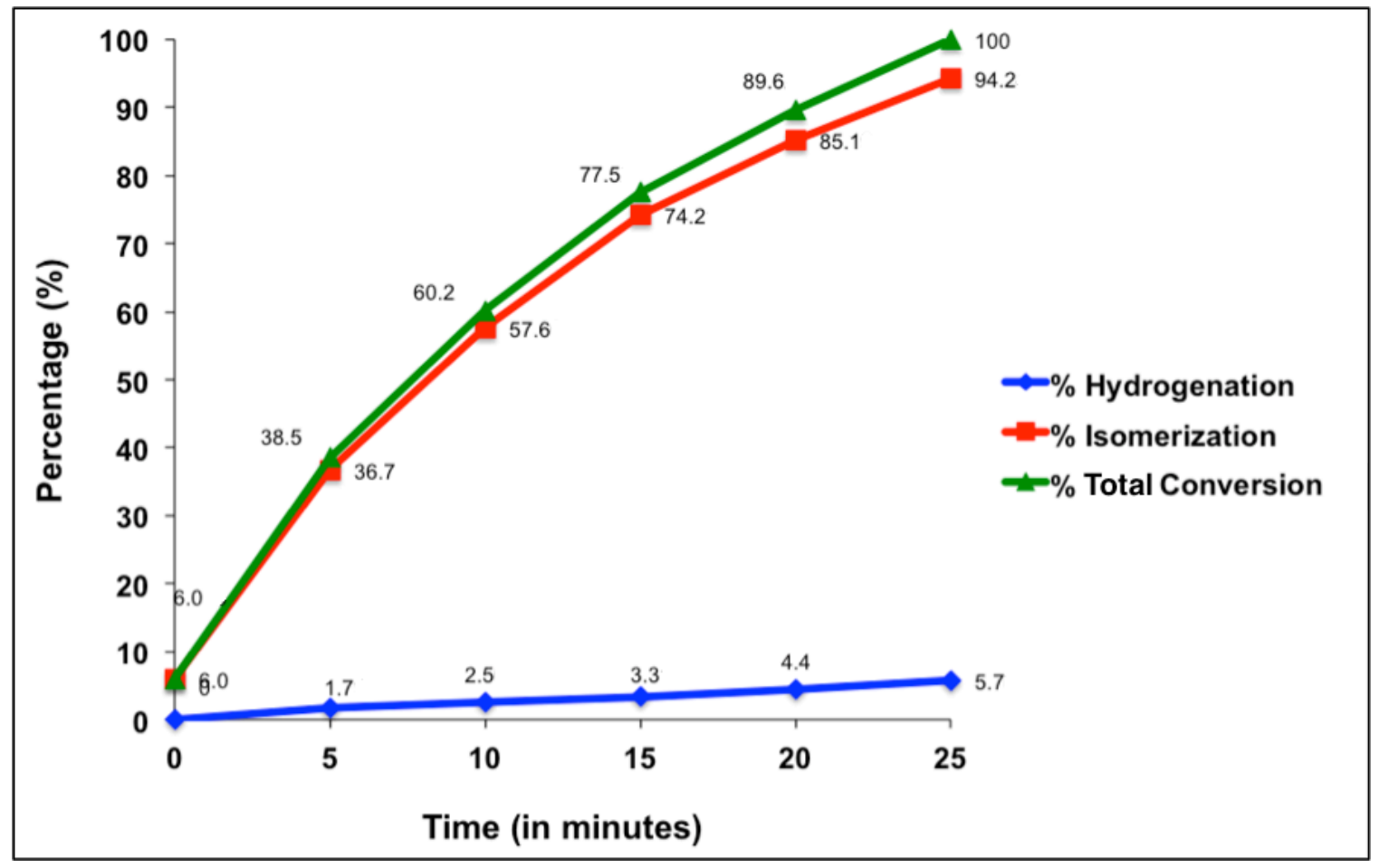

Figure 4.4. Plot of the \%hydrogenation, \%isomerization and \%total conversion of allyl alcohol versus reaction time using Pd-Glu (Oxy) NP catalysts in biphasic solvent mixture of chlorobenzene and nanopure water. 


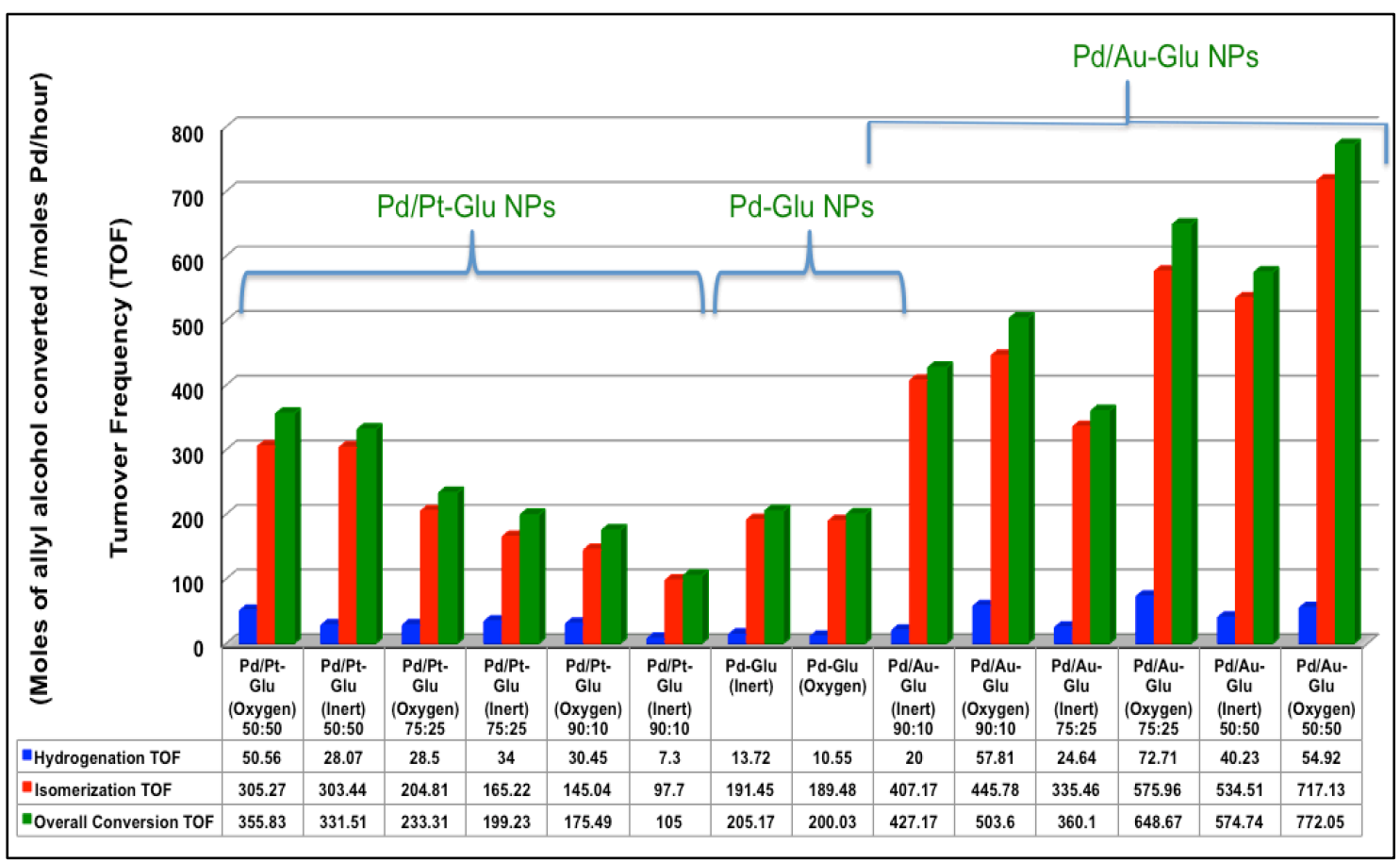

Figure 4.5. Plot of turnover frequencies (TOF, in moles product/moles $\mathrm{Pd} /$ hour) towards hydrogenated and isomerized products for all synthesized $\mathrm{Pd}$ and Pd-alloy NPs under inert/oxygen conditions at $19.9 \pm 0.7 \mathrm{~mL} / \mathrm{min}$ hydrogen flow rate using chlorobenzene organic solvent with aqueous phase. 
synthesized under oxygen atmosphere showed higher catalytic-activity that NPs synthesized under inert conditions, particularly for the Pd alloy NPs. The Pd:Au-Glu (Inert/Oxygen) NPs in all ratios has better TOFs than Pd:Pt-Glu (Inert/Oxygen) NPs with same ratios which shows that the presence of a metal with higher electronegativity like Au with 2.54 than Pt with 2.28 has a significant role in improving their overall catalytic activity of Pd with 2.20 electronegativity. It may also improve the geometry of the crystal lattice, allowing more active sites to be available for catalysis. ${ }^{96,181}$

All NPs showed higher selectivity towards the isomerized product (> 80\%). The highest turn over frequency (TOF) for the catalytic reaction was shown by (50:50) Pd:Au-Glu (Oxy) NPs with 772 moles of product converted/moles Pd/hour. Alkanethiol coated Pd NPs synthesized in our lab previously showed a maximum of 47 TOF for C6S Pd NPs with a selectivity of $>95 \%$ isomer product. The TOF for $\mathrm{C}_{8 \mathrm{NH}_{2}}$ Pd NPs was 120,194 for $\mathrm{C}_{12 \mathrm{NH}_{2}}$ Pd NPs and 719 for $\mathrm{C}_{16 \mathrm{NH}_{2}}$ Pd NPs with the selectivity of $1: 1$ or $3: 2$ ratio towards the hydrogenation:isomerization product. Also, the stability of the alkylamine-coated NPs was poor due to weak interaction of the ligands with the Pd core. With glutathione and the biphasic system, we have successfully prepared a catalyst that is stable, highly active, and easily separated from products.

\subsubsection{Selectivity of glutathione-coated Pd and Pd-alloy NPs}

Figure 4.6. shows that all of the Pd-Glu NPs are selective towards the isomer product. The mono Pd NPs show a slightly higher selectivity towards the isomerized 


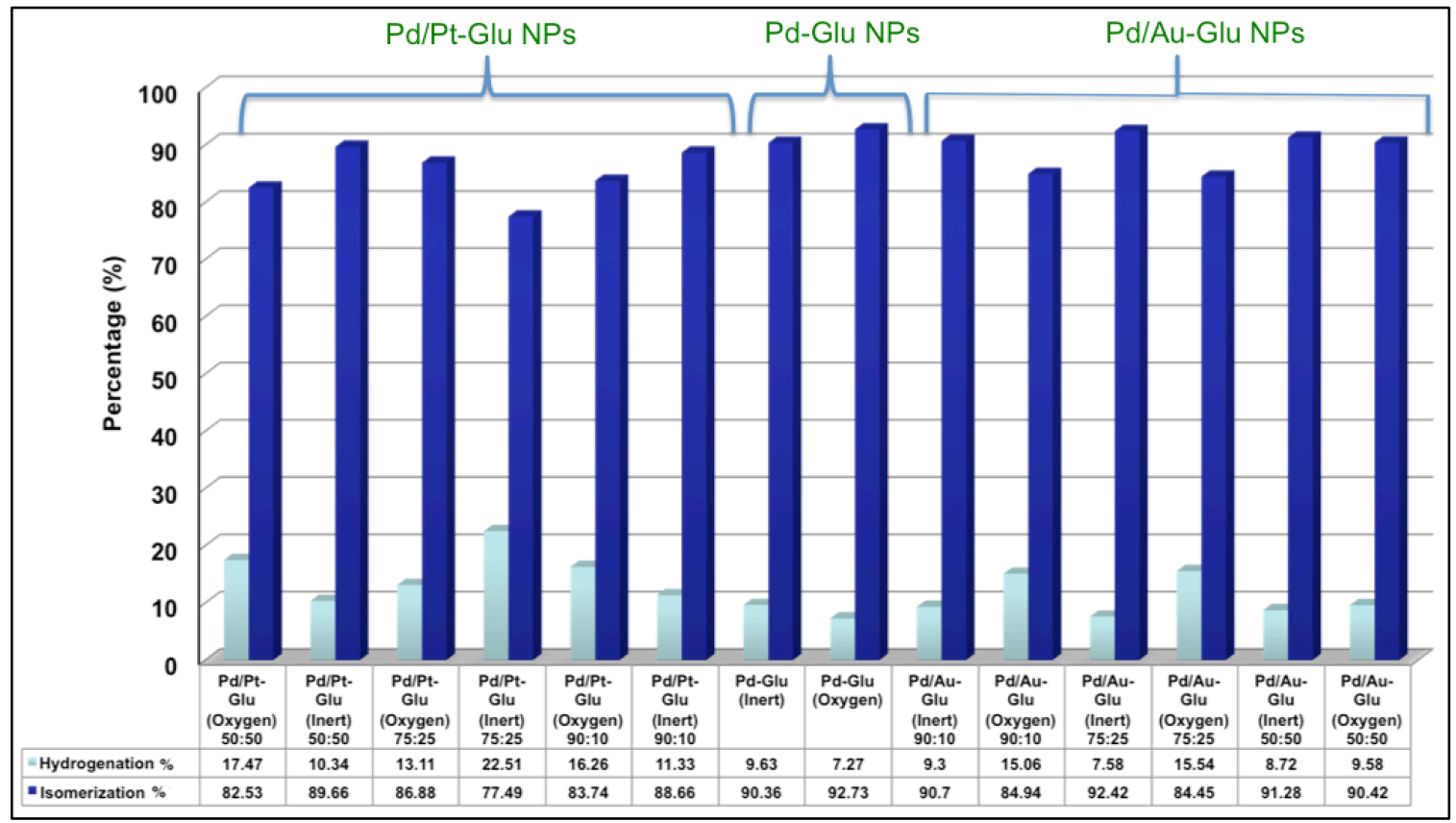

Figure 4.6. Box plot showing the reactivity towards the hydrogenated and isomerized products after one complete cycle of reaction between allyl alcohol and 100\% hydrogen at $19.9 \pm 0.7 \mathrm{~mL} / \mathrm{min}$ flow-rate using chlorobenzene and water biphasic solvent system for all synthesized glutathione-coated NPs [Pd-, Pd/Pt- and Pd/Au-glu (Inert/Oxygen) NPs]. All NPs are highly selective towards the isomer product. 
product as compared to alloy NPs, although all NPs showed selectivity of $<75 \%$ for the isomerized product. These results suggested that the selectivity depends mainly on the ligand composition and little on the metal composition of the NPs under the same reaction condition.

The isomerization reaction is an important one-pot catalytic transformation to carbonyl compounds that proceeds with high efficiency, selectivity and atom economy, avoiding the two-step sequential oxidation and reduction reactions. Sensitive substrates do not survive the conditions involving the use of toxic and/or expensive oxidation and reduction reagents and therefore, catalytic redox isomerization is especially useful under mild reaction conditions as shown here. The formation of a higher concentration of isomerized product is one of the main advantages. Also, the strategy is attractive as little to none byproducts are formed. Only saturated aldehydes or ketones are formed which are valuable intermediates for various pharmaceuticals, agrochemicals and fine chemicals.154 These isomerization reactions can be used in multi-step synthesis of high value added compounds in organic chemistry like naturally occurring pheromones like muscone ${ }^{155}$ and (+)-iso-exo-brevicomin, ${ }^{156}$ the marine alkaloid (-)-brevisamide, ${ }^{153}$ the fragrance Florhydral ${ }^{157}$ and the antitumor agent (-)-FR182877,158 all of which includes a redox isomerization step. For all these syntheses, Pd NPs stabilized with glutathione ligands has shown great importance due to their high stability and high reactivity. 


\subsubsection{Role of different flow rates of hydrogen on turnover frequency (TOF) and selectivity of the catalytic reaction.}

It is important to study the effect of flow rate of hydrogen on the TOF and the selectivity of the overall reaction, as hydrogen is one of the reactants in the hydrogenation/isomerization of allyl alcohol. We have used 3 different flow rates of $100 \%$ hydrogen $(8.0 \pm 0.2,19.9 \pm 0.7$ and $39.9 \pm 0.3 \mathrm{~mL} / \mathrm{min})$ using chlorobenzene solvent for observing change in TOF and selectivity for Pd-Glu (Inert/Oxygen) NPs. It was observed that the TOF increased with an increase in the flow rate of hydrogen passing through the reaction mixture. The TOFs almost doubled as we increased the hydrogen flow rate from $8.0 \pm 0.2$ to $39.9 \pm 0.3 \mathrm{~mL} / \mathrm{min}$ as shown in Figure 4.7. (A). We also studied the selectivity of the reaction for the hydrogenated/isomerized product using Pd-Glu (Inert/Oxy) nanoparticles at all three 8.0 $\pm 0.2,19.9 \pm 0.7$ and $39.9 \pm 0.3 \mathrm{~mL} / \mathrm{min}$ flow rates of $100 \%$ hydrogen using chlorobenzene solvent as shown in Figure 4.7. (B). The selectivity for all the reactions showed more than $90 \%$ preference for the isomerized product in all cases, which showed that increasing the flow rates of hydrogen had no general effect on the selectivity. It appears to depend only on the attached ligands. We believe that Pd-S interaction is the key in the selectivity, since several different types of thiolates give the same isomer selectivity.

We also studied the total percent completion of the reaction with time (minutes) for Pd-Glu (Inert) NPs at $8.0 \pm 0.2,19.9 \pm 0.7$ and $39.9 \pm 0.3 \mathrm{~mL} / \mathrm{min}$ hydrogen flow rates using chlorobenzene and aqueous phase which clearly showed that at lowest flow rates, it took at least the first 5 minutes for NPs to become catalytically active as compared to the higher flow rates, which showed activity from 

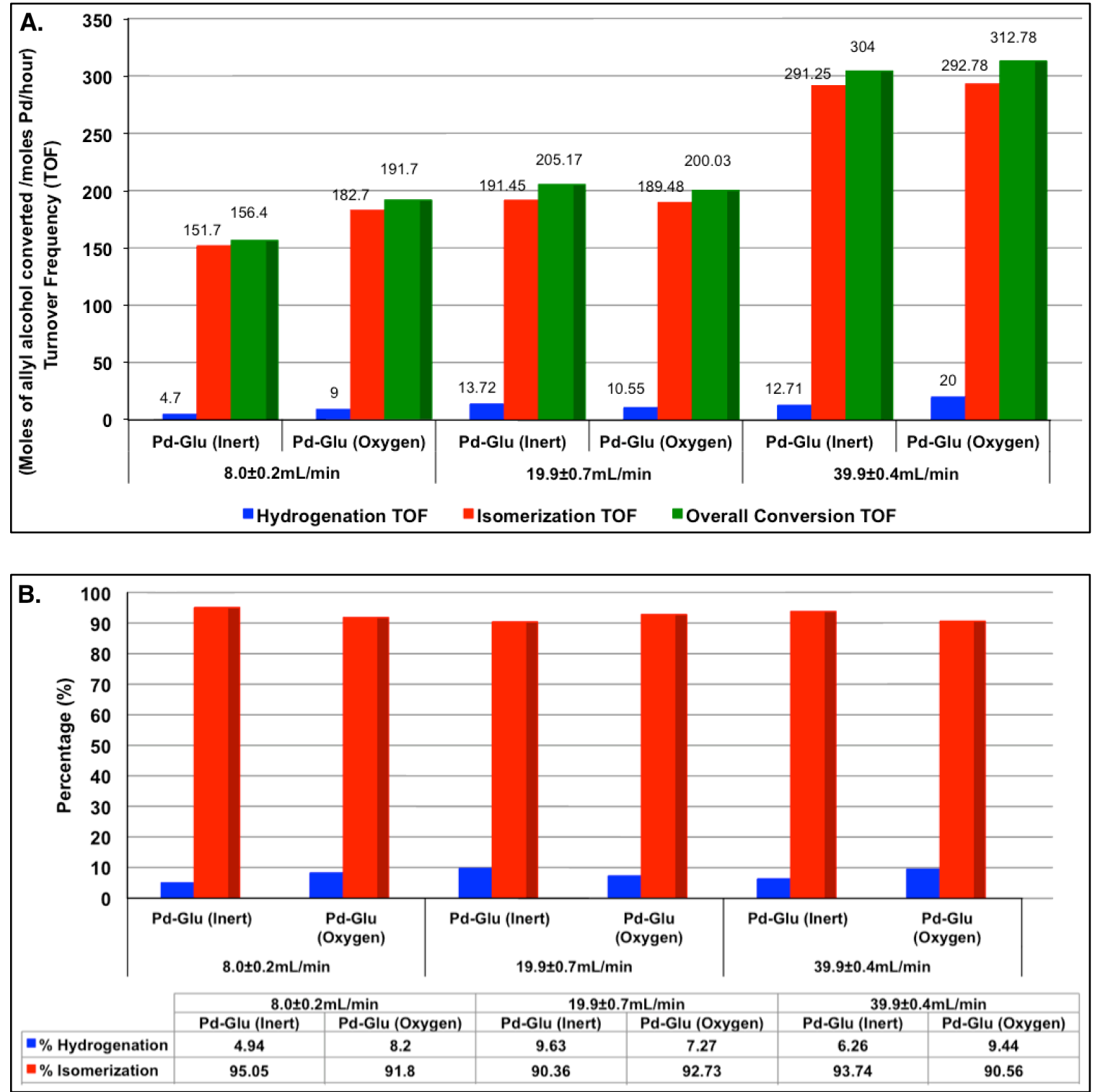

Figure 4.7. Effects of three different flow rates of $100 \%$ hydrogen $(8.0 \pm 0.2$, $19.9 \pm 0.7$ and $39.9 \pm 0.3 \mathrm{~mL} / \mathrm{min}$ ) on (A) turnover frequencies (TOF) and (B) selectivity of the overall catalytic reaction using Pd-Glu (Inert/Oxygen) NPs. 


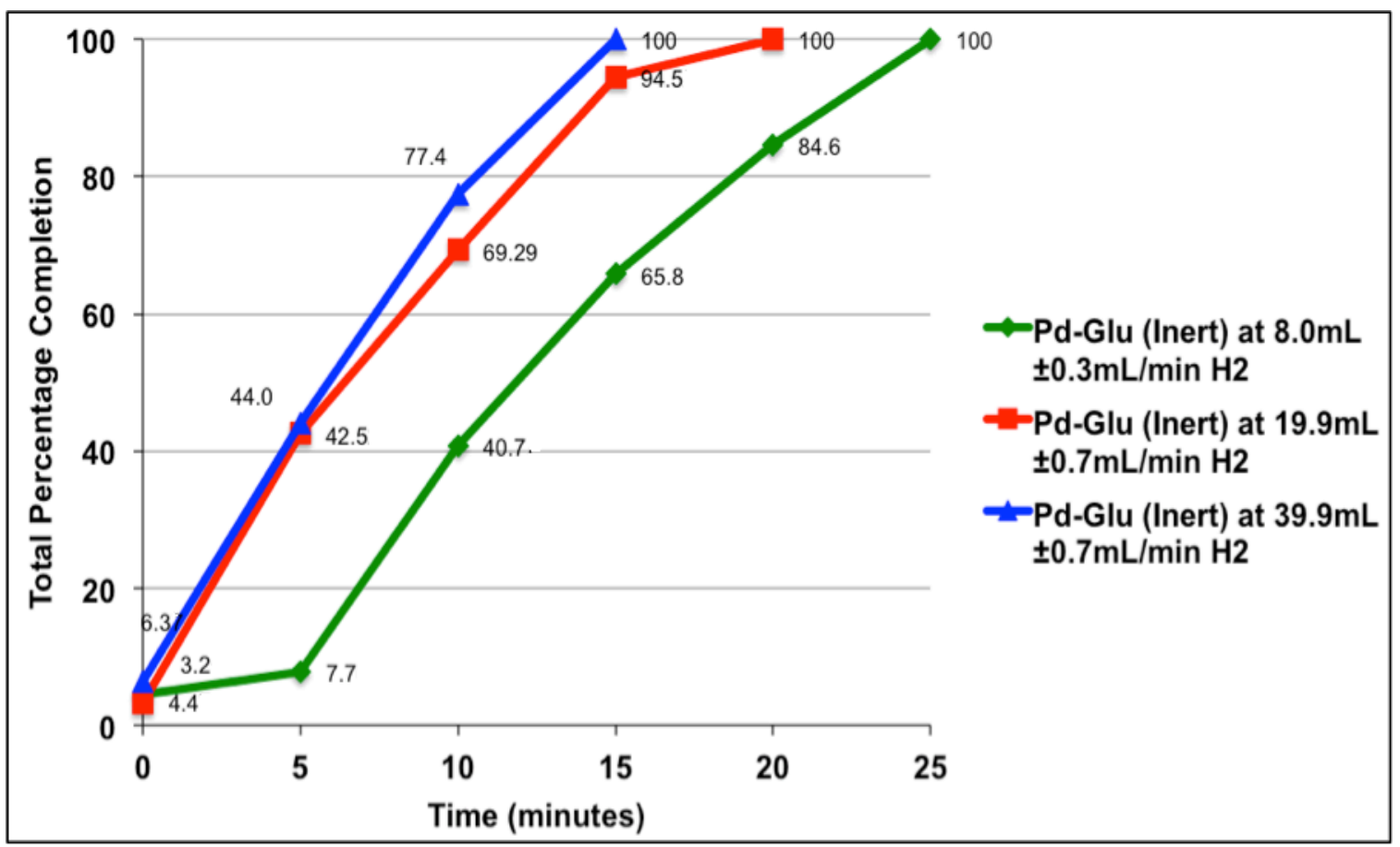

Figure 4.8. Plot showing the total percent completion of the catalytic reaction vs. time (minutes) for Pd-Glu (Inert) NPs at $8.0 \pm 0.3,19.9 \pm 0.7$ and $39.9 \pm 0.7 \mathrm{~mL} / \mathrm{min}$ hydrogen flow rate using chlorobenzene and aqueous phase. 
the beginning of the reaction as shown in Figure 4.8. The reason for this is not clear at this time.

\subsubsection{Role of different organic solvents in the biphasic mixture on turnover frequency (TOF) and selectivity of the catalytic reaction.}

Since different solvation of NPs provide different protecting environment, studying different combinations of biphasic solvent system with aromatic, nonaromatic or non-halogenated solvent is very important for stable NPs with high reactivity. Also, different solvents have different solubility for hydrogen gas, which is important for providing access of hydrogen to the reactant on the Pd surface. Thus, we used (75:25) Pd:Au-Glu (Inert/Oxy) NPs as our model catalysts at 19.9 \pm 0.7 $\mathrm{mL} / \mathrm{min}$ hydrogen flow rate for comparing their catalytic activity (TOF) in three organic solvents ethyl acetate (EtOAc), chlorobenzene (Clben) and dichloromethane (DCM) as shown in Figure 4.9. (A).

TOF values obtained shows that ethyl acetate solvent is better for catalysis with highest TOFs than both chlorobenzene and dichloromethane, although the selectivity plot in Figure 4.9. (B) shows that ethyl acetate solvent a higher hydrogenated product (1-propanol) than chlorobenzene and dichloromethane. The biphasic catalysis with chlorobenzene shows higher TOFs, when Pd-Glu (Oxy) NPs are used but lower TOFs when Pd-Glu (Inert) NPs are used, as compare to using dichloromethane as the organic phase. In general, Pd-alloy NPs synthesized under oxygen shows higher TOFs than their inert counterparts in the presence of all three organic solvents. 

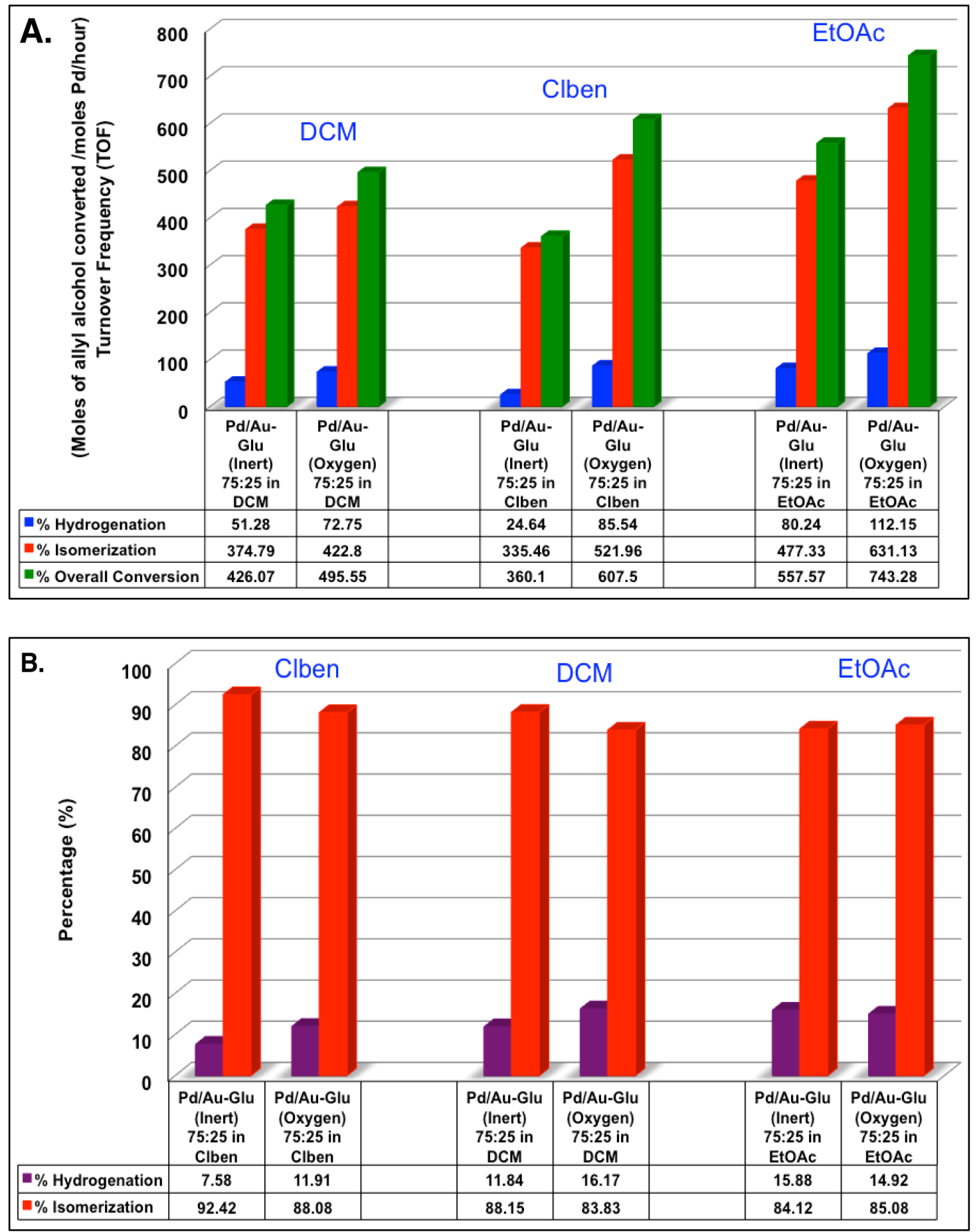

Figure 4.9. Effects of three different organic solvents (DCM, EtOAc, Clben) on (A) turnover frequencies (TOF) and (B) selectivity of the overall catalytic reaction using (75:25) Pd:Au-Glu (Inert/Oxygen) NPs. 


\subsubsection{Stability comparison of Pd-Glu (Inert) NPs with previously synthesized C6S-Pd NPs.}

To compare the stability of Pd-Glu (Inert) NPs with C6S-Pd NPs synthesized previously in our lab, ${ }^{75}$ we studied the hydrogenation and isomerization turnover rates of allyl alcohol by Pd-Glu (Inert) NPs at all three different flow rates of $100 \%$ hydrogen $8.0 \pm 0.2,19.9 \pm 0.7$ and $39.9 \pm 0.3 \mathrm{~mL} / \mathrm{min}$ as shown in Figure 4.10. (A). These plots show that although both Pd-Glu (Inert) NPs and C6S-Pd NPs are stabilized by thiol-coated ligands, Pd NPs coated with glutathione have greater stability, especially at $39.9 \pm 0.3 \mathrm{~mL} / \mathrm{min}$ as compared to C6S-Pd NPs, which become unstable and start precipitating at higher hydrogen flow rates [Figure 4.10. (B)]. This reveals that glutathione-coated Pd NPs are much stable and a more suitable candidate for catalytic applications.

\subsubsection{Recyclability of glutathione-coated Pd and Pd-alloy NPs.}

Since maximizing the recycling capacity of the catalysts decreases the overall cost of the production of chemical products significantly at the industrial scale, it's very important to study the recyclability of these synthesized Pd and Pd-alloy NPs. Using the biphasic solvent system with nanopure water and organic solvent that are immiscible to each other, it makes it very easy to separate the catalyst from the products that goes into the organic phase and can be easily removed by simple extraction and separation from aqueous solution containing the catalyst. The catalyst dissolved in the aqueous phase can be easily reused for another cycle of hydrogenation/ isomerization reaction of allyl alcohol. 

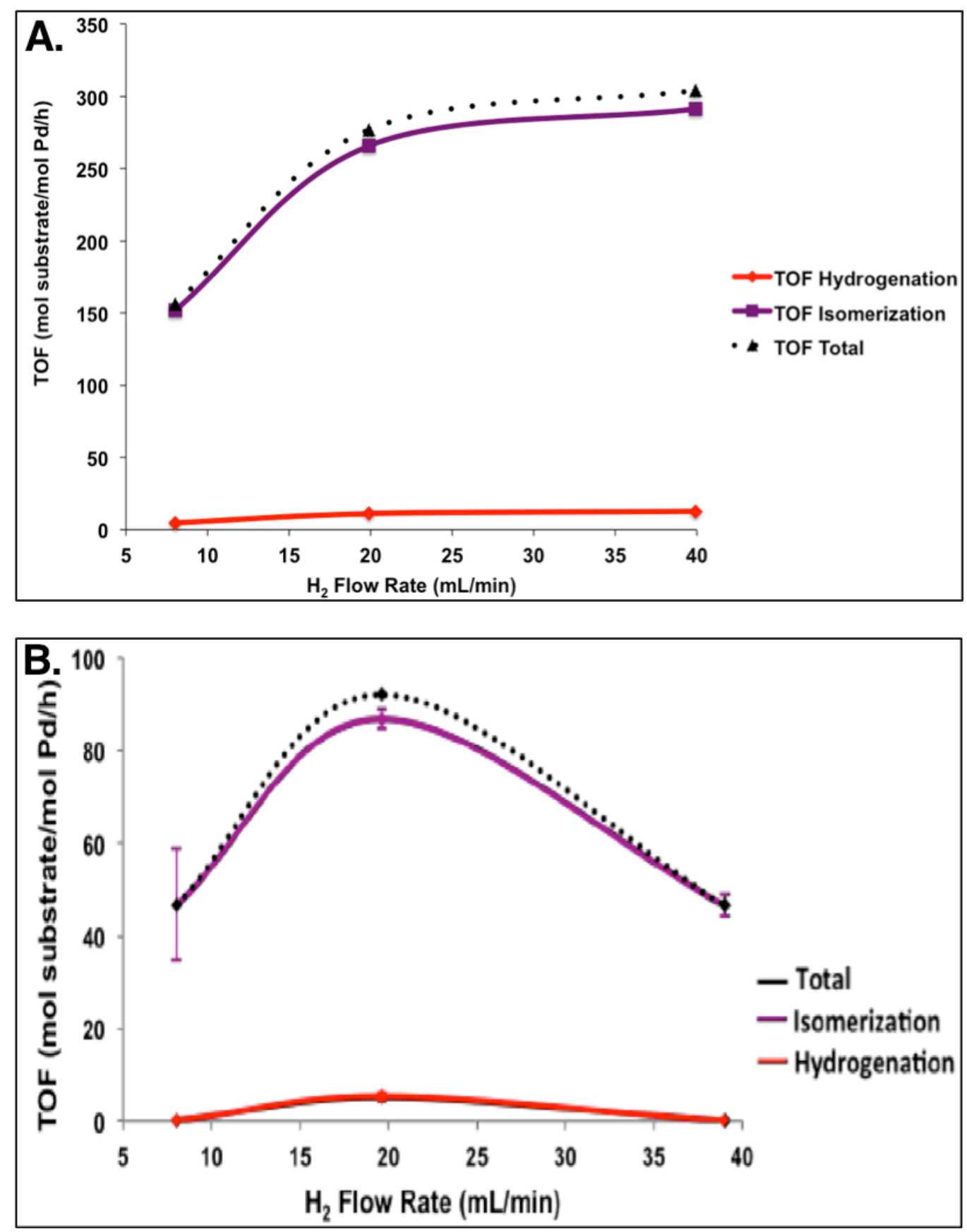

Figure 4.10. Plots showing comparison of hydrogenated-, isomerized- and overall-TOFs for (A) Pd-Glu (Inert) NPs and (B) C6S-Pd NPs ${ }^{75}$ at 3 different flow rates of $100 \%$ hydrogen $(8.0 \pm 0.3,19.9 \pm 0.7$ and $39.9 \pm 0.3 \mathrm{~mL} / \mathrm{min})$. 
The recyclability of (75:25) Pd:Au-Glu (Inert) NPs showed 100\% completion of the catalytic reaction for at least 9 times with dichloromethane at $8.0 \pm 0.2 \mathrm{~mL} / \mathrm{min}$ hydrogen flow rate as shown in Figure 4.11. Each catalytic reaction involves the addition of $6.0 \mathrm{mg}$ of (75:25) Pd:Au-Glu (Inert) NPs into $2 \mathrm{~mL}$ of nanopure water and adding $2 \mathrm{~mL}$ of dichloromethane solvent. $200 \mu \mathrm{L}$ of allyl alcohol was then injected into the reaction mixture. Hydrogen gas was purged through the reaction mixture at a flow-rate of $8.0 \pm 0.3 \mathrm{~mL} / \mathrm{min}$ and $20 \mu \mathrm{L}$ of samples were taken after 45 minutes from both aqueous layer and organic layer separately for GC-FID. The dichloromethane layer with products was separated out from the aqueous layer containing the catalyst. $2 \mathrm{~mL}$ more of dichloromethane was added and removed to extract the remaining product from the aqueous layer. Then, a fresh $2 \mathrm{~mL}$ of dichloromethane solvent was added and a fresh $200 \mu \mathrm{L}$ of allyl alcohol was injected into the reaction mixture. A new reaction was started in the presence of hydrogen gas purging through the solution. (75:25) Pd:Au-Glu (Inert) NPs in aqueous sample solution were crashed by adding $40 \mu \mathrm{L}$ (2xtimes) of THF and centrifuging it for 15 minutes. This procedure was repeated for 12 times and the reaction was monitored by GC-FID. At least 9 reaction cycles were completed with $100 \%$ conversion within 45 minutes. Here, the aqueous phase shows the peaks corresponding to left over products (propanal and 1-propanol) after organic wash and THF solvent and allyl alcohol, which doesn't show up until the $9^{\text {th }}$ cycle showing the complete conversion of allyl alcohol to product. The same pattern was observed in the organic phase. The GC-FID for refill organic layer shows the peaks for allyl alcohol after its addition to 


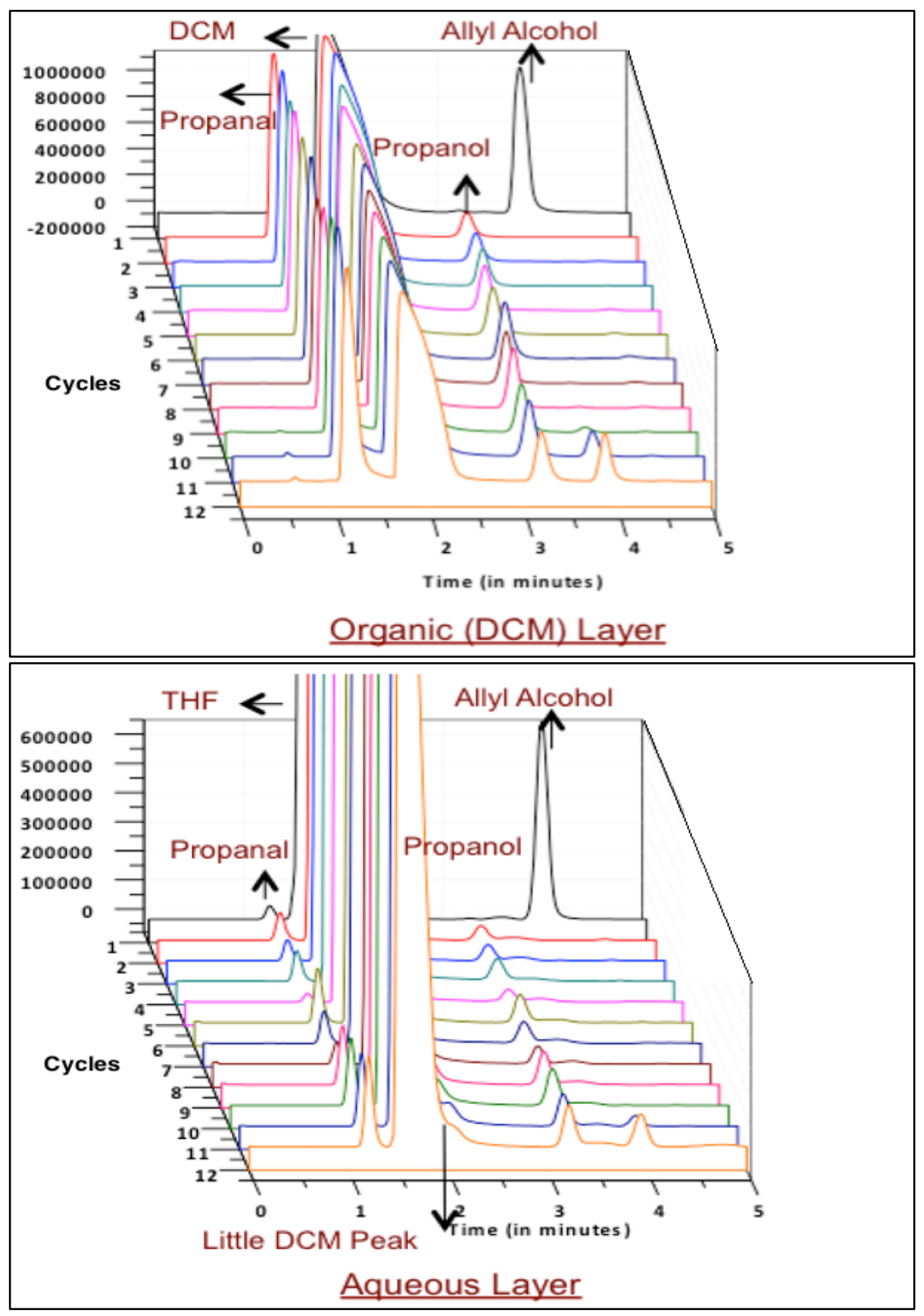

Figure 4.11. GC-FID chromatograms of aqueous and organic phases for the recyclability of (75:25) Pd:Au-Glu (Inert) NPs showing 100\% completion of the catalytic reaction for at least 9 times (checked 12 times) with dichloromethane at $8.0 \pm 0.2 \mathrm{~mL} / \mathrm{min}$ hydrogen flow rate. 
the fresh cycle along with little peaks of the products that came from the previous cycle. THF solvent was used to precipitate Pd NPs since THF peak didn't interfere with the other peaks of reactant and products in GC-FID. Shon and co-workers reported that Pd NPs generated from S-dodecylthiosulfate showed $\sim 75 \%$ isomerization conversion after ten additional repeated cycles. ${ }^{80}$ Our synthesized NPs showed higher selectivity for isomer product with $\sim 85 \%$ which didn't change much from the first cycle.

Figure 4.12. shows the recyclability of various Pd \& Pd-alloy NPs using ethyl acetate (EtOAc), chlorobenzene (Clben) and dichloromethane (DCM) at different hydrogen flow rates with $100 \%$ completion of the reaction. It shows that the recyclability of Pd NPs increased with addition of another electronegative metal to form the alloy as it imparts stability and reactivity to the overall NPs but not product selectivity which remained almost the same for all Pd-alloy NPs. The recyclability of (75:25) Pd:Au-Glu (Inert) NPs shows $100 \%$ completion of the catalytic reaction for at least 9 times with dichloromethane at $8.0 \pm 0.2 \mathrm{~mL} / \mathrm{min}$ hydrogen flow rate showing the GC-FID spectra for aqueous and organic phases and 100\% completion for at least 4 times with Ethyl acetate and only 3 times with chlorobenzene at $19.9 \pm 0.7 \mathrm{~mL} / \mathrm{min}$ hydrogen flow rate, all with the selectivity of more than $90 \%$ for 1-propanal (isomerization Product) under biphasic solvent system. We believe that the solvent like dichloromethane with the greatest solubility for allyl alcohol allows most cycles because the NPs are most stable in that solvent. Wu and co-workers ${ }^{145}$ reported that both $\mathrm{Pd} / \mathrm{C}$ and $\mathrm{Pd}$ immobilized on collagen fiber (CF) support exhibited a sharp loss in catalytic activity for hydrogenation of allyl alcohol after 5 


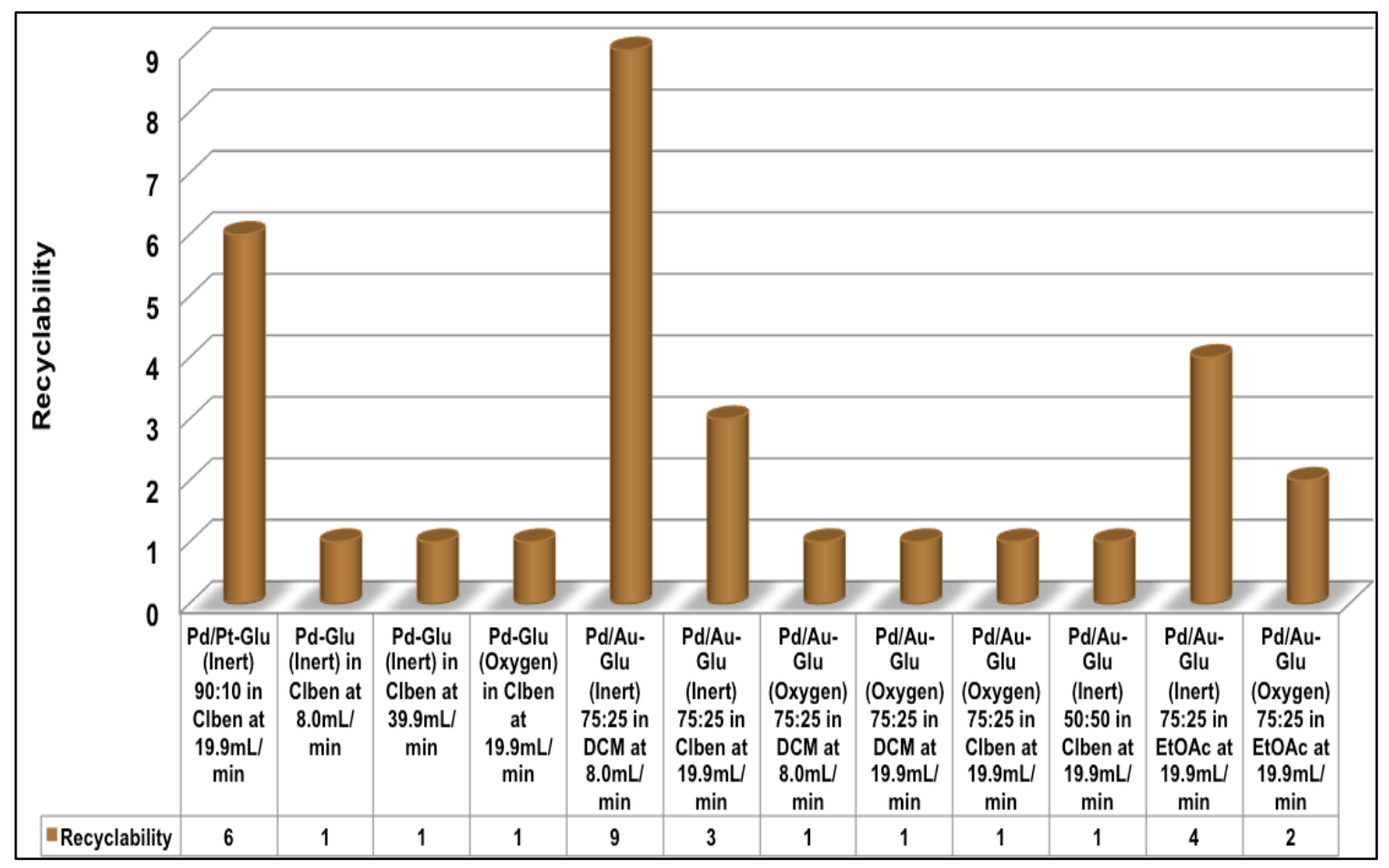

Figure 4.12. Plot showing the recyclability of various $\mathrm{Pd} \& \mathrm{Pd}$-alloy nanoparticles using ethyl acetate (EtOAc), chlorobenzene (Clben) and dichloromethane (DCM) at different hydrogen flow rates with $100 \%$ completion of the reaction. 
cycles from $75 \%$ to $15 \%$ with an extensive leaching of Pd from the catalyst to the reaction solution. In our synthesized (75:25) Pd:Au-Glu (Inert) NPs, they were quite stable as homogeneous catalyst even after 12 cycles with only slightly decrease in their catalytic activity as shown in Figure 4.11.

\subsubsection{Recycling stability of glutathione-coated PdAu NPs in various organic solvents}

We also compared the recycling stability of (75:25) Pd:Au-Glu (Inert) NPs in various organic solvents (chlorobenzene, ethyl acetate and dichloromethane) in biphasic condition with aqueous phase at $19.9 \pm 0.7 \mathrm{~mL} / \mathrm{min} \mathrm{H}_{2}$ flow rate using UVVis spectroscopy as shown in Figure 4.13. The UV-Vis spectra for NPs with ethyl acetate showed recycling stability for all 7 cycles with allyl alcohol, while with dichloromethane, NPs showed recycling stability for 5 cycles with allyl alcohol. With chlorobenzene, NPs showed recycling stability for 3 cycles. Every cycle ran for 30 minutes. The glutathione-coated Pd and Pd-alloy NPs are most stable in the presence of ethyl acetate in a biphasic solvent mixture.

\subsubsection{Recycling stability comparison between Pd-Glu (Inert) and Pd-Glu (Oxy) NPs}

We also compared the recycling stability of Pd-Glu (Inert) and Pd-Glu (Oxy) NPs for at least 6 cycles of hydrogenation/isomerization of allyl alcohol in chlorobenzene and nanopure water biphasic solvent system by UV-Vis spectroscopy as shown in Figure 4.14. The data clearly indicate that Pd-Glu (Inert) NPs were 

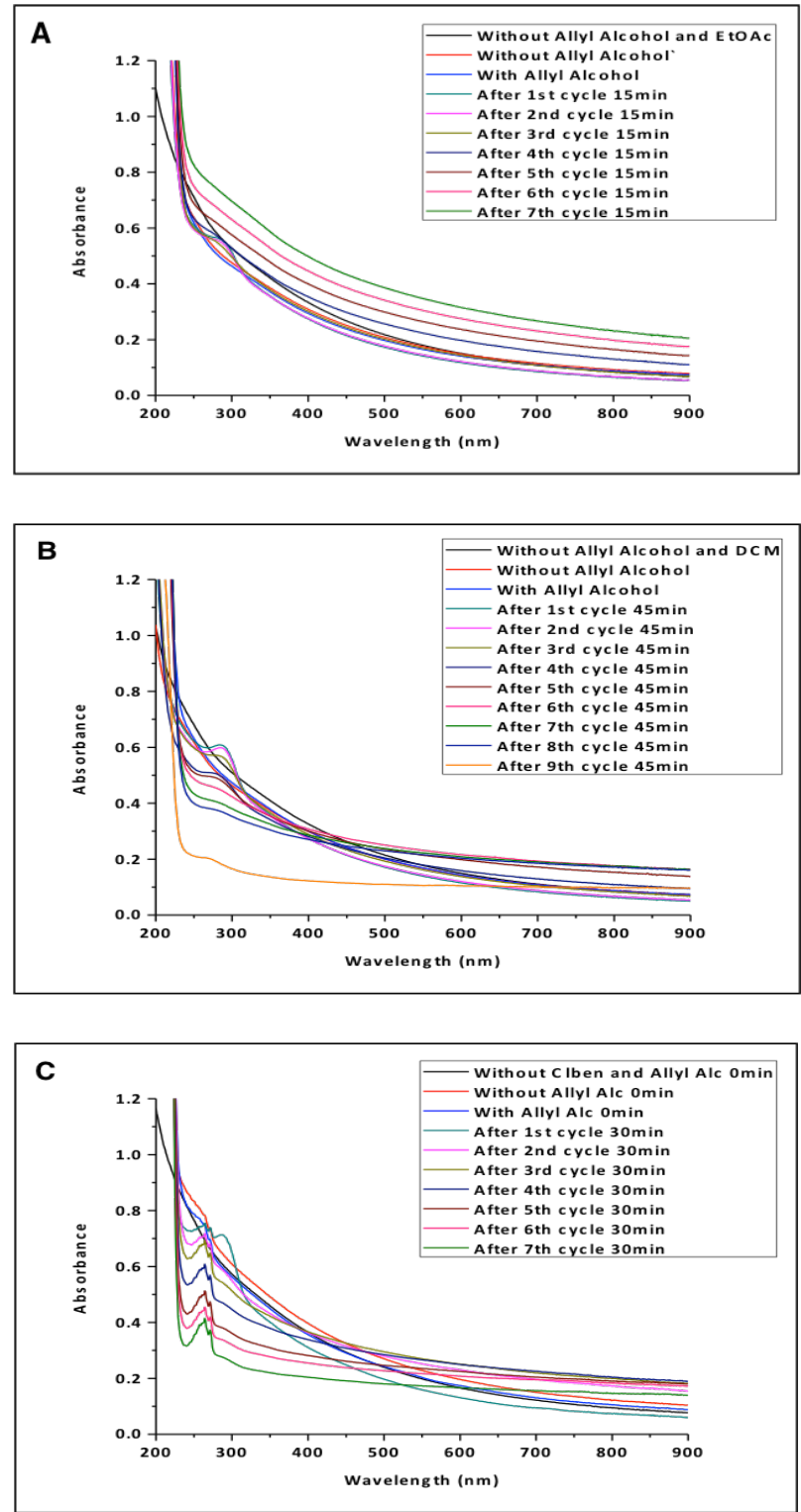

Figure 4.13. UV-Vis spectra comparing the recycling stability of (75:25) Pd:Au-Glu (Inert) NPs in various organic solvents [(A) ethyl acetate (stable for all 7 cycles), (B) dichloromethane (Stable for 5 cycles), and (C) chlorobenzene (Stable for 3 cycles)] with aqueous phase as the biphasic solvent system for 30 minutes each in the presence of allyl alcohol at $19.9 \pm 0.7 \mathrm{~mL} / \mathrm{min}$ hydrogen flow rate. 
stable for more cycles than Pd-Glu (Oxy) NPs since they were covered with more glutathione ligands than Pd-Glu (Oxy) NPs according to their TGA analysis, giving higher stability during many recycles.

\subsubsection{Comparison between Inert and Oxygen Pd:Au-Glu NPs}

Another important observation showed that (75:25) Pd:Au-Glu (Oxy) NPs have high TOF but low recyclability with $100 \%$ conversion rate when ethyl acetate solvent was used whereas, (75:25) Pd:Au-Glu (Inert) NPs have comparatively lower TOF but higher recyclability with same organic solvent as shown in Table 4.1 Similar

observations were seen for same NPs with chlorobenzene and dichloromethane solvents also. This shows that Pd NPs when synthesized under oxygen conditions have higher TOFs but low stability leading to low recyclability whereas Pd NPs synthesized under inert conditions have low TOFs but are more stable and shows higher recyclability. The TOF and stability are often a trade off.

\subsubsection{Biphasic catalysis for larger chain allylic alcohol substrates}

We continued with the use of (75:25) Pd:Au-Glu (Oxy) NPs as a pseudohomogeneous catalyst for the hydrogenation/isomerization of branched allyl alcohol that differ only slightly in chemical structure in the biphasic solvent mixture. For that purpose, we used 1-penten-3-ol (Figure 4.15.) and 3-buten-2-ol (Figure 4.16.) as substrates. The catalytic reaction of (75:25) Pd:Au-Glu (Oxy) NPs with 1penten-3-ol in ethyl acetate:water biphasic solvent mixture resulted in TOF of 317 moles converted/moles Pd/hour. Also, hydrogenation/isomerization of 3-buten-2- 

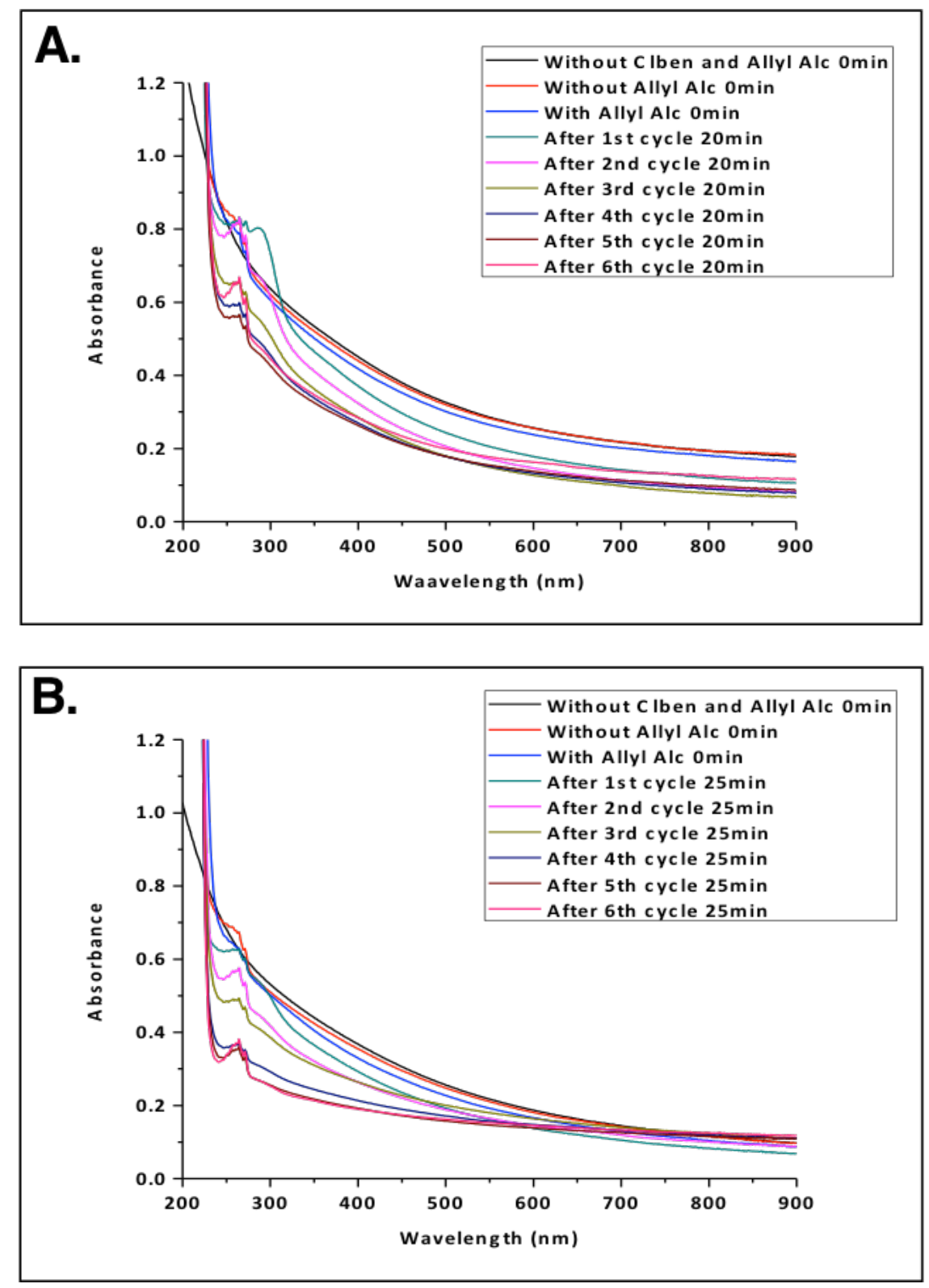

Figure 4.14. Recycling Stability of Pd-Glu (Inert) (above) and Pd-Glu (Oxyen) NPs (below) for 6 cycles of hydrogenation/isomerization of allyl alcohol in the presence of chlorobenzene/nanopure water biphasic solvent system by UV-Vis spectroscopy. 


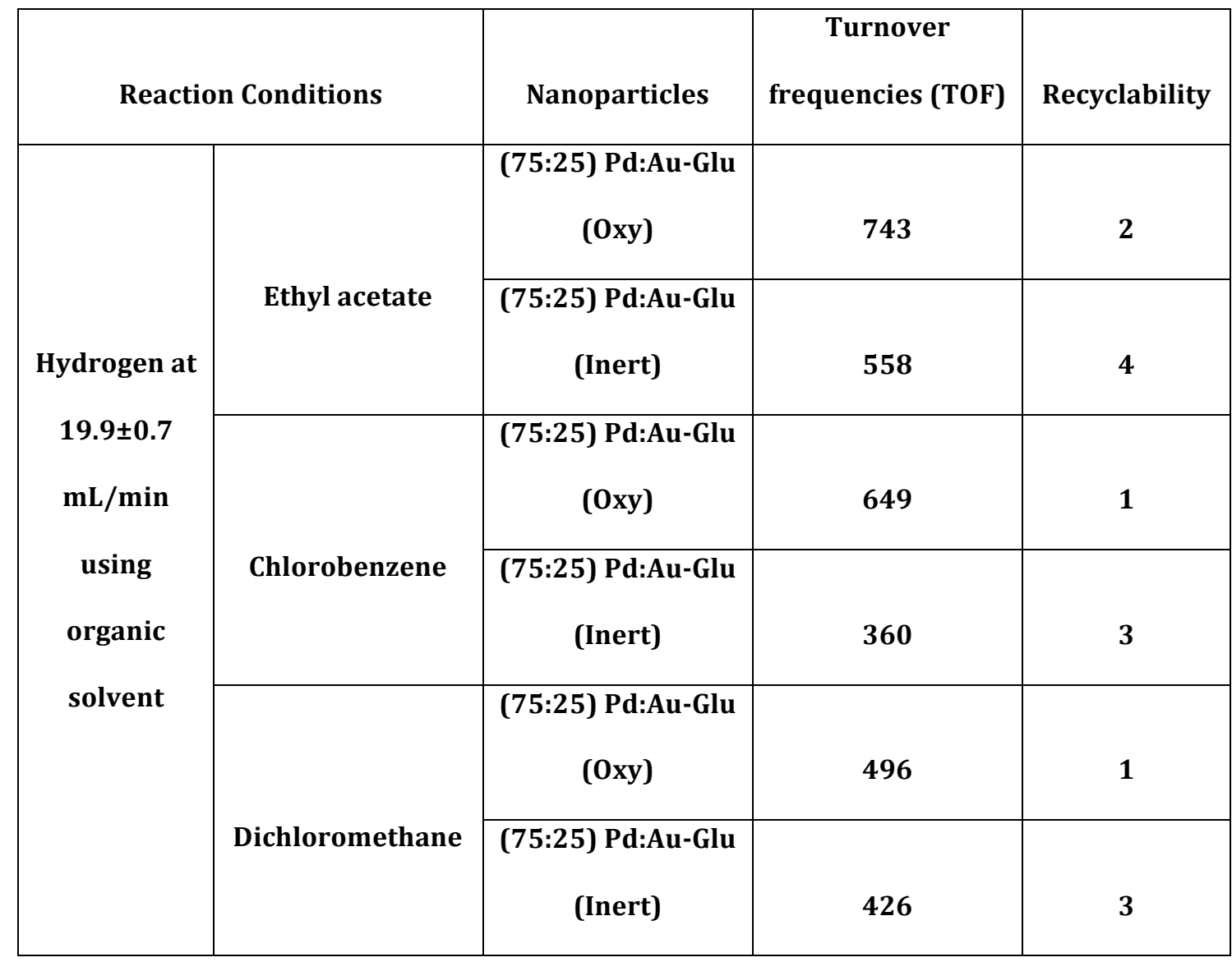

Table 4.1. Table showing turnover frequencies (TOF) and recyclability correlation for (75:25) Pd:Au-Glu (Inert/Oxygen) NPs in ethyl acetate, chlorobenzene and dichloromethane organic solvents in biphasic solvent mixture. 


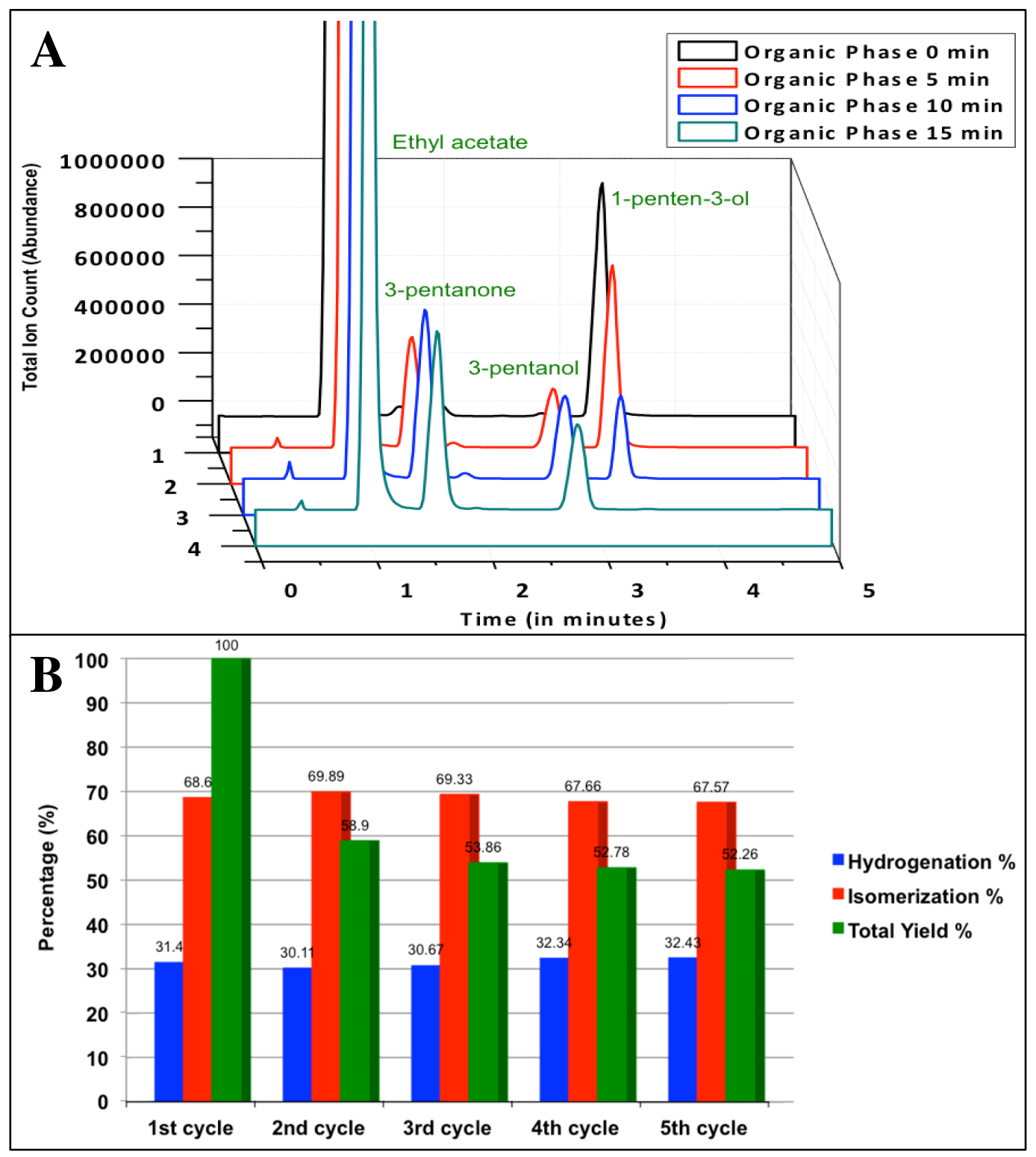

Figure 4.15. Gas chromatograms of organic layer (A) of a biphasic solvent mixture of $2 \mathrm{~mL}$ nanopure water and $2 \mathrm{~mL}$ ethyl acetate showing progress of the catalytic reaction of 1-penten-3-ol before and after exposure to $100 \%$ hydrogen (19.9 $\pm 0.7 \mathrm{~mL} / \mathrm{min}$ ) at different reaction times from $0,5,10$ and 15 minutes using $~ 6.0 \mathrm{mg}$ of (75:25) Pd:Au-Glu (Oxy) NPs. Frame (B) shows the recyclability of (75:25) Pd:Au-Glu (Oxy) NPs for 1-penten-3-ol up to 5 cycles showing \% hydrogenation, \% isomerization and \% total yield. 


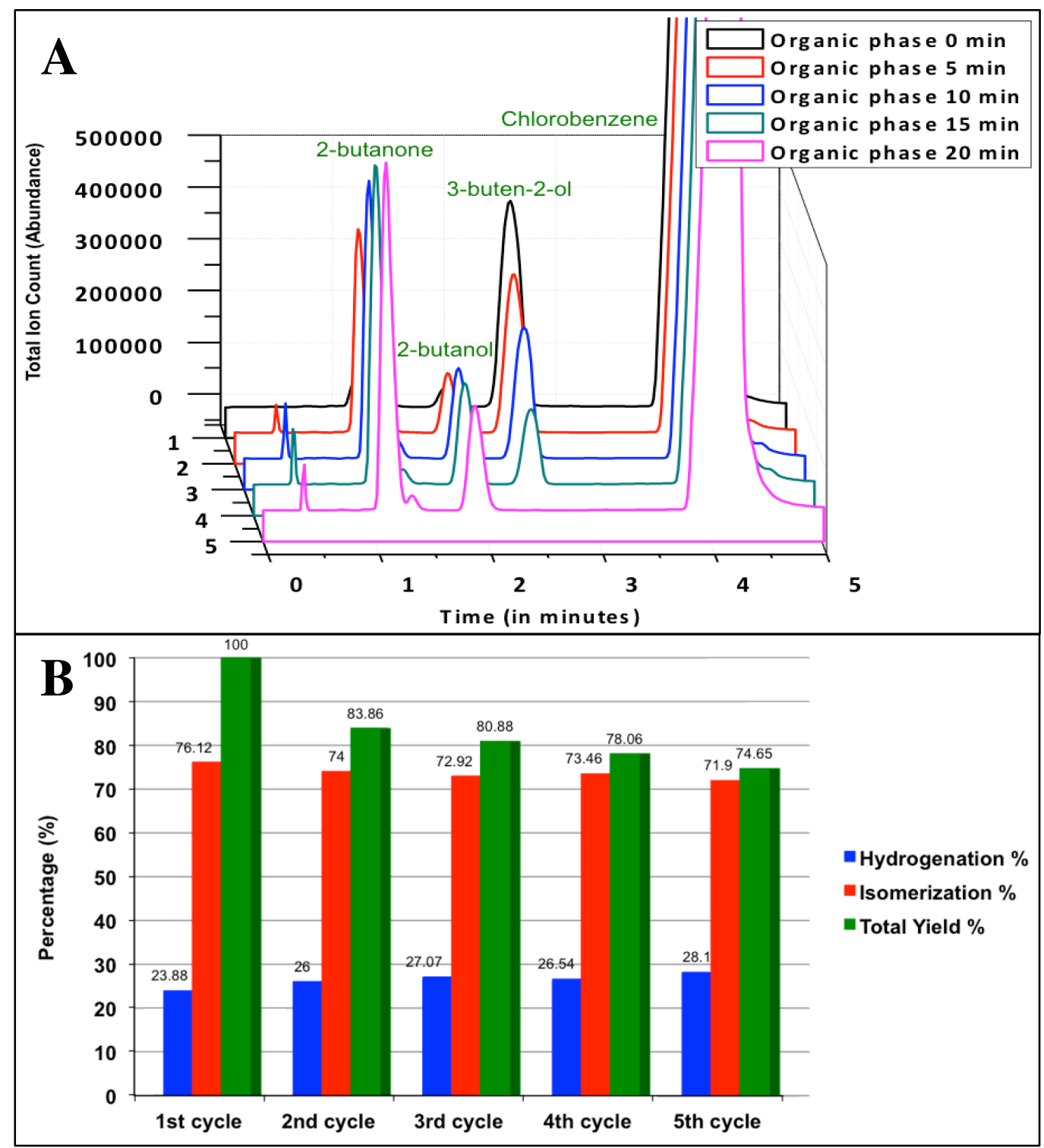

Figure 4.16. Gas chromatograms of organic layer (A) of a biphasic solvent mixture of $2 \mathrm{~mL}$ nanopure water and $2 \mathrm{~mL}$ chlorobenzene showing the progress of the catalytic reaction of 3-buten-2-ol before and after exposure to $100 \%$ hydrogen $(19.9 \pm 0.7 \mathrm{~mL} / \mathrm{min})$ at different reaction times from 0, 5, 10 and 15 minutes using $~ 6.0 \mathrm{mg}$ of (75:25) Pd:Au-Glu (Oxy) NPs. Frame (B) shows the recyclability of (75:25) Pd:Au-Glu (Oxy) NPs for 3-buten-2-ol up to 5 cycles showing \% hydrogenation, \% isomerization and \% total yield. 
ol using the same (75:25) Pd:Au-Glu (Oxy) NPs with chlorobenzene:water biphasic solvent mixture resulted in almost similar TOF of 340 moles converted/moles Pd/hour. These TOFs are lower than the TOF obtained from the same catalyst (75:25) Pd:Au-Glu (Oxy) NPs when used for catalyzing allyl alcohol.

Due to the hydrophobic nature, no products or reactant went to the aqueous phase. The recyclability for 1-penten-3-ol running each cycle for 20 minutes showed one complete cycle and then, it decreased to nearly $52 \%$ after 5 cycles, whereas the selectivity remains the same for 3-pentanone:3-pentanol (7:3) for all 5 cycles. Thus, in both cases, the NPs were more selective towards the isomer product even after 5 cycles. The recyclability for 3-buten-2-ol running each cycle for 25 minutes also showed one complete cycle and then it decreased to nearly $75 \%$ after 5 cycles, with selectivity for 2-butanone:2-butanol (7.5:2.5) for all 5 cycles. The Pd NPs underwent some aggregation after first cycle, but the selectivity of the catalyst basically remained unchanged over all cycles tested. Thus, we concluded that the larger substrates with longer hydrocarbon chain have lower reaction rates, likely due to restricted diffusion, as they are less soluble in aqueous phase and thus less access to the Pd through the glutathione ligands surrounding the Pd core.

The catalytic activity of 2 years old synthesized (75:25) Pd:Au-Glu (Inert) NPs were compared with newly synthesized (75:25) Pd:Au-Glu (Inert) NPs for hydrogenation/isomerization of allyl alcohol at $19.9 \pm 0.7 \mathrm{~mL} / \mathrm{min}$ hydrogen flowrate using ethyl acetate-aqueous biphasic solvent mixture as shown in Figure 4.17. The reactant was converted into products within 10 minutes as observed before 


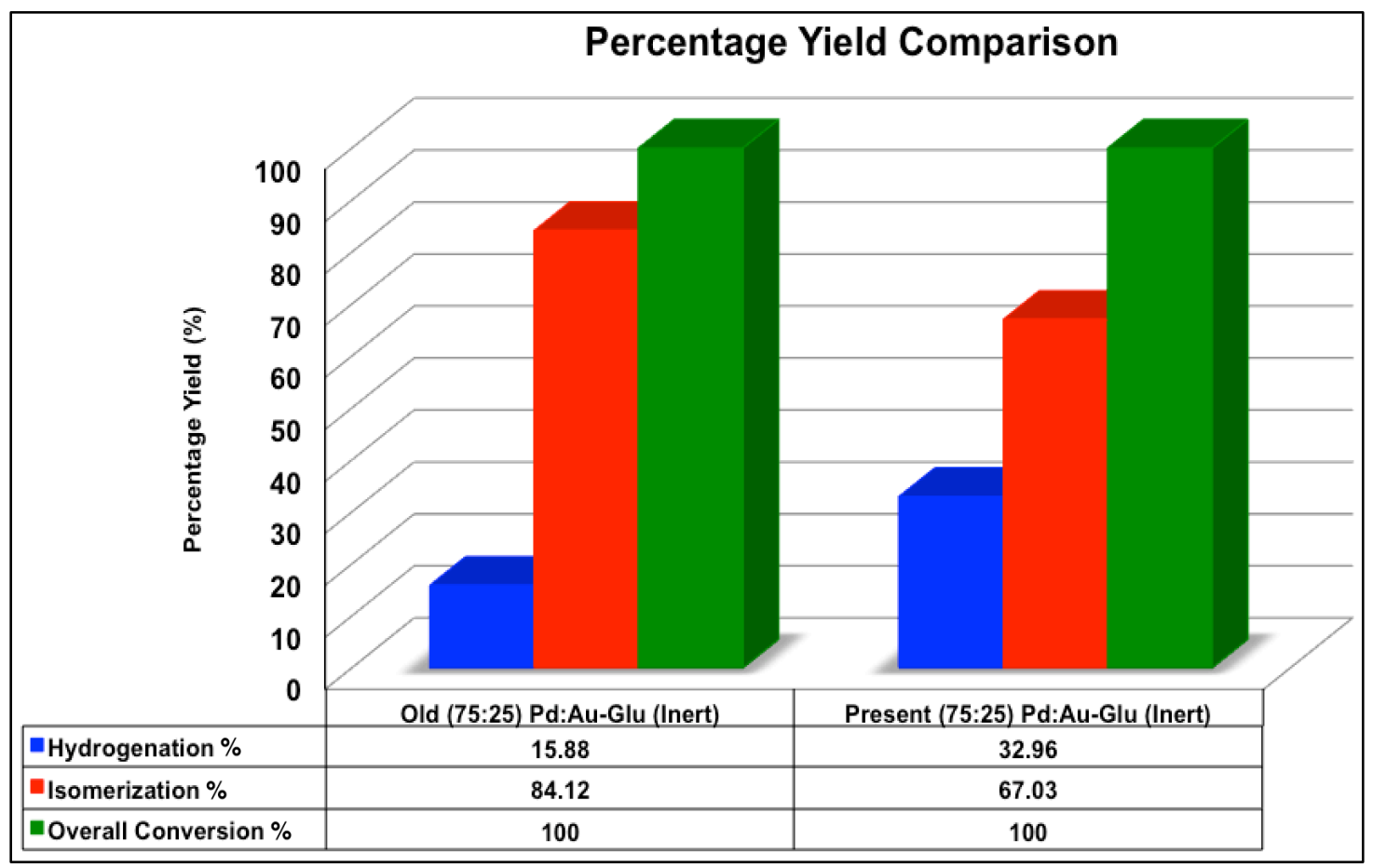

Figure 4.17. Plot showing catalytic activity comparison of 2 years old synthesized (75:25) Pd:Au-Glu (Inert) NPs versus newly synthesized (75:25) Pd:Au-Glu (Inert) NPs for hydrogenation/isomerization of allyl alcohol in terms of hydrogenation $\%$, isomerization $\%$ and overall conversion $\%$ at 19.9 $\pm 0.7 \mathrm{~mL} / \mathrm{min}$ hydrogen flow-rate using ethyl acetate-aqueous biphasic solvent mixture. 
which shows high stability of glutathione-coated NPs. The only difference observed was that $\%$ hydrogenation for 1-propanol got increased from $16 \%$ to $33 \%$ whereas $\%$ isomerization for 1-propanal decreased from $84 \%$ to $67 \%$, which might be due to the loss of some ligands from the Pd surface with time.

\subsubsection{Comparison with other Nanoparticles Catalysts}

Shon and co-workers reported the selectivity for the isomer product in the hydrogenation/isomerization of allyl alcohol using dodecanethiolate-capped Pd NPs generated from thiosulfate where the reaction was $80 \%$ complete after one hour. ${ }^{80}$, 81 Bruening and co-workers reported the TOFs using a common homogeneous catalyst, Wilkinson's catalyst, for the hydrogenation of allyl alcohol ${ }^{89}$ and showed the minor selectivity towards the formation of isomer of allyl alcohol. In a similar fashion, the rates of reaction with our synthesized NPs coated with glutathione compares very well with Wilkinson's catalyst with even better selectivity of up to 95\% for the isomerized product. Zharmagambetova and co-workers reported the selectivity of $74 \%$ for isomer product by using Pd-polymer complexes containing iminodithiol as catalysts. ${ }^{92}$ Our synthesized catalyst although have been stabilized with thiol bonded ligands which are known to decrease the catalytic activity due to strong Pd-thiol bond, ${ }^{75}$ still they exhibit higher catalytic activity (TOF=772) with (50:50) Pd:Au-Glu (Oxy) NPs being the best, as compared to other homogeneous catalytic system including Pd and Pt NPs stabilized by dendrimers ${ }^{51,93}$ and alkanethiolate monolayer (TOF < 500). ${ }^{34,} 80,81,132,133$ Other studies used collagen 
fiber coated with Pd NPs and hollow polystyrene nanospheres to catalyze the hydrogenation of allyl alcohol.

A few groups have also used bimetallic NPs like PdAu, PdPt and AgPd to catalyze the hydrogenation of allyl alcohol. For example, Scott and co-workers determined that the presence of $\mathrm{Ag}$ on AgPd NP catalyst prepared by galvanic exchange enhances the overall rate of hydrogenation over isomerization of allyl alcohol. ${ }^{183}$ Crooks and co-workers showed that Pd-Pt dendrimer -encapsulated NPs have higher catalytic activity than dendrimer-encapsulated Pd or Pt NPs for hydrogenation of allyl alcohol. ${ }^{96}$

\subsection{CONCLUSIONS}

In summary, we have reported the successful synthesis and stabilization of various water-soluble Pd and Pd-alloy NPs coated with glutathione which shows highly efficient catalytic performances with high TOFs, selectivity and recyclability based on the ratio of alloy-mixture composition, synthetic environment (inert or oxygen), organic solvent used in biphasic system and the hydrogen flow rates for hydrogenation/isomerization of allyl alcohols. We believe the lattice constant may play a role in the catalytic activity of the NPs resulting in oxygen synthesized NPs being more catalytically active than the inert ones. The ligands attached on the surface of the NPs play a bigger role than the different lattice constant for product selectivity. The catalytic activity of various glutathione-coated NPs showed that they followed the trend of lower activity near to monometal composition and more activity as the ratios of alloy metal to Pd increases. Pd:Au-Glu (Inert/Oxy) NPs have 
better TOFs than Pd:Pt-Glu (Inert/Oxy) NPs with same ratios, which are greater than pure Pd NPs. The electronegativity of Au and Pt metal are known to make Pd more electropositive improving substrate binding. Also, oxygen synthesized NPs being more catalytically active than the inert NPs. The selectivity towards hydrogenated and isomerized products after 1 complete cycle of reaction between allyl alcohol and $100 \%$ hydrogen at $19.9 \pm 0.7 \mathrm{~mL} / \mathrm{min}$ flow rate using chlorobenzene over glutathione coated Pd NPs suggested that NPs without alloying with other metals shows more selectivity towards isomerized product as compared to alloy NPs. For monometal Pd-Glu NPs, the selectivity remained the same for isomerized product even if the hydrogen flow rate increased from $8.0 \pm 0.3$ to $19.9 \pm 0.7$ to $39.9 \pm 0.2 \mathrm{~mL} / \mathrm{min}$. The ethyl acetate solvent in hydrogenation/isomerization reactions showed better TOFs than chlorobenzene and dichloromethane, likely due to high stability related to substrate solubility. The biphasic catalysis with chlorobenzene showed higher TOFs when Pd-Glu (Oxy) NPs were used but lower TOFs when Pd-Glu (Inert) NPs were used, as compared to using dichloromethane as the organic phase. These studies, in general, will help us to better understand the monolayer ligand chemistry, importance of lattice parameter of metal core and their utilization in designing better catalysts with high stability, catalytic activity and recyclability by tuning the ligand structure, metal compositions and using different flow rates of hydrogen in catalysis. It will also pave new avenues in the development of choosing better candidate for hydrogen sensing and storage. Taken together, this study will have a great impact in the field of catalysis and sensing. 


\title{
CHAPTER V
}

\author{
CATALYSIS STUDIES OF CITRATE-STABILIZED Pd AND CuPd CORE-SHELL \\ NANOPARTICLES FOR HYDROGENATION/ISOMERIZATION OF VARIOUS ALLYLIC
}

\section{ALCOHOLS}

\subsection{INTRODUCTION}

Here, we study the same hydrogenation and isomerization reactions of various allylic alcohols with increasing chain-length using a biphasic solvent system, but in the presence of citrate-stabilized Pd, PdAu and CuPd core-shell NPs to investigate their catalytic properties. As illustrated in Figure 5.1, the reactions between various allylic alcohols and hydrogen gas using $\mathrm{Pd}$ catalysts results in hydrogenation of the double bond to give the hydrogenated product and/or it may lead to migration of the double bond in keto-enol tautomerism fashion, yielding the corresponding saturated aldehyde. $152-154,159,180$ An important challenge is to increase the catalytic activity of the homogeneous catalysts while keeping them stable for reuse in several syntheses. We decided to study electrostatically stabilized, water-soluble citrate-coated Pd NPs as catalysts to be used in the aqueous phase of the biphasic solvent. Our hypothesis was that the activity would be much higher than the Pd-Glu NPs due to weak binding of the citrate, but that 


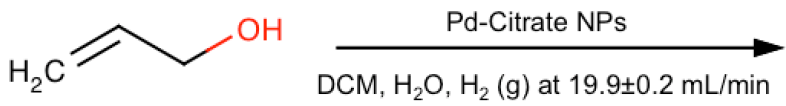

Allyl Alcohol

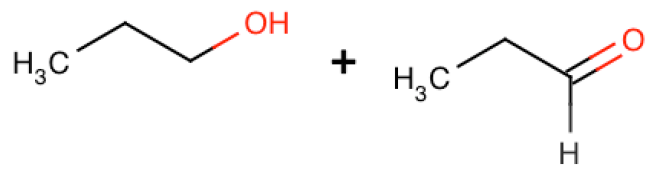

Propanal (Hydrogenation Pdt.) (Isomerization Pdt.)
$\left.\right|_{\mathrm{OH}} ^{\mathrm{CH}_{2} \mathrm{C}} \underset{\substack{\mathrm{DCM}, \mathrm{H}_{2} \mathrm{O}, \mathrm{H}_{2}(\mathrm{~g}) \text { at } \\ 19.9 \pm 0.7 \mathrm{~mL} / \mathrm{min}}}{\mathrm{Pd} \text {-citrate NPs }}$

1-penten-3-ol

(Reactant)<smiles>CCC(=O)CC</smiles>

3-pentanol (Hydrogenation Pdt.)

3-pentanone (Isomerization Pdt.)<smiles>C=CC(O)CCCC</smiles>
$\overrightarrow{\mathrm{DCM}, \mathrm{H}_{2} \mathrm{O}, \mathrm{H}_{2}(\mathrm{~g}) \text { at } 19.9 \pm 0.2 \mathrm{~mL} / \mathrm{min}}$

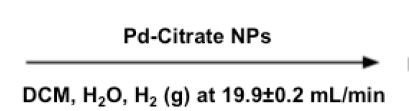<smiles>CCCC</smiles>

heptan-3-ol (Hydrogenation Pdt.) heptan-3-one (Isomerization Pdt.)<smiles>C=CC(C)(C)O</smiles>

2-methyl-3-buten-2-ol (Substrate)

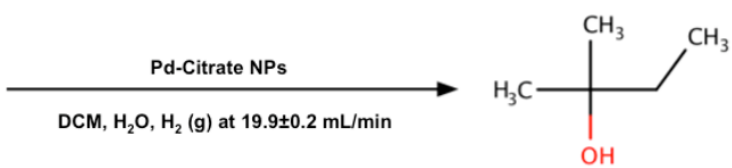

2-methylbutan-2-ol (Hydrogenation Pdt.)

Figure 5.1. Biphasic catalytic reactions showing the conversion of allyl alcohol, 1-penten-3-ol, 1-hepten-3-ol and 2-methyl-3-buten-2-ol to saturated alcohol (hydrogenated product) and/or aldehyde (isomerized product) in the presence of hydrogen and catalyst. 
stability could be a major issue for the same reason.

In this study, we describe the synthesis, stability and catalytic properties of Pd and CuPd core-shell NPs stabilized with citrate ligands as shown in Figure 5.2. Pd NPs were synthesized under inert nitrogen atmosphere and ice-cold temperature by sodium borohydride reduction using trisodium citrate as a stabilizer. Due to the weak affinity of the physisorbed citrate, ${ }^{179}$ the Pd surface is assumed to be mostly naked and available for substrate binding/activation. The catalytic performance of these water-soluble Pd-citrate NPs was compared for allyl alcohol, 1-penten-3-ol and 1-hepten-3-ol substrates.

\subsection{RESULTS AND DISCUSSION}

Using a biphasic solvent system in catalytic reactions is advantageous as the products can be easily removed from the organic phase and the catalyst dissolved in the aqueous phase can be reused several times without precipitating and redissolving. To the best of our knowledge, there has been no report regarding the utilization of citrate-stabilized Pd NPs as pseudo-homogeneous catalysts for the hydrogenation/isomerization of allylic alcohols.

\subsubsection{Biphasic hydrogenation/isomerization catalysis with citrate-coated NPs}

The catalytic reaction of $\alpha, \beta$-unsaturated alcohols (allyl alcohol, 1-penten-3ol, 1-hepten-3-ol or 2-methyl-3-buten-2-ol) to hydrogenated and/or isomerized product was performed by dissolving $2 \mathrm{~mL}$ of aqueous Pd-citrate nanoparticles solution in a $10 \mathrm{~mL}$ glass vial capped with a septum with an outlet for spent 


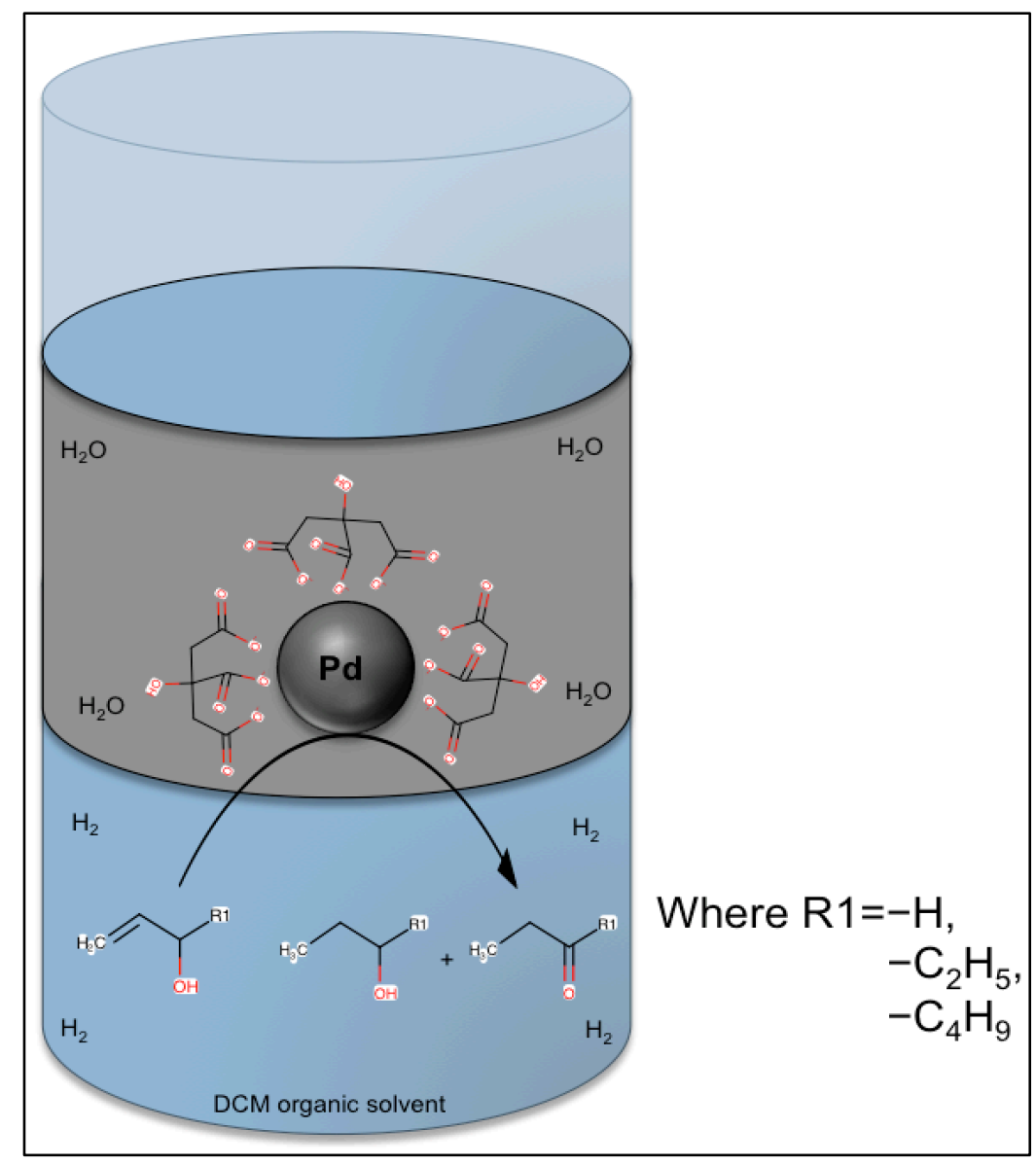

Figure 5.2. Schematic illustration of a biphasic catalytic reaction using $2 \mathrm{~mL}$ of dichloromethane (DCM) and $2 \mathrm{~mL}$ of nanopure water as solvent citratecoated Pd NPs as the catalyst and various chain-lengths of allyl alcohols (2propen-1-ol, 1-penten-3-ol, 1-hepten-3-ol or 2-methyl-3-buten-2-ol) as substrate. 
hydrogen gas passed through a glass pipette. Then, $2 \mathrm{~mL}$ of organic solvent [dichloromethane (DCM)] containing $0.74 \times 10^{-3}$ moles of unsaturated alcohol was added, forming a biphasic reaction solution mixture. The reaction mixture was then vigorously stirred at $600-700 \mathrm{rpm}$ at room temperature and under atmospheric pressure. Hydrogen gas was purged at a given flow rate $(19.9 \pm 0.7 \mathrm{~mL} / \mathrm{min})$ through a glass pipette and $20 \mu \mathrm{L}$ aliquots were removed from both aqueous and organic phases at particular intervals of time. The progress of the catalysis reaction was followed by gas chromatography (GC) of samples before exposure to hydrogen and after addition of catalyst and exposure to hydrogen at different intervals of time.

The catalytic reaction of $\alpha, \beta$-unsaturated alcohols (allyl alcohol, 1-penten-3ol, 1-hepten-3-ol or 2-methyl-3-buten-2-ol) to hydrogenated and/or isomerized product were originally performed by adding $2 \mathrm{~mL}$ of ethyl acetate or chlorobenzene in $2 \mathrm{~mL}$ of aqueous solution of Pd-citrate NPs. Pd-citrate NPs weren't stable in either of these two organic solvents and precipitated within 5 minutes of stirring inside the reaction vial as shown in Figure 5.3. The Pd-citrate NPs were shown to be quite stable in $2 \mathrm{~mL}$ dichloromethane- $2 \mathrm{~mL}$ aqueous biphasic solvent system even after 40 minutes, which was then used for all catalytic reactions under hydrogen gas purging. Figure 5.4. shows the GC spectra of the organic dichloromethane layer (Frame A) and of the aqueous (Frame B) at various times for the biphasic catalysis of $0.74 \times 10^{-3}$ moles of allyl alcohol dissolved in $2 \mathrm{~mL}$ of assynthesized aqueous Pd-citrate NPs solution and $2 \mathrm{~mL}$ dichloromethane. 

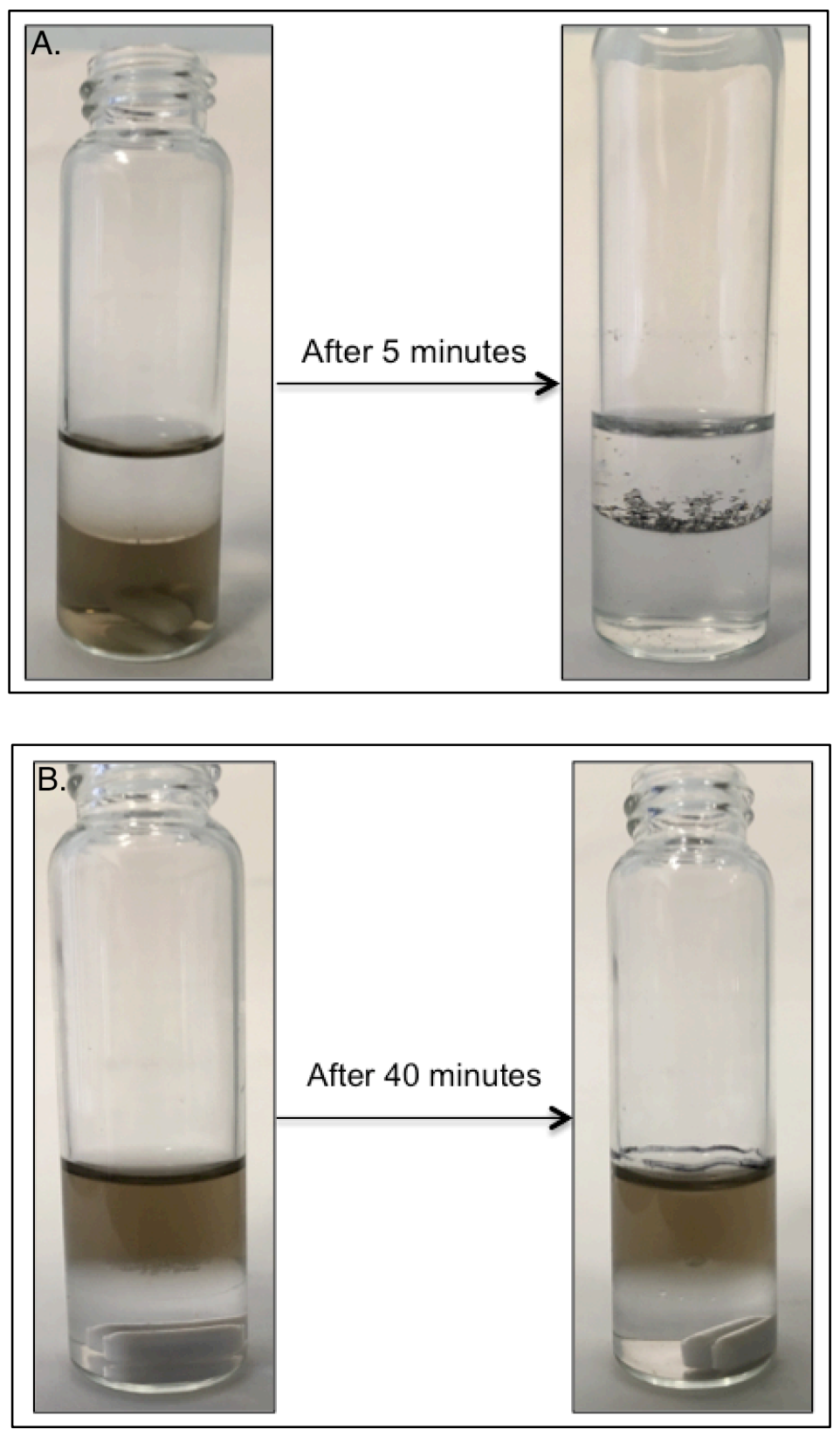

Figure 5.3. Stability of Pd-citrate NPs in the presence of $0.74 \times 10^{-3}$ moles of allyl alcohol at $19.9 \pm 0.7 \mathrm{~mL} / \mathrm{min}$ hydrogen flow rate in the biphasic solvent mixture of nanopure water and (A) ethyl acetate or chlorobenzene (not shown) after 5 minutes and (B) dichloromethane after 40 minutes. 

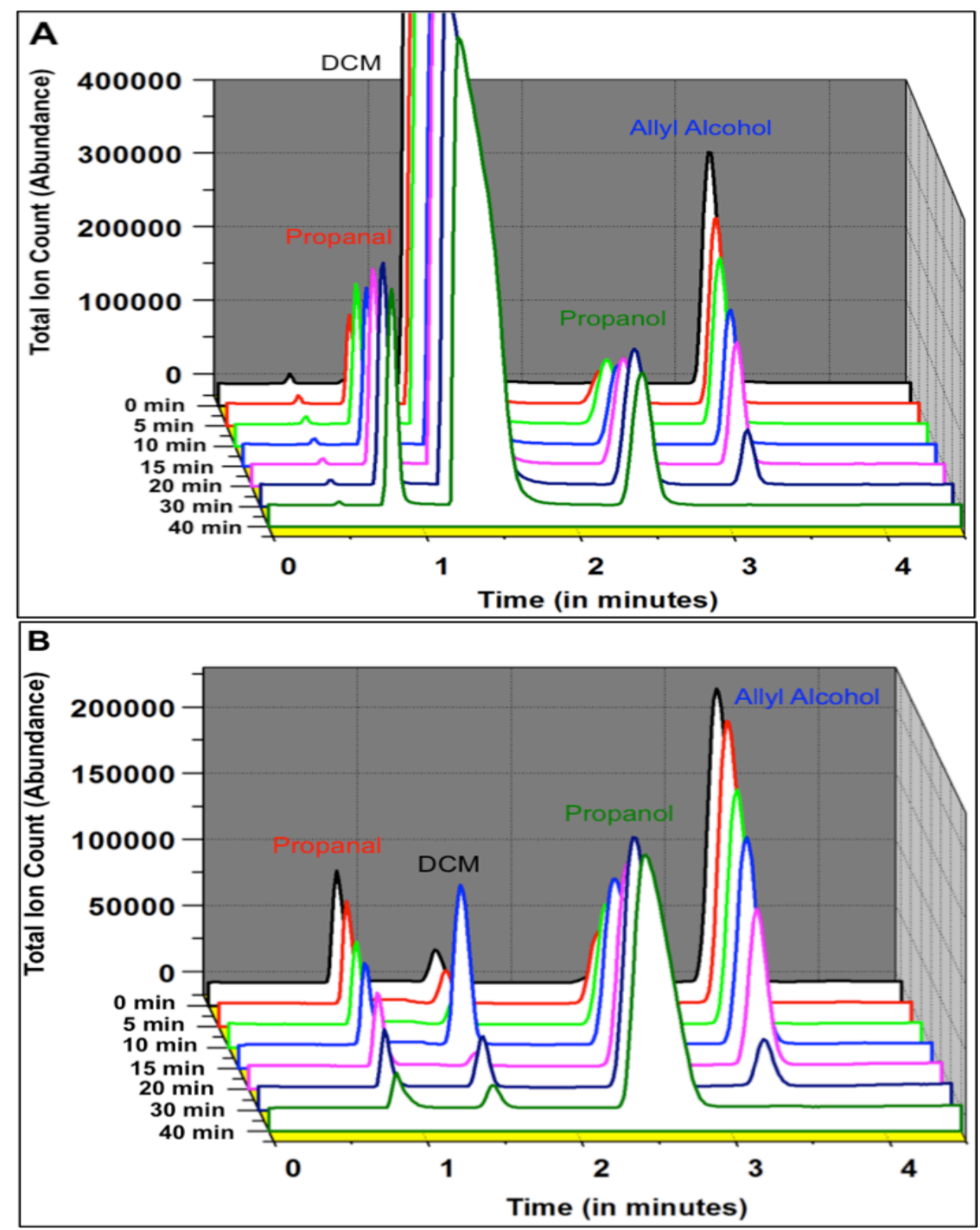

Figure 5.4. Example of biphasic catalysis for $0.74 \times 10^{-3}$ moles of allyl alcohol using $2 \mathrm{~mL}$ of aqueous Pd-citrate NPs solution and $2 \mathrm{~mL}$ dichloromethane solvent using $19.9 \pm 0.7 \mathrm{~mL} / \mathrm{min} \mathrm{H}_{2}$ flow rate for 40 minutes. GC-FID data for organic layer (A) and aqueous layer (B) a 0, 5, 10, 15, 20, 30 and 40 minutes reaction times with retention times for both reactant and products shown. 
In the aqueous phase, the peak corresponding to allyl alcohol coming at 3.30 minute decreased with time as expected for it reacting with hydrogen, whereas the peaks corresponding to propanal and 1-propanol at times 0.82 and 2.46 minutes, respectively, increased. In the organic phase, the peak heights for both products increased largely with time as the allyl alcohol peak height decreased and eventually vanished completely, showing the presence of both products at 40 minutes. The organic layer was separated from the aqueous layer and product trapped inside aqueous layer was further extracted by washing with $2 \mathrm{~mL}$ of dichloromethane twice to remove the remaining products. Thus, the Pd-citrate NP catalysts remained dissolved in the aqueous phase, which was recovered and reused several times.

\subsubsection{Turnover Frequencies (TOFs) of Pd catalysts.}

The turnover frequency (TOF) of the products were calculated using the slope of the linear portion of up to $>60 \%$ of the conversion of reactant to isomer and hydrogenated product in the plot of percentage hydrogenation and percentage isomerization versus time as reported previously in our lab and others. ${ }^{75,}{ }^{89}$ For example, Figure 5.5. shows the plot of \% hydrogenation and \% isomerization of $0.74 \times 10^{-3}$ moles of 1-hepten-3-ol versus reaction time using Pd-citrate NPs. in $2 \mathrm{~mL}$ nanopure water and $2 \mathrm{~mL}$ dichloromethane at $19.9 \pm 0.7 \mathrm{~mL} / \mathrm{min}$ flow rate of hydrogen and used for determining slope to calculate TOF when $>60 \%$ of 1-hepten3-ol was reacted to form both hydrogenated and isomerized products. 


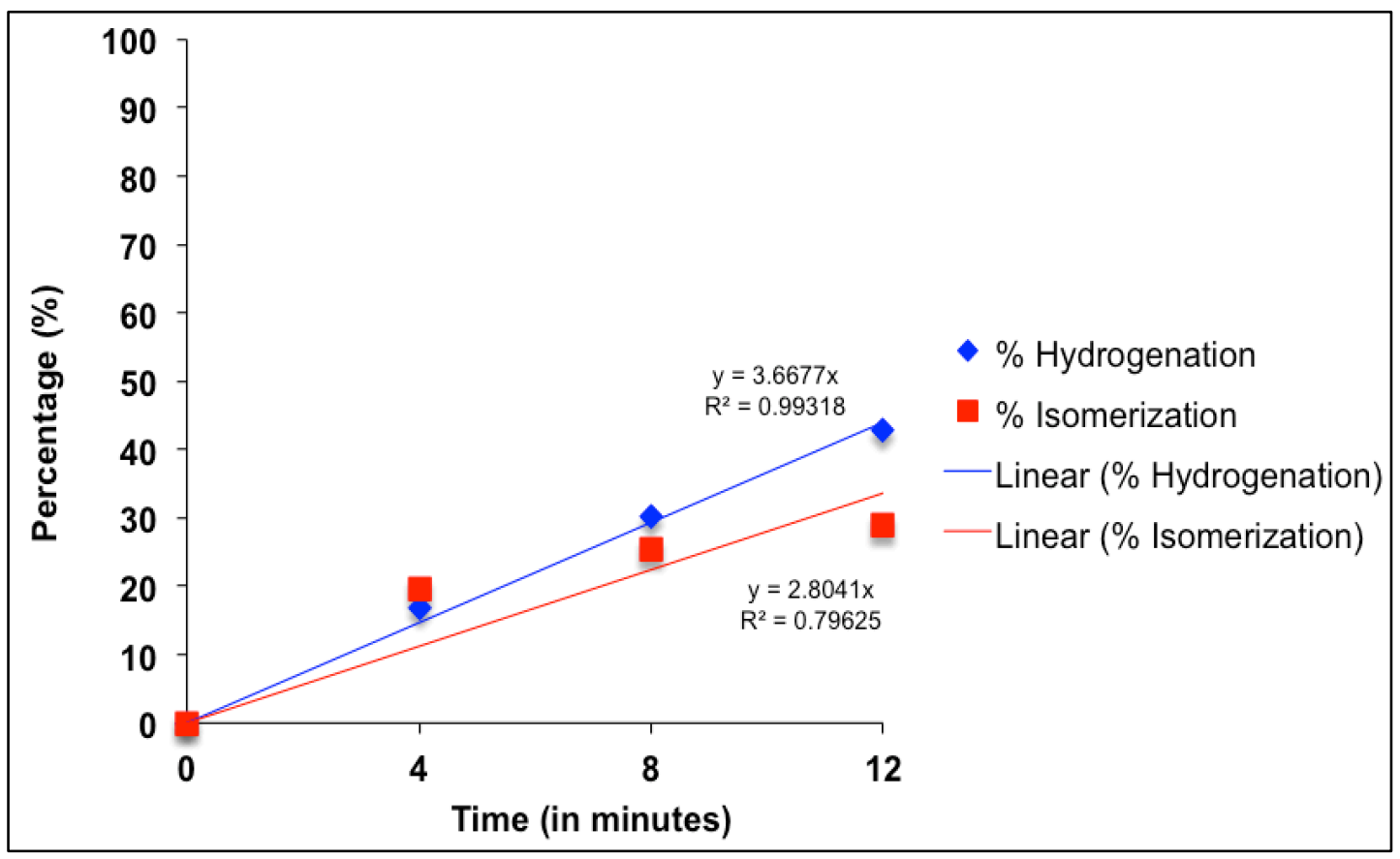

Figure 5.5. Example of calculating TOF using plot of $\%$ hydrogenation and $\%$ isomerization of $0.74 \times 10^{-3}$ moles of 1 -hepten-3-ol versus reaction time over $2 \mathrm{~mL}$ aqueous Pd-citrate NPs solution and $2 \mathrm{~mL}$ dichloromethane at $19.9 \pm 0.7$ $\mathrm{mL} / \mathrm{min}$ flow rate of hydrogen and used for determining slope to calculate TOF when $>60 \%$ of 1 -hepten-3-ol was reacted. 


\subsubsection{Catalytic Activity and Selectivity of Pd-citrate NP Catalysts}

Table 5.1. compares the catalytic performance of the Pd-citrate NPs for the hydrogenation/isomerization of the different substrates. Here, the substituent attached $\alpha$ to the double bond changes from $-\mathrm{H}$ to $-\mathrm{C}_{2} \mathrm{H}_{5}$ to $-\mathrm{C}_{4} \mathrm{H}_{9}$ for the different substrates, thus, increasing the chain-length of the substrate. It was observed that turnover frequency (TOF) increased with increasing chain-length, which was highest at 8870 moles product/moles Pd/hour for 1-penten-3-ol, whereas, 1hepten-3-ol shows a TOF of 6114 and allyl alcohol shows 4452 . This clearly shows that 1-penten-3-ol was the most highly reactive towards hydrogenation/ isomerization reaction in the presence of Pd-citrate NPs which we believe could be due to the presence of the intermediate alkyl chain that remained dissolved in the organic phase, orienting its double bond active site towards the aqueous phase in which Pd-citrate NPs are dissolved. This may lead to better reactivity to give the products. The shorter allyl alcohol substrate is more miscible in both the organic and aqueous phases due to the smaller size and may randomly orients itself towards the Pd NPs, which may not place it in the optimal position for reactivity, thus resulting in a lower TOF. In the case of 1-hepten-3-ol, we observed that the Pdcitrate NPs partitioned into the organic phase due to the high solubility of the longer alkyl chain-length of the substrate. Once the reaction has complete, the Pd-citrate NPs partitioned back to the aqueous phase. This was confirmed by the UV-Vis spectroscopy as shown in Figure 5.6, where $0.74 \times 10^{-3}$ moles of 1-hepten-3-ol was

used as substrate with Pd-citrate NPs in biphasic solvent mixture and the organic phase was monitored under UV-Vis spectroscopy for $0,4,12,20$ minutes. The 


\begin{tabular}{|cccccc|}
\hline \multirow{2}{*}{ Substrate } & \multicolumn{6}{c}{ Turnover Frequencies (TOFs, $\mathbf{h}^{-1}$ ) } & $\begin{array}{c}\text { Selectivity } \\
\text { (Hydrogenated: } \\
\text { Isomerized) }\end{array}$ & $\begin{array}{c}\text { Recyclability } \\
\text { (100\% yield) }\end{array}$ \\
\cline { 2 - 6 } & 2134 & 2318 & 4452 & $61: 39$ & 3 \\
\hline & 4990 & 3880 & 8870 & $58: 42$ & 5 \\
\hline
\end{tabular}

Table 5.1. Catalytic comparison (activity, selectivity and recyclability) between different structurally related $\alpha, \beta$-unsaturated alcohols including allyl alcohol, 1penten-3-ol, 1-hepten-3-ol and 2-methyl-3-buten-2-ol using biphasic solvent system of $2 \mathrm{~mL}$ of as-synthesized aqueous Pd-citrate NPs solution (Pd NPs $=0.15 \mathrm{mg}$ ) and 2 $\mathrm{mL}$ dichloromethane organic solvent at $19.9 \pm 0.7 \mathrm{~mL} / \mathrm{min}$ hydrogen flow rate. 

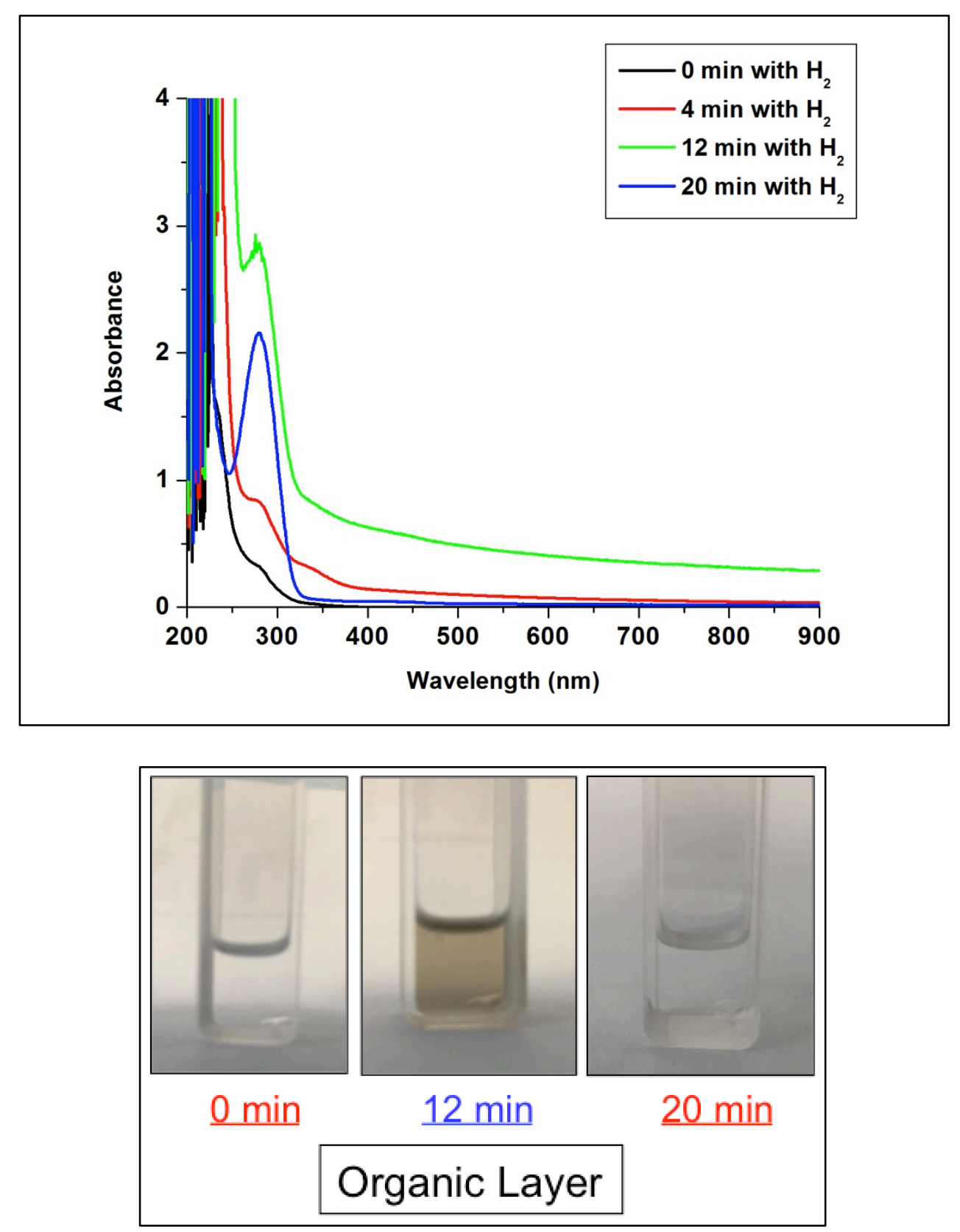

Figure 5.6. UV-Vis spectra showing lower stability of Pd-citrate NPs by migrating to the organic phase during the reaction with $0.74 \times 10^{-3}$ moles of 1 hepten-3-ol at 19.9 $\pm 0.7 \mathrm{~mL} / \mathrm{min}$ hydrogen flow rate under biphasic solvent system of $2 \mathrm{~mL}$ of as-synthesized aqueous Pd-citrate NPs solution (Pd NPs = $0.15 \mathrm{mg}$ ) and $2 \mathrm{~mL}$ dichloromethane organic solvent. 
absorbance at higher wavelength increased with the reaction time with maximum at 12 minutes which shows that Pd-citrate NPs partitioned to the organic phase and after the reaction completes at 20 minutes, the absorbance goes down at higher wavelength as Pd NPs partitioned back to the aqueous phase. Also, the color of the organic phase changes initially from colorless to brown and then, back to colorless confirming the NPs migration. This migration of NPs from aqueous to organic and back to aqueous caused lower stability of the Pd NPs and thus, its turnover frequency remained less than that of 1-penten-3-ol substrate. All three substrates showed slightly higher selectivity towards the hydrogenated product with ratios of 3:2 towards the hydrogenated:isomerized products. The TOF values for all of these substrates are incredibly large compared to those described in literature, which are generally below 500 .

\subsubsection{Procedure for recyclability of Pd-citrate NPs.}

In order to reduce the overall production cost of the final products using the catalyst, it is very important for the catalysts to be highly recyclable. The recyclability of citrate-coated Pd NPs using the biphasic solvent system of nanopure water and dichloromethane organic solvent that are immiscible to each other at 19.9 $\pm 0.7 \mathrm{~mL} / \mathrm{min}$ hydrogen flow rate were determined for allyl alcohol, 1-penten-3ol and 1-hepten-3-ol by reusing the same Pd NPs again after fully extracting the product from the aqueous phase with dichloromethane. This biphasic solvent system makes it very easy to separate the catalyst since the products easily partition 
into the organic phase. The aqueous catalyst can be easily reused for potentially several cycle.

Figure 5.7. shows the GC-FID spectra of the aqueous and organic phases for the recyclability of Pd-citrate NPs with $100 \%$ completion of the catalytic reaction for at least 5 times with 1-penten-3-ol at 16 minutes each, as NPs remained stable during those cycles. Allyl alcohol showed a recyclability of 3 times at 40 minutes each, and 1-hepten-3-ol showed 2 times at 20 minutes each, all with a selectivity of 3:2 for hydrogenated:isomerized products. Here, the GC-FID for the organic layer showed peaks corresponding to the products (hydrogenated and isomerized) after a particular interval of time, showing the complete conversion of unsaturated alcohol to products. The last cycle showed the presence of substrate remaining, showing an incomplete reaction. Similar results were observed in the aqueous phase. Each catalytic cycle involved the addition of $2 \mathrm{~mL}$ of as-synthesized aqueous Pd-citrate NP solution and $2 \mathrm{~mL}$ of dichloromethane organic solvent in a $10 \mathrm{~mL}$ reaction vial. $0.74 \times 10^{-3}$ moles of unsaturated alcohol were then injected into the reaction mixture and performed as before. This procedure was repeated for a number of cycles until product conversion with less than $100 \%$ yield was observed. The reaction was monitored by GC-FID. This biphasic solvent system makes it very easy to separate catalyst from the products, since products go into the organic phase and can be easily removed by simple separation from aqueous solution containing the catalyst. The catalyst dissolved in the aqueous phase can be easily reused for the next cycle. 

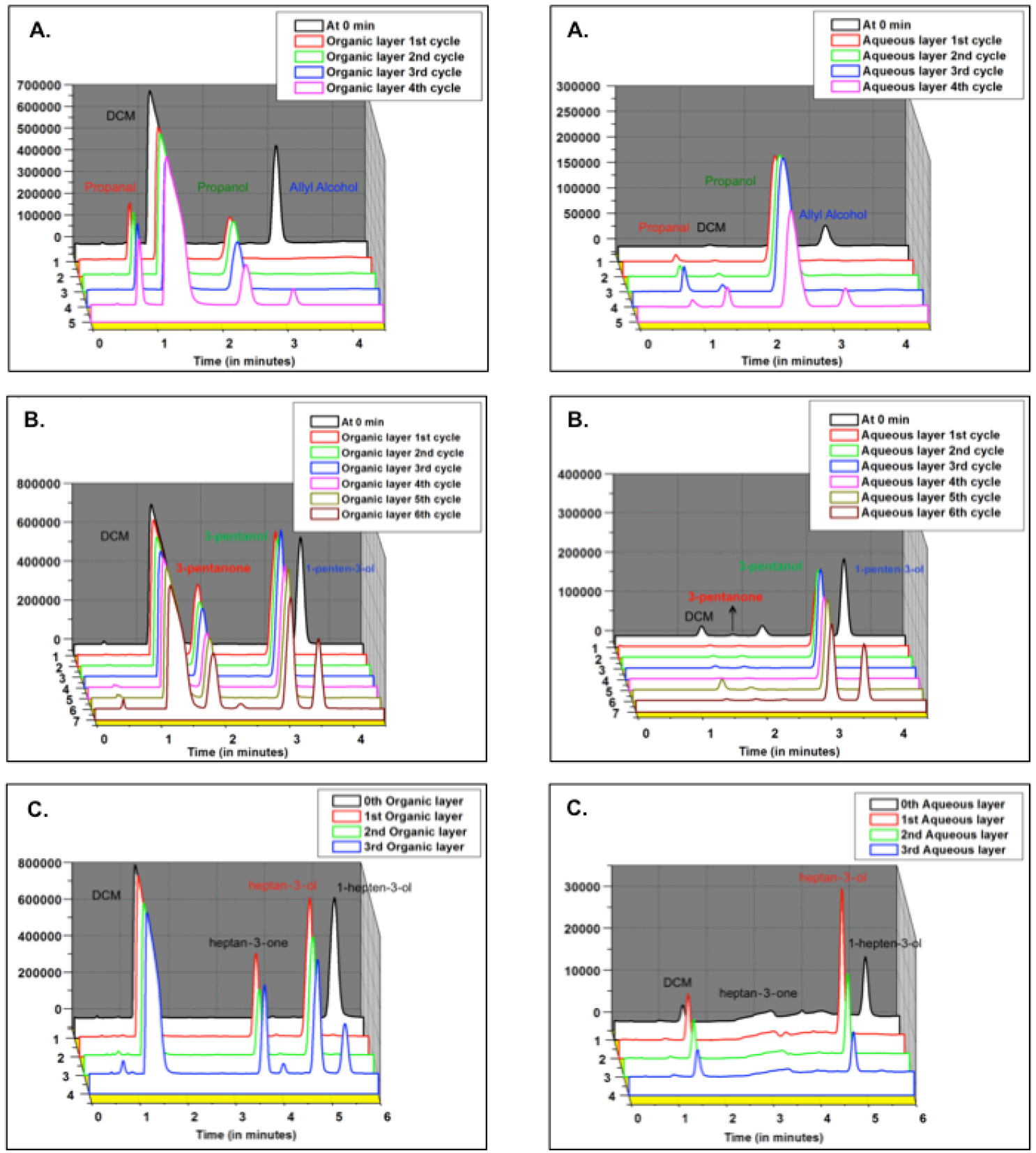

Figure 5.7. GC-FID spectra of aqueous and organic phases for the recyclability of Pd-citrate NPs showing 100\% completion of the catalytic reactions with $0.74 \times 10^{-3}$ moles of substrate for at least (A) 3 times for allyl alcohol, (B) 5 times for 1-penten-3-ol and (C) 2 times for 1-hepten-3-ol with dichloromethane at $19.9 \pm 0.7 \mathrm{~mL} / \mathrm{min}$ hydrogen flow rate. 


\subsubsection{Catalytic comparison between straight chain and branched substrates}

In order to understand the role of substrate alkyl chain in the variation of TOFs among different chain-length substrates, we compared the reactivity of 1penten-3-ol with the branched alkyl chain substrate 2-methyl-3-buten-2-ol having the same number of carbon atoms as shown in Table 5.2. Since the isomerized product wasn't possible with 2-methyl-3-buten-2-ol substrate, the hydrogenated TOF was observed to be the overall TOF of the reaction, which came out to be 4598 moles product/moles Pd/hour. This overall TOF was much lower than the TOF of 8870 for 1-penten-3-ol since 2-methyl-3-buten-2-ol could partition more in the aqueous phase as seen in the GC chromatography, thus destabilizing Pd-citrate NPs. This clearly provides evidence that the long alkyl chain helps for getting a better TOF with the same catalyst. Also, the Pd-citrate NPs were shown to be stable for 5 cycles using 1-penten-3-ol, but only 2 times for 2-methyl-3-buten-2-ol with $100 \%$ product conversion. Again, this shows that Pd-citrate NPs are more stable when a substrate with a long linear alkyl chain was used as compared to a branched alkyl chain with the same number of carbon atoms.

\subsubsection{Catalytic comparison between mono-phasic and biphasic solvent systems}

We also compared the TOF of hydrogenation/isomerization reaction of 1penten-3-ol under both mono-phasic and biphasic solvent systems in order to know the importance of the organic solvent in the catalytic reaction. For the mono-phasic condition, $2 \mathrm{~mL}$ of aqueous Pd-citrate NPs solution was combined with $0.74 \times 10^{-3}$ 


\begin{tabular}{|c|c|c|c|c|c|}
\hline \multirow{2}{*}{ Substrate } & \multicolumn{3}{|c|}{ Turnover Frequencies (TOF, $h^{-1}$ ) } & \multirow{2}{*}{$\begin{array}{c}\text { Selectivity } \\
\text { (Hydrogenated: } \\
\text { Isomerized) }\end{array}$} & \multirow[b]{2}{*}{$\begin{array}{l}\text { Recyclability } \\
\text { (100\% yield) }\end{array}$} \\
\hline & Hydrogenated & Isomerized & Total & & \\
\hline $\mathrm{H}_{2} \mathrm{C}^{\prime}$ & 4990 & 3880 & 8870 & $58: 42$ & 5 \\
\hline $\mathrm{H}_{3} \mathrm{C}-\left.\right|_{\mathrm{OH}} ^{\mathrm{CH}_{3}} / /$ & 4598 & $\mathbf{0}$ & 4598 & $100: 0$ & 2 \\
\hline
\end{tabular}

Table 5.2. Catalytic comparison (activity, selectivity and recyclability) between 1penten-3-ol and 2-methyl-3-buten-2-ol having same number of carbon atoms for hydrogenation/isomerization catalysis using Pd-citrate NPs at $19.9 \pm 0.7 \mathrm{~mL} / \mathrm{min}$ hydrogen flow rate using biphasic solvent system of dichloromethane and aqueous phase. 
moles of 1-penten-3-ol in water under a $19.9 \pm 0.7 \mathrm{~mL} / \mathrm{min}$ hydrogen flow rate. The catalytic activity over 60 minutes is shown in Figure 5.8. The substrate was fully converted into products after 30 minutes and the TOF for the reaction comes out to be 5978 moles product/moles Pd/hour (Hydrogenated TOF=3104, isomerized TOF=2875). The same Pd-citrate NPs as a catalyst and same 1-penten-3-ol as substrate in the presence of the biphasic solvent mixture of $2 \mathrm{~mL}$ of dichloromethane and $2 \mathrm{~mL}$ of nanopure water showed an 8870 moles product/moles Pd/hour. This difference in TOF shows that the dichloromethane organic solvent is very important with the aqueous phase as a biphasic solvent mixture for achieving the highest catalytic activities.

\subsubsection{Kinetic studies of catalysis using different allylic alcohols}

We also studied the total percentage completion of the reaction with time for allyl alcohol, 1-penten-3-ol and 1-hepten-3-ol using Pd-citrate NPs at 19.9 \pm 0.7 $\mathrm{mL} / \mathrm{min}$ hydrogen flow rates under dichloromethane and aqueous phase. Examining the early stages of the reaction showed that the initial reaction rate for 1-penten-3ol was high for the first 4 minutes, whereas the rate of reaction for 1-hepten-3-ol was low due to migration and instability of Pd-citrate NPs in the organic phase. The rate of reaction for allyl alcohol was also low from the beginning due to random orientation of the substrate active site towards the Pd surface taking 40 minutes for the reaction to complete as shown in Figure 5.9.

As a control experiment, we also studied the catalytic activity of $0.15 \mathrm{mg}$ of $\mathrm{PdCl}_{4}^{2-}$ salt in aqueous solution along with $0.74 \times 10^{-3}$ moles of allyl alcohol at 


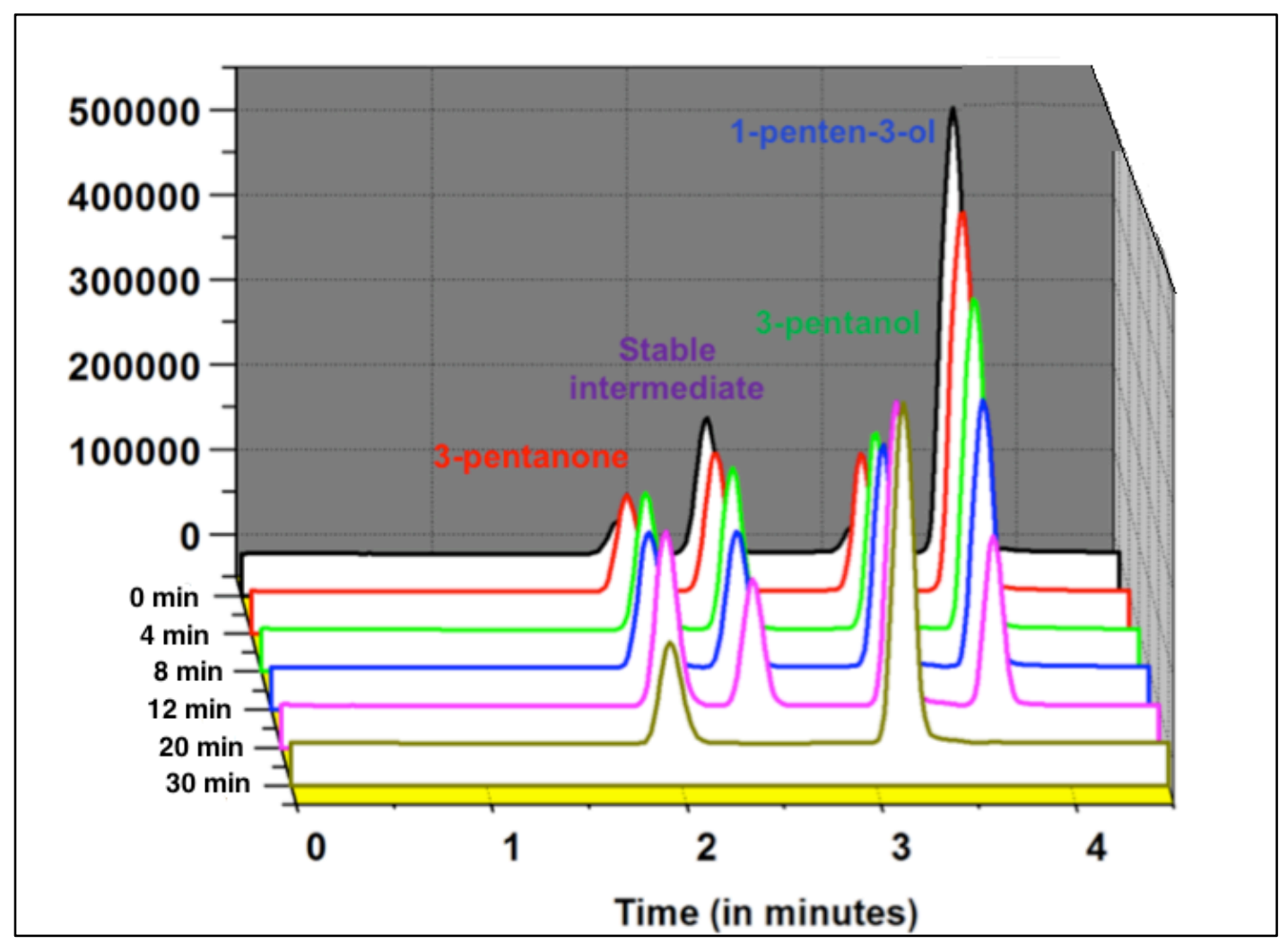

Figure 5.8. GC-FID spectra of mono-phasic hydrogenation/isomerization reaction using $0.74 \times 10^{-3}$ moles of 1 -penten-3-ol at $19.9 \pm 0.7 \mathrm{~mL} / \mathrm{min}$ hydrogen flow rate in the presence of $2 \mathrm{~mL}$ nanopure water without using dichloromethane organic solvent. 


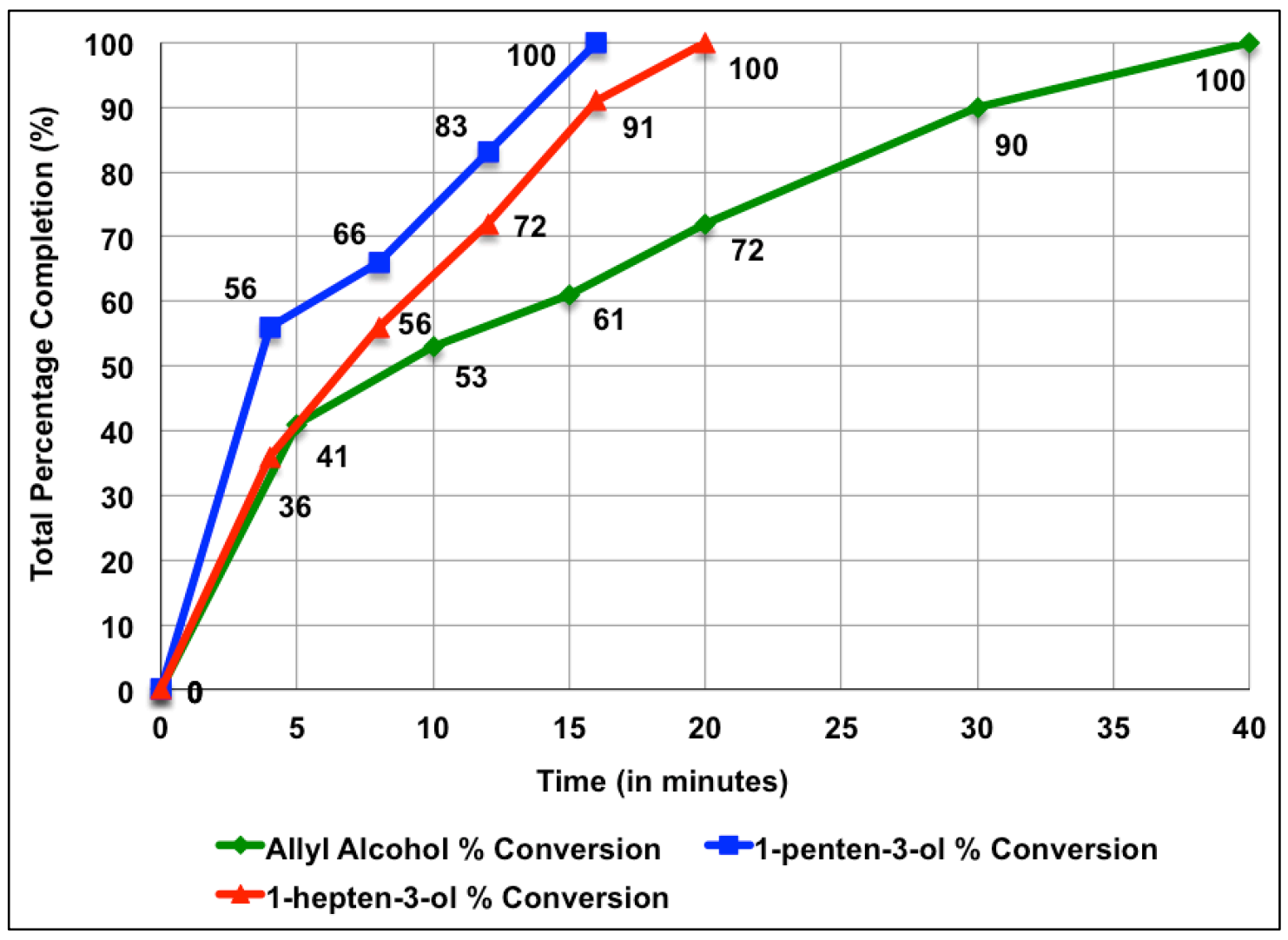

Figure 5.9. Plot showing the total percentage completion of the catalytic reaction vs. time (in minutes) for biphasic solvent mixture containing $2 \mathrm{~mL}$ of as-synthesized aqueous Pd-citrate NPs solution and $2 \mathrm{~mL}$ dichloromethane and $19.9 \pm 0.7 \mathrm{~mL} / \mathrm{min}$ hydrogen flow rate in the presence of $0.74 \times 10^{-3}$ moles of (a) allyl alcohol, (b) 1-penten-3-ol and (c) 1-hepten-3-ol. 
$19.9 \pm 0.7 \mathrm{~mL} / \mathrm{min}$ of hydrogen flow rate in biphasic $2 \mathrm{~mL}$ dichloromethane and $2 \mathrm{~mL}$ aqueous solvent mixture. The reaction showed 38\% product conversion in 60 minutes after which the reaction didn't go further as the solution became unstable and clear as shown in Figure 5.10. Clearly having Pd NPs as the catalyst is better than a Pd (II) salt for this particular salt.

\subsubsection{Electronic Effects of CuPd core-shell citrate-stabilized NPs for Improving Reaction Selectivity}

One of our goals was to improve the selectivity of the hydrogenation/ isomerization reaction of allylic alcohols, which we discovered could be achieved by modifying the metal core in a core-shell metal/Pd structure. These new metal NPs have different electronic properties and thus may change the selectivity for the reaction while keeping the same ligands. In order to test this possibility, we synthesized CuPd core-shell NPs capped with citrate ligands and measured the catalytic activity for the hydrogenation/isomerization of 1-penten-3-ol substrate. The CuPd-citrate NPs were synthesized under ice-cold temperature by first adding $50 \mu \mathrm{L}$ of $0.1 \mathrm{M}$ each of $\mathrm{CuSO}_{4} \cdot 5 \mathrm{H}_{2} \mathrm{O}$ and sodium citrate in $20 \mathrm{~mL}$ of nanopure water under constant nitrogen purging. Then, $1 \mathrm{~mL}$ of freshly prepared $\mathrm{NaBH}_{4}(4 \mathrm{mg}$ in 4 $\mathrm{mL}$ nanopure water) was added to the reaction mixture rapidly. The color of the reaction mixture turned brown which was stirred for 15 minutes. Lastly, $100 \mu \mathrm{L}$ of $\mathrm{K}_{2} \mathrm{PdCl}_{4}(0.1 \mathrm{M})$ was added and reaction mixture was stirred for another 20 minutes. The CuPd-citrate NPs were stored in a vial at room temperature for at least 24 hours before use. These CuPd-citrate NPs were shown to be quite stable in $2 \mathrm{~mL}$ 

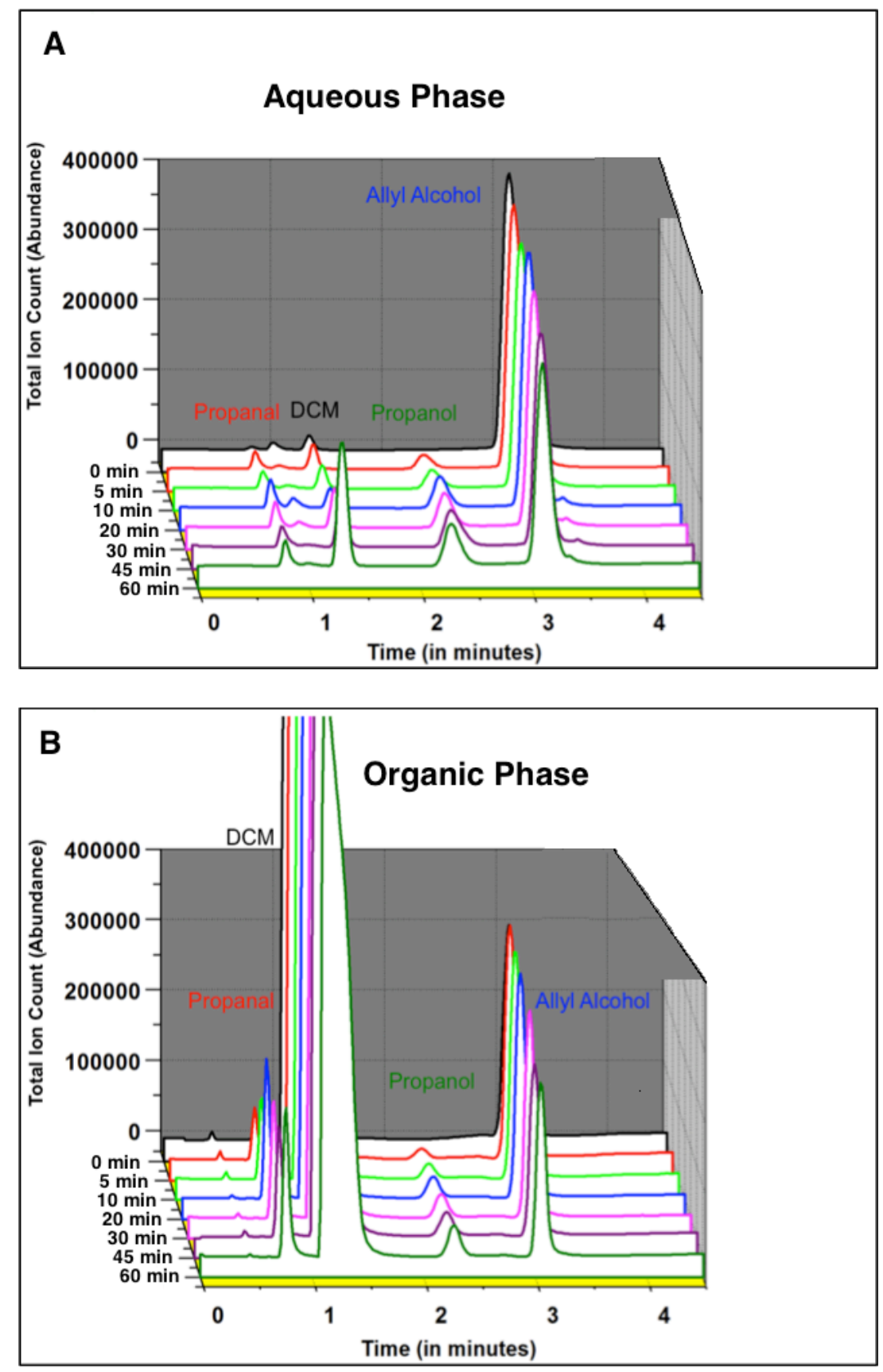

Figure 5.10. GC-FID spectra of (A) aqueous and (B) organic phases for the catalytic activity of $0.15 \mathrm{mg}$ of $\mathrm{PdCl}_{4}^{2-}$ salt in aqueous solution for $0.74 \times 10^{-3}$ moles of allyl alcohol at $19.9 \pm 0.7 \mathrm{~mL} / \mathrm{min}$ of hydrogen flow rate in biphasic 2 $\mathrm{mL}$ dichloromethane and $2 \mathrm{~mL}$ aqueous solvent mixture. 
dichloromethane-2 $\mathrm{mL}$ aqueous biphasic solvent system even after an hour, which was used for the catalytic reaction under hydrogen gas purging at a flow rate of $19.9 \pm 0.7 \mathrm{~mL} / \mathrm{min}$ at room temperature and atmospheric pressure. $20 \mu \mathrm{L}$ aliquots were removed from both aqueous and organic phases at particular intervals of time. The progress of the catalysis reaction was followed by gas chromatography (GCFID) of samples before exposure to hydrogen and after addition of catalyst and exposure to hydrogen at different intervals of time. Figure 5.11. shows the GC spectra of the organic dichloromethane layer (Frame A) and of the aqueous (Frame B) at various times for the biphasic catalysis of $0.74 \times 10^{-3}$ moles of 1-penten-3-ol dissolved in $2 \mathrm{~mL}$ of as-synthesized aqueous CuPd-citrate NPs in the presence of $19.9 \pm 0.7 \mathrm{~mL} / \mathrm{min}$ hydrogen flow rate for $0,5,10,20,30,45$ and 60 minutes. In the aqueous phase, the peak corresponding to 1-penten-3-ol coming at 3.65 minutes decreased with time as expected for it reacting with hydrogen, whereas the peaks corresponding to 3-pentanone and 3-pentanol at times 1.97 and 3.15 minutes, respectively, increased. Also, a stable intermediate peak at 2.45 minute showed up, which eventually vanished after the completion of the reaction. In the organic phase, the peak heights for both products increased largely with time as the 1-penten-3-ol peak height decreased and eventually vanished completely, showing the presence of both products at 60 minutes. The organic layer was separated from the aqueous layer and product trapped inside the aqueous layer was further extracted by washing with $2 \mathrm{~mL}$ of dichloromethane twice to remove the remaining products. The catalytic reaction showed a higher selectivity for 3-pentanol (hydrogenated product) than 3-pentanone (isomerized product) in the ratio of 95:5 due to the 

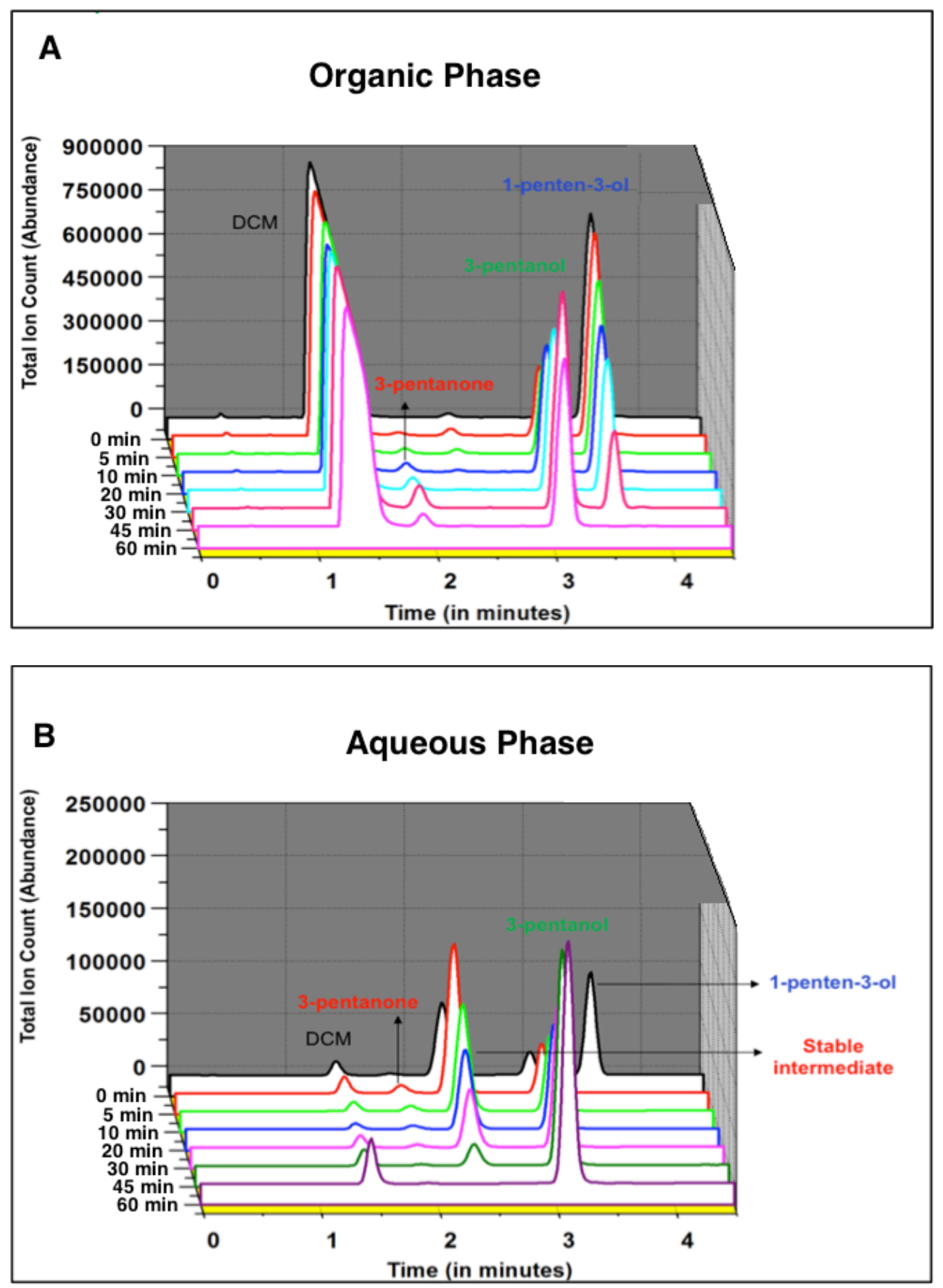

Figure 5.11. GC-FID spectra of (A) organic and (B) aqueous phases for the catalytic activity in biphasic solvent mixture containing $2 \mathrm{~mL}$ of assynthesized aqueous citrate-capped CuPd NPs solution and $2 \mathrm{~mL}$ dichloromethane with $0.74 \times 10^{-3}$ moles of 1 -penten-3-ol at $19.9 \pm 0.7 \mathrm{~mL} / \mathrm{min}$ of hydrogen flow rate for 60 minutes. 


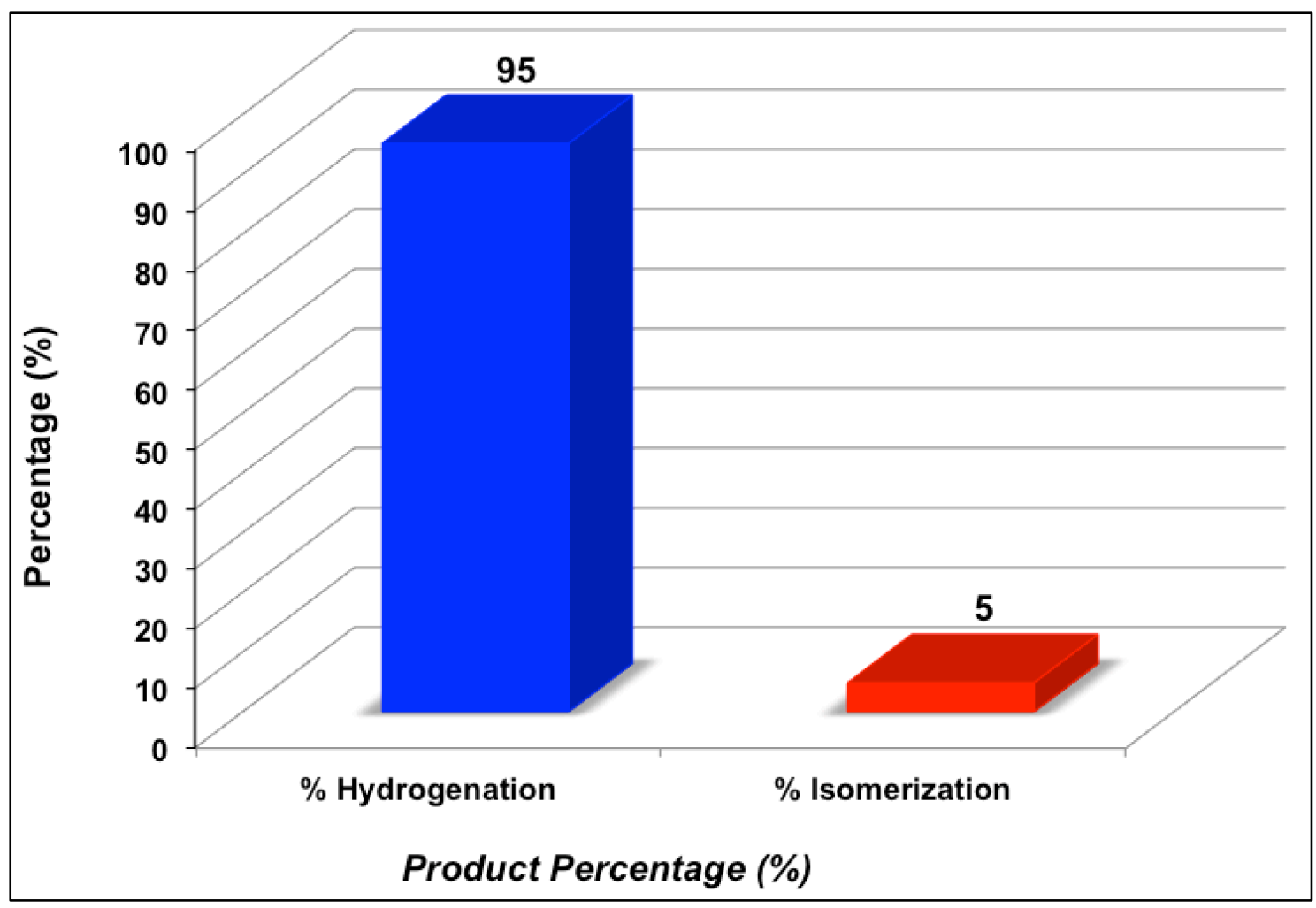

Figure 5.12. Plot showing the selectivity of the citrate-capped CuPd NPs for the catalytic reaction of $0.74 \times 10^{-3}$ moles of 1 -penten-3-ol at $19.9 \pm 0.7$ $\mathrm{mL} / \mathrm{min}$ of hydrogen flow rate for 60 minutes. 
electronic effect caused by the presence of $\mathrm{Cu}$ in Pd core. The total TOF for the hydrogenation/ isomerization reaction was 773 moles product/moles $\mathrm{Pd} /$ hour as CuPd-citrate NPs became unstable during the reaction. The TOF was 737 for hydrogenation and 36 for isomerization. Figure 5.12 . shows a plot of the selectivity of the citrate-capped $\mathrm{Cu} / \mathrm{Pd} \mathrm{NPs}$ for the catalytic reaction of 1-penten-3-ol.

\subsection{CONCLUSIONS}

In summary, we reported the successful synthesis of water-soluble Pd NPs coated with citrate ligands that shows high stability under biphasic solvent system and high catalytic performances for hydrogenation/isomerization reactions with better turnover frequencies (TOFs) and recyclability with 100\% product conversion based on the substrate structure for the first time. Pd-citrate NPs showed higher stability under dichloromethane organic solvent than ethyl acetate and chlorobenzene solvents, showing its suitability for the hydrogenation/isomerization reactions. Pd NPs also showed high stability under a constant flow of hydrogen gas for at least 1 hour according to UV/Vis-spectroscopy, which was necessary for the catalytic reaction. We observed that the substrate structure plays an important role in the catalytic activity of the NPs resulting in 1-penten-3-ol being catalytically more active than allyl alcohol and 1-hepten-3-ol due to its appropriate hydrocarbon chain length that facilitates the orientation of substrate active site towards the catalyst present in the aqueous phase. The catalytic activity of Pd-citrate NPs shows less TOF

and low recyclability for 2-methyl-3-buten-2-ol when compared with 1-penten-3-ol with same number of carbon atoms. This confirms that straight chain substrates are 
catalytically more active that branched substrate with the same number of carbon atoms. Also, use of biphasic solvent system was observed to be more efficient than using of a mono-phasic aqueous solvent system for the hydrogenation/ isomerization reaction of unsaturated alcohols showing high NPs stability, high TOF, and high recyclability with $100 \%$ product conversion. The selectivity chart for Pdcitrate NPs towards hydrogenated and isomerized products after 1 complete cycle of reaction for all substrates was about 3:2. We also synthesized citrate-capped CuPd core-shell NPs and used them for the hydrogenation/isomerization of 1penten-3-ol. Interestingly, CuPd-citrate NPs showed a high selectivity with $\sim 95 \%$ preference for hydrogenated product, although with much lower TOF compared to Pd-citrate NPs. These studies will help us to better understand the catalytic behavior of citrate-coated Pd NPs for various substrates and their utilization in designing better catalysts with high stability, catalytic activity, selectivity, and recyclability. Thus, due to their high accessibility, well-defined surface morphology and high stability in the biphasic solution, Pd-citrate NPs show great promise as convenient and active pseudo-homogeneous catalysts. 


\section{CHAPTER VI}

\section{CATALYSIS STUDIES OF ALKYLAMINE-STABILIZED PD NANOPARTICLES}

(NPs) FOR SUZUKI-MIYAURA REACTION

\subsection{INTRODUCTION}

The Suzuki cross-coupling reactions of arylboronic acids and aryl halides provide an effective synthetic route to form biaryls ${ }^{162-164}$ as shown in Figure 6.1. The coupling reaction of arylboron derivatives with aryl halides in the presence of $\mathrm{Pd}\left(\mathrm{PPh}_{3}\right)_{4}$ and base to afford biaryls was first reported in $1979 .{ }^{165}$ Since then, a number of modifications have appeared. Suzuki coupling reactions present wide applications in the production of polymers, poly-olefins, styrene, substituted biphenyls, agrochemicals, pharmaceutical intermediates, and high-tech materials. ${ }^{166}$, 167 It is a key coupling reaction in the synthesis of a variety of marketed products including the blood pressure medications valsartan and losartan, the antifungal agrochemical boscalid, and even OLEDs (Organic Light-Emitting Diodes) used in TVs and monitors. ${ }^{166}$ This widespread use of Suzuki cross-coupling reactions is mainly due to the mild conditions associated with these reactions together with their tolerance to a wide variety of functional groups and availability of diverse boronic acids and the easy handling and removal of boron-containing byproducts 


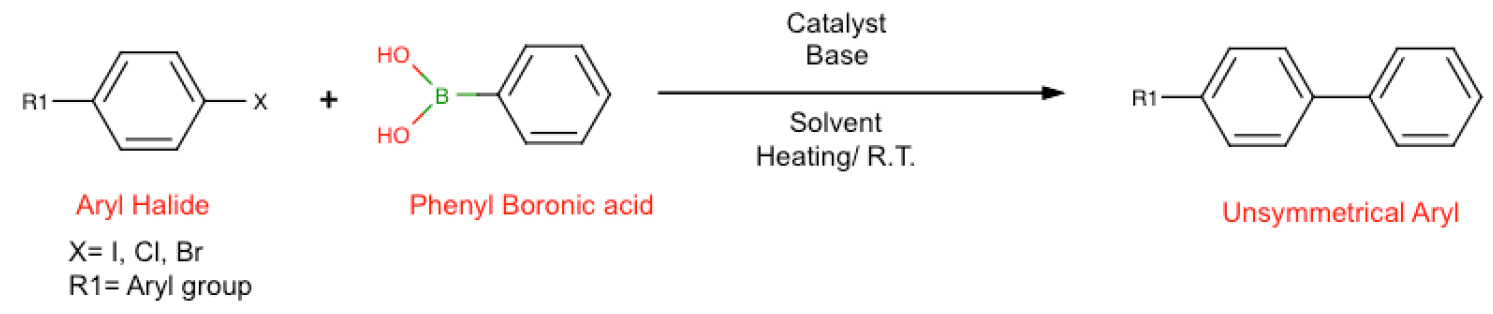

Figure 6.1. Reaction scheme showing the utilization of aryl halide and phenylboronic acid in the presence of a catalyst, base and solvent under heating to form unsymmetrical biaryl product for C-C cross coupling SuzukiMiyaura reaction. 
when compared to other organometallic reagents. These reactions are carried out in organic solvents and catalyzed by various Pd/ligand systems. ${ }^{168}$ Colloidal metal particles are effective catalysts for the chemical transformations due to their large surface area. ${ }^{169}$ It has been shown that palladium colloids on the nanometer length scale are effective catalysts for the Suzuki reactions ${ }^{170}$ in organic solvents due to their milder reaction conditions as well as more environmentally friendly methods. Nevertheless, one serious problem in homogeneous metal catalysis is separation of the reaction products from the catalysts.

\subsection{RESULTS AND DISCUSSION}

The Pd catalyzed C-C cross coupling of alkyl halides and aryl boronic acids, known as Suzuki-Miyaura reaction, has been widely used since the last three decades for synthesizing unsymmetrical biaryls. Since the Suzuki reaction involves heating of the reaction mixture, there was a need to synthesize Pd NPs, which can tolerate the high temperature used in the Suzuki reaction, yet reactive enough to get $100 \%$ product yield. Previously, in our lab, Zamborini and co-workers have synthesized Pd NPs coated with $\mathrm{C}_{2} \mathrm{NH}_{2}, \mathrm{C}_{12} \mathrm{NH}_{2}$ and $\mathrm{C} 16 \mathrm{NH}_{2}$ ligands and showed that $\mathrm{C}_{16 \mathrm{NH}_{2}} \mathrm{Pd} \mathrm{NPs}$ are stable under hydrogen conditions due to the presence of protective bilayer formation of ligands on $\mathrm{Pd}$ core and still reactive enough to provide high TOFs. ${ }^{75}$ Thus, we used the toluene solutions of same Pd NPs coated with $\mathrm{C}_{8 \mathrm{NH}_{2}}, \mathrm{C} 12 \mathrm{NH}_{2}$ and $\mathrm{C} 16 \mathrm{NH}_{2}$ ligands and determined their thermal stability to be used as potential catalysts. We used UV-Vis absorbance spectrum as a function of temperature ranging from 25 to $95^{\circ} \mathrm{C}$ to determine the stability. The UV-visible 
spectra show that the absorbance for $\mathrm{C}_{16 \mathrm{NH}_{2}}$ Pd NPs remains high for all temperatures due to the protective bilayer coating of $\mathrm{C}_{16} \mathrm{NH}_{2}$ ligands on the $\mathrm{Pd}$ core, ${ }^{75}$ whereas the absorbance for $\mathrm{C}^{12} \mathrm{NH}_{2} \mathrm{Pd}$ and $\mathrm{C} \mathrm{NH}_{2}$ Pd NPs decreased rapidly at high temperatures which shows temperature-induced aggregation. The lower stability of these NPs is due to the weak interaction of amines with the Pd core and the shorter chain-length. Thus, we decided to use and study $\mathrm{C}_{16 \mathrm{NH}_{2}} \mathrm{Pd} \mathrm{NPs}$ as catalyst for the Suzuki reaction. The $\mathrm{C}_{16 \mathrm{NH}_{2}} \mathrm{Pd} \mathrm{NPs}$ were again tested in toluene solution using UV-Vis spectroscopy as a function of different temperatures ranging from 30 to $110^{\circ} \mathrm{C}$ each heated for at least 30 minutes. The absorbance remained high for all temperature, which shows good overall thermal stability suitable for use as a catalyst in the Suzuki reaction.

\subsubsection{Catalytic Activity of alkylamine-capped Pd NPs}

In this study, we describe the catalytic activity of $\mathrm{C} 16 \mathrm{NH}_{2}$ Pd NPs synthesized under biphasic conditions to study the Suzuki-Miyaura reaction between 4iodoanisole and phenylboronic acid used as a model reaction, since 4-iodoanisole is solid and non-volatile. Hence, it can be easily detected on a TLC plate and with GCMS. Also, the $-\mathrm{OCH}_{3}$ group is an activating group in electrophilic-aromatic substitution reactions, which helps to precede the reaction further, and thus can be used as a starting material. Also, we can see any homo-coupled biphenyl product if formed as it will be different from the 4-methoxyphenyl benzene product.

The procedure for the Suzuki-Miyaura reaction involved mixing various mole ratios of arylhalide (4-iodoanisole), arylboronic acid (phenylboronic acid) and 
$\mathrm{C}_{16} \mathrm{NH}_{2} \mathrm{Pd} \mathrm{NPs}$ as the catalyst in various solvent systems, where the base is dissolved in a minimal amount of water and the reaction mixture is heated at a particular temperature as shown in Table 6.1. The progress of the catalytic reaction was followed by thin-layer chromatography (TLC) and gas chromatography-mass spectrometry (GC-MS) for samples before and after addition of NP catalyst for different times. We performed various reactions involving different reagents in various mole ratios and finally we were able to achieve the right reaction conditions.

We mixed 4-iodoanisole (1.0 mole equi.), phenylboronic acid (1.3 mole equi.), and Pd NPs catalyst (1.5 mole \%) in toluene:water (12 mL:1 mL) biphasic mixture, where potassium carbonate ( 4.0 mole equi.) as base was dissolved in water, and heated the reaction mixture at $70^{\circ} \mathrm{C}$ as shown in Figure 6.2. The reaction was complete with the entire limiting reagent being consumed within 4 hours. After completion of the reaction, the product formed was confirmed by TLC and GC-MS as shown in Figure $6.3(\mathrm{~A})$ and $(\mathrm{C})$. Both techniques showed the completion of the Suzuki reaction with the formation of product and a little side product. GC-MS determined the side product was biphenyl formed by the homo coupling of phenylboronic acid. Unfortunately, the $\mathrm{C}_{16 \mathrm{NH}_{2}} \mathrm{Pd} \mathrm{NPs}$ used for this reaction as catalyst got aggregated after the completion of the reaction as shown in Figure 6.3. (B). The gas chromatogram of the reaction involving 4-iodoanisole and phenylboronic acid showed fusion of excess phenylboronic acid to form triphenylboroxane and formation of 4-methoxyphenyl benzene as product at their respective retention times. 


\begin{tabular}{|c|c|c|c|c|c|c|c|c|c|}
\hline Reactions & 1 & 2 & 3 & 4 & 5 & 6 & 7 & 8 & 9 \\
\hline $\begin{array}{l}\text { 4-lodo } \\
\text { anisole }\end{array}$ & $\begin{array}{l}0.500 \mathrm{~g} \\
(1.0 \mathrm{eq})\end{array}$ & $\begin{array}{l}0.500 \mathrm{~g} \\
(1.0 \mathrm{eq})\end{array}$ & $\begin{array}{l}0.500 \mathrm{~g} \\
(1.0 \mathrm{eq})\end{array}$ & $\begin{array}{l}0.500 \mathrm{~g} \\
(1.0 \mathrm{eq})\end{array}$ & $\begin{array}{l}0.500 \mathrm{~g} \\
(1.0 \mathrm{eq})\end{array}$ & $\begin{array}{l}0.500 \mathrm{~g} \\
(1.0 \mathrm{eq})\end{array}$ & $\begin{array}{l}0.500 \mathrm{~g} \\
(1.0 \mathrm{eq})\end{array}$ & $\begin{array}{l}0.500 \mathrm{~g} \\
(1.0 \mathrm{eq})\end{array}$ & $\begin{array}{l}0.500 \mathrm{~g} \\
(1.0 \mathrm{eq})\end{array}$ \\
\hline $\begin{array}{l}\text { Phenyl } \\
\text { boronic acid }\end{array}$ & $\begin{array}{l}0.448 \mathrm{~g} \\
(1.5 \mathrm{eq})\end{array}$ & $\begin{array}{l}0.448 \mathrm{~g} \\
(1.5 \mathrm{eq})\end{array}$ & $\begin{array}{l}0.448 \mathrm{~g} \\
(1.5 \mathrm{eq})\end{array}$ & $\begin{array}{l}0.448 \mathrm{~g} \\
(1.5 \mathrm{eq})\end{array}$ & $\begin{array}{l}0.388 \mathrm{~g} \\
(1.3 \mathrm{eq})\end{array}$ & $\begin{array}{l}0.388 \mathrm{~g} \\
(1.3 \mathrm{eq})\end{array}$ & $\begin{array}{l}0.388 \mathrm{~g} \\
(1.3 \mathrm{eq})\end{array}$ & $\begin{array}{l}0.388 \mathrm{~g} \\
(1.3 \mathrm{eq})\end{array}$ & $\begin{array}{l}0.388 \mathrm{~g} \\
(1.3 \mathrm{eq})\end{array}$ \\
\hline $\begin{array}{c}12 \times C 16- \\
\mathrm{C} 18 \mathrm{NH} 2 \mathrm{Pd}\end{array}$ & $\begin{array}{l}10 \mathrm{mg} \mathrm{(1} \\
\mathrm{mole} \%)\end{array}$ & $\begin{array}{l}10 \mathrm{mg} \mathrm{(1} \\
\mathrm{mole} \%)\end{array}$ & $\begin{array}{l}10 \mathrm{mg} \mathrm{(1} \\
\text { mole\%) }\end{array}$ & $\begin{array}{l}20 \mathrm{mg} \mathrm{(2} \\
\text { mole\%) }\end{array}$ & $\begin{array}{l}10 \mathrm{mg} \mathrm{(1} \\
\mathrm{mole} \%)\end{array}$ & $\begin{array}{l}10 \mathrm{mg} \mathrm{(1} \\
\mathrm{mole} \%)\end{array}$ & $\begin{array}{c}15 \mathrm{mg} \\
(1.5 \mathrm{~mole} \\
\%)\end{array}$ & $\begin{array}{c}15 \mathrm{mg} \\
(1.5 \mathrm{~mole} \\
\%)\end{array}$ & $\begin{array}{l}20 \mathrm{mg} \mathrm{(2} \\
\text { mole } \%)\end{array}$ \\
\hline Base & $\begin{array}{l}\mathrm{NaOH} \\
0.294 \mathrm{~g} \\
(3.0 \text { eq) }\end{array}$ & $\begin{array}{l}\mathrm{NaOH} \\
0.294 \mathrm{~g} \\
(3.0 \text { eq) }\end{array}$ & $\begin{array}{l}\mathrm{NaOH} \\
0.294 \mathrm{~g} \\
(3.0 \mathrm{eq})\end{array}$ & $\begin{array}{l}\mathrm{NaOH} \\
0.294 \mathrm{~g} \\
(3.0 \text { eq) }\end{array}$ & $\begin{array}{c}\text { Triethyl } \\
\text { amine } \\
1.368 \mathrm{~mL} \\
(4.0 \mathrm{eq})\end{array}$ & $\begin{array}{c}\text { Triethyl } \\
\text { amine } \\
1.368 \mathrm{~mL} \\
\text { (4.0eq) }\end{array}$ & $\begin{array}{c}\mathrm{KF} \\
0.570 \mathrm{~g} \\
(4.0 \mathrm{eq})\end{array}$ & $\begin{array}{l}\mathrm{K}_{2} \mathrm{CO}_{3} \\
1.355 \mathrm{~g} \\
(4.0 \text { eq) }\end{array}$ & $\begin{array}{l}\mathrm{K}_{2} \mathrm{CO}_{3} \\
1.355 \mathrm{~g} \\
(4.0 \text { eq) }\end{array}$ \\
\hline Solvent 1 & $\begin{array}{c}\text { THF } \\
(16 \mathrm{~mL})\end{array}$ & $\begin{array}{c}\text { THF } \\
(16 \mathrm{~mL})\end{array}$ & $\begin{array}{c}\text { DCM } \\
(16 \mathrm{~mL})\end{array}$ & $\begin{array}{c}\text { THF } \\
(16 \mathrm{~mL})\end{array}$ & $\begin{array}{c}\text { THF } \\
(16 \mathrm{~mL})\end{array}$ & $\begin{array}{c}\text { THF } \\
(16 \mathrm{~mL})\end{array}$ & $\begin{array}{c}\text { THF } \\
(16 \mathrm{~mL})\end{array}$ & $\begin{array}{l}\text { Dioxan } \\
(16 \mathrm{~mL})\end{array}$ & $\begin{array}{c}\text { THF } \\
(16 \mathrm{~mL})\end{array}$ \\
\hline Solvent 2 & $\begin{array}{l}\text { Water } \\
(8 \mathrm{~mL})\end{array}$ & $\begin{array}{l}\text { Water } \\
(8 \mathrm{~mL})\end{array}$ & $\begin{array}{l}\text { Water } \\
(8 \mathrm{~mL})\end{array}$ & $\begin{array}{l}\text { Water } \\
(8 \mathrm{~mL})\end{array}$ & - & - & $\begin{array}{l}\text { Water } \\
(8 \mathrm{~mL})\end{array}$ & $\begin{array}{l}\text { Water } \\
(8 \mathrm{~mL})\end{array}$ & $\begin{array}{l}\text { Water } \\
(8 \mathrm{~mL})\end{array}$ \\
\hline $\begin{array}{l}\text { Heating } \\
\left(\text { at } 70^{\circ} \mathrm{C}\right)\end{array}$ & $\begin{array}{l}\boldsymbol{V} \text { (yes) } \\
\text { For } 8 \\
\text { hrs }\end{array}$ & $\begin{array}{l}\mathbf{x} \text { (no) } \\
\text { R.T. for } \\
24 \text { hrs }\end{array}$ & $\begin{array}{c}\boldsymbol{x} \\
\text { For } 24 \\
\text { hrs }\end{array}$ & $\begin{array}{l}\text { For } 12 \\
\text { hrs }\end{array}$ & $\begin{array}{l}\text { For } 8 \\
\text { hrs }\end{array}$ & $\begin{array}{c}\boldsymbol{x} \\
\text { For } 24 \\
\text { hrs }\end{array}$ & $\begin{array}{c}\boldsymbol{x} \\
\text { For } 24 \\
\text { hrs }\end{array}$ & $\begin{array}{c}\boldsymbol{x} \\
\text { Sonicate } \\
\text { for } 2 \text { hrs }\end{array}$ & $\begin{array}{l}\text { For } 4 \\
\text { hrs }\end{array}$ \\
\hline $\begin{array}{l}\text { Product } \\
\text { Obtained }\end{array}$ & $\boldsymbol{x}$ & $\boldsymbol{x}$ & $\boldsymbol{x}$ & $\boldsymbol{x}$ & $\boldsymbol{x}$ & $\boldsymbol{x}$ & $x$ & $\sim \checkmark$ & $\checkmark$ \\
\hline
\end{tabular}

Table 6.1. Table of the different reaction conditions involving the mixing of 4iodoanisole, phenylboronic acid and Pd NP catalyst in organic:aqueous biphasic mixture, where base is dissolved in water and the reaction is either heated or stirred at room temperature for different amounts of time. The reaction condition 9 showed the best results. 


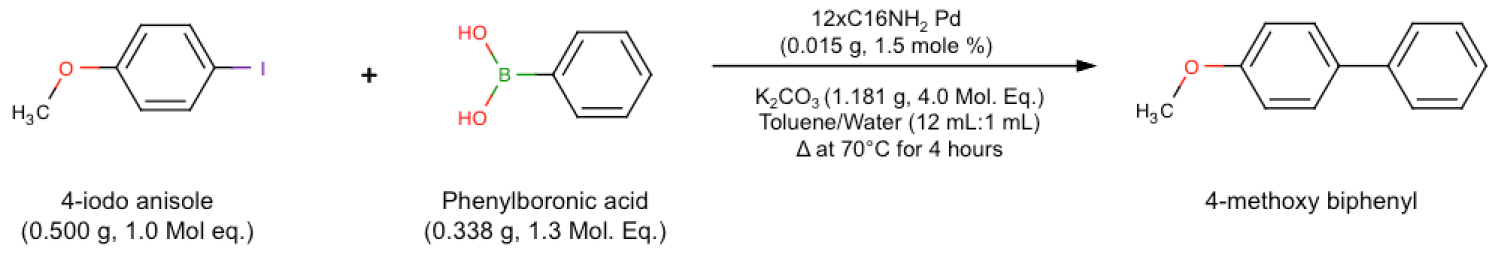

Figure 6.2. Reaction scheme showing the best possible reaction conditions involving the mixing of 4-iodoanisole (1.0 mole equivalent), phenylboronic acid (1.3 mole equivalent) and Pd NPs catalyst (1.5 mole \%) in toluene:water (12 mL:1 mL) biphasic mixture, where potassium carbonate (4.0 mole equivalent) base was dissolved in water, and the reaction mixture heated at $70^{\circ} \mathrm{C}$. 

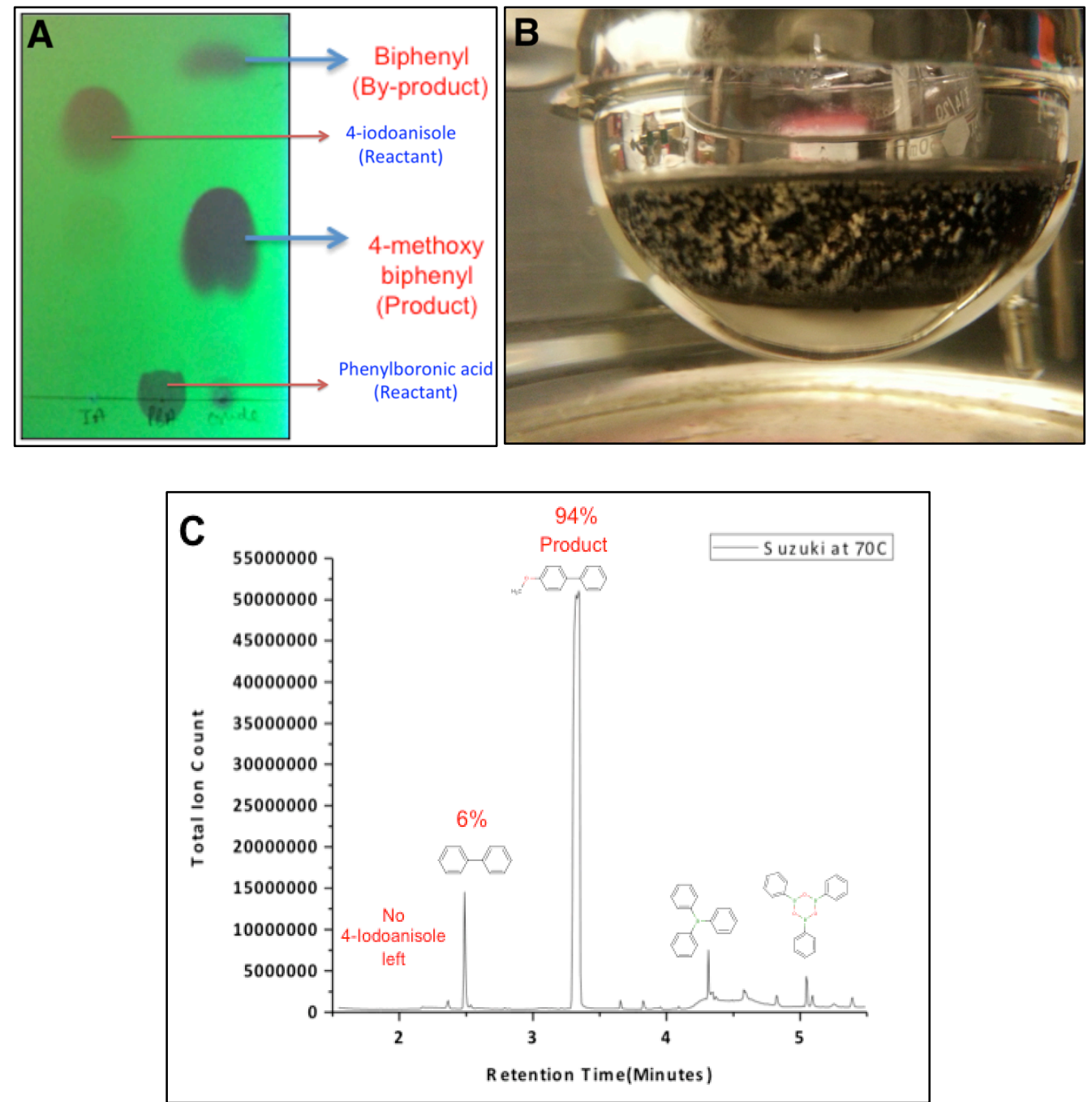

Figure 6.3. Product confirmation for the model Suzuki-Miyaura reaction involving 4-iodoanisole (0.500 gms, 1.0 mole equi.), phenylboronic acid (0.338 gms, 1.3 mole equi.), $\mathrm{C}_{16 \mathrm{NH}_{2}} \mathrm{Pd} \mathrm{NPs}$ (0.015 gms, 1.5 mole \%), $\mathrm{K}_{2} \mathrm{CO}_{3}$ as base (1.181 gms, 4.0 mole equi.) and toluene:water (12 mL:1 mL) biphasic solvent mixture at $70^{\circ} \mathrm{C}$ showing (A) TLC and (B) Aggregation of $\mathrm{C}^{16 \mathrm{NH}_{2} \mathrm{Pd}}$ NPs after completion of the reaction and (C) Gas chromatography of the final reaction mixture. Products were confirmed using mass spectroscopy. 
We also performed the experiment by adding primary reactants (4iodoanisole and pheylboronic acid) as solution in organic solvent drop wise in the reaction solution to get the products heating at $70^{\circ} \mathrm{C}$ while keeping the catalyst system stabilized. We used various ratios of catalyst:reactant mixture ranging from $1 / 10,000,1 / 1000,1 / 100$ and $1 / 10$ and measured the UV-Vis spectra of the catalyst dissolved in the reaction mixture for different amounts of time ranging from 0,15 , 30, 45 and 60 minutes as shown in Figure 6.4. The $\mathrm{C}_{16 \mathrm{NH}_{2}}$ Pd NPs used for this reaction as catalyst showed high stability at mole ratios of $1 / 10,000,1 / 1000$ and $1 / 100$ as seen by the high absorbance with decrease in the wavelength with no plasmon band. For ratio $1 / 10$, the UV-Vis spectra for $\mathrm{C}^{16 \mathrm{NH}_{2}}$ Pd NPs showed a peak at $\sim 250 \mathrm{~nm}$ which is due to the formation of $\mathrm{Pd}(\mathrm{II})$ species. Also, the $\mathrm{C}_{16 \mathrm{NH}_{2}} \mathrm{Pd} \mathrm{NPs}$ showed a decrease in the absorbance with decrease in wavelength, showing low stability. This shows that the presence of substrate has an effect on the Pd core surface where the reaction occurs that leads to the oxidation of Pd atoms to Pd(II) species and little NP aggregation.

More work is needed to solve this problem of aggregation by adding solution of both primary reactants in toluene drop wise so as to stop the aggregation of NPs, but still this study has led to interesting insights regarding the effects of various reaction parameters and choice of alkylamine as a ligand for Suzuki-Miyaura reactions. 


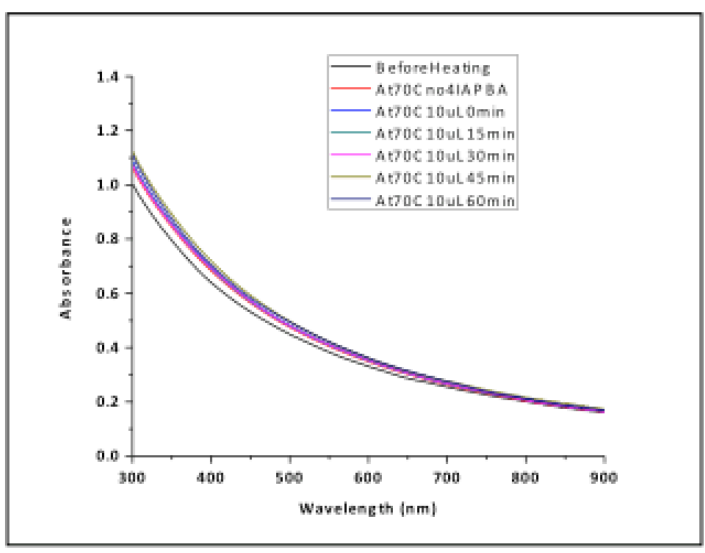

$1 / 10,000$

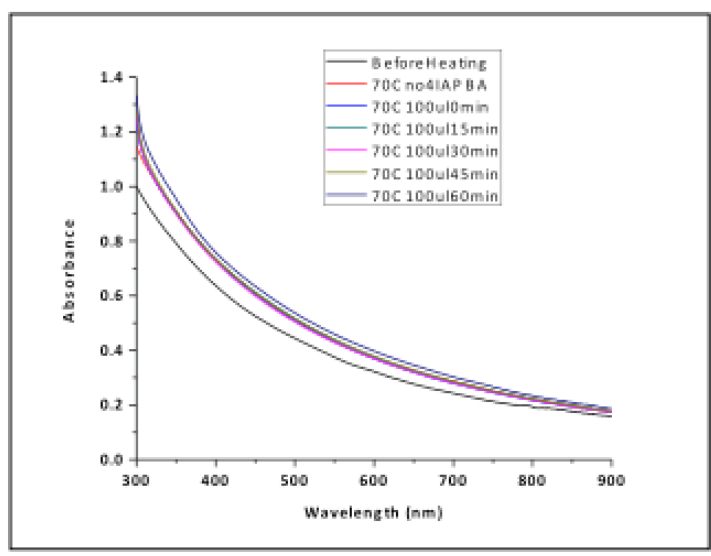

$1 / 100$

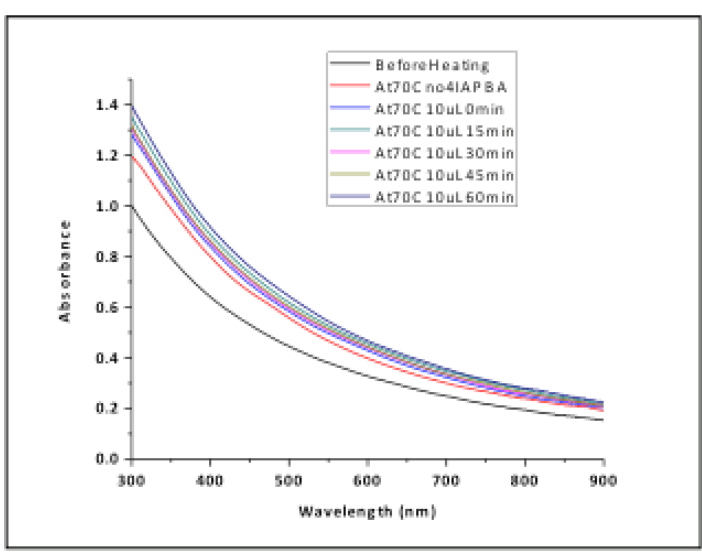

$1 / 1000$

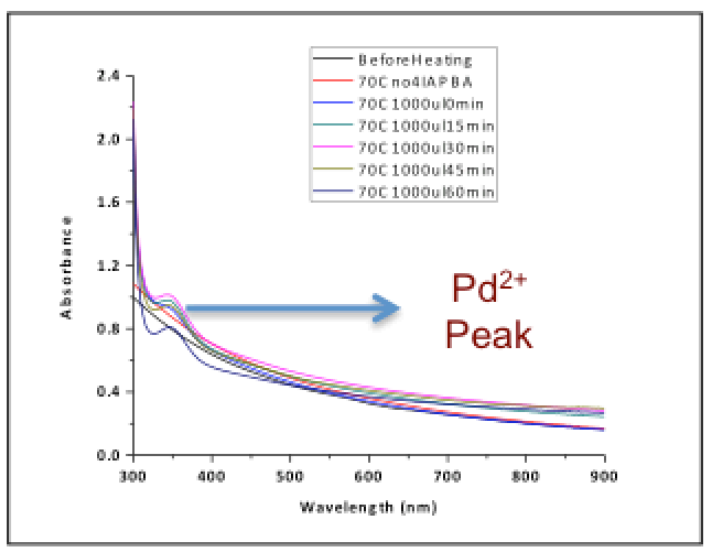

$1 / 10$

Figure 6.4. UV-vis spectra of the catalyst dissolved in the reaction mixture containing various ratios of catalyst: reactant mixture ranging from $1 / 10,000,1 / 1000,1 / 100$ and $1 / 10$ for different amounts of time ranging from $0,15,30,45$ and 60 minutes. 


\subsubsection{Comparison with Other Nanoparticle Catalysts}

Reetz and co-workers were the first to report the use of $\mathrm{Pd}$ and $\mathrm{Pd} / \mathrm{Ni}$ nanoparticles for the Suzuki coupling of aryl bromides and chlorides with phenylboronic acid using $2 \mathrm{~mol} \%$ of these catalysts. ${ }^{170}$ El-Sayed and co-workers initially reported the use of Pd NPs stabilized by PVP as catalysts in the coupling of aryl iodides with phenylboronic acid in aqueous media. ${ }^{171}$ The rate of the reaction was shown to depend on the catalyst concentration, indicating that the Suzuki reaction occurred at the metallic surface. El-Sayed and co-workers also reported the use of Pd NPs stabilized by poly(amido-amine) dendrimers of different generations (D2-D4) bearing hydroxyl groups and compared the stability of the resulting nanoparticles by measuring the amount of Pd black formed during the reaction..$^{3,43 \text {, }}$ 172, 173 The nanoparticles stabilized by a mixture of polymer D3 and PVP were the most efficient catalysts. The same group directly compared the catalytic activity and stability of polyvinylpyrrolidone (PVP)-stabilized Pd NPs in solution to PVP-Pd NPs adsorbed onto carbon for catalyzing the Suzuki coupling reaction. They observed that carbon-supported Pd NPs, although less catalytically active, were highly recyclable making them potentially better catalyst for the Suzuki reaction compared to colloidal NPs. ${ }^{3}$

Astruc and coworkers have reported to synthesize dodecanthiolatestabilized PdNPs capable of catalyzing Suzuki-Miyaura coupling reaction ${ }^{36}$. From this work, they were able to successfully demonstrate for the first time that straightchain alkanethiolate capped PdNPs could be used to catalyze a simple reaction under mild reaction conditions. These catalysts showed modest yields, but they 
were phosphine-free and recyclable 6 times with yield lowered down to 87\%. Later, Fornasiero and coworkers reported an easy approach for the preparation of functionalized Pd NPs by directly employing 11-mercaptoundecanoic acid (MUA) and mixed ligand monolayers of 9-mercapto-1-nonaol and 1-dodecanethiol as stabilizers and their easy recycling and reusability as heterogeneous catalyst in Suzuki cross coupling reaction. ${ }^{79}$ They showed recyclability of up to 5 times with iodobenzene or 4-bromobenzonitrile with a little decrease in the activity. The Kaifer group ${ }^{175}$ and Newmann group ${ }^{176}$ reported the catalytic activity for the SuzukiMiyaura reactions of thiolate-Pd NPs modified, respectively, with $\beta$-cyclodextrin and polyoxometallates. Fox and co-workers reported the catalytic behavior, including for the Suzuki-Miyaura reaction, of Pd NP-cored dendrimers. ${ }^{174}$ The preliminary investigations had shown Pd G-3 NPs as an efficient catalyst for the Suzuki and Heck reactions. An important challenge for this reaction is to obtain $100 \%$ yield and recycle the catalyst used in the reaction at the same time for number of cycles. Also, we were curious to know the reactivity of alkylamine-coated Pd NPs, as they are stable under hydrogen as seen before due to the bilayer structure and they haven't been utilized for C-C cross coupling Suzuki-Miyaura reaction before, according to

our knowledge, as per the literature. Therefore, our goal was to better understand the catalytic properties of alkylamine-coated Pd NPs and improve its reactivity and recyclability for the Suzuki reaction.

\subsection{CONCLUSIONS}

In summary, we reported the successful synthesis of alkylamine-coated Pd 
NPs coated with $\mathrm{C}_{16 \mathrm{NH}_{2}}$ ligands that shows high stability under thermal-induced aggregation and good catalytic performance for C-C cross coupling of 4-iodo anisole and phenylboronic acid as Suzuki-Miyaura reaction with $100 \%$ product conversion for the first time. The $\mathrm{C}_{16 \mathrm{NH}_{2}}$ Pd NPs are easily accessible and can serve as efficient catalysts for the Suzuki-Miyaura reaction showing very little formation of side product as biphenyl after the completion of the reaction. They are not as active as recently designed molecular palladium catalysts for the Suzuki-Miyaura coupling of arylchlorides, ${ }^{184,} 185$ but they are phosphine-free, easy to synthesize and can be stored as a solid for a long time. The good activity with full product conversion obtained so far open the route to possible modifications of the alkylamine-coated Pd NPs in order to improve these results. The efficiency and usefulness of these alkylamine-coated Pd NPs are already remarkable, however, more study is needed to improve their engineering. 


\section{CHAPTER VII}

\section{SUMMARY, CONCLUSIONS, AND FUTURE DIRECTIONS}

In summary, this dissertation shows the chemical synthesis, characterization, stability, selectivity, and reactivity of $\mathrm{Pd}$ and $\mathrm{Pd}$-containing alloy (PdPt, $\mathrm{PdAu}$ and CuPd) nanoparticles coated with various organic stabilizers. We studied the interaction between hydrogen and Pd and Pd-alloy NPs since it is important for using these NPs as homogenous catalysts for the hydrogenation/isomerization of olefins. Accordingly, we studied the use of these Pd and Pd-alloy NPs as homogeneous catalysts for the hydrogenation/isomerization of various allylic alcohols with different chainlengths for substrate effects. We also explored the thermal stability of various alkylamine-stabilized Pd NPs and studied their catalytic activity for Suzuki-Miyaura reactions.

Chapter II shows the successful synthesis of Pd, PdPt and PdAu NPs stabilized with reduced-L-glutathione under both inert and oxygen atmosphere. We demonstrated that the NPs could be easily prepared under single aqueous phase and confirmed their composition by various analytical techniques. All of these NPs were in the range from 1.1 to $3.7 \mathrm{~nm}$. Increase in the initial mole ratio of glutathione/Pd from 1:3 leads to aggregation of the NPs causing insolubility in the 
aqueous phase. Interestingly, Pd-Glu NPs prepared under oxygen atmosphere showed less ligand coverage than NPs synthesized under inert atmosphere, which was attributed to the formation of $\mathrm{PdO}$ that prohibited the attachment of ligands onto the Pd core surface. The same trends were followed by both PdPt and PdAu NPs stabilized with glutathione synthesized under oxygen and inert atmosphere. All Pd and Pd-alloy NPs stabilized with glutathione were small $(1.1-3.7 \mathrm{~nm})$ and stable. We also demonstrated the successful synthesis of citrate-coated Pd, PdAu and coreshell CuPd NPs under a single aqueous phase. These NPs can be easily prepared and can be used for catalysis after 24 hours. These NPs were highly stable and could be stored in solutions for at least a month. We also synthesized various chain lengths of alkylamine-coated Pd NPs as synthesized before in our lab. ${ }^{75}$ These NPs were in the range of 2.5 to $3.5 \mathrm{~nm}$ in diameter. An increase in the chain-length of $\mathrm{CnNH}_{2}$ in the $\mathrm{CnNH}_{2}$-coated Pd NPs led to small and well-isolated NPs. Pd NPs coated with C16 $\mathrm{NH}_{2}$ ligands with 1:12 mole ratio produced small and stable NPs which was attributed to the formation of multilayers of ligands on the Pd core based on the large percentage of organic content determined by TGA measurements.

Since hydrogen is one of the reactants in the hydrogenation/ isomerization reaction of allyl alcohol, it was important for the synthesized NPs to be stable under a hydrogen atmosphere. In Chapter III, we determined the stability of the aqueous solutions of Pd-Glu (Inert) and Pd-Glu (Oxy) NPs by monitoring UV-Vis spectra of their solutions as a function of exposure time to pure hydrogen bubbling through these solutions. Due to the strong binding of glutathione ligands with Pd NPs, the NPs showed a very similar spectrum for at least 2 hours, confirming that they were 
stable against hydrogen-induced aggregation. The same trend was observed for Pdalloy NPs with Au and Pt with different ratios confirming that glutathione ligands provide stability against hydrogen-induced aggregation. We also studied the stability of Pd-Glu (Inert) NPs and Pd-Glu (Oxy) NPs against hydrogen-induced aggregation under the biphasic condition of chlorobenzene and nanopure water in the presence of allyl alcohol using UV-Vis spectroscopy. UV-Vis spectra showed PdGlu (Inert) to be comparatively more stable than Pd-Glu (Oxy) NPs. For aqueous solutions of Pd-citrate NPs, we determined their hydrogen stability by monitoring the UV-Vis spectra as a function of exposure time to pure hydrogen bubbling through the solution. The presence of hydrogen resulted in the formation of Pd (II) species that probably leached out from the palladium core as seen by a peak at $250 \mathrm{~nm}$ in the UV-Vis spectra. Otherwise, the NPs showed a very similar spectrum with exponential decay in absorbance with an increase in wavelength for an hour, confirming that they were stable against hydrogen-induced aggregation. We also studied the stability of Pd-citrate NPs against hydrogen-induced aggregation under the biphasic condition of dichloromethane and nanopure water in the presence of $\alpha$, $\beta$-unsaturated alcohols (allyl alcohol, 1-penten-3-ol, 1-hepten-3-ol or 2-methyl-3buten-2-ol) using UV-Vis spectroscopy. UV-Vis spectra for Pd-citrate NPs in the presence of allyl alcohol and 1-hepten-3-ol showed low stability and the presence of a high concentration of Pd (II) species formed within the first 5 minutes of the reaction, whereas, Pd-citrate NPs in the presence of 1-penten-3-ol and 2-methyl-3buten-2-ol showed comparatively higher stability with an almost similar spectrum for the first 15 minutes and less formation of Pd (II) species. Overall, this study 
shows that Pd (II) species are formed in the solution with hydrogen both in absence and presence of substrate. This study provides interesting knowledge of hydrogen stability of solutions containing these metal NPs for potential catalysis applications.

Since Suzuki reaction involves heating of the reaction mixture, there was a need to determine the thermal stability of solutions of various chain lengths of alkylamine-stabilized Pd NPs, which can act as potential catalysts using UV-Vis spectroscopy as a function of temperature ranging for 25 to $95^{\circ} \mathrm{C}$. UV-visible spectroscopy showed that the absorbance for $\mathrm{C}^{16 \mathrm{NH}_{2}}$ Pd NPs remains high for all temperatures whereas the absorbance for $\mathrm{C}_{12} \mathrm{NH}_{2} \mathrm{Pd}$ and $\mathrm{C} 8 \mathrm{NH}_{2}$ Pd NPs decreased rapidly at high temperatures which shows temperature-induced aggregation and thus, lower stability of these NPs due to weak interaction of amines with the Pd core. Thus, we decided to use and study $\mathrm{C} 16 \mathrm{NH}_{2}$ Pd NPs as a catalyst for the Suzuki reaction. The $\mathrm{C}_{16 \mathrm{NH}_{2}}$ Pd NPs were tested in toluene solution using UV-Vis spectrum as a function of different temperatures ranging for 30 to $110^{\circ} \mathrm{C}$ each heated for at least 30 minutes. The absorbance remained high for all temperature, showing the

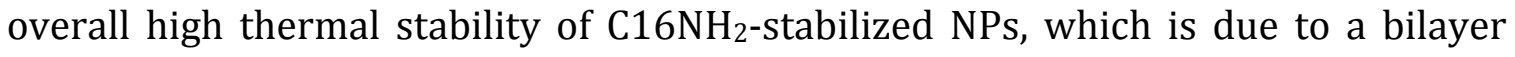
coating of $\mathrm{C}_{16 \mathrm{NH}_{2}}$ ligands on Pd core. ${ }^{75}$ They were thermally suitable for use as a catalyst in the Suzuki reaction.

In Chapter IV, we explored the catalytic activity of various water soluble Pd and Pd-alloy NPs coated with glutathione ligands attached through a thiol functional group for the hydrogenation/isomerization of olefins in a biphasic reaction organic solvent/water mixture. We studied 2-propen-1-ol, the simplest allyl alcohol, as a model substrate first. All of the synthesized NPs catalyzed either or both the 
hydrogenation and isomerization of allyl alcohol with hydrogen in a biphasic solvent mixture to give propanol and/or propanal, respectively. Due to their functionalized end groups and bilateral chains of glutathione, these Pd and Pd-alloy NPs have lower organic-ligand density and thus have the desirable properties of high stability and catalytic activity. However, the reactivity, turnover frequency (TOF), selectivity and recyclability varied depending on the ratio of alloy-mixture composition (100:0, 90:10, 75:25 or 50:50) between Pd and Pt or Au, synthetic environment (inert or aerobic), organic solvent used in biphasic system (dichloromethane, chlorobenzene or ethyl acetate) and the hydrogen flow rates $(8,20$ or $40 \mathrm{~mL} / \mathrm{min})$. We observed that a larger lattice constant resulting from aerobically (oxygen) synthesized NPs making them catalytically more active, but with lower recyclability than the NPs synthesized under inert conditions. Those exhibit high recyclability, but lower TOFs. The ligand attached on the surface of the NPs plays a bigger role than the different lattice constant for product selectivity as all glutathione-coated Pd and Pd-alloy NPs were $80-90 \%$ selective towards the isomerization reaction. The turnover frequency (TOF) increased with increasing hydrogen flow rate. The catalytic activity of various glutathione-coated NPs showed that they followed the trend of lower activity for the monometal composition and more activity as the ratios of alloy metal to $\mathrm{Pd}$ increased. Pd:Au-Glu (Inert/Oxygen) NPs exhibit better TOFs than Pd:Pt-Glu (Inert/Oxygen) NPs with same ratios. This shows that the electronegativity of the metal in the alloy and the ratio of another metal to Pd has a significant role in their overall geometry, allowing more active sites to be available for catalysis. The highest turn over frequency (TOF) for the catalytic reaction was shown by (50:50) Pd:Au- 
Glu (Oxy) NPs with 772 moles of product converted/moles Pd/hour. The selectivity towards hydrogenated and isomerized products after one complete cycle of reaction between allyl alcohol and hydrogen using chlorobenzene over glutathione coated Pd NPs suggested that NPs without alloying with other metals shows higher selectivity towards the isomerized product as compared to alloy NPs. For monometal Pd-Glu NPs, the selectivity remained the same for isomerized product even if hydrogen flow rate is increased. Ethyl acetate solvent in biphasic solvent mixture showed better TOFs than using both chlorobenzene and dichloromethane showing the stability of NPs in these solvents. Biphasic catalysis with chlorobenzene showed higher TOFs when Pd-Glu (Oxygen) NPs were used but lower TOFs when Pd-Glu (Inert) NPs were used, as compare to using dichloromethane as the organic phase. The gluprotected 75:25 Pd:Au NPs synthesized under inert conditions catalyzed the reaction for nine cycles with a $100 \%$ conversion rate in a dichloromethane/ nanopure water biphasic mixture. The branched, open structure of the watersoluble glutathione and use of the biphasic solvent provides high catalytic activity, high selectivity, easy separation of catalyst from the products, and good recyclability.

Following this work, in Chapter IV, we continued with the use of $(75: 25)$ Pd:Au-Glu (Oxy) NPs as a pseudo-homogeneous catalyst for hydrogenation/ isomerization of branched allyl alcohol that differ only slightly in chemical structure in biphasic solvent mixture. While reaction of 1-penten-3-ol in ethyl acetate:water biphasic solvent mixture resulted in a TOF of 317 moles converted/moles Pd/hour, hydrogenation/isomerization reaction of 3-buten-2-ol using same (75:25) Pd:Au- 
Glu (Oxy) NPs with chlorobenzene:water biphasic solvent mixture resulted in a similar TOF of 340 . No products or reactants went to the aqueous phase. The recyclability for 1-penten-3-ol showed one complete cycle with $100 \%$ yield and then it decreased to nearly $52 \%$ after 5 cycles, although the selectivity remained the same for 3-pentanone:3-pentanol (7:3) for all 5 cycles. The recyclability for 3-buten2-ol also showed one complete cycle and then it decreased to nearly $75 \%$ after 5 cycles, with selectivity for 2-butanone:2-butanol (7.5:2.5) for all 5 cycles. The Pd NPs underwent some aggregation after the first cycle, but the selectivity of the catalyst basically remained unchanged over all cycles tested. Thus, we concluded that the more branched substrates had lower reaction rates, likely due to restricted diffusion and access to Pd through the glutathione ligands surrounding the Pd core. The catalytic activity of 2 years old synthesized (75:25) Pd:Au-Glu (Inert) NPs were compared with newly synthesized (75:25) Pd:Au-Glu (Inert) NPs for hydrogenation/isomerization of allyl alcohol. The reactant was converted into products within 10 minutes as observed before, showing high stability of glutathione-coated NPs. The only difference observed was that \% hydrogenation for 1-propanol increased from $16 \%$ to $33 \%$, whereas $\%$ isomerization for 1-propanal decreased from $84 \%$ to $67 \%$, which could be due to the loss of some ligands from the Pd surface with time.

In chapter V, we examined the catalytic activity of water-soluble Pd-citrate NPs that show high stability under a biphasic solvent system and high catalytic performances with better turnover frequencies (TOFs) towards the hydrogenation/ isomerization of various chain-lengths of $\alpha, \beta$-unsaturated alcohols to form 
hydrogenated and isomerized products and recyclability with $100 \%$ product conversion based on the substrate structure for the first time. Pd-citrate NPs showed higher stability under dichloromethane organic solvent than ethyl acetate and chlorobenzene solvents. Pd NPs also showed high stability under a constant flow of hydrogen gas for at least 1 hour according to UV/Vis-spectroscopy, which was necessary for the catalytic reaction. The "naked" Pd-citrate NPs were highly efficient in catalyzing various long chain $\alpha, \beta$-unsaturated alcohols with high turnover rates, with the highest being 8870 moles product/moles Pd/hour for 1penten-3-ol, 6114 for 1-hepten-3-ol and 4452 for allyl alcohol. These are about an order of magnitude higher than other Pd NPs reported in the literature. The recyclability was 5 times for 1-penten-3-ol, 3 times for allyl alcohol and 2 times for 1-hepten-3-ol with $100 \%$ product conversion. Thus, the substrate structure plays an important role in the catalytic activity of the NPs, resulting in 1-penten-3-ol being catalytically more active than allyl alcohol and 1-hepten-3-ol due to its appropriate hydrocarbon chain length that facilitates the orientation of the substrate active site towards the catalyst present in the aqueous phase. The Pd-citrate NPs have lower TOF and lower recyclability for 2-methyl-3-buten-2-ol when compared with 1penten-3-ol with the same number of carbon atoms due to high steric hindrance causing lower stability of Pd NPs. This confirms that straight chain substrates are catalytically more active than branched substrate with the same number of carbon atoms. Also, the use of a biphasic solvent system was observed to be more efficient than using a mono-phasic aqueous solvent system for the hydrogenation/ isomerization reaction of unsaturated alcohols since presence of organic phase acts 
as gates providing just enough substrate molecules to interact with the Pd-citrate NPs and thus provide higher stability than monophasic. Thus, biphasic catalysis shows high NPs stability, TOF and high recyclability with $100 \%$ product conversion. The selectivity of Pd-citrate NPs towards hydrogenated and isomerized products after 1 complete cycle of reaction for all substrates was 3:2. We also synthesized citrate-capped CuPd core-shell NPs and used them for the hydrogenation/isomerization of 1-penten-3-ol. Interestingly, CuPd-citrate NPs showed a high selectivity with $\sim 95 \%$ preference for hydrogenated product, although with much lower TOF compared to Pd-citrate NPs. Thus, due to their high accessibility, well-defined surface morphology and high stability in the biphasic solution, Pd-citrate NPs show great promise as convenient and active catalysts.

In Chapter VI we explored the catalytic properties of alkylamine-coated Pd NPs for the C-C cross coupling Suzuki-Miyaura reaction of arylhalide with aryl boronic acids to form unsymmetrical biaryls. Thermal stability data in toluene solvent using UV-Vis spectroscopy as a function of different temperatures shows high stability of $\mathrm{C}^{16 \mathrm{NH}_{2}}$ Pd NPs with high absorbance even under high temperatures of $110^{\circ} \mathrm{C}$ due to the bilayer coating of $\mathrm{C} 16 \mathrm{NH}_{2}$ ligands on $\mathrm{Pd}$ core. $\mathrm{C} \mathrm{NH}_{2} \mathrm{Pd}$ and $\mathrm{C}_{12} \mathrm{NH}_{2} \mathrm{Pd}$ aggregated at high temperatures and thus, couldn't be used for reactions involving high temperatures. Thus, we decided to use and study $\mathrm{C}_{16} \mathrm{NH}_{2}$ Pd NPs as a catalyst for the Suzuki-Miyaura reaction. We choose the model Suzuki reaction which invloves the mixing of 4-iodoanisole and phenylboronic acid and Pd NPs catalyst in a toluene:water biphasic mixture with potassium carbonate as base dissolved in a minimal amount of water. The reaction was heated to $70^{\circ} \mathrm{C}$. 
The reaction completed with all of limiting reagent being consumed within 4 hours. After completion of the reaction, product formation was confirmed with TLC and GC-MS. Both show completion of the Suzuki reaction with the formation of product and little side product (biphenyl) due to homo coupling of phenylboronic acid. $\mathrm{C}_{16 \mathrm{NH}_{2}} \mathrm{Pd} \mathrm{NPs}$ used for this reaction as catalyst aggregated after the completion of the reaction. Further research is going on to add primary reactants (4-iodoanisole and pheylboronic acid) as solution in organic solvent drop wise in the reaction solution to obtain the products while keeping the catalyst system stabilized. More work is needed to solve this problem of aggregation by adding solution of both primary reactants in toluene drop wise so as to stop the aggregation of NPs, but still this study has led to interesting insights regarding the effects of various reaction parameters and choice of alkylamine as ligand for Suzuki-Miyaura Pd catalysts.

Overall, this work shows that metal nanoparticles can be easily synthesized and their functionality and composition can be tailored to impart useful properties for catalysis applications. Variations in the type of ligands, different synthetic environment leading to different lattice parameters, metal composition, hydrogen flow rates, different organic solvents in biphasic solvent reaction mixture and various substrate structures had drastic effects on the stability, reactivity and selectivity of these NPs. The chemistry of these materials can be altered to optimize their performances by designing stable, highly reactive, selective, recyclable and efficient catalysts. It will also pave new avenues in the development of choosing better candidates for hydrogen sensing and storage. In addition, we envision that this study could provide a powerful platform for developing novel nanostructured 
materials with controlled parameters, which can be used in a range of nanocatalytic applications. Taken together, this study will have a great impact in the field of catalysis and sensing.

\section{FUTURE DIRECTIONS:}

Future research should focus on modifying the ligands that stabilize the Pd and Pd-alloy NPs. This should be further explored to design strategies that improve the efficiency and reactivity of the NPs over various consecutive cycles. The ligands could have a coating on the Pd core as a cage structure where substrate can easily go inside to react at the Pd surface and products could be easily removed, leaving behind the Pd core intact. It is also important to study the use of these organicstabilized Pd and Pd-alloy NPs as catalysts for other C-C coupling reactions like Heck reaction and Sonogashira reactions, which are also very important class of organic reactions with industrial applications other than Suzuki reactions. It would be also interesting to explore different synthesis methods for increasing the copper content in Cu@Pd NPs while still maintaining high stability, catalytic activity, and high selectivity.

The search for various ligands allowing high stability, reactivity, selectivity and recyclability will be an ongoing challenge. 


\section{REFERENCES}

1. Dotzauer, D. M.; Bhattacharjee, S.; Wen, Y.; Bruening, M. L. Langmuir 2009, 25, (3), 1865-1871.

2. Lin, Y.; Watson, K. A.; Fallbach, M. J.; Ghose, S.; Smith, J. G.; Delozier, D. M.; Cao, W.; Crooks, R. E.; Connell, J. W. ACS Nano 2009, 3, (4), 871-884.

3. Narayanan, R.; El-Sayed, M. A. J. Catal. 2005, 234, (2), 348-355.

4. $\quad$ Astruc, D.; Lu, F.; Aranzaes, J. R. Angew. Chem. Int. Ed. 2005, 44, (48), 78527872.

5. Roucoux, A.; Schulz, J.; Patin, H. Chem. Rev. 2002, 102, (10), 3757-3778.

6. $\quad$ Durand, J.; Teuma, E.; Gómez, M. Eur. J. Inorg. Chem. 2008, 2008, (23), 35773586.

7. Brust, M.; Walker, M.; Bethell, D.; Schiffrin, D. J.; Whyman, R. J. Chem. Soc., Chem. Commun. 1994, (7), 801-802.

8. Scott, R. W. J.; Wilson, O. M.; Crooks, R. M. J. Phys. Chem. B 2005, 109, (2), 692704.

9. Shon, Y.-S.; Chuc, S.; Voundi, P. Colloids Surf., A 2009, 352, (1), 12-17.

10. Chen, S.; Yang, Y.J. Am. Chem. Soc. 2002, 124, (19), 5280-5281.

11. Cao, Y. C.; Jin, R.; Mirkin, C. A. Science 2002, 297, (5586), 1536.

12. Kamat, P. V. J. Phys. Chem. B 2002, 106, (32), 7729-7744.

13. Ohara, S.; Hatakeyama, Y.; Umetsu, M.; Sato, K.; Naka, T.; Adschiri, T. J. Power Sources 2009, 193, (1), 367-370.

14. Ibañez, F. J.; Zamborini, F. P. Small 2012, 8, (2), 174-202.

15. Andres, R. P.; Bein, T.; Dorogi, M.; Feng, S.; Henderson, J. I.; Kubiak, C. P.; Mahoney, W.; Osifchin, R. G.; Reifenberger, R. Science 1996, 272, (5266), 1323. 
16. Schwartzberg, A. M.; Zhang, J. Z. J. Phys. Chem. C 2008, 112, (28), 1032310337.

17. Hicks, J. F.; Zamborini, F. P.; Murray, R. W. J. Phys. Chem. B 2002, 106, (32), 7751-7757.

18. Lee, D.; Donkers, R. L.; Wang, G.; Harper, A. S.; Murray, R. W. J. Am. Chem. Soc. 2004, 126, (19), 6193-6199.

19. Ristau, R.; Tiruvalam, R.; Clasen, P. L.; Gorskowski, E. P.; Harmer, M. P.; Kiely, C. J.; Hussain, I.; Brust, M. Gold Bull. 2009, 42, (2), 133-143.

20. Gréget, R.; Nealon, G. L.; Vileno, B.; Turek, P.; Mény, C.; Ott, F.; Derory, A.; Voirin, E.; Rivière, E.; Rogalev, A.; Wilhelm, F.; Joly, L.; Knafo, W.; Ballon, G.; Terazzi, E.; Kappler, J.-P.; Donnio, B.; Gallani, J.-L. ChemPhysChem 2012, 13, (13), 3092-3097.

21. Mandal, S.; Roy, D.; Chaudhari, R. V.; Sastry, M. Chem. Mater. 2004, 16, (19), 3714-3724.

22. Crooks, R. M.; Zhao, M.; Sun, L.; Chechik, V.; Yeung, L. K. Acc. Chem. Res. 2001, 34, (3), 181-190.

23. Sardar, R.; Funston, A. M.; Mulvaney, P.; Murray, R. W. Langmuir 2009, 25, (24), 13840-13851.

24. Chen, M.; Goodman, D. W. Acc. Chem. Res. 2006, 39, (10), 739-746.

25. Chen, M.; Goodman, D. W. Chem. Soc. Rev. 2008, 37, (9), 1860-1870.

26. Bönnemann, H.; Richards, Ryan M. Eur. J. Inorg. Chem. 2001, 2001, (10), 2455-2480.

27. Chen, H. M.; Liu, R.-S. J. Phys. Chem. C 2011, 115, (9), 3513-3527.

28. Bell, I. R.; Schwartz, G. E. Homeopathy 104, (2), 123-138.

29. Mizukoshi, Y.; Okitsu, K.; Maeda, Y.; Yamamoto, T. A.; Oshima, R.; Nagata, Y. J. Phys. Chem. B 1997, 101, (36), 7033-7037.

30. Giorgio, S.; Chapon, C.; Henry, C. R. Langmuir 1997, 13, (8), 2279-2284.

31. Bradley, J.-C.; Ma, Z. Angew. Chem. Int. Ed. 1999, 38, (11), 1663-1666.

32. Alvarez, J.; Liu, J.; Roman, E.; Kaifer, A. E. Chem. Commun. 2000, (13), 11511152.

33. Castro, E. G.; Salvatierra, R. V.; Schreiner, W. H.; Oliveira, M. M.; Zarbin, A. J. G. Chem. Mater. 2010, 22, (2), 360-370. 
34. Eklund, S. E.; Cliffel, D. E. Langmuir 2004, 20, (14), 6012-6018.

35. Li, Z.; Gao, J.; Xing, X.; Wu, S.; Shuang, S.; Dong, C.; Paau, M. C.; Choi, M. M. F. J. Phys. Chem. C 2010, 114, (2), 723-733.

36. Lu, F.; Ruiz, J.; Astruc, D. Tetrahedron Lett. 2004, 45, (51), 9443-9445.

37. Şen, F.; Gökağaç, G. J. Phys. Chem. C 2007, 111, (15), 5715-5720.

38. Zamborini, F. P.; Gross, S. M.; Murray, R. W. Langmuir 2001, 17, (2), 481-488.

39. Alkilany, A. M.; Murphy, C. J. Langmuir 2009, 25, (24), 13874-13879.

40. Cliffel, D. E.; Zamborini, F. P.; Gross, S. M.; Murray, R. W. Langmuir 2000, 16, (25), 9699-9702.

41. Ma, R.; Semagina, N. J. Phys. Chem. C 2010, 114, (36), 15417-15423.

42. Chauhan, B. P. S.; Rathore, J. S.; Bandoo, T. J. Am. Chem. Soc. 2004, 126, (27), 8493-8500.

43. Li, Y.; El-Sayed, M. A. J. Phys. Chem. B 2001, 105, (37), 8938-8943.

44. Ma, L.; Abney, C.; Lin, W. Chem. Soc. Rev. 2009, 38, (5), 1248-1256.

45. Narayanan, R.; El-Sayed, M. A. J. Am. Chem. Soc. 2003, 125, (27), 8340-8347.

46. Turkevich, J.; Stevenson, P. C.; Hillier, J. Spec. Discuss. Faraday Soc. 1951, 11, (0), 55-75.

47. Henglein, A. J. Phys. Chem. B 2000, 104, (29), 6683 - 6685.

48. Huang, Y.-X.; Xie, J.-F.; Zhang, X.; Xiong, L.; Yu, H.-Q. ACS Appl. Mater. Interfaces 2014, 6, (18), 15795 - 15801.

49. Wilson, O. M.; Knecht, M. R.; Garcia-Martinez, J. C.; Crooks, R. M. J. Am. Chem. Soc. 2006, 128, (14), 4510-4511.

50. Ooe, M.; Murata, M.; Mizugaki, T.; Ebitani, K.; Kaneda, K. Nano Lett. 2002, 2, (9), 999-1002.

51. Tsuji, Y.; Fujihara, T. Inorg. Chem. 2007, 46, (6), 1895-1902.

52. Jiang, Y.; Gao, Q.J. Am. Chem. Soc. 2006, 128, (3), 716-717.

53. Mayer, A. B. R.; Mark, J. E.; Hausner, S. H. Angew. makromol. chem. 1998, 259, (1), 45-53.

54. Mayer, A. B. R.; Mark, J. E. J. Polym. Sci., Part A: Polym. Chem. 1997, 35, (15), 3151-3160.

55. Mayer, A. B. R.; Hausner, S. H.; Mark, J. E. Polym. J. 2000, 32, (1), 15-22. 
56. Mayer, A.; Antonietti, M. Colloid. Polym. Sci. 1998, 276, (9), 769-779.

57. Garcia-Martinez, J. C.; Lezutekong, R.; Crooks, R. M. J. Am. Chem. Soc. 2005, 127, (14), 5097-5103.

58. Chechik, V.; Crooks, R. M. J. Am. Chem. Soc. 2000, 122, (6), 1243-1244.

59. Ibañez, F. J.; Zamborini, F. P. ACS Nano 2008, 2, (8), 1543-1552.

60. Ibañez, F. J.; Zamborini, F. P. J. Am. Chem. Soc. 2008, 130, (2), 622-633.

61. Ibañez, F. J.; Zamborini, F. P. Langmuir 2006, 22, (23), 9789-9796.

62. Nakao, Y.; Kaeriyama, K. J. Colloid Interface Sci. 1986, 110, (1), 82-87.

63. Pasquato, L.; Pengo, P.; Scrimin, P. J. Mater. Chem. 2004, 14, (24), 3481-3487.

64. Porter, L. A.; Ji, D.; Westcott, S. L.; Graupe, M.; Czernuszewicz, R. S.; Halas, N. J.;

Lee, T. R. Langmuir 1998, 14, (26), 7378-7386.

65. Templeton, A. C.; Wuelfing, W. P.; Murray, R. W. Acc. Chem. Res. 2000, 33, (1), 27-36.

66. Hostetler, M. J.; Wingate, J. E.; Zhong, C.-J.; Harris, J. E.; Vachet, R. W.; Clark, M. R.; Londono, J. D.; Green, S. J.; Stokes, J. J.; Wignall, G. D.; Glish, G. L.; Porter, M. D.; Evans, N. D.; Murray, R. W. Langmuir 1998, 14, (1), 17-30.

67. Templeton, A. C.; Hostetler, M. J.; Warmoth, E. K.; Chen, S.; Hartshorn, C. M.; Krishnamurthy, V. M.; Forbes, M. D. E.; Murray, R. W. Journal of the American Chemical Society 1998, 120, (19), 4845-4849.

68. Yee, C.; Scotti, M.; Ulman, A.; White, H.; Rafailovich, M.; Sokolov, J. Langmuir 1999, 15, (13), 4314-4316.

69. He, S.; Yao, J.; Jiang, P.; Shi, D.; Zhang, H.; Xie, S.; Pang, S.; Gao, H. Langmuir 2001, 17, (5), 1571-1575.

70. Zamborini, F. P.; Leopold, M. C.; Hicks, J. F.; Kulesza, P. J.; Malik, M. A.; Murray, R. W. Journal of the American Chemical Society 2002, 124, (30), 8958-8964.

71. Ganesan, M.; Freemantle, R. G.; Obare, S. O. Chemistry of Materials 2007, 19, (14), 3464-3471.

72. Shon, Y.-S.; Wuelfing, W. P.; Murray, R. W. Langmuir 2001, 17, (4), 12551261.

73. Kumar, A.; Mandal, S.; Selvakannan, P. R.; Pasricha, R.; Mandale, A. B.; Sastry, M. Langmuir 2003, 19, (15), 6277-6282. 
74. Moreno, M.; Ibañez, F. J.; Jasinski, J. B.; Zamborini, F. P. J. Am. Chem. Soc. 2011, 133, (12), 4389-4397.

75. Moreno, M.; Kissell, L. N.; Jasinski, J. B.; Zamborini, F. P. ACS Catal. 2012, 2, (12), 2602-2613.

76. Leff, D. V.; Brandt, L.; Heath, J. R. Langmuir 1996, 12, (20), 4723-4730.

77. Rao, C. R. K.; Lakshminarayanan, V.; Trivedi, D. C. Materials Letters 2006, 60, (25), 3165-3169.

78. Joseph, Y.; Guse, B.; Yasuda, A.; Vossmeyer, T. Sensors and Actuators B: Chemical 2004, 98, (2), 188-195.

79. Cargnello, M.; Wieder, N. L.; Canton, P.; Montini, T.; Giambastiani, G.; Benedetti, A.; Gorte, R. J.; Fornasiero, P. Chem. Mater. 2011, 23, (17), 3961-3969.

80. Sadeghmoghaddam, E.; Lam, C.; Choi, D.; Shon, Y.-S. J. Mater. Chem. 2011, 21, (2), 307-312.

81. Sadeghmoghaddam, E.; Gaïeb, K.; Shon, Y.-S. Appl. Catal., A 2011, 405, (1-2), 137-141.

82. J. Dispersion Sci. Technol. 2005, 26, (3), 389-414.

83. Kim, K.; Kim, K. L.; Shin, K. S. J. Phys. Chem. C 2011, 115, (30), 14844 - 14851.

84. Han, J.; Zhou, Z.; Yin, Y.; Luo, X.; Li, J.; Zhang, H.; Yang, B. CrystEngComm 2012, 14, (20), $7036-7042,7$.

85. Slouf, M.; Pavlova, E.; Bhardwaj, M.; Plestil, J.; Onderkova, H.; Philimonenko, A. A.; Hozak, P. Mater. Lett. 2011, 65, (8), 1197 - 1200.

86. Musolino, M. G.; De Maio, P.; Donato, A.; Pietropaolo, R. J. Mol. Catal. A: Chem. 2004, 208, (1), 219-224.

87. Musolino, M. G.; Cutrupi, C. M. S.; Donato, A.; Pietropaolo, D.; Pietropaolo, R. Appl. Catal., A 2003, 243, (2), 333-346.

88. Bhattacharjee, S.; Dotzauer, D. M.; Bruening, M. L. J. Am. Chem. Soc. 2009, 131, (10), 3601 - 3610.

89. Bhattacharjee, S.; Bruening, M. L. Langmuir 2008, 24, (6), 2916-2920.

90. Kidambi, S.; Dai, J.; Li, J.; Bruening, M. L. J. Am. Chem. Soc. 2004, 126, (9), 2658-2659.

91. Kidambi, S.; Bruening, M. L. Chem. Mater. 2005, 17, (2), 301-307. 
92. Zharmagambetova, A. K.; Ergozhin, E. E.; Sheludyakov, Y. L.; Mukhamedzhanova, S. G.; Kurmanbayeva, I. A.; Selenova, B. A.; Utkelov, B. A. J. Mol. Catal. A: Chem. 2001, 177, (1), 165-170.

93. Niu, Y.; Yeung, L. K.; Crooks, R. M. J. Am. Chem. Soc. 2001, 123, (28), 68406846.

94. Oh, S.-K.; Niu, Y.; Crooks, R. M. Langmuir 2005, 21, (22), 10209-10213.

95. Yeung, L. K.; Crooks, R. M. Nano Lett. 2001, 1, (1), 14-17.

96. Scott, R. W. J.; Datye, A. K.; Crooks, R. M. J. Am. Chem. Soc. 2003, 125, (13), 3708-3709.

97. Maung, M. S.; Dinh, T.; Salazar, C.; Shon, Y.-S. Colloids Surf., A 2017, 513, 367372.

98. Hostetler, M. J.; Templeton, A. C.; Murray, R. W. Langmuir 1999, 15, (11), 3782-3789.

99. Kye Jin, J.; Jun Min, L.; Eunsongyi, L.; Wooyoung, L. Nanotechnology 2009, 20, (13), 135502.

100. Jia, W.; Su, L.; Ding, Y.; Schempf, A.; Wang, Y.; Lei, Y. J. Phys. Chem. C 2009, 113, (37), 16402-16407.

101. Claussen, J. C.; Franklin, A. D.; ul Haque, A.; Porterfield, D. M.; Fisher, T. S. ACS Nano 2009, 3, (1), 37-44.

102. Favier, F.; Walter, E. C.; Zach, M. P.; Benter, T.; Penner, R. M. Science 2001, 293, (5538), 2227.

103. Sun, Y.; Wang, H. H.; Xia, M. The Journal of Physical Chemistry C 2008, 112, (4), 1250-1259.

104. Lewis, F. A., The palladium hydrogen system. Academic Press: London, 1967.

105. RaviPrakash, J.; McDaniel, A. H.; Horn, M.; Pilione, L.; Sunal, P.; Messier, R.; McGrath, R. T.; Schweighardt, F. K. Sens. Actuators, B 2007, 120, (2), 439-446.

106. Hakamada, M.; Nakano, H.; Furukawa, T.; Takahashi, M.; Mabuchi, M. J. Phys. Chem. C 2010, 114, (2), 868-873.

107. Sandrock, G. J. Alloys Compd. 1999, 293-295, 877-888.

108. Smith, A. L.; Shirazi, H. M. Thermochim. Acta 2005, 432, (2), 202-211.

109. Yang, F.; Taggart, D. K.; Penner, R. M. Nano Lett. 2009, 9, (5), 2177-2182. 
110. Yu, S.; Welp, U.; Hua, L. Z.; Rydh, A.; Kwok, W. K.; Wang, H. H. Chem. Mater. 2005, 17, (13), 3445-3450.

111. Dasari, R.; Zamborini, F. P. J. Am. Chem. Soc. 2008, 130, (48), 16138-16139.

112. Robinson, J. A.; Snow, E. S.; Bădescu, Ş. C.; Reinecke, T. L.; Perkins, F. K. Nano Lett. 2006, 6, (8), 1747-1751.

113. Yun, M.; Myung, N. V.; Vasquez, R. P.; Lee, C.; Menke, E.; Penner, R. M. Nano Lett. 2004, 4, (3), 419-422.

114. Kolmakov, A.; Klenov, D. O.; Lilach, Y.; Stemmer, S.; Moskovits, M. Nano Lett. 2005, 5, (4), 667-673.

115. Staii, C.; Johnson, A. T.; Chen, M.; Gelperin, A. Nano Lett. 2005, 5, (9), 17741778.

116. Kaltenpoth, G.; Schnabel, P.; Menke, E.; Walter, E. C.; Grunze, M.; Penner, R. M. Anal. Chem. 2003, 75, (18), 4756-4765.

117. Luongo, K.; Sine, A.; Bhansali, S. Sens. Actuators, B 2005, 111, 125-129.

118. Walter, E. C.; Favier, F.; Penner, R. M. Anal. Chem. 2002, 74, (7), 1546-1553.

119. Lin, H.; Gao, T.; Fantini, J.; Sailor, M. J. Langmuir 2004, 20, (12), 5104-5108.

120. Zhao, Z.; Sevryugina, Y.; Carpenter, M. A.; Welch, D.; Xia, H. Anal. Chem. 2004, 76, (21), 6321-6326.

121. Zhao, Z.; Carpenter, M. A. J. Appl. Phys. 2005, 97, (12), 124301.

122. Wagener, P.; Schwenke, A.; Barcikowski, S. Langmuir 2012, 28, (14), 61326140 .

123. Panigrahi, S.; Basu, S.; Praharaj, S.; Pande, S.; Jana, S.; Pal, A.; Ghosh, S. K.; Pal, T. J. Phys. Chem. C 2007, 111, (12), 4596-4605.

124. Narayanan, R.; El-Sayed, M. Top. Catal. 2008, 47, (1-2), 15-21.

125. Semagina, N.; Kiwi-Minsker, L. Catal. Rev. 2009, 51, (2), 147-217.

126. Gavia, D. J.; Maung, M. S.; Shon, Y.-S. ACS Appl. Mater. Interfaces 2013, 5, (23), 12432-12440.

127. Esumi, K.; Sarashina, S.; Yoshimura, T. Langmuir 2004, 20, (13), 5189-5191.

128. Isaacs, S. R.; Cutler, E. C.; Park, J.-S.; Lee, T. R.; Shon, Y.-S. Langmuir 2005, 21, (13), 5689-5692. 
129. Negishi, Y.; Kurashige, W.; Kamimura, U. Langmuir 2011, 27, (20), 1228912292.

130. Horinouchi, S.; Yamanoi, Y.; Yonezawa, T.; Mouri, T.; Nishihara, H. Langmuir 2006, 22, (4), 1880-1884.

131. Murphy, P. B.; Liu, F.; Cook, S. C.; Jahan, N.; Marangoni, D. G.; Grindley, T. B.; Zhang, P. Can. J. Chem. 2009, 87, (11), 1641-1649.

132. Sadeghmoghaddam, E.; Gu, H.; Shon, Y.-S. ACS Catal. 2012, 2, (9), 1838-1845.

133. Gavia, D. J.; Shon, Y.-S. Langmuir 2012, 28, (40), 14502-14508.

134. Marshall, S. T.; O’Brien, M.; Oetter, B.; Corpuz, A.; Richards, R. M.; Schwartz, D. K.; Medlin, J. W. Nat. Mater. 2010, 9, (10), 853-858.

135. Weng, Z.; Zaera, F. J. Phys. Chem. C 2014, 118, (7), 3672-3679.

136. Lee, J.; Shim, H. S.; Lee, M.; Song, J. K.; Lee, D. J. Phys. Chem. Lett. 2011, 2, (22), 2840-2845.

137. Wu, Q.; Cao, H.; Luan, Q.; Zhang, J.; Wang, Z.; Warner, J. H.; A. R. Watt, A. Inorg. Chem. 2008, 47, (13), 5882-5888.

138. Wu, Z.; Chen, J.; Jin, R. Adv. Funct. Mater. 2011, 21, (1), 177-183.

139. Briñas, R. P.; Hu, M.; Qian, L.; Lymar, E. S.; Hainfeld, J. F. J. Am. Chem. Soc. 2008, 130, (3), 975-982.

140. Yuan, X.; Luo, Z.; Zhang, Q.; Zhang, X.; Zheng, Y.; Lee, J. Y.; Xie, J. ACS Nano 2011, 5, (11), 8800-8808.

141. Baruwati, B.; Polshettiwar, V.; Varma, R. S. Green Chem. 2009, 11, (7), 926930.

142. Ben Moshe, A.; Markovich, G. Chem. Mater. 2011, 23, (5), 1239-1245.

143. Sharma, S.; Kim, B.; Lee, D. Langmuir 2012, 28, (45), 15958-15965.

144. Miao, S.; Zhang, C.; Liu, Z.; Han, B.; Xie, Y.; Ding, S.; Yang, Z. J. Phys. Chem. C 2008, 112, (3), 774-780.

145. Wu, H.; Wu, C.; He, Q.; Liao, X.; Shi, B. Mater. Sci. Eng. C. 2010, 30, (5), 770776.

146. Ornelas, C.; Aranzaes, J. R.; Salmon, L.; Astruc, D. Chem. Eur. J. 2008, 14, (1), $50-64$. 
147. Ornelas, C.; Diallo, A. K.; Ruiz, J.; Astruc, D. Adv. Synth. Catal. 2009, 351, (13), 2147-2154.

148. Huang, X.; Wang, Y.; He, Q.; Liao, X.; Shi, B. Catal. Lett. 2009, 133, (1), 192.

149. Yijun, J.; Jinhua, J.; Qiuming, G.; Meiling, R.; Himei, Y.; Lingjun, Q. Nanotechnology 2008, 19, (7), 075714.

150. Polshettiwar, V.; Luque, R.; Fihri, A.; Zhu, H.; Bouhrara, M.; Basset, J.-M. Chem. Rev. 2011, 111, (5), 3036-3075.

151. Wang, Y.; Lee, J.-K. J. Mol. Catal. A: Chem. 2007, 263, (1), 163-168.

152. Lorenzo-Luis, P.; Romerosa, A.; Serrano-Ruiz, M. ACS Catal. 2012, 2, (6), 1079-1086.

153. Sabitha, G.; Nayak, S.; Bhikshapathi, M.; Yadav, J. S. Org. Lett. 2011, 13, (3), 382-385.

154. Uma, R.; Crévisy, C.; Grée, R. Chem. Rev. 2003, 103, (1), 27-52.

155. Ito, M.; Kitahara, S.; Ikariya, T. J. Am. Chem. Soc. 2005, 127, (17), 6172-6173.

156. Bouziane, A.; Régnier, T.; Carreaux, F.; Carboni, B.; Bruneau, C.; Renaud, J.-L. Synlett 2010, 2010, (02), 207-210.

157. Bovo, S.; Scrivanti, A.; Bertoldini, M.; Beghetto, V.; Matteoli, U. Synthesis 2008, 2008, (16), 2547-2550.

158. Tanaka, N.; Suzuki, T.; Matsumura, T.; Hosoya, Y.; Nakada, M. Angew. Chem. Int. Ed. 2009, 48, (14), 2580-2583.

159. van der Drift, R. C.; Bouwman, E.; Drent, E. J. Organomet. Chem. 2002, 650, (1), 1-24.

160. McGrath, D. V.; Grubbs, R. H. Organometallics 1994, 13, (1), 224-235.

161. Gavia, D. J.; Maung, M. S.; Shon, Y.-S. ACS Appl. Mater. Interfaces 2013, 5, (23), 12432 - 12440.

162. Miyaura, N.; Suzuki, A. Chem. Rev. 1995, 95, (7), 2457-2483.

163. Suzuki, A. J. Organomet. Chem. 1999, 576, (1), 147-168.

164. Wolfe, J. P.; Singer, R. A.; Yang, B. H.; Buchwald, S. L. J. Am. Chem. Soc. 1999, 121, (41), 9550-9561.

165. Miyaura, N.; Yamada, K.; Suzuki, A. Tetrahedron Lett. 1979, 20, (36), 34373440 . 
166. Torborg, C.; Beller, M. Adv. Synth. Catal. 2009, 351, (18), 3027-3043.

167. Magano, J.; Dunetz, J. R. Chem. Rev. 2011, 111, (3), 2177-2250.

168. Suzuki, A. VCH: Weinheim 1998, 49-97.

169. Bradley J. S., S., G. Ed.; VCH: Weinheim 1994, 523-536.

170. Reetz, M. T.; Breinbauer, R.; Wanninger, K. Tetrahedron Lett. 1996, 37, (26), 4499-4502.

171. Li, Y.; Hong, X. M.; Collard, D. M.; El-Sayed, M. A. Org. Lett. 2000, 2, (15), 23852388.

172. Narayanan, R.; El-Sayed, M. A. J. Phys. Chem. B 2004, 108, (25), 8572-8580.

173. Narayanan, R.; El-Sayed, M. A. Langmuir 2005, 21, (5), 2027-2033.

174. Gopidas, K. R.; Whitesell, J. K.; Fox, M. A. Nano Lett. 2003, 3, (12), 1757-1760.

175. Strimbu, L.; Liu, J.; Kaifer, A. E. Langmuir 2003, 19, (2), 483-485.

176. Kogan, V.; Aizenshtat, Z.; Popovitz-Biro, R.; Neumann, R. Org. Lett. 2002, 4, (20), 3529-3532.

177. Gaikwad, A. V.; Holuigue, A.; Thathagar, M. B.; ten Elshof, J. E.; Rothenberg, G. Chem. Eur. J. 2007, 13, (24), 6908-6913.

178. Zhang, J.; Xu, X.; Yang, C.; Yang, F.; Yang, X. Anal. Chem. 2011, 83, (10), 39113917.

179. Park, J.-W.; Shumaker-Parry, J. S. ACS Nano 2015, 9, (2), 1665-1682.

180. Cadierno, V.; García-Garrido, S. E.; Gimeno, J.; Varela-Álvarez, A.; Sordo, J. A. J. Am. Chem. Soc. 2006, 128, (4), 1360-1370.

181. Scott, R. W. J.; Wilson, O. M.; Oh, S.-K.; Kenik, E. A.; Crooks, R. M. J. Am. Chem. Soc. 2004, 126, (47), 15583-15591.

182. Musolino; De, M.; Donato; Pietropaolo. J. Mol. Catal. A: Chem. 2004, 208, (1-2), $219-224$.

183. Calver, C. F.; Dash, P.; Scott, R. W. J. ChemCatChem 2011, 3, (4), 695-697.

184. Miura, M. Angew. Chem. Int. Ed. 2004, 43, (17), 2201-2203.

185. Littke, A. F.; Fu, G. C. Angew. Chem. Int. Ed. 2002, 41, (22), 4176-4211. 


\title{
CURRICULUM VITAE
}

\author{
Shekhar Bhama \\ Department of Chemistry \\ University of Louisville \\ Louisville, KY, USA 40292 \\ Telephone: (502) 852-5982 \\ E-mail: bhama43@gmail.com
}

\section{PROFESSIONAL OBJECTIVE:}

To utilize my research experience in Analytical (Material Science) Chemistry with emphasis on designing and synthesis of new capping ligands and nanoparticles for the homogenous and heterogeneous nano-catalysis and contribute to the research and development processes in a research facility. Moreover, I feel confident that I can pursue research in modern interdisciplinary and multidisciplinary fields; especially in the field of synthetic organic chemistry, packaging materials analysis, distillation processes and surface-enhanced raman techniques and can bring the best of my abilities. 


\section{EDUCATIONAL BACKGROUND:}

Ph.D. in Analytical Chemistry (Material Science)

2012 to 2017

University of Louisville, Louisville, KY, USA

Advisor: Dr. Francis P. Zamborini

(Professor, Director of Graduate Studies)

M.S. in Inorganic Chemistry (Honors)

2005 to 2007

Panjab University, Chandigarh, India

Advisor: Dr. Rajpal Sharma (Professor)

B.S. in Industrial Chemistry

2002 to 2005

G.G.D.S.D. College, Panjab University, India

\section{RESEARCH EXPERIENCE:}

Graduate Research Assistant, Department of Chemistry, University of Louisville, 2012 - August 2017

1. Completed 5 research projects by synthesizing $\mathrm{Pd}$ and $\mathrm{Pd}$-alloy $(\mathrm{Au}, \mathrm{Pt}$ and $\mathrm{Cu})$ monolayer protected clusters (MPCs)/nanoparticles (NPs) coated with various capping ligands.

2. Designed various ligands that can stabilize the nanoparticles while keeping the catalyst active and characterizing them using TEM, EDAX, XRD, XPS, AFM, TGA, ${ }^{1} \mathrm{H}-\mathrm{NMR}, \mathrm{FT}-\mathrm{IR}, \mathrm{GC}-\mathrm{MS}$.

3. Modified the surface composition of metal nanoparticles to increase the catalytic activity, selectivity and recyclability.

4. Evaluated the reactivity between hydrogen and the MPCs by controlling the metal and ligand ratio composition of the nanoparticles for hydrogen sensing, storage and catalysis applications. 
5. Explored the catalytic properties of NPs for the hydrogenation and isomerization of various allyl alcohols and thermal stability for C-C Suzuki reactions.

6. Mentored new graduate students working on nanocatalysis projects.

7. Worked as Hazardous Waste Manager for two years in lab, prepared SOPs for all hazardous chemicals and Hygiene Plans for better working lab procedures.

\section{M.S. Research Project, Panjab University, Chandigarh, India, 2005-2007}

1. Synthesizing various new cobalt salts with large counter anions (1,10-phen).

2. Characterized them using various spectroscopic techniques including Elemental Analysis, FT-IR technique, NMR and X-ray Crystallography.

3. Studied the interactions of complex cations and organic anions via hydrogen bonding and how it leads to a layered structure of (001) sheets.

\section{WORK EXPERIENCE:}

Senior Research Associate at Jubilant Chemsys Limited, Noida, India, 20072010.

1. Successfully designed and executed novel synthetic routes for New Chemical Entities (NCEs) of biological importance which were screened for Structure Activity Relationship (SAR) studies for oncology drugs and in-time delivered as per client's (Eli Lilly and Company) requirement.

2. Worked on various N-heterocycles like Quinolines, Pyridothiazoles, Benzimidazoles, Pyrimidines, Imidazoles, and Pyridazines towards potent drug molecules and synthesized more than 300 finished molecules in 3 years.

3. Documented every reaction performed with chemicals, procedure, observations, characterization, results, purity and overall yield.

4. Weekly teleconferences with clients with status report.

5. Successfully managing and mentoring team of 6 training associates. 


\section{LABORATORY ANALYST:}

\section{Department of Industrial Chemistry, G.G.D.S.D. College, Panjab University -}

\section{May 2004 to April 2005}

Responsible for the preparation of stock and standard solutions for various titrations and maintaining the apparatus required for performing the experiments successfully. Responsible for maintaining the laboratory, chemicals, apparatus and equipment in an effective and ready-to-use manner. Ensured all the experiments and functions performed within the laboratory adhere to the prescribed safety standards, methods, and protocols.

\section{TEACHING EXPERIENCE:}

Graduate Teaching Assistant, Department of Chemistry, University of Louisville- January 2012 to July 2017. Taught:

1. (CHEM-207 and 208): Introduction to Analytical Chemistry I and II.

2. (CHEM-209): Introduction to Analytical Chemistry III.

3. (CHEM-343 and 344): Techniques of modern organic chemistry, mechanistic studies, identification of unknown by chemical and spectroscopic methods, special projects and synthesis and characterization in organic chemistry.

4. (CHEM-527): Principles, theoretical and experimental concepts on contemporary approaches to chemical synthesis and analysis using ${ }^{1} \mathrm{HNMR}$, HPLC, GC-MS etc.

5. (CHEM-529): Synthesis and Analysis II, synthesis and characterization of $\mathrm{Au}, \mathrm{Ag}$ nanoparticles, CdSe Quantum dots, Electro-chemistry including cyclic voltammetry, Chronoamperometry, Capillary electrophoresis etc. 
Taught students laboratory techniques, instrumentation and basic concepts of chemistry, synthesis, and characterization of various inorganic/organic chemicals. Proctored and graded course exams, lectured, supervised, and graded pre-labs and lab reports.

\section{FELLOWSHIPS, HONORS AND AWARDS:}

1. Scholarship holder, Bachelor of Science 2004-2005.

2. Outstanding Performance Award, Year 2008 and Far-Exceeding Performance Award Year 2009, Jubilant Chemsys Limited, India.

3. Departmental Teaching funding for Ph.D. research from year 2012-2017.

\section{CONFERENCE PRESENTATIONS:}

1. Bhama, S.; Zamborini, F. P. "Synthesis and thermal stability determination of various Pd nanoparticles required as an efficient catalyst for C-C cross-coupling Suzuki-Miyaura reactions." 246 ${ }^{\text {th }}$ ACS National Meeting, Indianapolis, IN, USA (2013) (Poster).

2. Bhama, S.; Zamborini, F. P. "Selective catalytic behavior of water-soluble Pd and Pd-alloy nanoparticles capped with glutathione for bilayerHydrogenation/Isomerization of allyl alcohols." $60^{\text {th }}$ SERMACS Regional Meeting, Columbia, South Carolina, USA (2016) (Poster).

3. Bhama, S.; Zamborini, F. P. "Selective catalytic behavior of water-soluble Pd and Pd-alloy nanoparticles capped with glutathione for bilayerHydrogenation/Isomerization of allyl alcohols." Graduate Student Conference, University of Louisville, KY, USA (2017) (Poster).

4. Actively participated in Science Congress Association Indian National Science academy celebrations organized on October $10^{\text {th }}, 2000$. 


\section{PUBLICATIONS:}

1. Bhama, S.; Zamborini, F. P. "Selective Catalytic Behavior of Water-Soluble Pd and Pd-Alloy Nanoparticles Capped with Glutathione for Bilayer-Hydrogenation/ Isomerization of Allyl Alcohols." (Manuscript to be submitted in Langmuir)

2. Bhama, S.; Zamborini, F. P. "Highly Active Palladium Nanoparticles for Biphasic Hydrogenation/Isomerization of Various Allyl Alcohols." (Manuscript to be submitted to the Journal of the American Chemical Society)

3. Bhama, S.; Zamborini, F. P. "Thermal Stability Determination and Catalytic Studies of Unsupported Alkyl Amine-Capped Pd Nanoparticles for SuzukiMiyaura Reaction." (Manuscript in Progress)

4. Bhama, S.; Zamborini, F. P. "Effect of Copper on Palladium for Improving the Selectivity of the Hydrogenation Reaction of Long Chain Allyl Alcohols." (Manuscript in Progress)

5. Moreno, M.; Bhama, S.; Zamborini, F. P. "Catalytic activity and Recyclability of Various Pd Nanoparticles for Hydrogenation and Isomerization of Branched Chain Allyl Alcohols." (Manuscript in Progress)

6. Harrison, W. T. A.; Slawin, A. M. Z.; Sharma, R. P.; Sharma, B.; Bhama, S. "Diaquabis(ethylenediamine)copper(II) bis(4-nitrobenzoate)." Acta Cryst. (2007) E63, m178-m180.

\section{KEY SKILLS:}

1. Highly efficient in synthesis of metal NPs with uses in catalysis and sensing applications.

2. Skill of handling milligram-scale to scale-up reactions $(1 \mathrm{mg}$ to $4 \mathrm{~kg}$ ) in laboratory including moisture-, air-sensitive reactions and hydrogenation reactions.

3. Using hazards chemicals like $\mathrm{SOCl}_{2}, \mathrm{Pd}-\mathrm{C}, \mathrm{n}-\mathrm{BuLi}$, LDA, $\mathrm{LAH}, \mathrm{NaBH}_{4}, \mathrm{MCPBA}, \mathrm{NaH}$ etc. 
4. Capable of doing collaborative and independent research work comprising multi-step synthesis in a timely manner.

5. Structural interpretation of intermediates \& finished products by using ${ }^{1} \mathrm{H}$ - and ${ }^{13}$ C-NMR, MASS, LC-MS, GC-MS, HPLC, IR etc. Material characterization by SEM, TEM, XPS, EDAX, XRD, TGA, STEM, Raman.

6. Versatile knowledge of chromatographic techniques like Biotage, Combiflash and manual gravity columns, distillation methods, crystallization and extraction methods.

7. Capable of working with eQCM and various electro-chemical techniques.

8. Knowledge of Microwave reactions in microwave reactor and Parallel Synthesizer.

9. Handling regular interactions with clients involving individual presentations.

10. Excellent communication, presentation and logic-building skills.

11. Mentoring team of training research associates on research facility expectations.

\section{PROFESSIONAL MEMBERSHIP:}

American Chemical Society (ACS), Student Member (April 2012 to Present)

\section{EXTRACURRICULAR ACTIVITIES:}

1. Attended Lab Safety and Hazardous Waste Training twice in year 2012 and 2017 as per the requirement of Department of Environmental Health and Safety (DEHS) of the University of Louisville.

2. Participated in Workshop on "English Language Usage in Business" organized by Jubilant Chemsys Ltd. on November 10th $-14^{\text {th }}, 2008$.

3. Organized Industrial Tours to the Major Industries like Mount Shivalik Breweries Limited and Saraswati Sugar mill during B.S. in the year 2003 and 2004. 


\title{
REFERENCES:
}

\author{
Dr. Francis P. Zamborini (Ph.D.) (Advisor) \\ Professor and Director of Graduate Studies \\ Department of Chemistry \\ University of Louisville, KY \\ E-mail: f.zamborini@louisville.edu
}

\section{Dr. M. Cecilia Yappert (Ph.D.)}

Professor

Department of Chemistry

University of Louisville, KY

E-mail: marta.yappert@louisville.edu

\section{Dr. Martin O'Toole (Ph.D.)}

Assistant Professor

Department of Bio-engineering

University of Louisville, KY

E-mail: martin.otoole@louisville.edu 